\title{
Assessing the cost-effectiveness of tumor necrosis factor inhibitors and prescribing practices of rheumatologists in patients with rheumatoid arthritis
}

\author{
Khalid M. Kamal \\ West Virginia University
}

Follow this and additional works at: https://researchrepository.wvu.edu/etd

\author{
Recommended Citation \\ Kamal, Khalid M., "Assessing the cost-effectiveness of tumor necrosis factor inhibitors and prescribing \\ practices of rheumatologists in patients with rheumatoid arthritis" (2005). Graduate Theses, \\ Dissertations, and Problem Reports. 4159. \\ https://researchrepository.wvu.edu/etd/4159
}

This Dissertation is protected by copyright and/or related rights. It has been brought to you by the The Research Repository @ WVU with permission from the rights-holder(s). You are free to use this Dissertation in any way that is permitted by the copyright and related rights legislation that applies to your use. For other uses you must obtain permission from the rights-holder(s) directly, unless additional rights are indicated by a Creative Commons license in the record and/ or on the work itself. This Dissertation has been accepted for inclusion in WVU Graduate Theses, Dissertations, and Problem Reports collection by an authorized administrator of The Research Repository @ WVU.

For more information, please contact researchrepository@mail.wvu.edu. 
Assessing the Cost-effectiveness of Tumor Necrosis Factor Inhibitors and Prescribing Practices of Rheumatologists in Patients with Rheumatoid Arthritis

Khalid M. Kamal

Dissertation submitted to the

School of Pharmacy

at West Virginia University

in partial fulfillment of the requirements for the degree of

Doctor of Philosophy

in

Pharmaceutical Sciences

S. Suresh Madhavan, MBA, Ph.D., Chair

Lesley-Ann Miller, Ph.D.

Virginia Scott, Ph.D.

Jan Kavookjian, MBA, Ph.D.

Jo Ann Hornsby, M.D.

Department of Pharmaceutical Systems and Policy

Morgantown, West Virginia

2005

Keywords: Rheumatoid arthritis, rheumatologist, adalimumab, etanercept, infliximab, leflunomide, methotrexate

Copyright 2005 Khalid M. Kamal 


\title{
ABSTRACT \\ Assessing the Cost-Effectiveness of Tumor Necrosis Factor Inhibitors and Prescribing Practices of Rheumatologists in Patients with Rheumatoid Arthritis
}

\author{
Khalid M. Kamal
}

Three new tumor necrosis factor (TNF) inhibitors, adalimumab, etanercept, and infliximab, have been approved for use in patients with active RA. The goals of the study were two fold: 1) to perform a cost-effectiveness analysis of the three TNF inhibitors in patients with RA that inadequately respond to MTX alone and 2) to assess the current prescribing patterns, laboratory monitoring practices, and perceived barriers of rheumatologists in prescribing these agents in patients with RA. Phase I involved the development of a Markov simulation model to estimate the health effects and costs associated with five treatment strategies in patients with RA that inadequately respond to MTX alone: (1) adalimumab plus MTX, (2) etanercept plus MTX, (3) infliximab plus MTX, (4) leflunomide plus MTX, and (5) standard therapy of MTX. A hypothetical cohort of 10,000 55-year old women was evaluated using Monte Carlo simulation. The study was conducted from a societal perspective. The main outcome measures were net gains in quality-adjusted life expectancy and incremental costeffectiveness ratios, (ICERs) in dollars per quality adjusted life year (QALY) gained. Costs and effects were discounted at 3\%. Etanercept plus MTX was the most costeffective treatment with an ICER of \$49,724/QALY. Leflunomide plus MTX was the second most cost-effective option with an ICER of \$52,833/QALY. One-way and probabilistic sensitivity analyses indicated that the conclusions were relatively stable to variations in model assumptions. In phase II, a survey was mailed to a randomly selected national sample of rheumatologists, of which $22.3 \%$ responded. The survey findings indicated that TNF inhibitor use was not restricted to moderate and severe patients with RA. Also, TNF inhibitor plus one disease modifying anti-rheumatic drug was the treatment of choice in patients with severe RA that inadequately respond to MTX alone. Costs to the patient and insurance coverage were perceived as major barriers in prescribing TNF inhibitors. One-fourth of the rheumatologists reported not using any monitoring guidelines for the TNF inhibitors indicating a need to revise monitoring guidelines or perhaps implement new guidelines for TNF inhibitors. 


\section{ACKNOWLEDGEMENT}

First and foremost, I would like to thank my advisor and Committee Chairperson Dr. Suresh Madhavan for his guidance, encouragement, and constant support throughout my dissertation and graduate studies. His patience and never wavering good nature always eased the frustration that was encountered over the years.

I would like to express my gratitude to all other committee members; Dr. Lesley Ann-Miller, Dr. Virginia Scott, Dr. Jan Kavookjian, and Dr. Jo Ann Hornsby for their invaluable suggestions and support on a variety of aspects of my dissertation research. Extra special thanks to Dr. Lesley Ann-Miller for her suggestions, assistance, and guidance in bringing my decision analysis phase to completion.

I owe a debt of gratitude to Late Dr. Anthony DiBartolomeo for some wonderful ideas that helped me initiate this research project. My sincere thanks to the entire faculty and staff at the School of Pharmacy for their constant support and encouragement. Mickey Howell, Kelly White, and Tammy Blake: Thank you for everything. The family nature of the department made me feel like I never left home.

I owe a huge thanks to my friends in the department for their help, support, and encouragement. You all made our time here in Morgantown fun and memorable. I also extend my sincere thanks to Dr. Geri Dino and the entire staff of the Prevention Research Center for their invaluable friendship and support throughout my graduate study.

The people who share the most and are a major part of this achievement are my family. My mother and father who instilled dedication, determination, hard work, and perseverance within my mindset early in my life. My brothers and sister-in-law have always given me encouragement and have been interested in my life. My wife Nazli, who agonized with me, comforted me, encouraged me, inspired and loved me. I can never thank you enough for all you have done to help me realize this dream.

Without my family I would be lost, but with you, I can do anything. 


\section{TABLE OF CONTENTS}

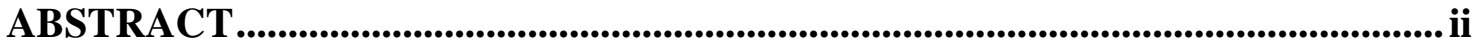

ACKNOWLEDGEMENT...........................................................................................ii

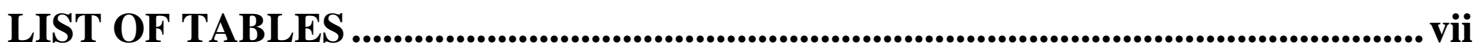

LIST OF FIGURES ............................................................................................. ix

CHAPTER ONE ……….............................................................................................. 1

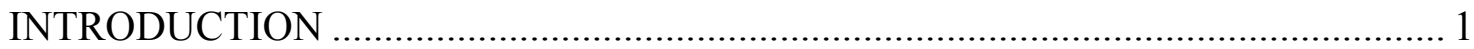

Clinical features of Rheumatoid Arthritis ............................................................. 1

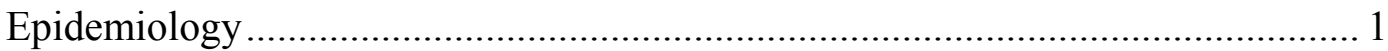

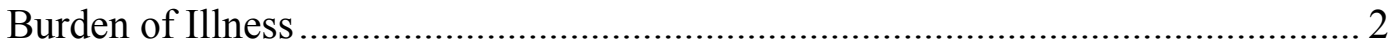

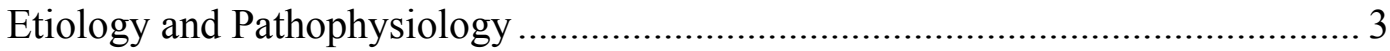

Goals of RA Management …………............................................................. 4

Assessment of Response to Treatments .............................................................

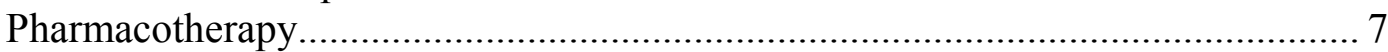

Economics of Treatments in Patients with RA that Inadequately Respond to MTX

.

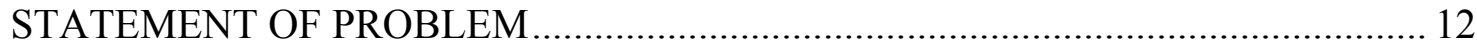

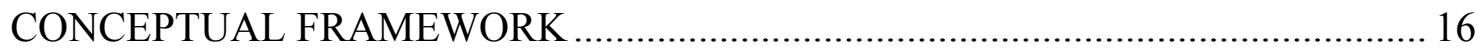

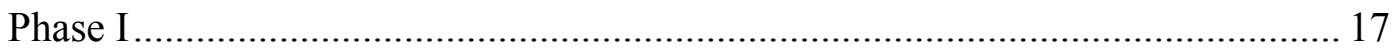

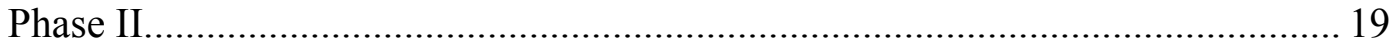

STUDY GOALS AND RESEARCH QUESTIONS …………................................. 20

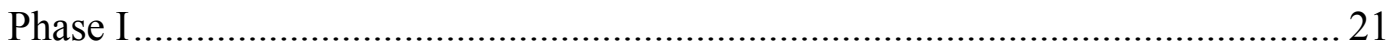

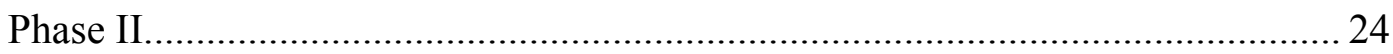

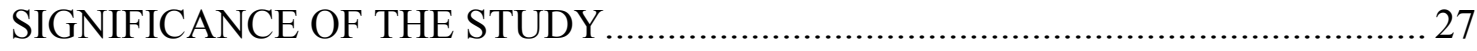

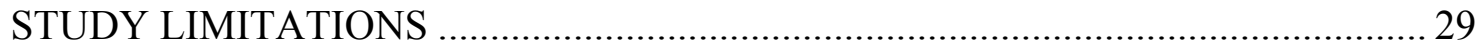

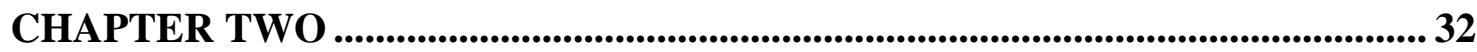

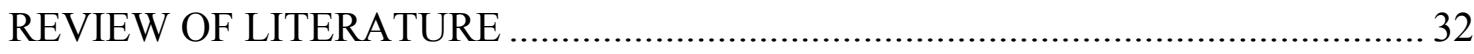

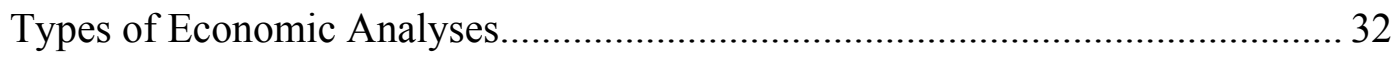

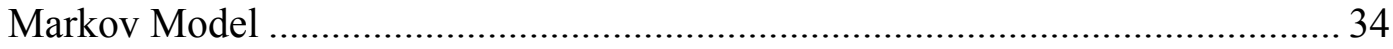

Markov models for Rheumatoid Arthritis........................................................... 39

Effectiveness of TNF Inhibitors (adalimumab, etanercept, and infliximab) and

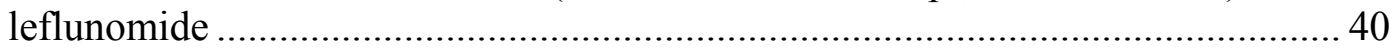

Economics of TNF Inhibitors (adalimumab, etanercept, and infliximab) and

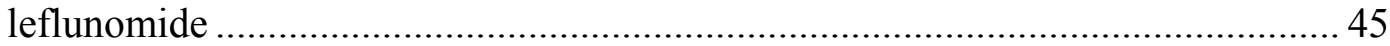

Rheumatologists' Preferences Regarding TNF Inhibitors..................................... 53

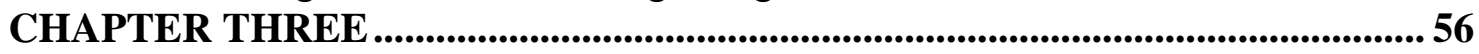

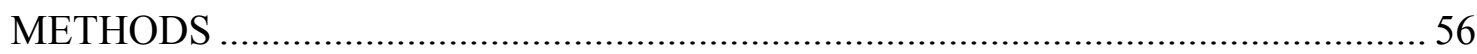

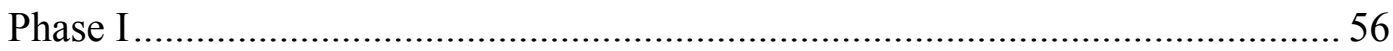

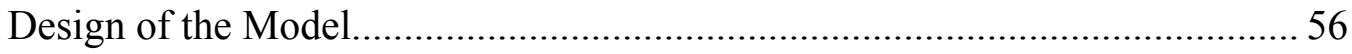

Operationalizing the Model .......................................................................... 58

Operationalizing the Model for adalimumab, etanercept, infliximab,

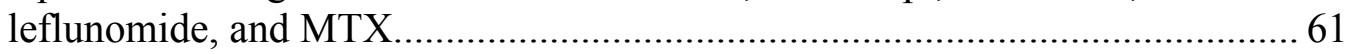

Operationalizing the Model for Joint Replacement Therapy............................... 64

Operationalizing the Model for Mortality.........................................................6 65

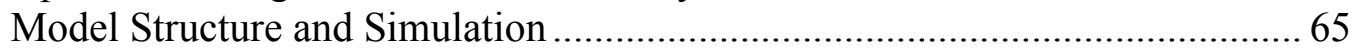


Model Assumptions and Rationale ........................................................... 70

Data for the Model ..................................................................................... 72

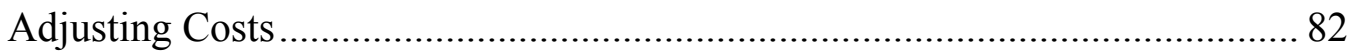

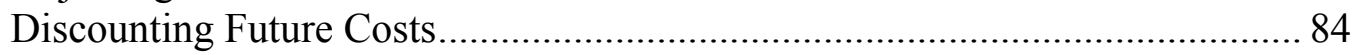

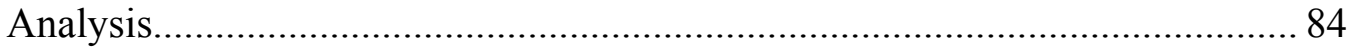

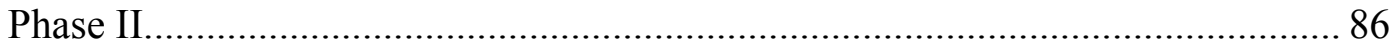

Study Population and Sample Selection ................................................... 86

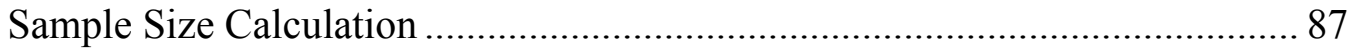

Instrument Development and Content ..................................................... 88

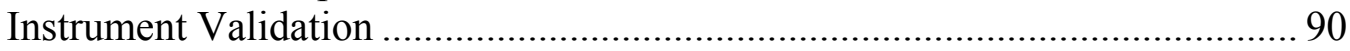

Instrument Administration ...................................................................... 90

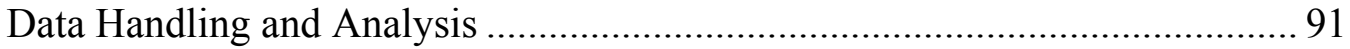

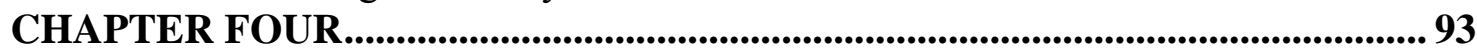

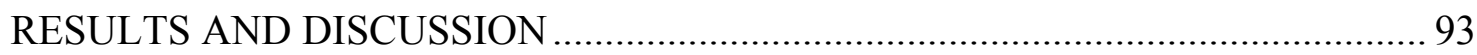

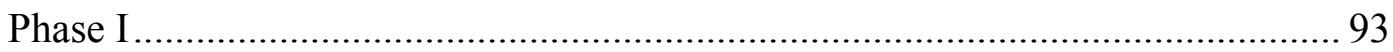

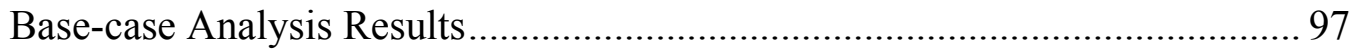

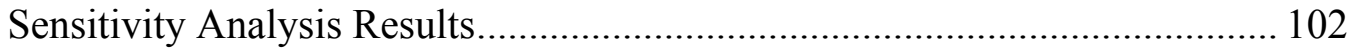

Probabilistic Sensitivity Analysis .............................................................. 125

Effect of RA-adjusted Mortality Rate on ICERs ...................................... 127

Effect of Indirect Costs on ICERs ........................................................... 130

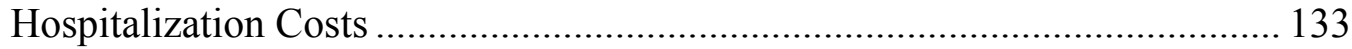

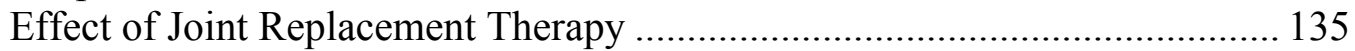

Discussion for Phase I Results.............................................................. 137

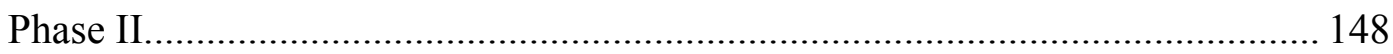

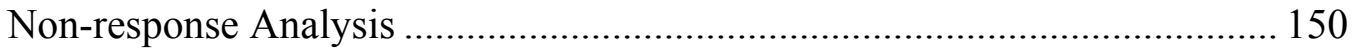

Demographics and Practice-related Characteristics of Responding

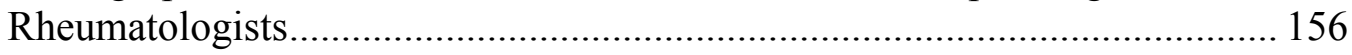

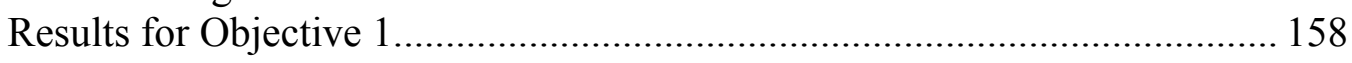

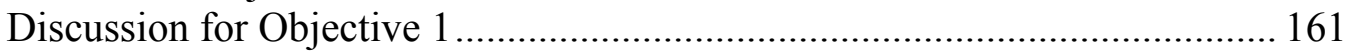

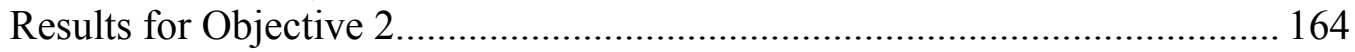

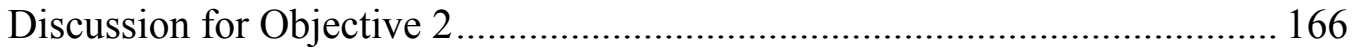

Results for Objective 3..................................................................... 167

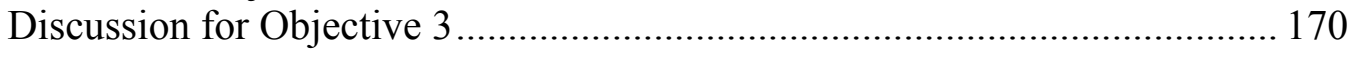

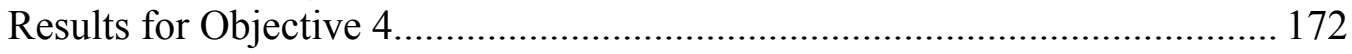

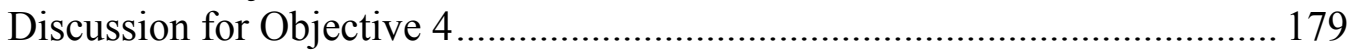

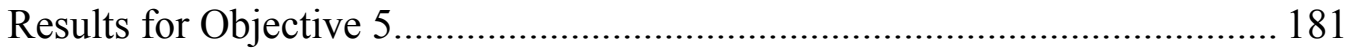

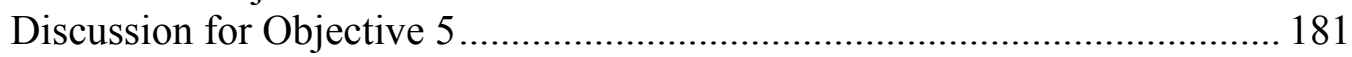

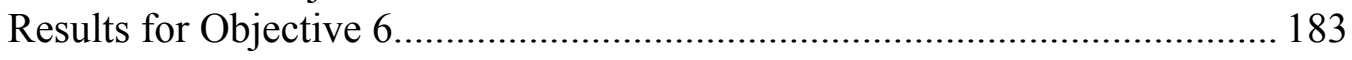

Discussion for Objective 6................................................................. 186

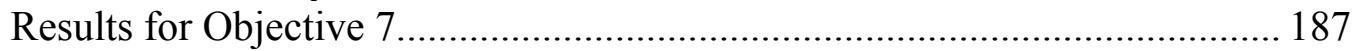

Discussion for Objective 7 ................................................................. 190

CHAPTER FIVE ............................................................................................ 192

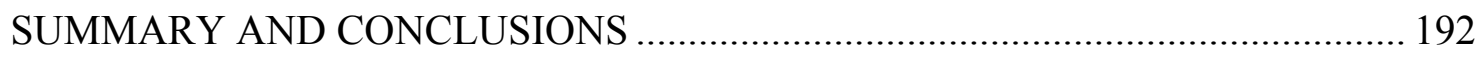

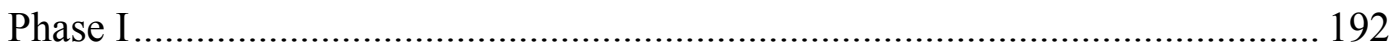

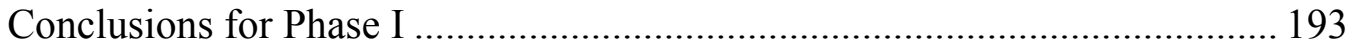

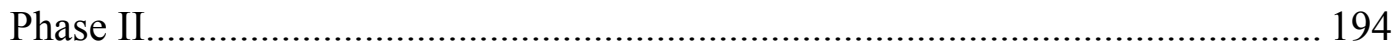




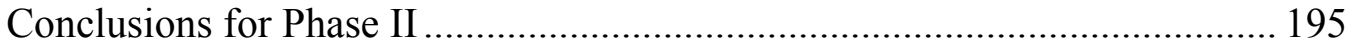

Implications of Study Findings .................................................................. 198

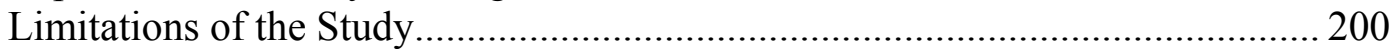

Recommendations for Future Research ....................................................... 202

BIBLIOGRAPHY ......................................................................................... 204

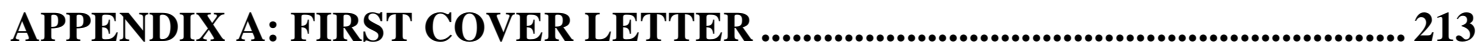

APPENDIX B: SECOND COVER LETTER ..................................................... 214

APPENDIX C: STUDY SURVEY ................................................................. 215

APPENDIX D: NON-RESPONSE SURVEY ....................................................... 220

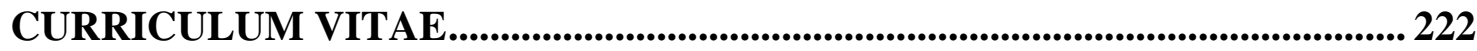




\section{LIST OF TABLES}

Table 1. A review of published RCT results for TNF inhibitors and leflunomide........ 59

Table 2. Base-case estimates (efficacy and percent probability of achieving ACR 20 response) and their ranges for sensitivity analyses: 6-month data..................... 73

Table 3. Base-case estimates (total withdrawals and subset of withdrawals) and their ranges for sensitivity analyses: 6 -month data ............................................. 75

Table 4. Base-case estimates (mild/moderate adverse event) and their ranges for

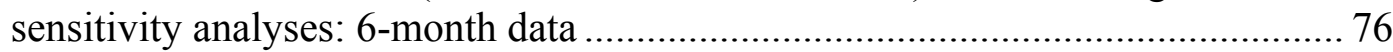

Table 5. Utilities for Markov states used in the decision analysis model .................... 77

Table 6. Costs: 6-month data used in the decision analysis model for each drug ........ 79

Table 7. Monitoring costs: 6-month data used in the decision analysis model for each drug

Table 8 . Base-case estimates and their ranges for sensitivity analyses: 6-month data.. 83

Table 9. Probability of dying based on age-, sex-, and race-adjusted all-cause mortality

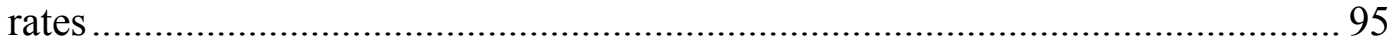

Table 10. Probability of dying based on RA-adjusted mortality rates. 96

Table 11. Base-case results for total costs and effectiveness using Monte Carlo simulations of 10,000 hypothetical patients with RA that inadequately respond to MTX (using age-, sex- race-adjusted all-cause mortality rates)....

Table 12. Base-case direct costs, total costs, effectiveness, and incremental costeffectiveness ratios of different treatments for patients with RA that inadequately respond to MTX (using age-, sex-, and race-adjusted all-cause mortality rates) 100

Table 13. One-way sensitivity analyses - Probability of withdrawals....................... 103

Table 14. One-way sensitivity analyses - Probability of withdrawals due to severe adverse events

Table 15. One-way sensitivity analyses - Probability of mild/moderate adverse event

Table 16. One-way sensitivity analyses - Probability of joint replacement and discount rate.

Table 17. One-way sensitivity analyses - Utilities: on medication, palliative care, and post-joint replacement.

Table 18. One-way sensitivity analyses - medication costs and infliximab administration costs

Table 19. One-way sensitivity analyses - Costs of monitoring in first six months ..... 109

Table 20. One-way sensitivity analyses - Costs of monitoring after six months......... 110

Table 21. One-way sensitivity analyses - Hospitalization costs ....

Table 22. One-way sensitivity analyses - Costs of treating mild/moderate adverse event, severe adverse event, joint replacement, and palliative care treatment .............. 112

Table 23. Summary of main results in one-way sensitivity analyses

Table 24. Probabilistic sensitivity analysis results for total costs, effectiveness, and incremental cost-effectiveness ratios of different treatments for patients with RA that inadequately respond to MTX (using age-, sex-, race-adjusted all-cause mortality rate 
Table 25. Results for total costs and effectiveness using Monte Carlo simulations of 10,000 hypothetical patients with RA that inadequately respond to MTX (using RA-adjusted mortality rates)

Table 26. Direct costs, effectiveness, total costs, and cost-effectiveness of different treatments for patients with RA that inadequately respond to MTX (using RAadjusted mortality rates)

Table 27. Total costs, effectiveness, and incremental cost-effectiveness ratios of different treatments for patients with RA that inadequately respond to MTX .... 131

Table 28. Total costs, effectiveness, and incremental cost-effectiveness ratios of different strategies for patients with RA that inadequately respond to MTX...... 132

Table 29. Total costs, effectiveness, and cost-effectiveness of different strategies for patients with RA that inadequately respond to MTX (without hospitalization costs)

Table 30. Proportion of patients undergoing joint replacement and age at which joint replacement occurs for each treatment ....................................................... 136

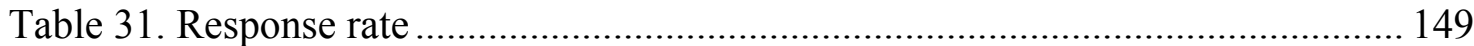

Table 32. Reasons for not participating in the study survey................................. 151

Table 33. Demographic and practice-related characteristics of respondents of the nonresponse survey.

Table 34. Patient population in which TNF inhibitors used by respondents of the nonresponse survey

Table 35. Analysis of non-response bias for demographic and practice-related characteristics.

Table 36. Demographic and practice-related characteristics of respondents.

Table 37. Respondents prescribing TNF inhibitors in patients based on the severity of the disease

Table 38. Ranking of TNF inhibitors based on rheumatologist's preference

Table 39. Demographic, practice-related characteristics, and perceived barriers of rheumatologists who preferred adalimumab, etanercept, or infliximab as their first choice TNF inhibitor. 168

Table 40. Laboratory tests ordered for adalimumab and percentage of rheumatologists ordering the tests during different weeks.

Table 41. Laboratory tests ordered for etanercept and percentage of rheumatologists ordering the tests during different weeks.

Table 42. Laboratory tests ordered for infliximab and percentage of rheumatologists ordering the tests during different weeks.

Table 43. Recommended monitoring guidelines followed by rheumatologists .......... 182

Table 44. Demographic, practice-related characteristics, and perceived barriers of rheumatologists who followed monitoring guidelines compared to those who do not follow any monitoring guidelines

Table 45. Perceived barriers to prescribing different TNF inhibitors 


\section{LIST OF FIGURES}

Figure 1. Rheumatoid arthritis treatment algorithm (ACR Guidelines) ....................... 6

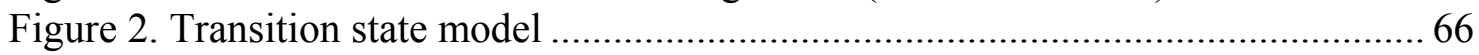

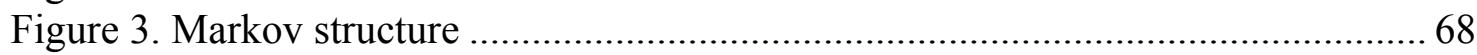

Figure 4. Total costs and effectiveness of different treatments for patients with RA that inadequately respond to MTX (using age-, sex-, race-adjusted all-cause mortality

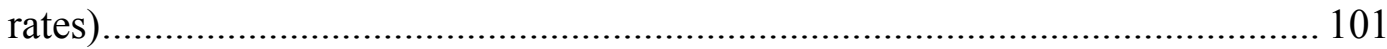

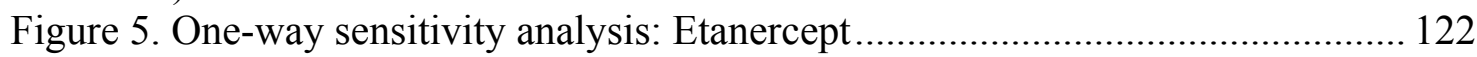

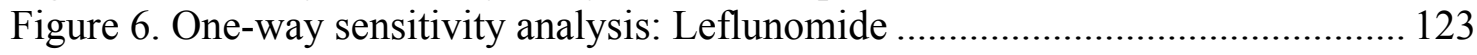

Figure 7. Perceived barriers to prescribing TNF inhibitors .................................... 188 


\section{CHAPTER ONE}

\section{INTRODUCTION}

\section{Clinical features of Rheumatoid Arthritis}

Rheumatoid arthritis (RA) is a chronic inflammatory, autoimmune disease that affects joints and other tissues. The disease is characterized by inflammation of synovial tissues, joint swelling, stiffness and pain, which may progress to joint and bone erosion. Rheumatoid arthritis generally follows one of three routes of disease progression: progressive, intermittent, or malignant. Around $70 \%$ of cases are progressive in nature, which follow a chronic pattern with periods of exacerbation and remission and significant functional limitation. Another $25 \%$ of cases are intermittent in nature and are characterized by brief attacks of inflammation with partial or complete remissions. The remaining 5\% have a malignant form of the disease with extra-articular manifestations such as vasculitis (Seymour, Worsley, Smith, \& Thomas, 2001).

\section{Epidemiology}

Rheumatoid arthritis affects around one percent of the adult population worldwide (Gabriel, 2001) and approximately 2 to 2.5 million people in the United States (ACR Guidelines, 2002; Griffiths, Bar-Din, MacLean, Sullivan, Herbert, \& Yelin, 2001). The annual incidence of RA diagnosis is about two to four people per 100,000 (Cimino \& O'Malley, 1998). Like most rheumatic disorders, the prevalence of $\mathrm{RA}$ is higher in women than men, with a reported female to male ratio of 2.5 to 1 . The 
incidence of RA is found to increase with age, with peak occurrence ranging from the fourth to sixth decade (Terebelo, 2003).

\section{Burden of Illness}

Rheumatoid arthritis is associated with pain, deformity, decreased quality of life and disability; the disease affects patients' ability to work, and hence, their socioeconomic status. In addition to causing significant morbidity and mortality, RA results in substantial medical resource use and costs. The combined direct and indirect costs resulting from RA in the United States are estimated to be approximately $\$ 26$ to \$32 billion per year (1998 values; Pugner, Scott, Holmes, \& Hieke, 2000). A recent study by Michaud and colleagues (2003) examined costs of patients with RA after the introduction of tumor necrosis factor (TNF) inhibitors. A sample of 7,527 patients with RA responded to semi-annual questionnaires from January 1999 to December 2001. Direct medical costs were calculated based on physician and other health professional visits, laboratory tests, radiological examinations, outpatient surgeries, hospitalizations, and medications. Patients on TNF inhibitors or other RA drugs reported a mean direct cost of $\$ 9,519$ (2001 values), where $66 \%$ was due to drug costs, $16 \%$ due to hospital costs, and $17 \%$ due to outpatient costs. A sub analysis of the mean direct costs revealed that the direct cost of patients on TNF inhibitors was almost three times higher $(\$ 19,016)$ than those not receiving these agents $(\$ 6,164)$.

Patients with RA have a significantly shorter life expectancy compared with the general population, with mean life expectancy shortened by seven years in males and 
three years in females (Pinals, 1987). Rheumatoid arthritis frequently leads to work disability, and the social impact of the disease is profound. Rates of work disability in the United States and Europe range from 22 to $85 \%$ and 31 to $80 \%$, respectively (Barrett, Scott, Wiles, \& Symmons, 2000). Income loss is reported to be $50 \%$ for men and $63 \%$ for women and the divorce rate in patients with RA is $70 \%$, higher than that of the general population (Allaire, Prashker, \& Meenan, 1994; Doyle, 2001).

\section{Etiology and Pathophysiology}

Despite extensive epidemiologic research, the etiology of RA is poorly understood. Rheumatoid arthritis appears to be a multi-factorial disease and a number of risk factors have been postulated in the development or progression of RA. The familial nature of this disease indicates that genetic factors are important risk factors in the etiology of this disease (Gabriel, 2001). Other suspected risk factors include infectious factors such as helicobacter pylori (Zentilin et al., 2002), Epstein Barr virus (Silman \& Pearson, 2002), and environmental factors such as cigarette smoking (Gabriel, 2001). Sex hormones are also implicated in the etiology of RA as there is an increased incidence in women (Jobanputra, Barton, Bryan, \& Burls, 2002).

Rheumatoid arthritis begins as a synovial microvasculature injury. This is accompanied by proliferation of inflammatory exudate into the joint cavity. The synovial fluid in RA is highly cellular and contains lymphocytes, macrophages, T- and B- cells. The normal joint layer consists of one to two layers of thick fibroblastic-like cells. In disease, the layer increases to a ten-cell thick layer and forms a pannus that 
actively destroys the periarticular bone and cartilage. Erosions can be seen in x-rays, which are very useful for diagnosis.

\section{Role of Tumor Necrosis Factor (TNF)}

Almost all biological processes involve cytokines. Cytokines are low molecular weight soluble proteins synthesized by different types of cells that play a key role in the function and regulation of the immune system. The most prominent cytokines include interleukins (IL-1 $\alpha$, IL-1 $\beta$ ) and TNF- $\alpha$ and $\beta$ (Toussirot $\&$ Wendling, 2004).

Interleukins and TNF play a prominent role in the mechanisms of inflammation and joint degradation as they can activate different cells in the synovium and can regulate the cartilage and bone turnover. It has been demonstrated that blocking TNF results in down regulation of pro-inflammatory cytokines such as IL-1 as well as other cytokines and angiogenic factors. Thus, TNF plays a pivotal role in the pathogenic mechanisms of RA and is a major therapeutic target in the disease treatment.

\section{Goals of RA Management}

According to the guidelines for the management of RA issued by the American College of Rheumatology (ACR), the goals of RA treatment is to prevent or control joint damage, prevent loss of function, and decrease pain (ACR Guidelines, 2002). Figure 1 depicts the RA treatment algorithm. The long-term treatment plan for RA requires a comprehensive coordinated care and the expertise of a number of health care providers. In addition, the care provided has to be reevaluated in light of clinical 
parameters and patient preferences. Clinicians incorporate various factors when choosing treatment protocols with/for their patients:

a) Therapeutic options including drug and non-drug treatments as well as their risk and benefits.

b) Modes of drug administration and monitoring for safe use of these drugs.

c) Educational needs of patients and care-givers.

d) Patient co-morbidities that may influence drug use and prognosis. For example, a number of RA patients have coexisting cardiovascular disease at diagnosis.

e) Drug costs. 
Figure 1. Rheumatoid arthritis treatment algorithm (ACR Guidelines)

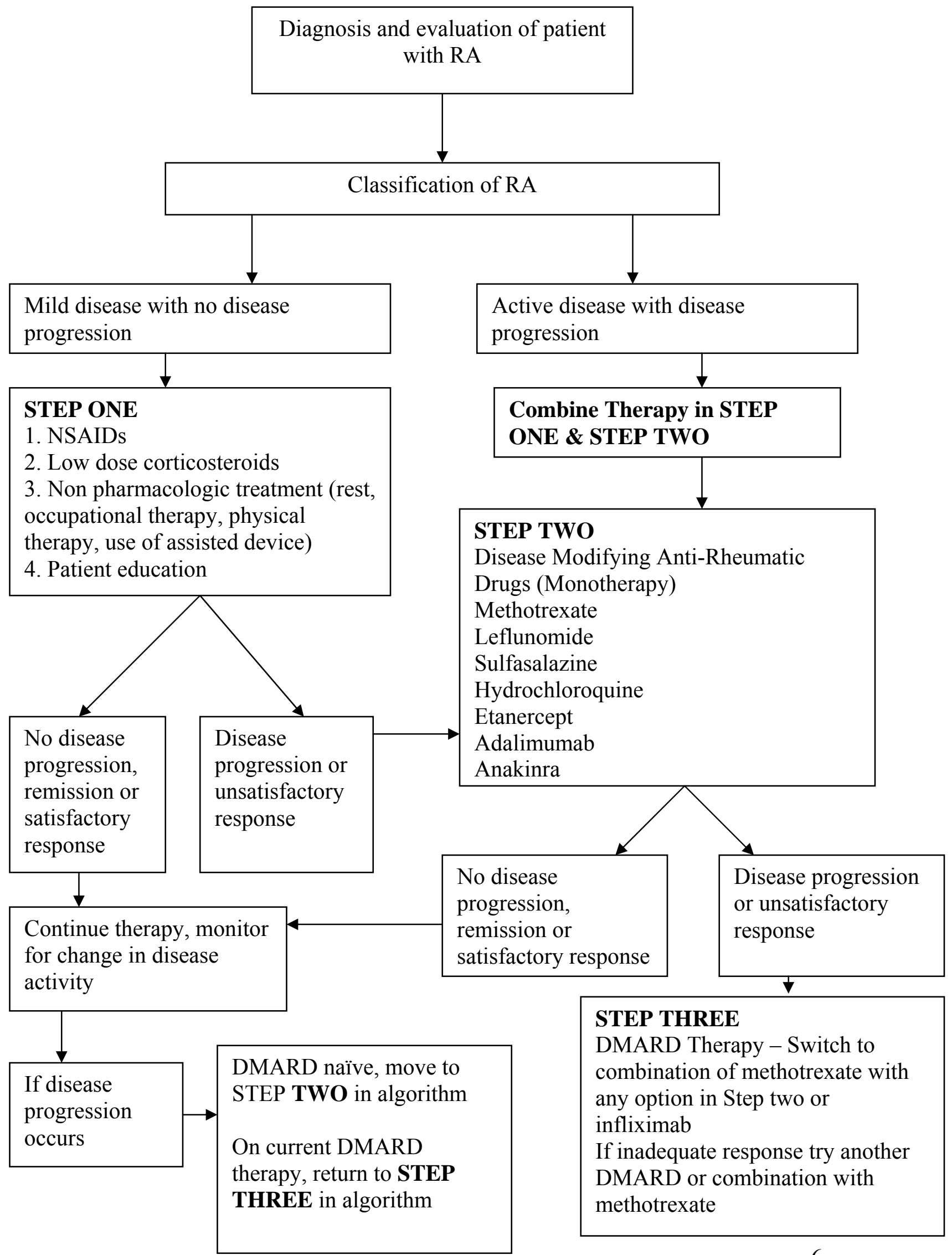




\section{Assessment of Response to Treatments}

The ultimate goals of treatment are to prevent or control joint damage, prevent loss of function, and decrease pain. In short, the goal is complete remission; this may be somewhat unrealistic in RA. The ACR has developed criteria to define improvement and clinical remission in RA. The ACR criteria of a $20 \%$ clinical response is defined as a decrease of at least $20 \%$ in the number of swollen joints, a decrease of at least $20 \%$ in the number of tender joints, and a $20 \%$ improvement in three of the following five criteria: patients' global assessment of disease status, patients' assessment of pain, health assessment questionnaire estimate of disability, physicians' global assessment of disease status, and erythrocyte sedimentation rate (ESR) or C-reactive protein (CRP) concentration. These criteria have been expanded to include criteria for a 50\% (ACR50) and a 70\% (ACR 70) improvement measures (ACR Guidelines, 2002).

\section{Pharmacotherapy}

Pharmacotherapy for active RA may include one or a combination of the following four classes of drugs: nonsteroidal anti-inflammatory drugs (NSAIDs), analgesics, corticosteroids (prednisolone and methylprednisolone), and disease modifying anti-rheumatic drugs (DMARDs) including sulfasalazine, methotrexate, gold preparations, penicillamine, azathioprine, hydroxychloroquine, leflunomide, and cyclosporin A, anakinra (interleukin inhibitor), etanercept, infliximab, and adalimumab (TNF inhibitors). 
The treatment of RA has evolved over the past few years. Four pathways of drugs for RA have been identified in the literature (Wolfe, Rehman, Lane, \& Kremer, 2001):

1) Time-based pyramid: In this approach, NSAIDs are the first line of therapy and disease modifying anti-rheumatic drugs (DMARDs) are used in the end with caution. 2) Severity-based pyramid: In this approach, the most effective treatment is given to those with more active disease.

3) Cost-based approach: The primary goal of this approach is to contain cost. Criteria are established which allow less expensive drugs to be used before more expensive ones. Additionally, use of some DMARDs and TNF inhibitors are restricted for use in patients based on severity of the disease. Thus, the severity-based approach is tied with cost considerations.

4) Patient preference: The treatment is based on the patient's needs and wishes regardless of the severity.

Studies in the literature indicate that time-based and severity-based pathways are no longer used and patients with varying severity and disease duration are treated aggressively with DMARDs and TNF inhibitors (Wolfe et al., 2001). Modern RA management stresses the importance of early diagnosis and treatment with DMARDs, particularly methotrexate (MTX) which is considered to be the "gold standard." However, several problems exist with the use of traditional DMARDs. The duration of treatment is often limited to intervals of less than two years. Patients usually discontinue the usage of these drugs either due to toxicity or lack of effectiveness. The 
next step in the management is to administer combinations of DMARDs to patients who do not respond to higher doses of single agents. Combinations include cyclosporine with MTX or hydroxychloroquine with MTX. If favorable outcomes are not achieved with these combinations, another DMARD is added to the combination, e.g. sulfasalazine with hydroxychloroquine and MTX (Kremer, 2001).

A significant number of patients (around 10\%) fail to respond to traditional DMARDs, either alone or in combination (Cairns \& Taggart, 2002). These patients appear to be resistant to conventional approaches including MTX, that is, they have used at least three DMARDs including MTX ( $\geq 15 \mathrm{mg} /$ week) and sulfasalazine (dose $\geq$ $2 \mathrm{~g} /$ day) for a minimum of six months (unless there was toxicity) and have persistent active disease despite therapy. Thus, it is apparent that further therapeutic advances are required for better treatment of the disease for those patients who do not respond to conventional approaches.

The treatment of patients with RA has changed dramatically in the last few years (Kremer, 2001; Yazici, Erkan, \& Paget, 2003). Tumor necrosis factor (TNF) has become an attractive target for treatment in patients with RA. As a result, three new TNF inhibitors, adalimumab, etanercept, and infliximab, have been approved for use in patients with RA that inadequately respond to MTX alone (Hochberg, Tracy, HoltHawkins, \& Flores, 2003). Infliximab is given in combination with MTX, while etanercept and adalimumab are administered either alone or in combination with MTX. Clinical trials of these drugs have shown that they may be more effective than 
traditional agents because of their ability to alter joint remodeling as well as attenuate symptoms (Louie, Park, \& Yoon, 2003). Leflunomide, a dihydroorotate dehydrogenase inhibitor, is another addition to the list of DMARDs, which in combination with MTX has been effective in improving signs and symptoms of patients with inadequate response to MTX (Kremer, 2001).

\section{Economics of Treatments in Patients with RA that Inadequately Respond to MTX}

A systematic literature search was conducted among literature published from January 1996 to October 2004 through MEDLINE, EMBASE, and the Cochrane library databases to identify studies that addressed the cost-effectiveness of all three TNF inhibitors and leflunomide in RA patients with inadequate response to MTX. The search yielded 14 articles of which nine were published in peer-reviewed journals and five were abstracts presented at various conferences. A study by Kavanaugh and colleagues (1996) used a six-month decision model to determine and compare the costs and effectiveness of three single agents (a hypothetical novel biologic agent, MTX, and parenteral gold. The authors reported that the total cost of MTX and parenteral gold were substantially lower than those of the hypothetical biological agent. However, sensitivity analyses indicated that by increasing the clinical efficacy of the hypothetical biological agent, total cost decreased due to the accrual of fewer indirect costs. In another study, which also employed a six-month decision model, triple therapy (a combination of hydroxychloroquine, sulfasalazine, and MTX) was the least expensive option and had the lowest incremental cost-effectiveness ratio (ICER) per ACR 20 or ACR 70 weighted response (US $\$ 1,500$ and US $\$ 3,100$, respectively). The most 
efficacious option was the combination of etanercept with MTX with ICERs of \$42,600/ACR 20 response and \$34,800/ACR 70 weighted response, respectively (Choi, Seeger, \& Kuntz, 2000). A major limitation of these studies was that early withdrawal of patients from treatments was not built into the six-month model.

Despite promising short-term results for newer TNF inhibitors, very few economic studies have addressed the long-term effectiveness of these drugs. A costeffectiveness study employing a Markov Model by Wong and colleagues (2002) demonstrated that infliximab plus MTX, when compared with MTX alone, decreased the likelihood of having advanced disability from $23 \%$ to $11 \%$ at the end of 54 weeks; this projected to a lifetime marginal cost-effectiveness ratio of $\$ 30,500$ per discounted quality-adjusted life-year (QALY) gained. A study conducted in the Netherlands, which also employed a Markov Model, reported that a treatment strategy starting with leflunomide followed by TNF inhibitors, in case of non-response with MTX, was the most cost-effective strategy (Welsing, Severens, Hartman, van Riel, \& Laan, 2004).

A UK cost-utility analysis indicated that etanercept was more cost-effective than infliximab in the treatment of patients who had failed DMARD therapy (Brennan, Bansback, Conway et al., 2001). A study by Bansback and colleagues (2004) looked at the cost-utility of adalimumab in treating patients with moderate to severe RA who have failed at least two traditional DMARDs. The cost per QALY for both adalimumab and etanercept was estimated to be between 35,000-42,000 euros 
suggesting that adalimumab was at least as cost-effective as the other TNF inhibitors.

These studies are discussed in more detail in Chapter 2.

\section{STATEMENT OF PROBLEM}

The management of patients with RA that inadequately respond to MTX alone has changed markedly with the introduction of TNF inhibitors. These agents have been shown to decrease pain, joint swelling, inflammatory arthritis, and rates of radiologic damage. Despite the benefits, these treatments are expensive and the annual cost of therapy with these drugs is approximately $\$ 16,000$ (Kavanaugh, Cohen, \& Cush, 2004).

Given the chronic nature of RA, the economic costs associated with the disease, and the high costs of the newer treatments, economic evaluations of the costs and benefits of these new agents could be very useful for optimal resource allocation. Given the current financial constraints on health care, the ability to demonstrate cost-effectiveness of new treatments is a critical factor in determining the acceptability of a new therapy (Tugwell, 2000).

One of the major concerns about economic studies in RA is the duration for which a study may be conducted. Most existing economic models determine costeffectiveness over a short period of time, since the duration of most clinical trials in RA are six months. This ignores the possibility that varying duration of efficacy in treatments can affect cost-effectiveness over a longer time horizon. Since patients with RA continue to take medications indefinitely, as long as the medication does not have any side effects, costs and outcomes continue to accumulate over a longer time horizon. 
Tumor necrosis factor inhibitors have been shown to slow down the radiological progression of the disease and thereby the downstream economic consequences. Thus, lifetime models are the most accurate way of estimating the cost-effectiveness of RA therapy. These lifetime models may also assess the impact of the treatment on the disease course, which might not be apparent in the short-term.

A few studies are reported in the literature that have conducted economic evaluations of TNF inhibitors using lifetime models (Brennan, Bansback, Reynolds, \& Conway, 2003; Kobelt, Jonsson, Lindgren, Young, \& Eberhardt, 2003; Wong et al., 2002). These studies have mainly compared one TNF inhibitor with the standard treatment (MTX) or a placebo. A major limitation of these long-term studies, however, is that none have looked at the effect of etanercept or infliximab on surgery-related hospitalizations. Disability represents a major cost-driver in RA treatment and clinical trials of these drugs have shown evidence of slowing disease progression and eventually disability (Keystone et al., 2004; Maini et al., 1999; Weinblatt et al., 2003; Weinblatt, Kremer, Bankhurst et al., 1999).

In the absence of any study evaluating the cost-effectiveness of all three TNF inhibitors in patients with RA that inadequately respond to MTX alone, there is a need to assess the impact of these agents on long-term outcomes and on disease and drugrelated morbidity and mortality. Also, it is important to determine the costeffectiveness of these new drugs in order to assess the appropriateness of their use in an increasingly cost-conscious environment. Thus, Phase I of the proposed study will 
examine the incremental cost-effectiveness of TNF inhibitors (adalimumab, etanercept, and infliximab) and leflunomide used in combination with MTX modeled over a lifetime in patients with RA that inadequately respond to MTX alone. The analysis will be conducted from a societal perspective.

Even though infliximab, etanercept, and adalimumab belong to the same class of drugs, they have distinct clinical, pharmacokinetic, and pharmacodynamic properties that must be considered when selecting any one of them for therapy. A number of factors have been found to affect a physician's decision-making process regarding choice of therapy. These include patient preference, the disease itself (for example, disease duration and symptom severity), the drug chosen (for example, potential adverse events, cost, and route of administration), and published evidence documenting the overall experience with each drug (Schwartzman, Fleischmann, \& Morgan, 2004; Schwartzman \& Morgan, 2004).

Physicians have been prescribing etanercept and infliximab for over four years. Although adalimumab has recently been introduced, the experience is greatest with the use of etanercept and infliximab. However, physicians' preference for these TNF inhibitors in patients with RA that inadequately respond to MTX have not been well studied. Another issue with these drugs is identifying the patient population that should be treated with TNF inhibitors. In their original labeling, all three TNF inhibitors were approved for patients with moderate to severely active RA. However, their actual uses have varied according to local availability, standards of practice, and monitoring 
guidelines (Kavanaugh et al., 2004). It is not known whether rheumatologists are using these agents very early in the disease course to induce remissions or for patients with active RA as indicated in the labeling of the three agents. Also, very limited data is available about the issues physicians confront in the use of these drugs or while making choices among the three TNF inhibitors. Thus, one of the goals of phase II of the proposed study is to investigate rheumatologists' current prescribing patterns for the three TNF inhibitors.

There are very few data available on these agents' possible side effects and there are no well-defined laboratory monitoring guidelines. Since the introduction of these agents, there have been concerns about the potential for adverse events as a result of inhibiting TNF. Clinical studies report that the frequency of infections and lymphomas among patients with RA treated with TNF inhibitors may be higher than in the general population. Thus, there is a need for routine screening of patients using these agents (Kavanaugh et al., 2004). However, there are currently no guidelines for blood tests to monitor patients on these agents. Studies indicate that rheumatologists using these agents seem to monitor patients based on their experience with DMARDs, particularly MTX. Also, there is no consensus as to how often the monitoring tests should be performed (Yazici et al., 2003). Hence, another goal of Phase II of the proposed study is to determine what laboratory monitoring protocols rheumatologists are using while administering TNF agents. 
The selection of the most appropriate agent in RA treatment is a complex clinical decision. The TNF inhibitors have been shown to achieve a marked improvement in clinical outcomes in patients with RA. However, there are a number of concerns regarding their use by rheumatologists. These include the potential for adverse events (such as injection and infusion-site reactions, upper respiratory tract infections), severe infections, high cost of TNF inhibitors, route of administration, and patient preference (Fleischmann \& Yocum, 2004). Thus, one of the goals of Phase II of the proposed study is to investigate the different factors that rheumatologists perceive as problematic in prescribing specific TNF inhibitors for patients with RA.

In summary, Phase I of the proposed study will examine the incremental costeffectiveness of the three TNF inhibitors and leflunomide in combination with MTX in patients with RA that inadequately respond to MTX alone. In Phase II of the study, rheumatologists' current prescribing patterns, laboratory monitoring protocols, and perceived barriers to prescribing the three TNF inhibitors will be assessed.

\section{CONCEPTUAL FRAMEWORK}

This study will be conducted in two phases. Phase I will involve the construction of an economic model to compare the incremental cost-effectiveness of the three TNF inhibitors and leflunomide in combination with MTX in patients with RA that inadequately respond to MTX alone. Phase II will assess the current prescribing patterns of rheumatologists using TNF inhibitors in treating patients with 
RA. Information on laboratory monitoring practices, frequency of tests ordered, and perceived barriers of rheumatologists to prescribing these drugs will also be assessed.

\section{Phase I}

Phase I involves developing a decision analytic model to compare the total costs and effects of each of the three TNF inhibitors (etanercept, infliximab, and adalimumab), and leflunomide used in combination with MTX in patients with RA that inadequately respond to MTX alone. Economic analysis utilizing decision analytic models is a comparative analysis of different therapeutic options in terms of their costs, efficacy, and safety parameters. The end result of this analysis is to summarize the benefits and harms (both clinical and financial) of the different therapeutic options and identify those options that deliver maximum benefits in the most cost efficient manner (Homik \& Suarez-Alamor, 2004).

The decision analytic model in the proposed study will assume that a base-case population of 55 year-old women have already failed at least two DMARDs, one of which is MTX. The selection of the base-case population is because it is the patient population that has been studied in the majority of the randomized clinical trials (RCTs), and is therefore most representative of the patients with RA receiving TNF inhibitors and leflunomide. Since it is assumed that the patients will inadequately respond to MTX, the five treatment options to be considered in the analysis will include combination of each of the three TNF inhibitors (adalimumab, etanercept, infliximab) with MTX, leflunomide plus MTX and standard therapy of MTX. 
The decision model to be employed in the analysis will be a Markov Model with six-month cycles. Since the current study involves extrapolating results from short-term RCTs over a patient's lifetime to quantify the effects of introducing the treatments, Markov models are particularly suited to handle both costs and outcomes simultaneously over chronic time intervals (Briggs \& Sculpher, 1998; Sonnenberg \& Beck, 1993). Details on the actual working of Markov models are presented in Chapter 2. In each six-month cycle of the model, the patient will either continue with the treatment or will withdraw due to severe adverse events or lack of efficacy. In the event of withdrawal, the patients will be switched to palliative care where they will be treated with a combination of MTX, NSAIDs, and corticosteroids. The effectiveness measures will consist of American College of Rheumatology 20\% response criteria (ACR 20). Those who will continue with the treatment may respond to treatment by at least 20\% (ACR 20) or may experience adverse events (mild and moderate) minor enough to continue with the treatment. Thus, at the end of each cycle, patients will be redistributed in the model based on the transition rates derived from the RCTs of the respective treatments. The model will run until all the patients are dead.

Rheumatoid arthritis, like other chronic diseases, has a continuous effect on several functions over a long period of time and therefore several outcome measures are used. However, RA-specific outcomes like ACR 20 cannot be used to make absolute statements about whether a given option is cost-effective compared with other widely accepted cost-effective interventions in medicine (Choi et al., 2000). Thus, a generic measure of effectiveness such as quality-adjusted life years (QALYs) will also 
be used in the study. Quality-adjusted life years combines QoL with time by adjusting life-years with a quality weight, measured as utility. Utilities, to calculate QALYs in the present study, will be derived from a study by Bansback and colleagues (2004).

A societal perspective will be taken in the estimation of costs in the base-case analysis. The total cost of treatment with each agent is composed of direct costs associated with treating patients with RA that inadequately respond to MTX, combined with the indirect costs (for example, loss of productivity and premature mortality) incurred by the patient as a result of the disease. Incremental cost-effectiveness ratios will be calculated as additional cost per patient achieving outcomes, compared with the next more expensive option.

\section{Phase II}

As noted earlier, treatments for RA have evolved over time. Randomized clinical trials are considered as the "gold standard" for assessing the value of a therapy. However, the short-term data derived from RCTs may not be applicable to long-term effectiveness of controlling the disease, especially in a chronic and complex disease like RA. Long-term observational studies are also used to evaluate therapies but these require investment in terms of time and money. A third approach, in addition to RCTs and long-term observation studies, is to survey rheumatologists regarding their opinions on newer therapies (Wolfe, Albert, \& Pincus, 1998). 
Since the TNF inhibitors have recently been introduced in the market, there is not enough data available regarding their safety and effectiveness. Also, there are no recommended treatment guidelines available for rheumatologists to consult, while using these drugs. The selection of etanercept, infliximab, or adalimumab depends on a number of factors. They have different routes of administration, which can affect a patient's preference for the drugs. Safety (severe adverse events, moderate events, and mild events like injection- and infusion-related reactions) is another factor which physicians may consider to make their choice. In terms of reimbursement issues, intravenous (IV) dosing (infliximab) may hold an advantage over subcutaneous (SC) dosing (etanercept and adalimumab), especially in patients with Medicare coverage. This information can only be clarified with the availability of more data from a clinical trial or from evaluation of physician practice experiences.

Phase II of the proposed study will assess the current prescribing patterns of rheumatologists using TNF inhibitors in treating patients with RA through a national mail survey. The survey will be useful in providing data on the clinical experiences of rheumatologists. In addition, information on laboratory monitoring practices, frequency of tests ordered, and perceived barriers of rheumatologists in prescribing TNF inhibitors will also be collected.

\section{STUDY GOALS AND RESEARCH QUESTIONS}

The goals of the study are two fold: 1) to conduct an incremental costeffectiveness analysis of TNF inhibitors (adalimumab, etanercept, and infliximab) and 
leflunomide used in combination with MTX in the treatment of patients with RA that inadequately respond to MTX alone using a Markov simulation model, and 2) to assess the current prescribing patterns, laboratory monitoring practices, and perceived barriers of rheumatologists in prescribing TNF inhibitors in patients with RA

\section{Phase I}

Phase I will involve the development of a Markov simulation model to calculate the incremental cost-effectiveness ratios of TNF inhibitors (adalimumab, etanercept, and infliximab) and leflunomide used in combination with MTX in the treatment of patients with RA that inadequately respond to MTX alone

\section{Objectives for Phase I:}

Objective 1: To develop a Markov simulation model to assess the long-term costs and consequences of TNF inhibitors (adalimumab, etanercept, and infliximab) and leflunomide used in combination with MTX in the treatment of patients with RA that inadequately respond to MTX alone

Rationale: Decision analysis modeling is a powerful simulation technique and is being increasingly used to assess the economic outcomes of new drugs. Decision analysis modeling can be an inexpensive and effective way of synthesizing existing data and evidence available on costs and outcomes of alternative treatments, and can be used to extrapolate beyond the follow-up period of a clinical trial, or from an intermediate outcome to a final health outcome (Brennan \& Akehurst, 2000). These analytic models can assist decision makers until more definitive information is made available about 
outcomes and costs in actual clinical settings. Rheumatoid arthritis is a chronic, debilitating, and lifelong disease and any decision analytic model developed to evaluate the cost-effectiveness of the new drugs needs to take into account the lifetime costs of the therapies. Decision tree models are not appropriate since they report the outcomes over one to two-year time frame. Also, there is a limit to the manageable size of the decision tree. Markov models overcome this hurdle and provide a better way of simulating the progression of RA over time and the possible impact of treatments.

Objective 2: To develop cost-effectiveness models based on the Markov simulation model that determine the incremental costs and consequences of TNF inhibitors (adalimumab, etanercept, and infliximab) and leflunomide used in combination with MTX in the treatment of patients with RA that inadequately respond to MTX alone Rationale: When considering a drug therapy for RA, several factors appear to be predictive of positive cost-effectiveness. Disability is a major determinant of expense in RA and any drug that slows joint destruction and thus delays the onset of disability has the potential to be cost-effective. Another determinant of RA expense is the cost associated with treating drug-related adverse events. Thus, a drug with good safety profile will probably be more cost-effective than a drug with similar efficacy but causing more side effects. Finally, RA being a chronic disease, the longer the drug remains effective and tolerable, the more likely it will be cost-effective (Tugwell, 2000). 
The introduction of TNF inhibitors (adalimumab, etanercept, and infliximab) represents a significant advance in the available treatments for patients with RA. These agents have demonstrated good efficacy and tolerability in clinical trials (Keystone et. al., 2004; Kremer et. al., 2002; Maini et. al., 1999; Weinblatt et al., 2003; Weinblatt et. al., 1999). These agents have also been shown to be more effective than traditional agents because of their ability to slow disease progression and to improve disability scores. While all these attributes suggest that these agents will be cost-effective, only appropriate analyses that examine both efficacy and total costs can provide a true assessment of cost-effectiveness (Tugwell, 2000).

A few studies have been conducted to evaluate the cost-effectiveness of these agents. A study by Choi and colleagues (2000) that used a six-month decision model failed to account for early withdrawals by patients who did not respond to the treatments. Similarly, two studies that used long-term models, let non-responders stay on treatment for one full year (Kobelt et al., 2003; Wong et al., 2002). Neglecting to incorporate early withdrawals potentially biases the results because patients that fail to respond to treatment will show little gain in health improvement but will continue to accrue large costs associated with one-year treatment with TNF inhibitors. In addition, most of the studies failed to include the decrease in future risk of disability, which could improve the cost-effectiveness of treatments. Conversely, the adverse effects of TNF inhibitors (including high risk of serious infections), which may increase health care costs, were not included in most analyses. Simply demonstrating the efficacy of a treatment does not necessarily establish its economic benefits. Economic evaluation of 
the TNF inhibitors should include their ability to prevent disability and safety profiles, and should be conducted over a patient's lifetime thereby determining the true costeffectiveness of these agents.

\section{Phase II}

The primary goal of phase II will be to assess rheumatologists' current prescribing patterns for the three TNF inhibitors. Secondary goals will include determining rheumatologists' laboratory monitoring practices, practice of switching patient from one TNF inhibitor to another TNF inhibitor, and identifying perceived barriers to prescribing these drugs.

The specific objectives are as follows:

\section{Objectives for Phase II:}

Objective 1: To describe rheumatologists' prescribing patterns for the three TNF inhibitors: adalimumab, etanercept, and infliximab.

Rationale: Recent surveys have shown that combinations of DMARDs are preferred in practice especially to treat patients with moderate or severe RA (Jobanputra, Wilson, Douglas, \& Burls, 2004; Maetzel, Bombardier, Strand, Tugwell, \& Wells, 1998; Pope, Hong, \& Koehler, 2002). With the introduction of new TNF inhibitors, it is important to investigate the current prescribing patterns of rheumatologists. The labeling of these drugs indicates their use in moderate to severe patients. But whether these drugs are being prescribed as first-line therapy in RA alone or used in conjunction with other traditional DMARDs is not known. 
An indirect comparison of the three TNF inhibitors by Hochberg and colleagues (2003) indicated similar efficacies in RA. Thus, a reasonable question for clinicians is whether patients who have failed one TNF inhibitor could be given a trial with another TNF inhibitor. A few studies conducted in rheumatology clinics have reported switching patients from one TNF inhibitor to another TNF inhibitor, but it is not known if rheumatologists in different practice settings are doing the same and their reasons for switching patients from one TNF inhibitor to another TNF inhibitor (Haraoui, 2004; Yazici \& Erkan, 2004).

Objective 2: To determine if the rheumatologists have any preference for a particular TNF inhibitor.

Null Hypothesis A: Rheumatologists do not have any preference for any of the TNF inhibitors.

Objective 3: To assess if there are any differences in demographic (age, gender) and practice-related characteristics (number of years in practice as a rheumatologist, primary practice site, average number of RA patients seen per week) and perceived barriers in prescribing TNF inhibitors among rheumatologists who prefer adalimumab, etanercept, or infliximab as their first choice agent.

Null Hypothesis B: There are no significant differences in demographic (age, gender), practice-related characteristics (number of years in practice as a rheumatologist, primary practice site, average number of RA patients seen per week), and perceived 
barriers in prescribing TNF inhibitors among rheumatologists who prefer adalimumab, etanercept, or infliximab as their first choice agent.

Objective 4: To describe the laboratory monitoring practices and the frequency of tests ordered by the rheumatologists for patients on TNF inhibitors.

Rationale: There are no known monitoring guidelines established for TNF inhibitors. There are a number of reports on the safety profile of these agents and at the same time a dearth of information regarding their long-term outcomes. According to the only study conducted to find whether or not rheumatologists follow monitoring guidelines, results indicated that rheumatologists seem to continue their practice of monitoring based on their experience with earlier traditional DMARDs. However, there seems to be a significant variation in laboratory tests ordered, and the frequency of ordering these tests (Yazici et al., 2003). This research question is a follow-up on this study to determine if the practice is the same or has changed over the years.

Objective 5: To determine if the rheumatologists are following any recommended monitoring guidelines for patients with RA on adalimumab, etanercept, and infliximab. Null Hypothesis C: Rheumatologists are not following any recommended monitoring guidelines for patients with RA on adalimumab, etanercept, and infliximab.

Objective 6: To assess if there are any differences in demographic (age, gender) and practice-related characteristics (number of years in practice as a rheumatologist, primary practice site, average number of RA patients seen per week) and perceived 
barriers in prescribing TNF inhibitors between rheumatologists who follow monitoring guidelines and those who do not follow any guidelines.

Null Hypothesis D: There are no significant differences in demographic (age, gender) and practice-related characteristics (number of years in practice as a rheumatologist, primary practice site, average number of RA patients seen per week) and perceived barriers in prescribing TNF inhibitors between rheumatologist's who follow guidelines and those who do not follow any guidelines.

Objective 7: To assess if there are any differences in rheumatologists' perceptions of barriers (insurance coverage, route of administration, patient compliance, patient preference, side effects, costs to the patient, age of the patient, efficacy of the drugs, and support needed for administration of drugs) to prescribing the three TNF inhibitors. Null Hypothesis E: There are no significant differences in rheumatologists' perceptions of barriers (insurance coverage, route of administration, patient compliance, side effects, costs to the patient, age of the patient, efficacy of the drugs, and support needed for administration of drugs) to prescribing the three TNF inhibitors.

\section{SIGNIFICANCE OF THE STUDY}

Recent therapeutic advances in the treatment of RA have demonstrated improvement in inflammatory arthritis and stabilization of radiographic progression, but these new therapies are expensive due to high acquisition costs, potential toxicity, and associated monitoring. The costs of not treating RA are also very high due to increased medical and long-term costs associated with uncontrolled disease. The cost- 
effectiveness of TNF inhibitors is presently unclear. The results of Phase I of the study can help determine if etanercept, infliximab, adalimumab, or leflunomide used in combination with MTX in patients with RA that inadequately respond to MTX alone is cost-effective or not. The TNF inhibitors have been shown to prevent and delay disability. At the same time, they have also been shown to cause serious adverse events. The results of the study may also determine if the reductions in nondrug costs can help offset the high acquisition costs of these agents and the high health care costs due to higher risk of infections.

However, as discussed earlier, the choice of an optimal agent by a rheumatologist is based not only on cost but also on accumulated clinical experience, patient preference, convenience, route of administration, and safety profile of a drug. The most widely accepted and used guidelines are for MTX and published by the ACR. For the newer TNF inhibitors, risks exist and infections, neoplasms, and autoimmune disorders have been reported in patients using these agents. It is important that drug toxicity is discovered as early as possible by appropriate laboratory monitoring before serious events become clinically evident. Currently, very little information exists with regards to the rheumatologists' prescribing patterns for TNF inhibitors, laboratory monitoring protocols, and their perceived barriers in prescribing these agents. Consequently, Phase II of the study was developed to study rheumatologists' use of the TNF inhibitors. Results of phase II study will help provide valuable insight into the prescribing patterns of rheumatologists using these agents for treatment of patients with RA. 


\section{STUDY LIMITATIONS}

The limitations of each phase of the study are discussed below:

The main limitation of Phase I of the study relates to the data available. An ideal source would have been a very long-term, randomized study with a large sample size examining the efficacy and resources utilized by all the therapeutic options in RA patients who have inadequate response to MTX. In the absence of such a study, evidence has to be assembled from a range of sources and these sources have their advantages and disadvantages from a health economic perspective. Data from clinical trials do not have high external validity. In addition, data on actual use of etanercept, infliximab, and adalimumab are not yet available. Thus, the results of any modeling study need to be treated with some degree of caution.

Another limitation in Phase I is that the base-case analysis in the decision analytic model assumes that the baseline characteristics of the study patients across the source RCTs are comparable based on their close similarities in important baseline demographic and clinical characteristics. It is a general view that absolute comparability across different treatments can be established through randomization. Currently, such data does not exist and it appears doubtful that all the treatment options considered in the analysis would be evaluated using a single head-to-head randomized controlled clinical trial in the near future. 
The efficacy data for each treatment option (except for adalimumab plus MTX) comes from a single respective RCT. Therefore, there will be generalizability issues with the base-case estimate used in the Phase I of the study. However, sensitivity analyses will be conducted to address this limitation.

As discussed in the introduction, the significant portion of health services costs incurred by RA patients is due to surgery-related hospital admissions. It is possible that effective disease control with more effective therapy could prevent or reduce hospital admissions. A study by Yelin and Wanke (1999) reported that almost 52\% of the direct costs of RA was due to hospital admissions, of which $95.2 \%$ came from surgical admissions. The authors also reported that functional status measured by Health Assessment Questionnaire (HAQ) disability score was the only consistent and strong predictor for the total direct costs. The surgery-related costs for each treatment strategy was calculated based on an exponential relationship between HAQ score reported from clinical trials and inpatient surgery costs used by Choi and colleagues (2000). However, since the data may not be reliable, the study will report results both by including and excluding surgery-related costs in the model.

The three TNF inhibitors and leflunomide have different modes of administration. However, the potential impact of compliance was not considered in the model. No data is available indicating differing rates of compliance among the different TNF inhibitors and leflunomide in patients with RA. 
In RA, indirect costs can be divided into morbidity costs (lost production) and mortality costs (present value of lost production due to premature death). In the present study, indirect cost was estimated as being one to three fold higher than direct costs, which may limit its accuracy.

Phase II of the study will use a mail survey to elicit current treatment practices of rheumatologists. The study therefore will have all the limitations associated with cross-sectional survey design methodology.

This chapter gave a brief description of underlying health problem, the need for the study, the research questions, the significance and the limitations of the study. The next chapter will give an extensive review of the existing literature associated with RA and TNF inhibitors. 


\section{CHAPTER TWO}

\section{REVIEW OF LITERATURE}

The first section of this chapter will describe the techniques of economic evaluation and will provide an overview of the use of Markov models in performing these evaluations. A review of the clinical effectiveness and available pharmacoeconomic evaluations of tumor necrosis factor inhibitors and leflunomide in RA patients that inadequately respond to MTX alone will be discussed next. Finally, studies investigating rheumatologists' prescribing patterns for specific TNF inhibitors and their use of monitoring protocols will be reviewed.

\section{$\underline{\text { Types of Economic Analyses }}$}

Rheumatoid arthritis (RA) is a chronic systemic disorder and is associated with pain, functional limitations, physical disability, and decreased QoL (Blumenauer, Coyle, \& Tugwell, 2002). As a chronic debilitating disease with a progressive course, RA has an economic impact that is disproportional to its prevalence. Several studies have been conducted to quantify the economic costs of interventions that delay or prevent disability and reduce the associated morbidity and mortality in RA. However, these studies need to include both costs and the consequences to establish the economic benefit of the therapy.

Studies that consider both costs and consequences are termed as "full' economic evaluation and include cost-benefit analyses, cost-effectiveness analyses, and cost- 
utility analyses. These studies consider the total direct, indirect, and intangible costs. Direct costs include physician services, hospitalizations, medication costs, diagnostic procedures (laboratory and radiological costs), costs of treating of adverse events, patient and caregiver time, and rehabilitation and nursing care. Indirect costs include loss of income due to loss of workdays or loss of employment, house-remodeling costs, and the intangible costs of impact on social life, pain, deformity, and decreased QoL (Blumenauer et al., 2002; Drummond, O’Brien, Stoddart, \& Torrance, 1998; Emery, 2004; Homik \& Suarez-Almazor, 2004).

Cost-benefit analyses express both costs and consequences in monetary terms. These methods are less commonly used, as they require the consequences of the treatment to be expressed in dollar terms. The most commonly used economic evaluations in RA are cost-effectiveness and cost-utility analyses. Cost-effectiveness analyses measure costs in terms of a defined measure of a clinical outcome; cost-utility analyses measure costs in relation to an outcome, and take QoL into consideration (e.g. Quality adjusted life years or QALYs). These types of analyses are more appropriate since they not only include the total costs of the therapy but also the costs of the disease process. Patients with active RA may have major disabilities resulting in loss of workdays, decreased QoL, and other outcomes; cost-effectiveness analyses account for such non-health expenditures (Drummond et al., 1998; Emery, 2004; Homik \& SuarezAlmazor, 2004). 
As opposed to full economic evaluations, "partial" economic evaluations consider only the cost of intervention (direct and indirect) and not the outcome (clinical effectiveness or economic benefit) of the intervention (Drummond et al., 1998; Emery, 2004; Homik \& Suarez-Almazor, 2004). Cost analyses are considered as partial economic evaluations and are useful in evaluating the impact of drugs with similar efficacies. However, cost analyses of the TNF inhibitors against the traditional DMARDs might not favor the TNF inhibitors due to their high acquisition costs (Lipsky \& Kavanaugh, 1999). A full economic evaluation of TNF inhibitors and traditional DMARDs, on the other hand, may indicate TNF inhibitors to be costeffective since these evaluations take into account the downstream economic savings (e.g., surgery-related hospitalizations) that might offset the high acquisition costs of TNF inhibitors. Thus, cost-effectiveness and cost-utility studies are the most appropriate studies in evaluating the impact of TNF inhibitors in RA.

\section{Markov Model}

One key issue when performing cost-effectiveness analyses of new treatments in chronic conditions like RA is that the randomized clinical trials to date are for short time periods compared to the lifetime duration of the disease. Also, clinical trials exhibit modest external validity due to exclusion of certain age groups or comorbidities and protocol driven costs (Brennan \& Akehurst, 2000). Observation studies may provide useful data. However, there is limited data available on the use of new treatments in clinical practice (Moots, Taggart, \& Walker, 2003). 
One approach in performing economic analyses is to apply modeling techniques to available data. Economic modeling can be an inexpensive and effective way of synthesizing existing data and evidence available on costs and outcomes of alternative treatments, and can be used to extrapolate beyond the follow-up of a trial or from an intermediate to a final health outcome (Brennan \& Akehurst, 2000). A number of decision models such as decision trees and Markov models have been reported in the literature for conducting cost-effectiveness analyses of TNF inhibitors. The following is a brief description providing a rationale for choosing a Markov model over a decision tree in the proposed study.

The decision tree is the simplest structure used to represent possible patient treatment pathways. These pathways can be modeled using probabilities of events and relevant outcome measures. Decision trees are usually employed when the time frame of the model is short and there is no difference in mortality across treatment strategies. This, however, ignores the possibility that varying duration of efficacy in treatments can affect cost-effectiveness over a longer time horizon. Rheumatoid arthritis is a chronic, debilitating, and lifelong disease and patients with RA continue to take medications indefinitely as long as the medication does not have any side effects. Thus, costs and outcomes continue to accumulate over a long time horizon and any decision model developed to evaluate the cost-effectiveness of the new drugs need to take into account the lifetime costs of the therapies (Emery, 2004). If a decision tree model is used to report the outcomes over a very long period of time, the tree structure may become unmanageable; with the presence of repetitive outcomes, the structure of 
the decision tree can get complicated. A Markov model overcomes this hurdle by allowing incorporation of time-dependent events.

Markov models are used in decision analysis to represent stochastic processes, which evolve over time (Briggs \& Sculpher, 1998; Sonnenberg \& Beck, 1993). As a result, they are particularly suited to modeling chronic diseases. The disease in question is divided into a finite number of health states usually defined by the severity of the disease. The individual is assumed to be in any one of the health states referred to as Markov states. All events are then considered as transitions of individuals from one health state to another. The time horizon in Markov models is divided into equal increments of time, referred to as Markov cycles, and the length of the cycles are chosen to represent a clinically meaningful time interval.

During each cycle of the model, transitions are assumed to take place and the net probability of making a transition from one state to another during one cycle is called a transition probability. It is assumed that a patient in a given state can make only a single state transition during a cycle. A patient may remain in one state for a number of cycles or may transition to a different state. There are states in the Markov model from which it is impossible for patients to leave; these are called absorbing states. The most common example of an absorbing state is death.

An important limitation of the Markov model is that the probability of moving out of the state is not dependent on the state or states a patient may have experienced 
before entering the current state. In other words, a Markov state does not depend on history. This is often referred to as the 'Markovian assumption' and it can be accounted for by using a combination of distinct states or time-dependent transition probabilities (Briggs \& Sculpher, 1998; Sonnenberg \& Beck, 1993).

Markov models are categorized into two different types of models based on whether the state transition probabilities are constant over time or not. Markov chains are models in which the transition probabilities are constant over time. Markov models where transition probabilities vary with time are called time-dependent Markov processes and are more flexible in modeling a chronic disease.

In order to complete a Markov model, it is important to attach weights to the model for costs and consequences to be estimated. For example, the calculation of QALYs or costs over the lifetime of the model will involve weighting the length of time spent in a particular health state by a value representing either QoL experienced in that state or the costs incurred in that state. A QALY is an effectiveness measure that includes both quantity and QoL, by assigning a "utility" weight to life-years.

In economic analyses, utility refers to preferences which individuals or society place on particular health outcomes and is measured on a scale between zero (dead) and one (perfect health). Utility can be elicited directly by using techniques such as standard gamble, time trade-off, and rating scale or can be elicited indirectly by using questionnaires such as EuroQol (EQ-5D) or the Health Utility Index (HUI-2, HUI-3) 
(Bansback et al., 2005). By running the model over a number of cycles, a QALY score or total costs can be calculated. However, there are two types of adjustments to costs and consequences that have to be considered: discounting and a half-cycle correction.

It is a standard practice to adjust costs and consequences for differential timing by applying a discount rate of three percent based on the recommendation of the "Panel on Cost-effectiveness in Health and Medicine" which was convened by the United States Public Health Service (Gold, Russell, Siegel, \& Weinstein, 1996). Discounting allows comparison of costs and consequences in terms of net present value. That is, discounting costs and effects captures the economic theory that people prefer to consume more goods in the present than in the future. This notion of time preference could significantly affect cost-effectiveness ratios, especially given the disability and time path of disease associated with RA (Bansback et al., 2005). Markov models assume that transition occurs between cycles and that patient membership is constant for the duration of the cycle. However, in reality, patients are moving between different phases of the disease continuously. Thus, instead of assuming that patients transition between states at the end of the cycle, a half-cycle correction is employed. The half-cycle correction is based on the assumption that, on average, patients will transition between states half way through the cycle.

To evaluate a Markov model, matrix algebra is used as a cohort simulation or as a first order Monte Carlo simulation. Cohort simulation considers a hypothetical cohort of patients all beginning the process in the initial disease state at time zero, or with 
some distributions between the model states. At each cycle, based on the appropriate transition probabilities, distribution of patients in each state of the Markov model is adjusted. By running the model for many cycles, one can predict the number of patients in each state of the model over time. As discussed earlier, weights based on QALYs and costs are attached to different states for each cycle. Summing the cycle QALYs and costs across all cycles of the model gives QALYs and cost estimates for the overall cohort of patients.

Unlike cohort simulation, in first order Monte Carlo simulation, a large number of patients are followed through the model individually. The transition probabilities for cohort simulation and individual patients are the same but since an individual patient can be in only one state at a given time, s/he may or may not transit between states in any given cycle. Hence, the path of individual patients will differ based on random variation. Thus, the sum of results of $n$ simulations of the individual patients through the model will give estimates for costs and outcomes of the model (Briggs \& Sculpher, 1998; Sonnenberg \& Beck, 1993). Although mean costs and outcomes obtained in a first order Monte Carlo simulation will be similar to those obtained by cohort simulation, an advantage of Monte Carlo simulation is that estimates of variance may also be determined generating a degree of uncertainty associated with the derived costs and outcomes.

\section{Markov models for Rheumatoid Arthritis}

Two studies have used Markov models to study the progression of RA. Kobelt 
and colleagues (2002) used a Markov simulation model to assess the cost-effectiveness of treatments that affect progression of RA. Hypothetical treatment interventions were simulated to illustrate the model. Costs were calculated from data of resource utilization and patients' work capacity while utilities were assessed using the EuroQol instrument. The authors concluded that the model was a valuable tool in assessing the cost-effectiveness of different interventions in RA. A similar study used decision modeling and Markov methods to model treatment strategies for patients with RA (Albert, Aksentijevich, Hurst, Fries, \& Wolfe, 2000). Only traditional DMARDs were compared for their effectiveness. MTX was found to be superior to other DMARDs. The authors agreed that Markov analysis generated a more realistic appraisal of the outcome of the drugs as compared to standard decision tree analysis.

\section{Effectiveness of TNF Inhibitors (adalimumab, etanercept, and infliximab) and leflunomide}

Among the traditional DMARDs used to treat RA, MTX has increasingly become the treatment of choice because of its early onset of action and superior efficacy and tolerability (Weinblatt et al., 2003; Weinblatt et al., 1999). Even though MTX has been found to be effective in RA, many patients continue to have persistent disease and experience less than 50\% improvement despite receiving therapeutic doses of MTX. To increase the clinical response in such patients, rheumatologists frequently add another DMARD (Kremer, 2001). A number of studies have reported the clinical efficacy of new agents such as adalimumab, etanercept, infliximab, and leflunomide in combination with MTX. 


\section{Adalimumab}

Two double blind RCTs investigating the clinical efficacy of adalimumab in patients with RA and an inadequate response to MTX have been published (Keystone et al. 2004; Weinblatt et al., 2003). A 24-week, randomized, double-blind, placebocontrolled study (ARMADA Trial: Anti-TNF Research Study Program of the Monoclonal Antibody [D2E7] in Patients with RA) involved 271 patients with active RA (Weinblatt et al., 2003). The primary efficacy end point was the ACR criteria for $20 \%$ improvement (ACR 20) at 24 weeks. Secondary efficacy endpoints were ACR 50 and ACR 70 response rates. The patients were randomized to one of the four treatment groups, adalimumab $20 \mathrm{mg}(\mathrm{n}=69), 40 \mathrm{mg}(\mathrm{n}=67)$, or $80 \mathrm{mg}(\mathrm{n}=73)$ or placebo $(\mathrm{n}=62)$ administered as a SC injection every other week. The patients continued to take their long-term stable dosage of MTX (12.5-25 mg). An ACR 20 response at week 24 was achieved by a significantly greater proportion of patients in $20 \mathrm{mg}(47.8 \%), 40 \mathrm{mg}$ $(67.2 \%)$, and $80 \mathrm{mg}(65.8 \%)$ than in placebo plus MTX group $(14.5 \%, \mathrm{p}<.001)$. ACR 50 response rates with all three doses of adalimumab and ACR 70 response rates with 40 and $80 \mathrm{mg}$ of adalimumab were significantly greater than placebo (Weinblatt et al., 2003).

A second study by Keystone and colleagues (2004) was a multicenter, 52-week, double-blind, placebo-controlled study involving 619 patients with active RA with an inadequate response to MTX. Patients were randomized to receive adalimumab $20 \mathrm{mg}$ SC every week ( $n=212), 40$ mg SC every other week $(n=207)$, or placebo $(n=200)$ plus concomitant MTX (12.5-25 mg). Adalimumab (20 mg and $40 \mathrm{mg}$ ) was more effective 
than placebo at inhibiting the progression of structural joint damage $(\mathrm{p} \leq 0.001)$. At week 24 and 52, patients receiving $40 \mathrm{mg}$ of adalimumab achieved ACR 20 response rates of $65 \%$ and $59 \%$, respectively, versus $30 \%$ and $24 \%$ in the placebo group. Also, there was an improvement in physical function in patients receiving adalimumab.

Discontinuations were lower in the adalimumab group (22\%) compared with placebo group $(30 \%)$. However, proportion of patients reporting serious infections was higher in adalimumab (3.8\%) compared to placebo $(0.5 \%)$.

\section{Etanercept}

Three clinical studies support the efficacy of etanercept in patients with RA with an inadequate response to MTX. In a 24-week, double-blind study, Weinblatt and colleagues (1999) randomly assigned 89 patients to placebo or etanercept (25 mg SC twice a week) plus concomitant MTX. At week 24, an ACR 20 response was achieved by a significantly greater proportion of patients taking etanercept $25 \mathrm{mg}(71 \%)$ than in the placebo plus MTX group $(14.5 \% ; \mathrm{p}<.001)$. Also, the intervention group achieved ACR 50 and 70 response rates of $39 \%$ and 15\%, respectively, compared with $3 \%$ and $0 \%$, respectively, for the placebo group. All measures of disease activity were significantly improved in the etanercept group, and the only adverse events associated with this therapy were injection-site reaction.

In another double-blind, randomized trial (TEMPO: Trial of Etanercept and Methotrexate with radiographic Outcomes) 686 patients were randomly allocated to receive etanercept (25 $\mathrm{mg}$ SC twice a week), MTX (up to $20 \mathrm{mg}$ every week) or a 
combination of etanercept and MTX (Klareskog et al., 2004). The combination of etanercept and MTX was significantly better in reduction of disease activity, improvement in functional disability, and inhibition of radiographic progression compared with MTX and etanercept alone. The number of patients reporting infections or adverse events was similar in all groups.

Lan and colleagues (2004) evaluated etanercept $25 \mathrm{mg}$ given SC twice a week in patients with active RA who were maintained on MTX therapy (12.5-20 mg per week). Patients were randomized in the 12 -week, double-blind study to receive etanercept $(n=29)$ or placebo $(n=29)$. Results for the overall improvement in disease activity assessed by ACR 20 (90\% vs. 34\%), ACR 50 (66\% vs. 10\%), and ACR 70 (24\% vs. $0 \%$ ) all favored the etanercept plus MTX group. The number of patients reporting adverse events was comparable between the two treatments.

\section{Infliximab}

The efficacy of infliximab was studied using a large multi-center, doubleblinded, placebo-controlled trial (ATTRACT: Anti-Tumor Necrosis Factor Trial) involving 428 patients with active RA not responding to MTX therapy (Maini et al., 1999). Patients were randomized to receive infliximab (3mg/kg or $10 \mathrm{mg} / \mathrm{kg})$ or placebo every four to eight weeks, in addition to a stable dose of MTX (15 mg per week). After 30 weeks of therapy, $50 \%$ of patients treated with infliximab plus MTX (3mg/kg every eight weeks) achieved the ACR 20 response criteria compared with 20\% of patients receiving placebo IV injections $(\mathrm{p}<0.001)$. Clinical response was rapid and 
over half the responders attained $20 \%$ improvement by the second week of follow-up. Headaches and upper respiratory tract infections were the most frequently reported adverse events, and infusion reaction was seen in $16-20 \%$ of the patients receiving infliximab plus MTX compared with placebo (10\%).

The patients in the ATTRACT trial were followed for 54 weeks to determine the effect of infliximab and MTX on radiographic progression of the disease (Lipsky et al., 2000). Results of the study indicated that the combination provided sustained clinical benefit and halted the progression of joint damage not only in patients with limited joint damage, but also in those with extensive damage. The combination was also well tolerated and safe.

\section{Leflunomide}

The combination of leflunomide and MTX was studied in a 30-patient openlabel study (Weinblatt, Kremer, Coblyn, et al., 1999). Patients reported an ACR 20 response rate of $56 \%$ and ACR 50 response rate of $36 \%$. These responses were sustained at week 48. The leflunomide plus MTX combination was well tolerated although an increase in hepatic enzymes was observed.

The results of the open label study were confirmed in a randomized, doubleblind placebo-controlled study (Kremer et al., 2002). Addition of leflunomide to MTX provided substantial therapeutic benefit compared with adding placebo and it was generally well tolerated. Following this study, additional information on efficacy and 
safety data on the combination drug was gathered using a 24-week open-label extension study. Response to therapy was maintained through 48 weeks of treatment in patients who continued on leflunomide and MTX during the extension. ACR 20 response rates after 24 weeks of leflunomide therapy were similar between patients switched from placebo to leflunomide.

In summary, these agents have been proven to be effective and safe in appropriate and well-conducted clinical trials. In addition, the combination of adalimumab, etanercept, infliximab, or leflunomide with MTX have been shown to be better in reducing disease activity, improving functional disability, and inhibiting radiographic progression. Only one observational study has been reported comparing a combination of TNF inhibitor plus MTX with TNF inhibitor alone or MTX alone. Analysis of the Stockholm TNF- $\alpha$ Follow-Up Registry (STURE) data showed that patients receiving combination of etanercept and MTX had better outcomes compared with those patients receiving etanercept alone, thereby indicating that for patients receiving etanercept as monotherapy and having only partially satisfactory responses, adding MTX might give additional benefits (van Vollenhoven, Ernestam, Harju, and Klareskog, 2003).

\section{Economics of TNF Inhibitors (adalimumab, etanercept, and infliximab) and leflunomide}

\section{Cost analyses}

A study by Nuijten and colleagues (2001) conducted in the Netherlands estimated the total cost of etanercept therapy to be lower than those of infliximab (US 
$\$ 12,648$ vs. US $\$ 18,046$, respectively). The efficacy of the two drugs was assumed to be equivalent. The difference in the cost was mainly due to the additional costs of administration of infliximab in outpatient clinics and concomitant use of MTX although the annual drug costs was similar for both the drugs NLG 31,334 (US \$12,534) for etanercept and NLG 31,526 (US \$12,610) for infliximab. As discussed earlier, this type of analysis is a partial evaluation and ignores the long-term outcomes.

\section{Cost-effectiveness studies: Short-term studies}

In the Cost Evaluation of Novel Therapeutics in Rheumatoid Arthritis (CENTRA) study, a six-month decision tree model was designed to determine and compare the costs and effectiveness of three single agents (a hypothetical novel biologic agent, MTX, and parenteral gold) for the treatment of patients with established RA (Kavanaugh et al., 1996). Both direct costs of treating RA and indirect costs incurred by patients as a result of the disease were included in the evaluation. The authors reported that the total costs of MTX and parenteral gold were substantially lower than those of the hypothetical biological agent. However, sensitivity analyses indicated that by increasing the clinical efficacy of the hypothetical biological agent, total costs decreased due to the accrual of fewer indirect costs.

Another study by Choi and colleagues (2000) assessed the relative costeffectiveness of six different treatment options for patients with RA in whom MTX therapy had failed: etanercept monotherapy, etanercept plus MTX, cyclosporine plus MTX, triple therapy with hydroxychloroquine, sulfasalazine and MTX, continued MTX 
monotherapy, and no second-line therapy. A decision tree model with a time horizon of six months was used and an incremental cost-effectiveness ratio (ICER) for achieving ACR 20 and 70 responses were calculated. Etanercept plus MTX, the most efficacious therapy, had a higher incremental cost compared with MTX therapy. Triple therapy had the lowest ICER per ACR 20 or ACR 70 response (US $\$ 1,500$ and US $\$ 3,100$, respectively). The next less costly and more effective treatment option, etanercept plus MTX, was the most effective. The ICER was estimated to be US $\$ 42,600$ and US $\$ 34,800$, respectively. A limitation of this study was that it did not account for patients withdrawing from the treatments. As mentioned earlier, neglecting to incorporate early withdrawals could potentially bias the results.

Two studies evaluated the cost-effectiveness of TNF inhibitors with and without concomitant MTX using ACR response criteria (ACR 20 and ACR 50) as the endpoint over one year. The first study evaluated the cost-effectiveness of etanercept and adalimumab with and without concomitant MTX in RA patients. Etanercept was found to be more cost-effective than adalimumab. The cost per patient achieving ACR 20 and ACR 50 response criteria for etanercept monotherapy (25mg twice weekly) was US $\$ 29,369$ and US $\$ 43,319$, respectively, and for adalimumab (40 mg once every other week) US $\$ 40,672$ and US $\$ 78,643$, respectively. The cost per patient achieving ACR 20 and ACR 50 response criteria for etanercept plus MTX was US \$26,167 and US $\$ 47,636$, respectively; and adalimumab plus MTX was US $\$ 29,468$ and US $\$ 47,605$, respectively (Malone et al., 2003). 
The second study reported similar results in the cost-efficacy analysis of etanercept, infliximab, adalimumab, and anakinra with and without concomitant MTX. Etanercept with and without concomitant MTX was more cost-effective followed by adalimumab, anakinra, and infliximab (Chiou et al., 2003). As pointed out earlier, the short study duration of the four studies mentioned above is a major limitation. A longterm model that considers the downstream economic costs is necessary to determine the most cost-effective treatment in patients who inadequately respond to MTX alone.

\section{Cost-effectiveness studies: Long-term studies}

Despite promising short-term results from RCTs for newer TNF inhibitors that have demonstrated significant effects on functional status and radiographic progression, very few economic studies have addressed the issue of effectiveness of these drugs in RA (Brennan et al., 2003; Kobelt et al., 2003; Wong et al., 2002). This could be attributed to the lack of sufficient longitudinal data to determine whether such an increased expenditure will eventually be offset by lower total costs of the disease. A recent cost-effectiveness analysis based on the ATTRACT trial demonstrated that infliximab plus MTX was more cost-effective in the treatment of patients with RA than MTX alone (Wong et al., 2002). A Markov simulation model was used to project the 54-week results from the ATTRACT trial into lifetime economic and clinical outcomes based on the Arthritis, Rheumatism and Aging Medical Information System (ARAMIS). When compared with MTX alone, infliximab plus MTX decreased the likelihood of having advanced disability from $23 \%$ to $11 \%$ at the end of 54 weeks that 
projected to a lifetime marginal cost-effectiveness ratio of $\$ 30,500$ per discounted QALY gained.

Another study by Kobelt and colleagues (2003) used the results from the ATTRACT trial to examine the cost per QALY of infliximab plus MTX compared with MTX alone in patients with RA. A Markov simulation model assessed the progression of RA using ten-year data from two epidemiological studies conducted in Sweden and the United Kingdom (UK). The results indicated that one or two years of infliximab treatment reduced direct and indirect resource consumption in both countries. The cost per QALY gained was Swedish Krone (SEK) 32,000 (3,440 Euros) in Sweden and Great Britain Pounds (GBP) 21,600 (34,800 Euros) in UK for one-year treatment. The respective QALY gains were 0.248 and 0.298 . With two years of treatment, the costs per QALY gained were SEK 150,000 (16,100 Euros) in Sweden and GBP 29,900 $(48,200$ Euros) in the UK. However, the problem with both studies was that they modeled nonresponders to stay on infliximab for one entire year. This potentially biased the results since the patients were not responding to infliximab treatment yet were accruing high costs associated with one year of infliximab treatment.

A UK cost-utility analysis indicated that etanercept was more cost-effective than infliximab in the treatment of patients who had failed DMARD therapy. The primary clinical outcome used in the study was the HAQ disability score. The study model defined success as the achievement of ACR 20 responses and this was extrapolated beyond the time horizon of the clinical trial, to the expected lifetime of the 
cohort, by incorporating withdrawal rates from other clinical investigations. The discounted cost per QALY for etanercept therapy was $£ 18,938$ (US \$27,271) when direct costs were considered and $£ 8,439$ (US $\$ 12,152$ ) when indirect costs were considered (Brennan et al., 2001).

\section{Cost-effectiveness studies: Comparing treatment strategies}

Although a number of economic analyses demonstrate average trends for populations and provide some direction for prescribing, they are based on a number of assumptions such as similarity in baseline characteristics of patient population, clinically relevant outcome measures, variation in treatment practice, and other generalizations which may or may not be correct (Emery, 2004). In RA modeling, it is essential that time-dependent variables such as age, duration, and severity of disease be matched between data sources (Bansback et al., 2005). Thus, to find the most costeffective option for an individual patient, studies that investigate the relative costeffectiveness of long-term strategies can be useful.

A study conducted in the Netherlands determined the cost-effectiveness of five different treatment strategies over five years. A Markov simulation model was used with Markov states based on a Disease Activity Score (DAS). The treatment strategies compared included: (1) usual treatment (MTX); (2) treatment with leflunomide (in case of non-response, start usual treatment); (3) TNF inhibitors (in case of non-response, start usual treatment); (4) treatment with leflunomide (in case of non-response, start TNF inhibitors, in case of non-response, start usual treatment); (5) TNF inhibitors (in 
case of non-response, start with leflunomide, in case of non-response, start usual treatment). Expected costs and QALYs were compared between strategies and the ICER was calculated. The expected effect on QALY and disease activity was better for strategies three, four, and five than for strategies one and two. A treatment strategy starting with leflunomide followed by TNF inhibitors in case of non-response was the most cost-effective (Welsing et al., 2004).

A study by Brennan and colleagues (2003) looked at the cost-effectiveness of etanercept monotherapy under British Society for Rheumatological guidelines. The study compared a traditional DMARD sequence with etanercept third line against the same sequence excluding etanercept. The method involved simulating HAQ disability scores for 10,000 patients' lifetimes (Monte Carlo Simulation) using clinical trial data and other published data. The primary analysis included drug costs, monitoring and hospitalization. The cost per QALY was estimated to be $£ 16,330$ (US \$23,515) suggesting that etanercept was cost-effective when compared with non-biologic agents. Sensitivity analysis ( $£ 7,800$ to $£ 42,000$, US $\$ 11,232$ to $\$ 60,480)$ showed that long term HAQ progression for etanercept, DMARDs, and being a nonresponder were the most sensitive variables.

Using a method similar to that described by Brennan and colleagues (2003), Bansback and colleagues (2004) looked at the cost-utility of adalimumab in treating patients with moderate to severe RA who have failed at least two traditional DMARDs. The study implemented Swedish unit costs and treatment guidelines from a lifetime 
perspective and compared adalimumab with traditional DMARDs and other TNF inhibitors. The primary outcome measure, QALYs, was calculated from the adalimumab trial results. The method involved simulating the experiences of 10,000 hypothetical moderate to severe RA patients for each strategy using clinical trial data and other published data. The cost per QALY for both adalimumab and etanercept was estimated to be between 35,000-42,000 euros suggesting that adalimumab was at least as cost-effective as the other TNF inhibitors.

Two more studies have been conducted by the National Coordinating Center for Health Technology Assessment (NCCHTA; Barton, Jobanputra, Wilson, Bryan, \& Burls, 2004; Jobanputra et al., 2002) to assess the additional costs and QALY gain associated with the use of either etanercept or infliximab as the third DMARD in a sequence of traditional DMARDs. The first study resulted in an ICER of approximately $£ 83,000$ (US \$119,520) per QALY for etanercept and approximately $£ 115,000$ (US $\$ 165,600$ ) per QALY for infliximab. However, the effects of DMARDs on joint replacement, hospitalization, mortality, and QoL were not studied. The model used in the previous study was restructured in a subsequent study and the analysis was conducted again based on the DMARDs strategies used by rheumatologists in the UK. An ICER of approximately $£ 59,289$ (US \$85,376) per QALY for etanercept and approximately $£ 76,233$ (US \$109,775) per QALY for infliximab was reported.

In summary, none of the studies have evaluated the incremental costeffectiveness of the TNF inhibitors (adalimumab, etanercept, and infliximab) and 
leflunomide used in combination with MTX in the treatment of patients with RA who inadequately respond to MTX alone.

\section{Rheumatologists' Preferences Regarding TNF Inhibitors}

In the past, few studies have been conducted to ascertain the prescribing patterns of rheumatologists. The results of these studies reflect the changing prescribing patterns of the rheumatologists, indicating a shift from time- and severitybased approaches to patient preference-based approach. Disease modifying antirheumatic drugs have been the standard treatment for RA. Studies to determine the prescribing patterns of DMARDs show wide variations in dosing and monitoring schedules (Pope et al., 2002). Because of its favorable efficacy/toxicity trade-off, MTX has been rated as substantially more effective than any other DMARD by rheumatologists (Maetzel et al., 1998; Wolfe et al., 1998). A recent study determined the current DMARD preferences of UK consultant rheumatologists and confirmed earlier findings that MTX was the drug of choice while newer agents such as TNF inhibitors and leflunomide had replaced the older traditional DMARDs (Jobanputra et al., 2004). However, with a growing number of patients not responding to MTX, coupled with the development of highly effective TNF inhibitors, researchers have yet to assess how rheumatologists are prescribing these new drugs.

A number of questions remain unanswered regarding the use of TNF inhibitors in clinical practice. It is not clear in which patients with RA that the TNF inhibitors are being used. The approved indication for these drugs is in patients with moderate to 
severe RA. However, with their ability to reduce radiographic damage, it is not clear if these drugs are being used in patients with early RA (Kavanaugh et al; 2004). Another question should address how these powerful, new agents are being used. A number of RCTs (ARMADA, ATTRACT, and TEMPO) have shown that a combination of TNF inhibitors with MTX is superior to either drug as monotherapy in patients with active RA, but no long-term follow-up data is available to confirm the findings. Another important question a clinician faces is whether to prescribe a new TNF inhibitor if the patient has failed one such agent. A review by van Vollenhoven (2004) on switching among TNF inhibitors indicated that even though a majority of studies conducted in rheumatology clinics supported the use of alternative TNF inhibitors, caution has to be exercised in interpreting the results. The study samples were small and there were differences in the concurrent use of MTX in patients enrolled in the studies. Thus, until studies are designed to identify the predictors of response, use of TNF inhibitors will be governed by clinical judgment and accumulated experience.

Another concern regarding the use of TNF inhibitors is the potential for adverse events such as infections and lymphomas in patients who are using these drugs. Appropriately screening and monitoring patients can minimize these complications. But there are no monitoring protocols established for these drugs One study has investigated the monitoring practices of rheumatologists for MTX-, etanercept-, infliximab-, and anakinra-associated adverse events (Yazici et al., 2003). A majority (76\% to $78 \%$ ) of rheumatologists reported that they ordered ACR recommended 
screening tests for MTX but were not aware of any guidelines for monitoring TNF inhibitors.

Despite the clinical effectiveness of TNF inhibitors, there are a number of factors which influence the choice of these drugs. The drug cost is significant and their reimbursement varies greatly depending on the age of the patient, section of the country, and third party payer. Drug cost was reported to play a dominant role in rheumatologists' choice of treatment regimens (Erkan, Yazici, Harrison, \& Paget, 2002). Additionally, the different routes of administration of etanercept, infliximab, and adalimumab have resulted in different coverage and reimbursement policies (Gallup, 2001). Medicare covers only infliximab since it reimburses for intravenous infusion performed in the physicians' office. However, for patients under 65 , insurance plans insist on documentation for MTX failure before etanercept, infliximab or adalimumab can be used (Kremer, 2001). Medicaid also restricts payment for these drugs to those patients who do not demonstrate an adequate clinical response to a full dose of MTX. Self-injectable drugs such as etanercept and adalimumab are covered by pharmacy benefits (Gallup, 2001). Due to these requirements, physicians and their staff are compelled to spend more time on paper work and phone calls.

Despite the increasing use of TNF inhibitors in clinical practice, there are no studies conducted that report the current rheumatologists' prescribing patterns and the potential barriers they face in terms of using these drugs or making a choice among the currently available three TNF inhibitors. 


\section{CHAPTER THREE}

\section{METHODS}

The study was conducted in two phases. In phase I, a lifetime model was constructed using decision analytic techniques to evaluate the total costs and effects of the TNF inhibitors (adalimumab, etanercept, and infliximab) and leflunomide, used in combination with MTX, in patients with RA that inadequately respond to MTX alone. In phase II, the study assessed the current prescribing patterns of rheumatologists using TNF inhibitors to treat patients with RA through a national mail survey. The survey also assessed laboratory monitoring practices, frequency of tests ordered, and perceived barriers of rheumatologists prescribing these drugs.

\section{Phase I}

The following sections describe the design of the economic model, operationalizing the model, data used in the model, analytical methods, model assumptions and rationale that were utilized in this phase of the study.

\section{Design of the Model}

The study involved the development of a Markov model using DATA 4.0 TreeAge ${ }^{\mathrm{TM}}$ software (Data TreeAge Software, Inc., 2002). This model was used to evaluate the incremental cost-effectiveness of different treatment strategies in patients with RA that inadequately respond to MTX alone. 
The five treatment strategies compared using a decision analytic method included 1) adalimumab plus MTX, 2) etanercept plus MTX, 3) infliximab plus MTX, 4) leflunomide plus MTX, and 5) a standard therapy of MTX alone. The main analytical plan was to calculate incremental cost-effectiveness ratios. This approach involved combining cumulative measures of costs over time with a cumulative measure of effectiveness, resulting in incremental costs per clinical benefit gained. A societal perspective was taken in the estimation of costs in the base-case analysis. The total costs of therapy with each agent was composed of direct costs associated with treating MTX-resistant RA combined with indirect costs incurred by patients as a result of the disease.

The effectiveness measure consisted of American College of Rheumatology $20 \%$ response criteria (ACR 20) and quality adjusted life years (QALYs). An advantage of using ACR outcomes in cost-effectiveness analysis is their availability from publications of RCTs, and that these outcomes can be used to compare interventions within RA. However, it is difficult to make an absolute statement about whether any given option is cost-effective compared with other widely accepted costeffective interventions in medicine. To overcome this limitation, a generic measure of effectiveness such as QALY was used. The QALY combines QoL with time by adjusting life-years with a quality weight, measured as a utility. 


\section{Operationalizing the Model}

Outcomes Measure in Rheumatoid Arthritis Clinical Trials (OMERACT) acknowledges that the underlying characteristics of a clinical trial population define which population the economic analysis is applicable to (Bansback et al., 2005). Most reported efficacy data pertain to subjects in the 50- to 60-year-old age group since this patient population is most representative of the patients with RA receiving TNF inhibitors and leflunomide. Therefore, a cohort of 55-year-old women was selected as the base-case population. The hypothetical cohort included 10,000 women who had already failed two DMARDs, one of which was MTX.

The efficacy data were based on five double blinded, randomized controlled trials conducted in patients with inadequate responses to MTX (Keystone et al., 2004; Kremer et al., 2002; Maini et al., 1999; Weinblatt et al., 2003; Weinblatt et al., 1999). The baseline patient characteristics across the RCTs were similar, especially the duration of RA, HAQ disability score, and the number of previous traditional DMARDs used (Table 1).

American College of Rheumatology 20\% response data (ACR 20) for all treatment options were available in each clinical trial. The efficacy represents the probability of achieving ACR response on each drug subtracting the effect of placebo from among those who have not improved on placebo (Choi, Seeger, \& Kuntz, 2002; Choi et al., 2000). Thus, efficacy estimates were calculated for all treatment options. 
Table 1. A review of published RCT results for TNF inhibitors and leflunomide

\begin{tabular}{|c|c|c|c|c|c|c|c|c|c|}
\hline & $\mathbf{N}$ & $\begin{array}{l}\text { Baseline } \\
\text { HAQ } \\
\text { (0-3) }\end{array}$ & $\begin{array}{l}\text { HAQ at } \\
24 \text { weeks } \\
(0-3)\end{array}$ & $\begin{array}{l}\text { Previous no. } \\
\text { DMARDs }\end{array}$ & $\begin{array}{l}\text { Disease } \\
\text { duration } \\
\text { (yrs) }\end{array}$ & $\begin{array}{l}\text { Avg. } \\
\text { age } \\
\text { (yrs) }\end{array}$ & $\begin{array}{c}\text { Females } \\
(\%)\end{array}$ & $\begin{array}{l}\text { \% Patients } \\
\text { Responding } \\
\text { ACR20 }\end{array}$ & Reference \\
\hline \multicolumn{10}{|l|}{ Adalimumab } \\
\hline $40 \mathrm{mg}$ eow $+\mathrm{MTX}$ & 67 & 1.6 & 0.9 & 2.9 & 12.2 & 55.5 & 76.8 & 67 & Weinblatt et al., 2003 \\
\hline 40mg eow+MTX & 207 & 1.5 & 0.9 & 2.4 & 11.0 & 56.1 & 76.3 & 62 & Keystone et al., 2004 \\
\hline \multicolumn{10}{|l|}{ Etanercept } \\
\hline $25 \mathrm{mg} 2 \mathrm{x}$ wkly+MTX & 59 & 1.5 & 0.8 & 2.7 & 13.0 & 48.0 & 90.0 & 71 & Weinblatt et al., 1999 \\
\hline $25 \mathrm{mg} 2 \mathrm{x}$ wkly $+\mathrm{MTX}$ & 231 & NA & NA & 2.3 & 6.8 & 52.5 & 74.0 & 85 & Klareskog et al., 2004 \\
\hline \multicolumn{10}{|l|}{ Infliximab } \\
\hline $3 \mathrm{mg} / 8$ week + MTX & 86 & 1.8 & 1.5 & 2.8 & 8.4 & 56.0 & 81.0 & 50 & Maini et al., 1999 \\
\hline \multicolumn{10}{|l|}{ Leflunomide } \\
\hline $20 \mathrm{mg}$ a day+MTX & 130 & 1.5 & 1.1 & NA & 10.5 & 55.6 & 76.2 & 46 & Kremer et al., 2002 \\
\hline
\end{tabular}

$\mathrm{N}=$ number of patients enrolled in the trial

$\mathrm{NA}=$ Data not available

eow $=$ every other week

Avg. $=$ average

Wkly $=$ weekly

$\mathrm{RCT}=$ randomized clinical trial

HAQ $=$ Health Assessment Questionnaire $(0=$ No disability, $3=$ severely disabled $)$

DMARDs $=$ Disease modifying anti-rheumatic drugs

ACR 20: American College of Rheumatology Response Rate

MTX $=$ Methotrexate 
Since more than one trial provided the efficacy estimates of adalimumab plus MTX and standard therapy of MTX, average of the estimates were used in the analysis.

Treatment withdrawal rates were calculated from all RCTs by determining the proportion of patients withdrawing from the studies due to severe adverse events, lack of efficacy, or withdrawing due to other reasons. Severe adverse events included sepsis, pneumonia, or any diseases requiring hospitalization. In addition, studies were also screened for information on mild and moderate adverse events, in particular the number of patients suffering one or more mild and moderate adverse events. Mild adverse events included injection or infusion site reactions, headache, rhinitis, dizziness, abdominal pain, rash, and dyspepsia. Moderate adverse events included upper respiratory infection, pharyngitis, respiratory disorder, and sinusitis. Rate of mild and moderate adverse events was then calculated as the number of patients experiencing a mild and moderate event per patient year of follow-up for each drug.

Utilities for Markov states, such as being on medication, palliative care, and post-joint replacement were calculated from a study by Bansback and colleagues (2004). The clinical trials for all the treatments in RA report baseline and six-month HAQ disability scores. In addition, adalimumab clinical trials use Health Utility Index3 (HUI-3) as an indirect measure of health utility. Thus, an analysis of adalimumab trial data of almost 2,000 patients permitted transformation from HAQ disability scores to HUI-3 using the equation:

$$
\text { HUI-3 utility }=0.76-0.28 * \text { HAQ-DI }+0.05 * \text { Female }
$$


This transformation was necessary since etanercept, infliximab, and leflunomide trials do not report any health utility measures (Bansback et al., 2004).

\section{Operationalizing the Model for adalimumab, etanercept, infliximab, leflunomide, and MTX}

\section{Adalimumab}

The clinical efficacy of adalimumab in combination with MTX was assessed in two randomized, double-blinded studies in RA patients greater than or equal to 18 years of age with an inadequate response to MTX alone. Adalimumab (40 mg) was administered every other week subcutaneously. The results of both studies were similar; patients achieved ACR 20 rates of 63.3 to $67.2 \%$ (Keystone et al., 2004; Weinblatt et al., 2003). As described earlier, the efficacy represents the probability of achieving ACR response on each drug subtracting the effect of placebo from among those who have not improved on placebo. Thus, efficacy estimate for ACR 20 response of adalimumab in one trial was calculated to be $0.616(0.672-0.145 / 1-0.145)$ and in another was $0.479(0.633-0.295 / 1-0.295)$. An average of these two efficacy estimates (0.548) was used as the baseline probability of achieving ACR 20 improvements for adalimumab.

The proportion of patients withdrawing from the study (23.2\%) and the subset of withdrawals due to severe adverse events, lack of efficacy, and due to other reasons were estimated from the study by Keystone and colleagues (2004). The rate of mild and moderate adverse events for adalimumab was calculated to be 1.85 patients per patient-year (331 patients experiencing an event/ 179.2 patient-years of follow-up). 
Event rates cannot be directly used in the model and have to be converted to probabilities (risks) which represent the actual transitions by the cohort of individuals over a period of time (Miller et al., 1994). Probabilities (risks) are estimated in one of three ways: (1) simple cumulative method (2) actuarial method and (3) density method.

Density method was used to convert rates into probabilities (risks). This method uses group-specific rates (incidence densities (ID)) to estimate probabilities (risks) for a specified time interval (Kleinbaum, Kupper, \& Morgenstern, 1982). Thus, the $\Delta$-year probability (risk) $[\mathrm{P}(\mathrm{to}, \mathrm{t})]$ can be estimated as a function of the estimated average rate (ID) by using the following expression:

$$
\mathrm{P}_{(\mathrm{to}, \mathrm{t})}=1-\left(\mathrm{N}_{\mathrm{t}} / \mathrm{N}_{\mathrm{o}}\right)=1-\exp [-\operatorname{ID}(\Delta)]
$$

where $\mathrm{N}_{\mathrm{t}}=$ number of subjects remaining at time $t$ during the follow-up

$\mathrm{N}_{\mathrm{o}}=$ number of disease free subjects at time $t_{\mathrm{o}}$

ID = estimated average rate

$\Delta=$ elapsed time $\left(\mathrm{t}-\mathrm{t}_{\mathrm{o}}\right)$

\section{Etanercept}

The clinical efficacy of etanercept in combination with MTX was assessed in a randomized, double-blinded study involving patients with RA that responded inadequately to MTX alone. Etanercept $(25 \mathrm{mg}$ ) was administered twice every week subcutaneously. Almost $71 \%$ of patients reported achieving ACR 20 response rates (Weinblatt et al., 1999). Subtracting the placebo effect, the efficacy estimate for ACR 20 response was calculated to be 0.603 . The proportion of patients withdrawing from 
the studies (9.7\%) and the subset of withdrawals due to severe adverse events, lack of efficacy, or due to other reasons were estimated from two studies (Klareskog et al., 2004; Weinblatt et al., 1999). The rate of mild and moderate adverse events for etanercept was calculated to be 3.92 patients per patient-year.

\section{Infliximab}

The randomized, double-blinded trial assessing the clinical efficacy of infliximab (3mg/kg every 8 weeks, IV) in combination with MTX in patients with RA that inadequately respond to MTX alone reported an ACR 20 response rate of 50\% (Maini et al., 1999). After accounting for the placebo effect, the efficacy estimate for ACR 20 response was calculated to be 0.375 . The proportion of patients withdrawing from the study (26.7\%) and the subset of withdrawals due to severe adverse events, lack of efficacy, and due to other reasons were estimated from the same study. The rate of mild and moderate adverse events for infliximab was calculated to be 2.79 patients per patient-year.

\section{Leflunomide}

The 24-week randomized, double-blinded trial assessing the clinical efficacy of leflunomide (20 mg once a day, orally) in combination with MTX reported an ACR 20 response rate of 52\% (Kremer et al., 2002). Subtracting the placebo effect, the efficacy estimate for ACR 20 response was calculated to be 0.332 . The proportion of patients withdrawing from the study was $23.1 \%$ and the rate of mild and moderate adverse events for leflunomide was calculated to be 3.71 patients per patient-year. 


\section{Methotrexate}

All the RCTs included a control group receiving MTX plus placebo. The ACR 20 response rates were pooled for the five trials and, after accounting for the placebo, an average efficacy estimate of 0.19 was calculated for MTX. There was $31.2 \%$ withdrawal (pooled estimate) and the rate of mild and moderate adverse events for MTX was calculated to be 2.3 patients per patient-year. To calculate the efficacy due to MTX, the probability of achieving an ACR response due to placebo had to be calculated. The estimate for efficacy of placebo (0.11) was taken from the study by Choi and colleagues (2000) who had calculated the value from an etanercept monotherapy trial.

\section{Operationalizing the Model for Joint Replacement Therapy}

The need for a joint replacement therapy is generally perceived to reflect a failure of medical treatments. Data from short-term clinical trials of TNF inhibitors and leflunomide indicate a decrease in radiological progression of the disease; however, nothing is known about the potential long-term impact of these drugs in preventing joint replacement surgeries. A study by Wolfe and Zwillich (1998), using a database of 1,600 patients with RA, reported that $25 \%$ of the patients had joint replacement within 22 years of disease onset. The age of the study cohort was 54 years; like the baseline population in RCTs used in this study, the cohort had a baseline HAQ disability score of 1.5, disease duration of over 10 years, and had failed two to three DMARDs. Since very little published evidence is available, an assumption was made that one-fourth of the patients on palliative care will undergo joint replacement after 12 years $(22$ years 
from the disease onset) in the model. A one-time cost of $\$ 19,490$ (adjusted to 2004 dollars) was applied each time joint replacement occurred and this cost was varied by \pm $20 \%$ in the sensitivity analysis (Kremer, 2001).

\section{Operationalizing the Model for Mortality}

The OMERACT statement recommends that risk of mortality be included in decision-analytical modeling studies (Bansback et al., 2005). However, there are conflicting reports regarding the effect of RA on the risk of mortality. Some epidemiologic studies have shown an increased mortality in patients with severe RA with mean standardized mortality ratio (SMR) of 1.87 to 2.26 (Guedes, 1999; Wolfe et al., 1994). However, more recent studies have not found any effect of RA on mortality (Kobelt et al., 2003). For the baseline model, an SMR of two was considered for RA. Since some studies report that with aggressive therapy, RA mortality may be similar to that of the general population, an alternative possibility was also tested where SMR was designated as one (Wong, Ramey, \& Singh, 2001; Wong et al., 2002).

\section{Model Structure and Simulation}

The transition state model for rheumatoid arthritis is illustrated in Figure 2. The model used transition probabilities to move 10,000 different patients randomly through different Markov states over time. The time horizon of the analysis was divided into equal increments, referred to as Markov cycles, during which a patient was allowed to transition from one Markov state to another. A cycle length of six months was chosen. 
Figure 2. Transition state model

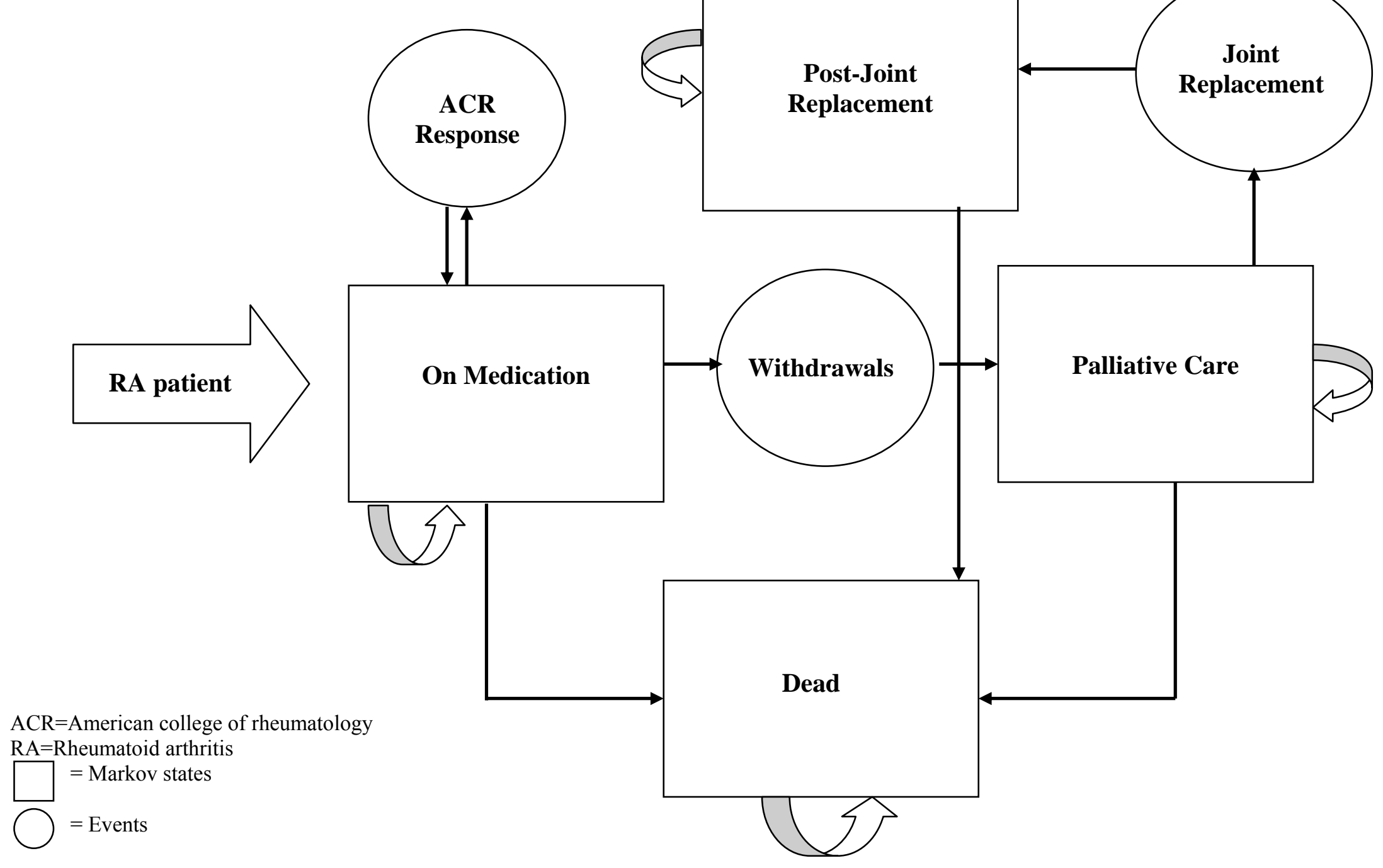


By following patients until death, the model estimated the costs and QALYs for each treatment. The analysis assumed a societal perspective (direct and indirect costs) and followed the reference case recommendations of the panel on Cost-effectiveness Analysis in Healthcare and Medicine (Gold et al., 1996). Future costs and benefits were discounted at a three percent annual rate (Gold et al., 1996).

Figure 3 shows the model structure for each strategy. The model structure for each of the five treatment arms was identical. The first branch point on the decision tree was a decision node indicating a choice of treatment being made: adalimumab plus MTX, etanercept plus MTX, infliximab plus MTX, leflunomide plus MTX, or standard therapy of MTX. Subsequent to the decision node, a Markov cycle is shown and is identical for all five treatments options.

The model had four Markov states: on medication, palliative care, post-joint replacement, and death state. In the model, individual patients started in the Markov state 'on medication'. After applying the age- and sex-adjusted mortality rate (U.S. Census Bureau, 2004), the patients experienced two events: they either continued taking the treatment or stopped the treatment. These transitions were based on prespecified transition probabilities. If the patients continued the medication, they may or may not respond to medication by at least ACR $20 \%$ response criteria. In both cases, the patients could experience mild and/or moderate adverse events that were minor enough to continue treatment. 


\section{Figure 3. Markov structure}

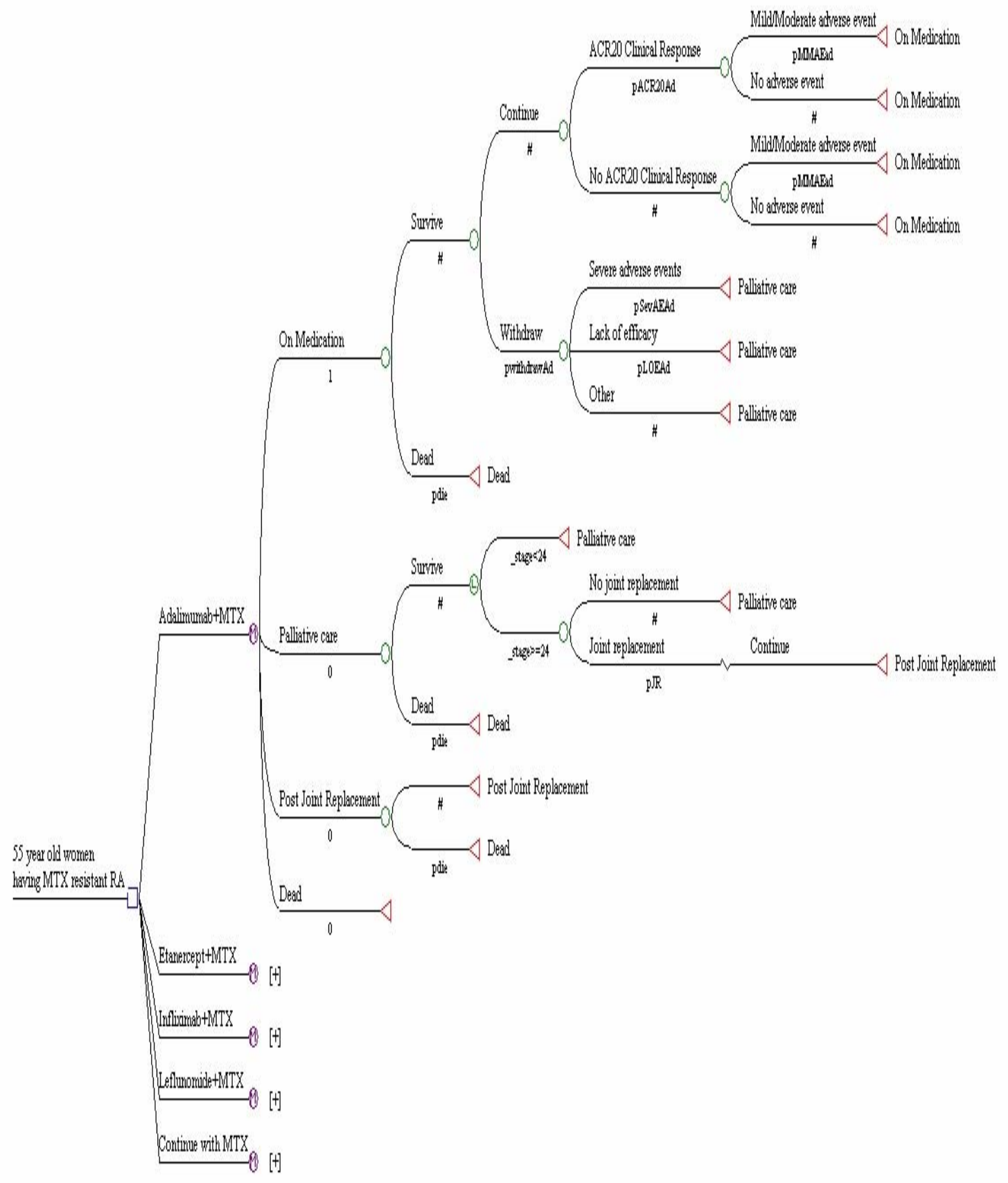


Patients who stopped the treatment did so because of the following reasons: severe adverse events, lack of efficacy, or withdrawal due to other reasons. These patients then entered the 'palliative care' state where they were treated with a combination of MTX, NSAIDs, and corticosteroids. Patients in this state were assumed to be at an increased risk of RA episodes and experienced one event: joint replacement surgery. Joint replacement surgery in patients with RA is widely perceived to result from inadequate disease control (Jobanputra et al., 2002). Once a patient had undergone joint replacement surgery, she then entered the 'post-joint replacement' state and stayed in this state for the remainder of their lifetime, eventually moving to the death state.

In the model, all the patients ultimately transitioned into the "death" state. Once the patient entered the "death" state, she could not leave that state. This, "death" state was called the absorbing state. Ideally, the model should be terminated when the entire cohort of patients are dead. However, since the Markov process is evaluated probabilitistically, the proportion of people in "non-dead" state approaches zero but is never equal to zero. Thus, an approximation was introduced to artificially terminate the cohort such that the approximation error would be minimal. Thus, in the current study, the cohort was followed until $99.999 \%$ was in the "dead" state; the remainder was treated as an error of approximation (DATA TreeAge Software manual). 


\section{Model Assumptions and Rationale}

1. The patients enrolled in the clinical trials of adalimumab, etanercept, infliximab, and leflunomide reported a previous use of two to three DMARDs. It was assumed that effectiveness and toxicity of these DMARDs were captured as a consequence on costs through increased use in health care resources and thus would be consistent across the five treatment strategies.

2. The use of NSAIDs in patients with RA was not included in the current analysis, because previous studies have shown that such use did not differ significantly between different levels of disease severity, and costs were minimal (Kobelt et al., 2003). The simulation model was focused on estimating the effects of treatments that influence the progression of the disease, rather than on calculating the precise costs of illness. Markov models are driven by the differences in costs and utilities between states and by the transition probabilities between the states. Thus, increasing or decreasing costs by the same amounts in all health states of the model will not affect the cost-effectiveness calculation. Since small costs such as NSAID use is identical in all states, these will not change the total cost of the disease significantly.

3. It was assumed that patients who did not respond to the treatments were switched to palliative care. Patients who responded, continued to stay on the medication until they experienced any severe adverse event, lack of efficacy, or withdrawal due to other reasons. Currently, there is no data to show the actual distribution of time a 
patient spends on each of these drugs. Clinical trials have reported the efficacy of these drugs over a six-month or one year period. Also, in actual clinical settings, if the patient fails to respond to one agent, rheumatologists may switch the patient to a different DMARD or to a TNF inhibitor. Patients switched to palliative care will be at an increased risk of RA episodes.

4. Clinical trials of the TNF inhibitors have shown that these drugs may cause radiological stabilization of structural joint damage. This indicates a likely decrease in future risk of joint replacement and disability. However, for those patients who stopped taking TNF inhibitors or leflunomide, there is an increased future risk of joint replacement and disability. A study by Wolfe and Zwillich (1998), using a database of 1,600 patients with RA, had reported that $25 \%$ of the patients had joint replacement within 22 years of disease onset. Since the base-case population of the model was 55 years old and had disease duration of 10 years, it was assumed that one-fourth of the population who withdrew from the TNF inhibitors and leflunomide underwent joint surgery after 12 years. A one-time joint replacement cost was applied for patients reaching this stage.

5. The evidence regarding the effect of RA on life expectancy is conflicting. Some epidemiologic studies have shown increased mortality in patients with severe RA while more recent studies have not demonstrated any effect of RA on mortality (Guedes, 1999; Kobelt et al., 2003; Wolfe et al., 1994). The study model assumed that the risk of mortality, compared with a normal age-, sex-, and race-matched 
general population, was the same. An alternative possibility was also tested where the risk of mortality was considered twice as compared with a normal age-, sex-, and race-matched general population (Wong et al., 2001; Wong et al., 2002).

\section{Data for the Model}

Three different types of data were used to populate the model:

\section{Probabilities}

The six-month transition probabilities were obtained from a formal review of the literature. The transition probabilities used in the model were calculated directly from patient-level data from active and placebo groups of the RCTs. The model assumed that after one cycle, the patients experienced the same disease progression and clinical outcomes as seen in the RCTs. Probabilities for other outcomes such as mortality and joint replacement therapy were also derived from the literature.

The base-case estimates for each variable and ranges used for sensitivity analysis are shown in Table 2. The base-case estimate for the probability of achieving ACR improvements for adalimumab was calculated from the efficacy estimate of each component using the formula:

$\mathrm{P}_{\text {Placebo }}+\left(1-\mathrm{P}_{\text {Placebo }}\right) *$ Efficacy of drug $1+\left(\mathrm{P}_{\text {Placebo }}+\left(1-\mathrm{P}_{\text {Placebo }}\right) *\right.$ Efficacy of drug 1$) *$ Efficacy of drug 2 Where $\mathrm{P}_{\text {Placebo }}$ is the proportion of patients achieving ACR response with any drug, Drug 1 = MTX (MTX is used in combination with other drugs used in all trials) Drug 2 = either adalimumab, etanercept, infliximab, or leflunomide 
Table 2. Base-case estimates (efficacy and percent probability of achieving ACR 20 response) and their ranges for sensitivity analyses: 6-month data

\begin{tabular}{|c|c|c|}
\hline Variable & Baseline estimate (range) & References \\
\hline \multicolumn{3}{|c|}{ Efficacy of individual drug ${ }^{+}$(ACR 20) } \\
\hline MTX & $19.0(16.5-21.0)$ & $\begin{array}{l}\text { Choi et al., 2000, Moreland et } \\
\text { al., 2001; Strand et al., } 1999\end{array}$ \\
\hline Adalimumab & $54.8(47.9-61.6)$ & $\begin{array}{l}\text { Keystone et al., 2004; } \\
\text { Weinblatt et al., } 2003\end{array}$ \\
\hline Etanercept & $60.3( \pm 20 \%)$ & Weinblatt et al., 1999 \\
\hline Infliximab & $37.5( \pm 20 \%)$ & Maini et al., 1999 \\
\hline Leflunomide & $33.2( \pm 20 \%)$ & Kremer et al., 2002 \\
\hline \multicolumn{3}{|c|}{ Percent probability of achieving ACR 20 response $^{++}$} \\
\hline Placebo & $11.0(9.0-13.0)$ & Choi et al., 2000 \\
\hline MTX & $27.9(25.7-29.7)$ & $\begin{array}{l}\text { Choi et al., 2000, Moreland et } \\
\text { al., 2001; Strand et al., } 1999\end{array}$ \\
\hline Adalimumab & $67.4(62.4-72.3)$ & $\begin{array}{l}\text { Keystone et al., 2004; } \\
\text { Weinblatt et al., } 2003\end{array}$ \\
\hline Etanercept & $71.4( \pm 20 \%)$ & Weinblatt et al., 1999 \\
\hline Infliximab & $54.9( \pm 20 \%)$ & Maini et al., 1999 \\
\hline Leflunomide & $51.8( \pm 20 \%)$ & Kremer et al., 2002 \\
\hline
\end{tabular}

MTX = Methotrexate

ACR 20: American College of Rheumatology Response Rate

${ }^{+}$The efficacy data is the net drug effect after adjusting for the placebo effect in each trial

Efficacy represents the probability of achieving ACR response on each drug subtracting the effect of placebo from among those who have not improved on

placebo (Choi et al., 2000)

(Drug-Placebo)/ (1-Placebo)

${ }^{++}$Probability of achieving ACR response: $\mathrm{P}_{\text {Placebo }}+\left(1-\mathrm{P}_{\text {Placebo }}\right) *$ Efficacy of drug $1+\left(\mathrm{P}_{\text {Placebo }}+\left(1-\mathrm{P}_{\text {Placebo }}\right) *\right.$ Efficacy of drug 1$) *$ Efficacy of drug $2($ Choi et al., 2000) 
For example, when treated with adalimumab and MTX, the proportion of patients achieving ACR 20 response was $0.674[0.11+(1-0.11) * 0.19+(0.11+(1-0.11) *$ $0.19) * 0.548]$. This method of calculating the ACR response implicitly assumed the additive pharmacologic effect of each component in a therapeutic option (Choi et al., 2000). The same formula was applied to calculate the proportion of patients achieving ACR 20 responses for etanercept, infliximab, leflunomide, and MTX.

Probabilities of withdrawal from treatment and the subset of withdrawals due to severe adverse events, lack of efficacy, and due to other reasons were estimated from the respective clinical trials (Table 3). In RCTs, both the mild and moderate adverse events (reported as number of patients with events), and the total number of patientyears of follow-up, are reported. Rate of mild and moderate adverse events were calculated from the information (number of patients with events / patient-years) and this was converted to probabilities using the density method described earlier (Table 4).

\section{Utilities}

Utilities are numerical values assigned to health states, which reflect the desirability of living in a given state. Health state utilities range from perfect health (weighted 1.0) to death (weighted 0 ). The weights are multiplied by the amount of time spent in each health state, and these products are summed over the patient's lifetime in the different states to obtain an estimate of QALYs. Utilities for different states in the Markov model were derived from a study by Bansback and colleagues (2004) and are reported in Table 5. 
Table 3. Base-case estimates (total withdrawals and subset of withdrawals) and their ranges for sensitivity analyses: 6-month data

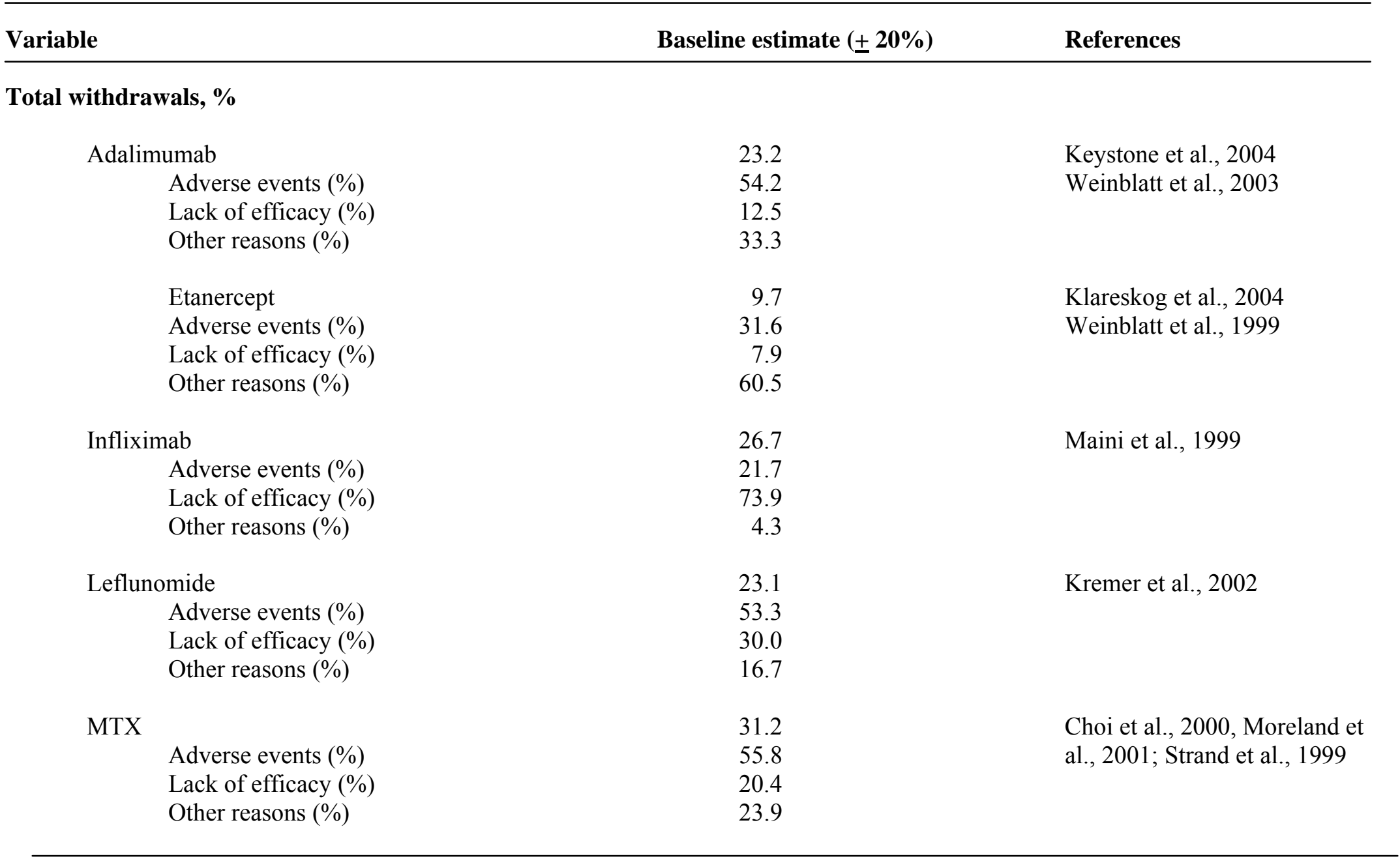


Table 4. Base-case estimates (mild/moderate adverse event) and their ranges for sensitivity analyses: 6-month data

\begin{tabular}{|c|c|c|c|}
\hline \multirow[t]{2}{*}{ Variable } & \multicolumn{2}{|r|}{ Baseline estimate $( \pm 20 \%)$} & \multirow[t]{2}{*}{ References } \\
\hline & Rate $^{\#}$ & Probability & \\
\hline \multicolumn{4}{|c|}{ Mild/moderate adverse event } \\
\hline Adalimumab & 1.85 & 0.6035 & Keystone et al., 2004 \\
\hline Etanercept & 3.92 & 0.8591 & Weinblatt et al., 1999 \\
\hline Infliximab & 2.79 & 0.7522 & Maini et al., 1999 \\
\hline Leflunomide & 3.71 & 0.8435 & Kremer et al., 2002 \\
\hline MTX & 2.30 & 0.6830 & $\begin{array}{l}\text { Choi et al., 2000, Moreland et } \\
\text { al., 2001; Strand et al., } 1999\end{array}$ \\
\hline
\end{tabular}

${ }^{*}$ Rate expressed as number of patients with events per patient year MTX=Methotrexate 
Table 5. Utilities for Markov states used in the decision analysis model ${ }^{\text {\# }}$

\begin{tabular}{cc}
\hline Markov States & Utilities $\mathbf{( + \mathbf { 2 0 } \% )}$ \\
\hline On Medication & \\
Adalimumab+MTX & $0.558(0.446-0.669)$ \\
Etanercept+MTX & $0.586(0.469-0.703)$ \\
Infliximab+MTX & $0.460(0.368-0.552)$ \\
Leflunomide+MTX & $0.502(0.402-0.602)$ \\
Standard MTX Therapy & $0.446(0.357-0.535)$ \\
Palliative Care & $0.283(0.227-0.340)$ \\
Post-Joint Replacement & $0.152(0.122-0.182)$ \\
\hline
\end{tabular}

\# Utilities were estimated using an equation derived by Bansback and Colleagues (2004): HUI-3 $=0.76-0.28 * \mathrm{HAQ}$ DI $+0.05 *$ Female MTX=Methotrexate 
Utilities for the five different treatments were calculated based on the HAQ disability score at six-months derived from their respective RCTs using the equation:

HUI-3 utility $=0.76-0.28 *$ HAQ-DI $+0.05 *$ Female

HUI-3 = Health Utility Index-3

HAQ-DI= Health Assessment Questionnaire Disability Score

Since the patients who withdrew from the treatments and moved to the palliative care or post-joint replacement states were assumed to experience an increase in RA episodes, it was decided that after six months there will be a $25 \%$ increase in their baseline HAQ disability score. For the palliative care state, the baseline HAQ disability score was assumed to be 1.50 ; for the post-joint replacement state, the baseline HAQ disability score was assumed to be 1.88 . The increase in the HAQ disability score at six months was then substituted in the above equation to generate utilities for each of these two states.

\section{Costs}

Direct costs: The costs of each of the TNF inhibitors, leflunomide, and MTX included the drug costs, costs of monitoring, costs of treating mild and/or moderate and severe adverse events arising from the treatment, and the cost of joint replacement therapy (Table 6). Drug costs were calculated from Drug Topics Red Book (2004). The Red Book lists the average wholesale price (AWP) for prescribed medications. Drug costs were based on the standard dose derived from the package insert of the drug. In 
Table 6. Costs: 6-month data used in the decision analysis model for each drug

\begin{tabular}{|c|c|c|c|c|c|}
\hline Variables & Methotrexate & Adalimumab & Etanercept & Infliximab & Leflunomide \\
\hline Dose & $15 \mathrm{mg} /$ week & $\begin{array}{l}40 \mathrm{mg} \text { every } \\
\text { other week }\end{array}$ & $\begin{array}{l}25 \mathrm{mg} \text { twice } \\
\text { every week }\end{array}$ & $\begin{array}{l}3 \mathrm{mg} / \mathrm{kg} \\
\text { every } 8 \text { weeks }\end{array}$ & $\begin{array}{l}20 \mathrm{mg} / \\
\text { day }\end{array}$ \\
\hline \multicolumn{6}{|l|}{ Direct Costs (\$) ${ }^{\#}$} \\
\hline Medication costs ${ }^{\S}$ & $\$ 714.8( \pm 20 \%)$ & $\$ 7,894.8( \pm 20 \%)$ & $\$ 7,894.8( \pm 20 \%)$ & $\$ 8,520.6^{*}( \pm 20 \%)$ & $\$ 2,233.9( \pm 20 \%)$ \\
\hline Administration cost ${ }^{+}$ & - & - & - & \multicolumn{2}{|c|}{$\$ 848.6(\$ 663.6-\$ 1,033.6)$} \\
\hline $\begin{array}{l}\text { Monitoring costs } \\
\text { (First 6-month) }\end{array}$ & $\begin{array}{l}\$ 707.7 \\
(\$ 488.4-\$ 927)\end{array}$ & $\begin{array}{l}\$ 996.5 \\
(\$ 730.5-\$ 1,262.5)\end{array}$ & $\begin{array}{l}\$ 996.5 \\
(\$ 730.5-\$ 1,262.5)\end{array}$ & $\begin{array}{l}\$ 996.5 \\
(\$ 730.5-\$ 1,262.5)\end{array}$ & $\begin{array}{c}\$ 670 \\
(\$ 487.4-\$ 852.5)\end{array}$ \\
\hline $\begin{array}{l}\text { Monitoring costs }{ }^{\S \S} \\
\text { (After 6-month) }\end{array}$ & $\begin{array}{l}\$ 404.4 \\
(\$ 299.6-\$ 501.2)\end{array}$ & $\begin{array}{l}\$ 354.5 \\
(\$ 240.2-\$ 468.8)\end{array}$ & $\begin{array}{l}\$ 354.5 \\
(\$ 240.2-\$ 468.8)\end{array}$ & $\begin{array}{l}\$ 354.5 \\
(\$ 240.2-\$ 468.8)\end{array}$ & $\begin{array}{c}\$ 473.2 \\
(\$ 347.9-\$ 598.4)\end{array}$ \\
\hline $\begin{array}{l}\text { Costs of treating severe } \\
\text { Adverse Event }\end{array}$ & $\$ 8,500( \pm 20 \%)$ & $\$ 8,500( \pm 20 \%)$ & $\$ 8,500( \pm 20 \%)$ & $\$ 8,500( \pm 20 \%)$ & $\$ 8,500( \pm 20 \%)$ \\
\hline $\begin{array}{l}\text { Costs of treating mild/ } \\
\text { moderate adverse event }\end{array}$ & $\begin{array}{l}\$ 165.3 \\
(\$ 126.2-\$ 204.3)\end{array}$ & $\begin{array}{l}\$ 165.3 \\
(\$ 126.2-\$ 204.3)\end{array}$ & $\begin{array}{l}\$ 165.3 \\
(\$ 126.2-\$ 204.3)\end{array}$ & $\begin{array}{l}\$ 165.3 \\
(\$ 126.2-\$ 204.3)\end{array}$ & $\begin{array}{l}\$ 165.3 \\
(\$ 126.2-\$ 204.3)\end{array}$ \\
\hline
\end{tabular}

\footnotetext{
\# All costs adjusted to 2004 dollars

$\S$ Based on Drug Topics Red Book (AWP 2004)

$\S \S$ Based on Medicare and Managed Care reimbursement rates

${ }^{*}$ Mean dose per infusion $=2.8$ vials, number of infusion per 6 -month $=4.4$

${ }^{+}$Medicare rate (2004) $\$ 150.8$ per infusion (First hour infusion rate $=\$ 117.79$, Every additional hour $=\$ 33.02$ ); Administration cost $=150.8 * 4.4=\$ 663.6$

Cost of treating mild/moderate adverse event $=1$ office visit $+\mathrm{CBC}+\mathrm{LFT}+$ Urine analysis + Chest X-ray +10 day antibiotic treatment

Medicare:http://www.cms.hhs.gov/providers/pufdownload/default.asp?

Managed care: http://www.purdue.edu/hr/Benefits/mac.htm
} 
addition, infliximab is administered as an infusion and the administration cost was added to the treatment costs. All five drugs required intensive monitoring in the first six months, after which routine monitoring was required. Thus, the model incorporated higher monitoring costs in the first six months and lower constant monitoring costs after the first six months. Monitoring costs were identified by applying Medicare and managed care current laboratory fees to the recommended laboratory schedule. An average of the two costs was included in the model (Tables 6 and 7).

The cost of drug-related severe adverse events was based on the cost of treating pneumonia, which is the most commonly reported severe adverse event due to treatment with TNF inhibitors. The cost of treating pneumonia $(\$ 8,500)$ was derived from the reimbursement rates paid by Medicare. Treatment of mild and/or moderate events was assumed to result in an office visit, a complete blood count test (CBC), one liver function test (LFT), one urine analysis, a chest X-ray, and a ten day antibiotic treatment. The costs of physician visit and laboratory tests were identified by applying Medicare and managed care current lab fees. The antibiotic cost was calculated from the Drug Topics Red Book (2004).

Joint replacement therapy was treated as an event and a one-time cost was applied at the time of joint replacement. The estimate $(\$ 19,490)$ came from the published literature (Kremer, 2001). For patients in palliative care, there was no startup cost, but a constant cost of $\$ 1,141$ per six-months was applied. This cost included the six-month average cost of NSAIDs, corticosteroids, and MTX. The costs of 
Table 7. Monitoring costs: 6-month data used in the decision analysis model for each drug

\begin{tabular}{|c|c|c|c|c|c|c|c|}
\hline Variables & CPT & $\begin{array}{l}\quad \text { Unit Rate (\$) } \\
\text { Managed care/ Medicare } \\
\text { Reimbursement Rates }\end{array}$ & $\begin{array}{l}\text { Methotrexate } \\
\text { (Frequency) } \\
\mathbf{1}^{\text {st }} \text { 6-mth/ } \\
\text { After 6-mth }\end{array}$ & $\begin{array}{l}\text { Adalimumab } \\
\text { (Frequency) } \\
1^{\text {st }} \text { 6-mth/ } \\
\text { After 6-mth }\end{array}$ & $\begin{array}{l}\text { Etanercept } \\
\text { (Frequency) } \\
1^{\text {st }} \text { 6-mth/ } \\
\text { After 6-mth }\end{array}$ & $\begin{array}{l}\text { Infliximab } \\
\text { (Frequency) } \\
1^{\text {st }} \text { 6-mth/ } \\
\text { After 6-mth }\end{array}$ & $\begin{array}{l}\text { Leflunomide } \\
\text { (Frequency) } \\
\mathbf{1}^{\text {st }} \mathbf{6} \text { mth/ } \\
\text { After 6-mt }\end{array}$ \\
\hline Chest X-ray & 71020 & $\$ 65.6 / \$ 36.3$ & 1 & 1 & 1 & 1 & 1 \\
\hline Complete blood count & 85007 & $\$ 9.6 / \$ 4.8$ & $7 / 6$ & $13 / 6$ & $13 / 6$ & $13 / 6$ & $7 / 6$ \\
\hline Urine analysis & 81005 & $\$ 6.1 / \$ 3.0$ & 2 & 13 & 13 & 13 & - \\
\hline Creatinine & 82575 & $\$ 26.4 / \$ 13.2$ & 7 & 13 & 13 & 13 & 7 \\
\hline \multicolumn{8}{|l|}{ LFTs } \\
\hline ALT & 84460 & $\$ 7.3 / \$ 7.4$ & $7 / 6$ & $1 / 1$ & $1 / 1$ & $1 / 1$ & $7 / 6$ \\
\hline $\mathrm{AST}$ & 84450 & $\$ 7.1 / \$ 7.2$ & $7 / 6$ & $1 / 1$ & $1 / 1$ & $1 / 1$ & $7 / 6$ \\
\hline Albumin & 82040 & $\$ 11.5 / \$ 6.9$ & $7 / 6$ & $1 / 1$ & $1 / 1$ & $1 / 1$ & $7 / 6$ \\
\hline Protein purified test & 86580 & $2 \$ 2.07 /-$ & - & 1 & 1 & 1 & - \\
\hline Antinuclear antibody & 86039 & $\$ 31.2 / \$ 15.6$ & 2 & $13 / 1$ & $13 / 1$ & $13 / 1$ & $-/ 1$ \\
\hline Rheumatologist (visits) & & $\$ 72.1 / \$ 35.5$ & $3.3 / 3.3$ & $3.3 / 3.3$ & $3.3 / 3.3$ & $3.3 / 3.3$ & $3.3 / 3.3$ \\
\hline Nonrheumatologist (visits & & $\$ 72.1 / \$ 35.5$ & $1.6 / 1.6$ & $1.6 / 1.6$ & $1.6 / 1.6$ & $1.6 / 1.6$ & $1.6 / 1.6$ \\
\hline \multicolumn{3}{|c|}{ Total monitoring costs (First 6-month, Range) } & $\$ 488.4-\$ 927$ & $\$ 730.5-\$ 1,262.5$ & $\$ 730.5-\$ 1,262.5$ & $\$ 730.5-\$ 1,262.5$ & $\$ 730.5-\$ 1,262.5$ \\
\hline \multicolumn{3}{|c|}{ Average monitoring costs (First 6-month) } & $\$ 707.7$ & $\$ 996.5$ & $\$ 996.5$ & $\$ 996.5$ & $\$ 670$ \\
\hline \multicolumn{3}{|c|}{ Total monitoring costs (After 6-month, Range) } & $\$ 299.6-\$ 501.2$ & $\$ 240.2-\$ 468.8$ & $\$ 240.2-\$ 468.8$ & $\$ 240.2-\$ 468.8$ & $\$ 347.9-\$ 598.4$ \\
\hline \multicolumn{3}{|c|}{ Average monitoring costs (First 6-month) } & $\$ 404.4$ & $\$ 354.5$ & $\$ 354.5$ & $\$ 354.5$ & $\$ 473.2$ \\
\hline
\end{tabular}


NSAIDs and corticosteroids were calculated from the Drug Topics Red Book (2004).

Patients in the post-joint replacement state were assumed to have the same cost of treatment as those in the palliative care state. The costs of hospitalizations due to RArelated surgeries were calculated by using an exponential equation which is a function of the HAQ disability score (Choi et al., 2000; Table 8).

$$
\text { Cost of surgery }=635.5 \mathrm{e}^{1.0935 \text { (HAQ score) }}
$$

where HAQ score is the score at 24 week

Indirect costs: Indirect costs were included to capture potential savings associated with improvement in RA with each treatment. Studies have shown that indirect costs in RA patients are one to three times the direct costs (Meenan, Yelin, Henke, Curtis, \& Epstein, 1978; Stone, 1984). In the present study, indirect costs was calculated as one to three times that of direct costs.

\section{Adjusting Costs}

When costs are taken from the literature or from electronic datasets, they often require adjustments before they can be included in the cost-effectiveness analysis. When older costs are used, they underestimate the cost of medical care in present-day terms, unless they are adjusted for inflation (Muennig, 2002). Thus, all the costs that were taken from the literature in this study were adjusted to 2004 dollars. The annual changes in medical inflation between 1990 and 2004 are reported by the Bureau of Labor Statistics. The proportional increase in the prices was then calculated using the formula: (high year index value - low year index value)/low year index value 
Table 8. Base-case estimates and their ranges for sensitivity analyses: 6-month data

\begin{tabular}{lcc}
\hline Variables & Baseline estimate (range ) & References \\
\hline Hospitalization costs $^{+++}$ & & Choi et al., 2000 \\
MTX & $\$ 1302.7( \pm 20 \%)$ & \\
Adalimumab & $\$ 859.8( \pm 20 \%)$ & \\
Etanercept & $\$ 762.0( \pm 20 \%)$ & Kremer, 2001 \\
Infliximab & $\$ 1639.1( \pm 20 \%)$ & \\
Leflunomide & $\$ 928.2( \pm 20 \%)$ & \\
Joint replacement cost & $\$ 19,490.0( \pm 20 \%)$ & \\
Palliative care cost & $\$ 1141.0( \pm 20 \%)$ & \\
\hline
\end{tabular}

${ }^{+++}$Hospitalization costs calculated using the formula:

Cost of surgery $=635.5 \mathrm{e}^{1.0935 \text { (HAQ score) }}$ where HAQ score is the score at 24 week

MTX $=$ methotrexate

All costs adjusted to 2004 dollars 


\section{Discounting Future Costs}

Medical interventions often result in decreased future medical costs that must be accounted for in present-day terms. Based on the recommendation of the "Panel on Cost-effectiveness in Health and Medicine" which was convened by the United States Public Health Service, all future costs and outcomes were discounted into their net present value by three percent before they were included in the cost-effectiveness analysis (Gold et al., 1996). The general formula for discounting future costs used was:

$$
\text { Cost of future event/ }(1+\text { discount rate })^{\text {years }}
$$

\section{Analysis}

The base-case analysis represented the expected average effectiveness and costs per patient population, discounted at three percent for a hypothetical cohort of 10,000 patients. A Markov Model can be evaluated by using a Markov cohort simulation where the patients in the cohort are followed as they move among the predetermined Markov states. Also, the Markov Model can be evaluated by using a first order Monte Carlo simulation. In the first order Monte Carlo simulation, a large number of patients are followed through the model individually. A single simulation trial will randomly select a path at each uncertainty, based on the probability of each outcome's occurrence. Thus, the path followed by different patients will differ by chance (Shaw \& Zachary, 2002).

The baseline model in this study was analyzed using a first order Monte Carlo simulation. Even though costs and outcomes estimated by the two methods are similar, 
an advantage of using a first order Monte Carlo simulation is that estimates of variance associated with costs and outcomes in each arm of the model are also determined, giving a measure of uncertainty of the derived costs and outcomes. Estimated average costs and QALYs were then used to calculate an incremental cost-effectiveness ratio (ICER). An ICER was calculated as the additional cost per patient achieving a particular outcome, compared with the next more expensive option.

The use of tracker variables in the Markov Model also dictated the use of a first order Monte Carlo simulation. A tracker variable is a special type of variable that can only be used in Monte Carlo simulation trials and not during Markov cohort simulation. The primary function of a tracker variable is to serve as a memory within a Markov process. For example, if a patient encounters a node containing the tracker variable definition numEvents=numEvents +1 , DATA will take the current global value of numEvents for that patient's trial, add one to it, and store the new global value (DATA TreeAge Software manual). Two tracker variables were created in the model. The tracker variable 'Number_JR' was created to count the number of joint replacements a patient underwent in the model. Another tracker variable 'Number_JRage' was created to estimate the age at which the patient underwent joint replacement.

Sensitivity analysis is used to deal with uncertainty in the model parameters. Sensitivity analysis was performed by varying baseline variables over a plausible range to test the robustness of the model over a range of assumptions and probability estimates. One-way sensitivity analysis was used to vary one parameter at a time from 
its baseline value and the analyses used a first order Monte Carlo simulation to observe the effect on the choice of strategy. Because the overall variability of a model is poorly characterized by one-way sensitivity analysis, a second order Monte Carlo simulation (probabilistic sensitivity analysis) was also used to characterize this variability.

In second order Monte Carlo simulation, each parameter in the model, instead of taking on a single value, was assumed to be a variable quantity with a known range of possible values and an associated range of distribution functions. For a cohort of patients, all the model parameters were allowed to vary within assigned ranges according to an assigned distribution and the average cost and effect of each treatment strategy was calculated. This process was repeated a large number of times to generate empirical distributions for mean costs and effects of each treatment strategy (Shaw \& Zachary, 2002).

\section{Phase II}

Primary data for phase II was collected using a survey instrument. The survey was developed to determine rheumatologists' current prescribing patterns, laboratory monitoring practices, and perceived barriers in prescribing the three TNF inhibitors. The following section describes selection of the study population, development and administration of the survey instrument, data collection, and data analytical techniques that were used for this phase of the study.

\section{Study Population and Sample Selection}

The research design used for this phase of the study was a cross-sectional 
survey design. The population of interest was a national sample of rheumatologists. A mailing list of all the rheumatologists in the United States was obtained from SK\&A Information Services Inc., a private mailing list firm. The total number of rheumatologists obtained from SK\&A was 3,008.

\section{Sample Size Calculation}

The sample size for the study was determined on the basis of getting an estimate of the true proportion of a variable of interest in the population within $\pm 5 \%$ points with 95\% confidence (Kalton, 1987). The sample size necessary was determined using the following formula:

$$
\mathrm{n}=\frac{\mathrm{Z}^{2} * \Pi(1-\Pi)}{\mathrm{E}^{2}}
$$

n: estimate of appropriate sample size

$\mathrm{Z}$ : the number of standard errors away from the mean, or the $\mathrm{Z}$ score for the confidence interval chosen, and equals 1.96

$\Pi$ : True population proportion and is equal to $33 \%$ (considering that all three TNF inhibitors are being equally prescribed by the rheumatologists)

E: acceptable level of error in estimation of population proportion, and is equal to $5 \%$.

Substituting the values in the equation gave a sample size of 340 rheumatologists that need to be surveyed from the study population. Assuming a 20\% response rate for physician mail surveys, a total of 1,700 rheumatologists were needed to achieve the required sample size. After excluding trainees, non-clinical, and pediatric rheumatologists $(n=45)$, a total of 1,970 rheumatologists were randomly selected from 
3,008 rheumatologists and mailed a survey questionnaire. To assess the nonresponse bias, a one-page non-response questionnaire was mailed to 200 randomly selected nonresponding rheumatologists.

\section{Instrument Development and Content}

A self-administered survey was used for this phase of the study. Surveys allow information to be collected from a sample group and to be generalized to the population at large. Mail surveys are used more frequently than telephone and face-to-face interviews because they are easy to implement, incur relatively low cost, and have been found to be more reliable than telephone and face-to-face interviews (Dillman, 1999; DeLeeuw, 1992).

The survey questionnaire was designed to investigate rheumatologists' current prescribing patterns for the three TNF inhibitors in patients with RA. Specifically, the survey was designed to gather information on rheumatologists' preference for any particular TNF agent, to identify the patient population in which these agents were being used, to determine what laboratory monitoring protocols were being followed, and to identify rheumatologists' perceived barriers in prescribing these agents.

The first section of the survey questionnaire was focused on exploring rheumatologists' prescribing patterns of the three TNF inhibitors. Rheumatologists were asked to identify the patient population (newly diagnosed, mild RA, moderate RA, or severe RA) in which they prescribed each of the TNF inhibitors. 
Rheumatologists were also asked to rank, in order of preference, the TNF inhibitor they were more likely to prescribe in patients with RA. The next question in this section asked the rheumatologists to indicate how they used TNF inhibitors in patients who responded inadequately to MTX alone. The last question in this section asked the rheumatologists if they had switched any patient to a different TNF inhibitor if the first TNF inhibitor had not worked. A follow-up question required the respondents to indicate the reasons for switching.

The second section of the survey questionnaire was designed to investigate the use of adalimumab, etanercept, and infliximab by rheumatologists in their clinical practice. For each drug, respondents were asked to provide the dose and frequency of dosing they prescribed in patients with newly diagnosed, mild, moderate, and severe RA. The respondents were also asked about the laboratory tests ordered for each drug and the frequency of ordering these tests. Respondents could select from a list of five laboratory tests. These tests included anti-nuclear antibody test, complete blood count test, creatinine test, chest $\mathrm{x}$-ray, and purified protein derivative test.

The third section of the questionnaire was designed to measure rheumatologists' perceived barriers in prescribing each of the TNF inhibitors. Respondents were asked to rate factors such as insurance coverage, cost, safety and efficacy of these drugs, and route of administration, on a one to seven Likert-type scale where one was "no problem' and seven was 'major problem'. 
Demographic and practice-related information were obtained in the last section of the questionnaire. Data collected included age in years, gender, number of years in practice as a rheumatologist, primary practice site, average number of patients with RA seen per week, average number of prescriptions written per week for each of the drugs, and the monitoring guidelines (ACR, EULAR, both, or none) used by the respondents in treating patients with TNF inhibitors.

\section{Instrument Validation}

Faculty members from a School of Pharmacy and a School of Medicine were approached to assess the clarity, readability levels, and appropriateness of the instrument. All the comments provided were incorporated in order to enhance the face and content validity of the survey questionnaire. Feedback on the content of the questionnaire was also obtained from rheumatologists practicing at West Virginia University Hospital. This process helped determine the relevance of questions and clarity of instructions included in the survey. It also helped determine if any important variables were omitted or if any information was redundant.

\section{Instrument Administration}

Approvals for all survey related documents (survey questionnaire, cover letters, and non-response survey) were sought from the West Virginia University's Institutional Review Board (WVU-IRB). Once approved, the survey process was initiated by mailing a cover letter, the survey instrument, and a business reply envelope to a randomly selected national sample of rheumatologists. The cover letter (Appendices A 
\& B) explained the study, noting the importance of determining the prescribing patterns of rheumatologists and their perceived barriers in prescribing the TNF inhibitors. In addition to the purpose of the study, the letter also emphasized voluntary participation and assured confidentiality of responses. Respondents were asked to return the completed survey (Appendix C) in a self-addressed business reply envelope. The surveys were coded for follow-up purposes only.

A second mailing followed after a period of four weeks. This consisted of a cover letter, the survey instrument, and a business reply envelope; and this second packet was mailed only to those rheumatologists who did not respond to the first mailing. Finally, about ten weeks after the first mailing, a one page non-response questionnaire was mailed to a randomly selected sample of non-responding rheumatologists to assess non-response bias. The non-response survey (Appendix D) was conducted to determine if there is a difference between respondents and nonrespondents. The non-response survey assessed the reasons for not participating in the study and also collected critical demographic and prescribing-related information on the use of TNF inhibitors.

\section{Data Handling and Analysis}

All the returned surveys were checked for completeness. Incomplete responses were not included in the analyses. Data entry and statistical analyses were performed using the Statistical Package for Social Sciences ${ }^{\circledR}$ (SPSS) for Windows, Version 12.0. 
Descriptive statistics such as means and standard deviations (SDs) were used to report data. Independent t-tests, analysis of variance (ANOVA), and repeated measures ANOVA were used to assess differences among groups. Frequency distributions were generated for demographic and practice characteristics of the respondents.

This chapter discussed in detail the methodology employed to meet the study goals and to address the research questions of both phases I and II. The next chapter will present the results of both of the study phases. 


\section{CHAPTER FOUR}

\section{RESULTS AND DISCUSSION}

\section{Phase I}

In phase I, a Markov model was constructed to evaluate the total costs and effects of five treatments: adalimumab plus MTX, etanercept plus MTX, infliximab plus MTX, leflunomide plus MTX, and a standard therapy of MTX in patients with RA that inadequately respond to MTX alone. The model was evaluated using a first order Monte Carlo simulation. The patients entered the model at age 55 and moved through the three Markov states of 'on medication, 'palliative care', and 'post-joint replacement'. Each state was associated with some events such as response to treatment, withdrawal from drug therapy, or experiencing joint replacement surgery. Finally the patients entered the absorbing state (death state). Estimates of population mean costs and mean effects (e.g. QALYs) were obtained using first order Monte Carlo simulation, which were then used to calculate the ICERs.

The transition from Markov states to 'death' state can be considered as a function of two independent forces: disease-specific mortality and all-cause mortality excluding the disease under consideration. As described earlier, there are conflicting reports regarding the effect of RA on the risk of mortality. For the baseline model, a standardized mortality ratio (SMR) of one was considered for RA and therefore, age-, sex-, race-adjusted all-cause mortality rates were used. Since some studies report that the mortality due to RA may be twice that of the general population, age-, sex-, race- 
adjusted all-cause mortality rates was adjusted by a factor of two to reflect the increase in risk of mortality due to RA. The probability of dying based on age, sex, and race were obtained from the Statistical Abstract of the United States (U.S Census Bureau, Vital Statistics, 2004). These probabilities and the adjusted probabilities to reflect the increased risk in mortality due to RA are reported in Tables 9 and 10. 
Table 9. Probability of dying based on age-, sex-, and race-adjusted all-cause mortality rates

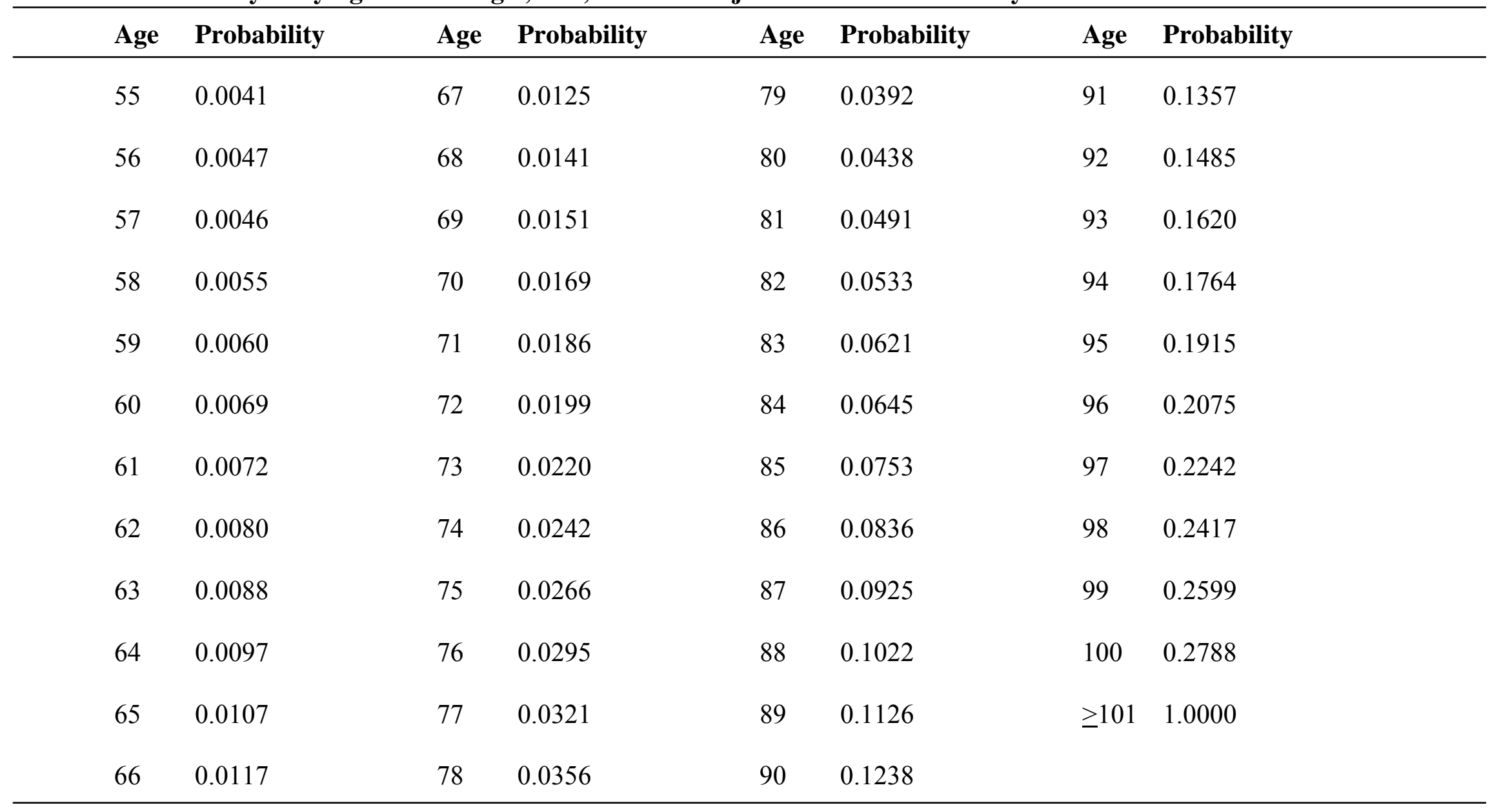

Source: Statistical Abstract of the United States (U.S Census Bureau, Vital Statistics, 2004) 
Table 10. Probability of dying based on RA-adjusted mortality rates

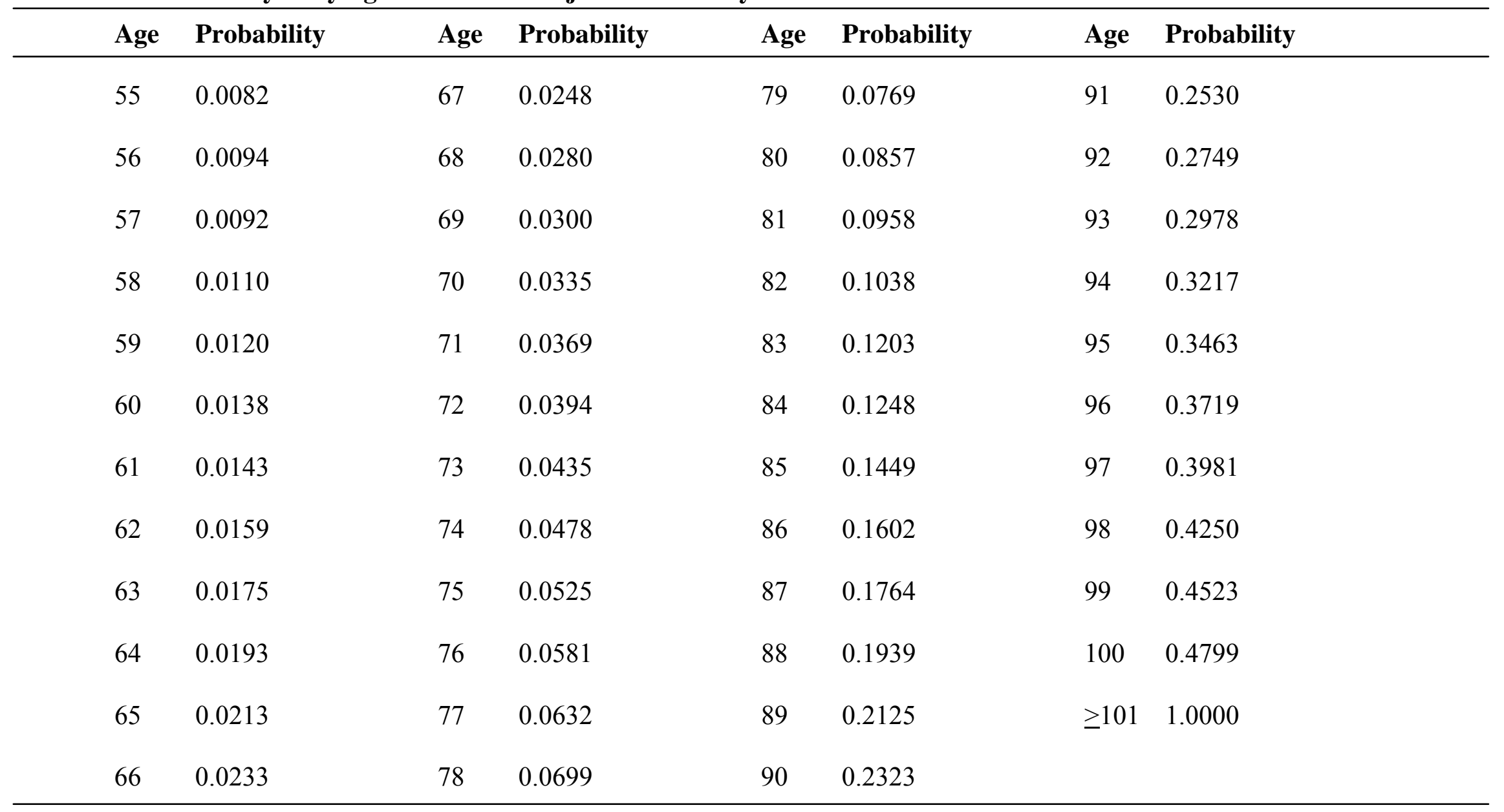

Source: Statistical Abstract of the United States (U.S Census Bureau, Vital Statistics, 2004) 


\section{Base-case Analysis Results}

Ten thousand hypothetical patients were sampled for all the five treatments using a first order Monte Carlo simulation. Natural mortality (adjusted for age, gender, and race) was incorporated into the model via standard life tables for a United States (US) population (U.S Census Bureau, Vital Statistics, 2004). A discount rate of three percent, applied to costs and benefits in the base-case analysis, was based on the recommendation of the "Panel on Cost-effectiveness in Health and Medicine" which was convened by the United States Public Health Service (Gold et al., 1996). As discussed earlier, the indirect costs due to RA are estimated to be one to three times that of the direct costs. In the base-case analysis, we assumed that the indirect costs were the same as the direct costs. Analyses were also performed assuming indirect costs at two and three times the direct costs and these results are reported later in this chapter.

Estimated lifetime mean total costs and QALYs for each treatment are reported in Table 11. The standard MTX treatment was the least expensive $(\$ 82,956$ for a lifetime treatment) and etanercept plus MTX was the most expensive treatment $(\$ 314,895)$. The two other TNF inhibitors, infliximab plus MTX $(\$ 189,145)$ and adalimumab plus MTX $(\$ 187,748)$ were less expensive than etanercept plus MTX but were more expensive than leflunomide plus MTX $(\$ 116,991)$. Etanercept plus MTX had the highest gain of 15.84 QALYs followed by adalimumab plus MTX (13.28

QALYs), leflunomide plus MTX (12.94 QALYs), infliximab plus MTX (12.51 QALYs), and standard MTX treatment (12.29 QALYs). 
Table 11. Base-case results for total costs and effectiveness using Monte Carlo simulations of 10,000 hypothetical patients with RA that inadequately respond to MTX (using age-, sex- race-adjusted all-cause mortality rates)

\begin{tabular}{|c|c|c|c|c|c|c|c|c|}
\hline Treatment Strategy & \multicolumn{4}{|c|}{ Total Costs ${ }^{*}(\$)$} & \multicolumn{4}{|c|}{ Effectiveness (QALYs) } \\
\hline Standard MTX & $82,956 \quad( \pm 25,567)$ & 1,362 & 86,420 & 168,706 & $12.29( \pm 2.93)$ & 0.22 & 13.12 & 19.00 \\
\hline Leflunomide + MTX & $116,991 \quad( \pm 52,012)$ & 2,276 & 107,578 & 338,463 & $12.94( \pm 3.18)$ & 0.25 & 13.55 & 23.24 \\
\hline Infliximab+MTX & $189,145( \pm 123,914)$ & 6,360 & 157,079 & 853,187 & $12.51( \pm 3.00)$ & 0.23 & 13.26 & 20.96 \\
\hline Adalimumab+MTX & $187,748( \pm 113,828)$ & 5,232 & 157,689 & 701,482 & $13.28( \pm 3.35)$ & 0.28 & 13.74 & 25.32 \\
\hline Etanercept+MTX & $314,895( \pm 193,655)$ & 5,184 & 272,060 & 888,545 & $15.84( \pm 4.88)$ & 0.29 & 15.49 & 36.05 \\
\hline $\begin{array}{l}{ }^{*} \text { Total costs }=\text { Direct cos } \\
\text { QALYs = Quality adjust } \\
\text { MTX = Methotrexate } \\
\text { SD = Standard deviation } \\
\text { Min = Minimum } \\
\text { Max = Maximum }\end{array}$ & $\begin{array}{l}\text { Indirect costs (Indirect } \\
\text { fe years }\end{array}$ & $\mathrm{cc}$ & ame a & & & & & \\
\hline
\end{tabular}


An incremental cost-effectiveness analysis was performed to determine whether costs incurred due to the addition of a TNF inhibitor or leflunomide to standard MTX treatment achieved any additional benefits. This involved determining the ICERs. The ICERs were calculated for both total costs and direct costs only of the treatments and are reported in Table 12.

Under base-case assumptions using total costs, etanercept plus MTX provided more health benefits and was more costly than standard MTX treatment, with a resulting cost-effectiveness ratio of $\$ 49,724 / \mathrm{QALY}$. The second most cost-effective treatment was leflunomide plus MTX with an ICER of \$52,833/QALY. Infliximab plus MTX was less effective and more costly than adalimumab plus MTX (i.e., dominated), and adalimumab plus MTX had a higher ICER and costs compared with leflunomide plus MTX (i.e., ruled out through extended dominance). The results for total costs are also represented graphically in Figure 4 where the $\mathrm{X}$-axis represents the effectiveness in QALYs and the Y-axis gives the total costs in dollars.

In the analysis including only direct costs, the dominance status of each considered treatments remained the same as in the base-case analysis using total costs consideration. In the analysis including only direct costs, the ICER of etanercept plus MTX compared with standard MTX treatment was \$24,333/QALY, while the ICER of leflunomide plus MTX compared with standard MTX treatment was \$27,717/QALY. Infliximab plus MTX was dominated by leflunomide plus MTX and adalimumab plus MTX was ruled out through extended dominance. 
Table 12. Base-case direct costs, total costs, effectiveness, and incremental cost-effectiveness ratios of different treatments for patients with RA that inadequately respond to MTX (using age-, sex-, and race-adjusted all-cause mortality rates)

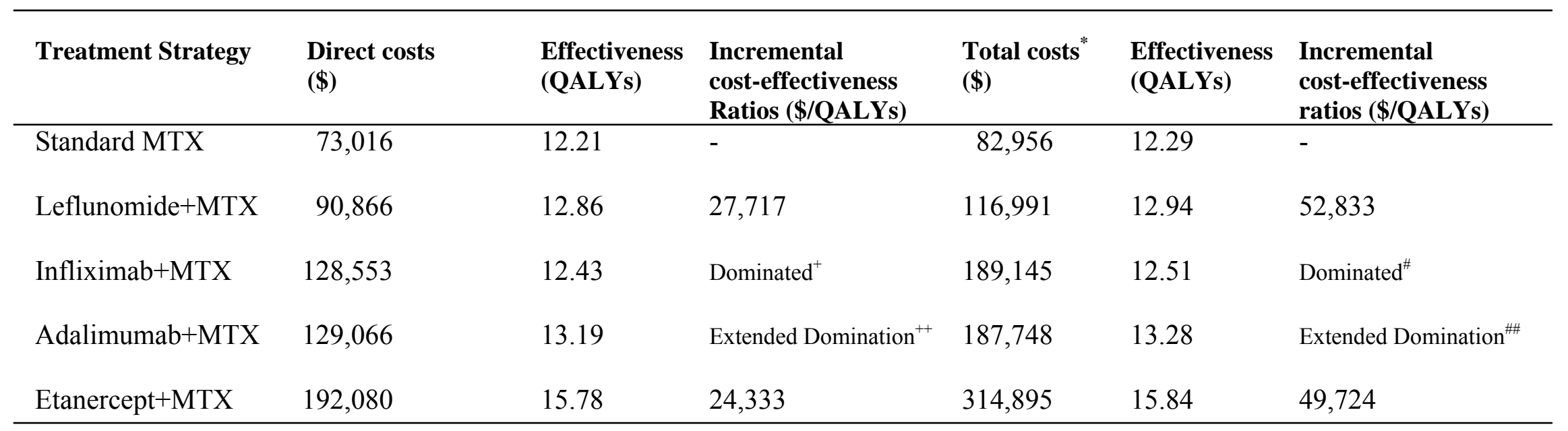

All treatments compared to the reference case: Standard MTX treatment

MTX= Methotrexate

${ }^{*}$ Total costs $=$ Direct costs + Indirect costs (Indirect costs calculated as same as direct costs)

${ }^{+}$Infliximab+MTX dominated by Leflunomide+MTX

${ }^{++}$Adalimumab+MTX dominated by a blend of Leflunomide+MTX and Etanercept+MTX with a coefficient of inequity between 0.623 and 0.884 .

\# Infliximab+MTX dominated by Adalimumab+MTX

\#\# Adalimumab+MTX dominated by a blend of Leflunomide+MTX and Etanercept+MTX with a coefficient of inequity between 0.642 and 0.881 . 
Figure 4. Total costs and effectiveness of different treatments for patients with RA that inadequately respond to MTX (using age-, sex-, race-adjusted all-cause mortality rates)

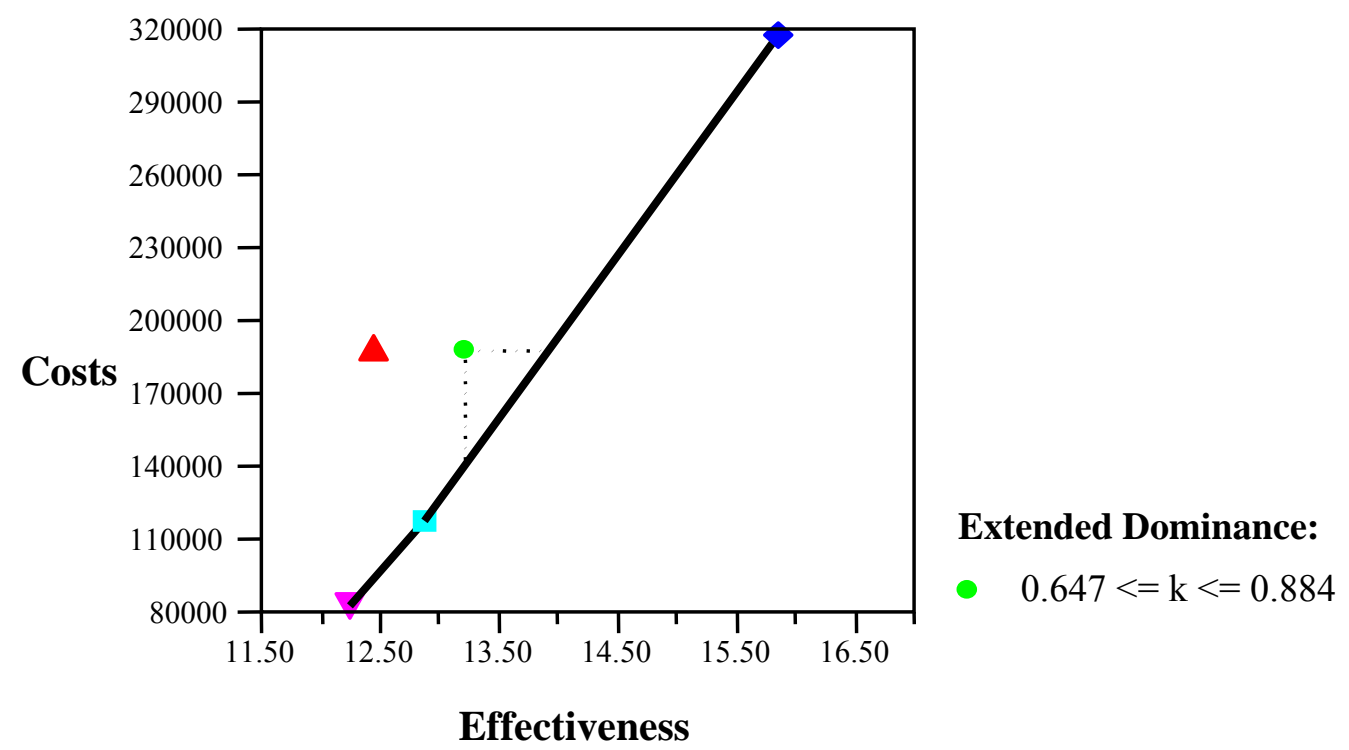

- Adalimumab+MTX

$\Delta$ Infliximab+MTX

$\nabla$ Continue with MTX

MTX $=$ methotrexate
- Etanercept+MTX

Leflunomide+MTX 


\section{Sensitivity Analysis Results}

Sensitivity analysis is used to deal with uncertainty in the model parameters. These analyses are performed to determine the robustness of the base-case results with regards to variation in the base-case estimates, both costs and effectiveness. A comparison between the original cost-effectiveness ratios and the sensitivity analysis cost-effectiveness ratios of an input parameter provides an indication of how sensitive the overall model results are to changes in that particular parameter. Thus, if the results are found to be stable over a wide range of input parameter, the model's conclusions are considered to be robust (Gold et al., 1996).

Since the baseline estimates were obtained by analyzing the model using a first order Monte Carlo simulation, one-way sensitivity analysis also employed first order Monte Carlo simulation. One-way sensitivity analysis was conducted by vary one input parameter at a time from their baseline values to observe the effect on the choice of treatment strategy. Probabilities and costs were varied between ranges or $\pm 20 \%$. Results of one-way sensitivity analyses on different input parameters and their effect on total costs, effectiveness, and ICERs are reported in Tables 13-23. The first row in the tables gives the base-case results which serve as a benchmark against which all the subsequent derived values from the sensitivity analysis can be compared. 
Table 13. One-way sensitivity analyses - Probability of withdrawals

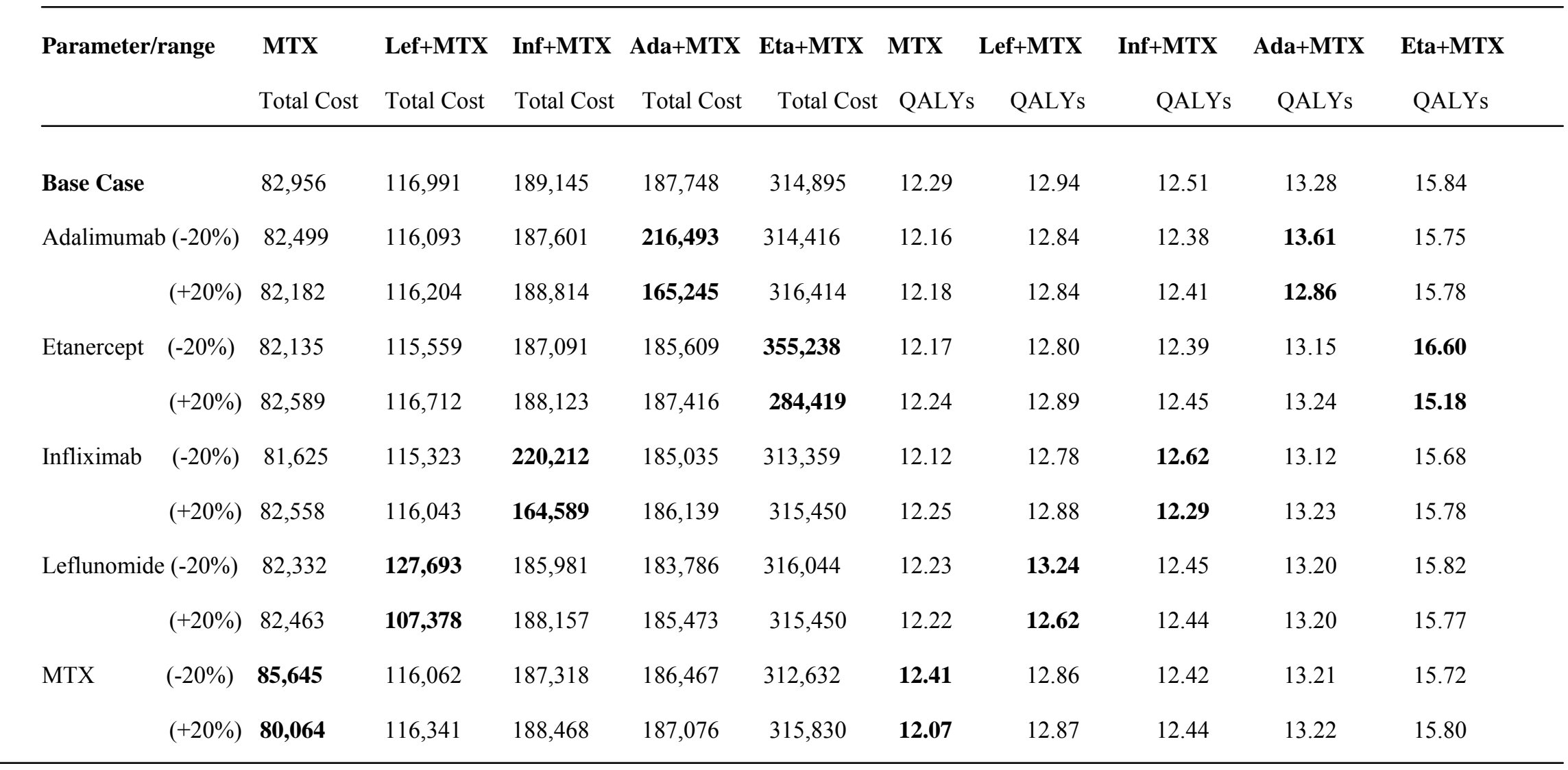

MTX: Standard MTX treatment, Lef+MTX: Leflunomide+MTX, Inf+MTX: Infliximab+MTX, Ada+MTX: Adalimumab+MTX, Eta+MTX: Etanercept+MTX 
Table 14. One-way sensitivity analyses - Probability of withdrawals due to severe adverse events

\begin{tabular}{|c|c|c|c|c|c|c|c|c|c|c|}
\hline Parameter/range & \multirow{2}{*}{$\begin{array}{l}\text { MTX } \\
\text { Total Cost }\end{array}$} & \multirow{2}{*}{$\begin{array}{l}\text { Lef+MTX } \\
\text { Total Cost }\end{array}$} & \multirow{2}{*}{$\begin{array}{l}\text { Inf }+ \text { MTX } \\
\text { Total Cost }\end{array}$} & \multirow{2}{*}{$\begin{array}{l}\text { Ada+MTX } \\
\text { Total Cost }\end{array}$} & \multirow{2}{*}{$\begin{array}{l}\text { Eta+MTX } \\
\text { Total Cost }\end{array}$} & \multirow{2}{*}{$\begin{array}{l}\text { MTX } \\
\text { QALYs }\end{array}$} & \multirow{2}{*}{$\begin{array}{r}\text { Lef+MTX } \\
\text { QALYs }\end{array}$} & \multirow{2}{*}{$\begin{array}{r}\text { Inf+MTX } \\
\text { OALYs }\end{array}$} & \multirow{2}{*}{$\begin{array}{c}\text { Ada+MTX } \\
\text { QALYs }\end{array}$} & \multirow{2}{*}{$\begin{array}{l}\text { Eta+MTX } \\
\text { QALYs }\end{array}$} \\
\hline & & & & & & & & & & \\
\hline Base Case & 82,956 & 116,991 & 189,145 & 187,748 & 314,895 & 12.29 & 12.94 & 12.51 & 13.28 & 15.84 \\
\hline Adalimumab (-20\%) & 82,333 & 116,246 & 188,343 & 185,137 & 317,377 & 12.22 & 12.86 & 12.42 & 13.20 & 15.81 \\
\hline$(+20 \%)$ & 81,806 & 115,694 & 187,136 & 187,456 & 312,667 & 12.14 & 12.81 & 12.35 & 13.15 & 15.71 \\
\hline \multirow[t]{2}{*}{ Etanercept } & 82,380 & 115,541 & 186,667 & 185,096 & 312,606 & 12.19 & 12.82 & 12.40 & 13.14 & 15.73 \\
\hline & 82,361 & 116,108 & 187,831 & 186,072 & 317,839 & 12.29 & 12.86 & 12.42 & 12.21 & 15.84 \\
\hline \multirow[t]{2}{*}{ Infliximab } & 82,245 & 116,005 & 188,077 & 186,325 & 314,690 & 12.19 & 12.82 & 12.40 & 13.17 & 15.74 \\
\hline & 82,381 & 115,791 & 189,164 & 185,566 & 315,083 & 12.23 & 12.86 & 12.43 & 13.20 & 15.80 \\
\hline Leflunomide (-20\%) & 82,498 & 115,521 & 189,128 & 186,815 & 316,097 & 12.22 & 12.88 & 12.45 & 13.22 & 15.78 \\
\hline$(+20 \%)$ & 82,320 & 116,663 & 188,163 & 185,758 & 314,707 & 12.19 & 12.83 & 12.41 & 13.18 & 15.74 \\
\hline \multirow[t]{2}{*}{ MTX } & 81,528 & 116,556 & 189,915 & 187,414 & 318,828 & 12.22 & 12.86 & 12.44 & 13.21 & 15.84 \\
\hline & 82,678 & 115,206 & 186,861 & 184,440 & 312,838 & 12.13 & 12.79 & 12.36 & 13.13 & 15.70 \\
\hline
\end{tabular}

MTX: Standard MTX treatment, Lef+MTX: Leflunomide+MTX, Inf+MTX: Infliximab+MTX, Ada+MTX: Adalimumab+MTX, Eta+MTX: Etanercept+MTX 
Table 15. One-way sensitivity analyses - Probability of mild/moderate adverse event

\begin{tabular}{|c|c|c|c|c|c|c|c|c|c|c|c|}
\hline \multirow{2}{*}{\multicolumn{2}{|c|}{ Parameter/range }} & MTX & Lef + MTX & Inf + MTX & Ada + MTX & Eta + MTX & MTX & Lef + MTX & Inf + MTX & Ada+MTX & Eta + MTX \\
\hline & & Total Cost & Total Cost & Total Cost & Total Cost & Total Cost & QALYs & QALYs & QALYs & QALYs & QALYs \\
\hline \multicolumn{2}{|l|}{$\overline{\text { Base Case }}$} & 82,956 & 116,991 & 189,145 & 187,748 & 314,895 & 12.29 & 12.94 & 12.51 & 13.28 & 15.84 \\
\hline \multicolumn{2}{|c|}{ Adalimumab (-20\%) } & 82,125 & 115,716 & 186,652 & 185,383 & 313,820 & 12.20 & 12.85 & 12.42 & 13.19 & 15.77 \\
\hline & $(+20 \%)$ & 82,115 & 115,637 & 186,411 & 185,091 & 311,861 & 12.18 & 12.85 & 12.40 & 13.18 & 15.69 \\
\hline \multirow[t]{2}{*}{ Etanercept } & $(-20 \%)$ & 82,120 & 115,228 & 185,102 & 183,887 & 312,504 & 12.19 & 12.84 & 12.41 & 13.18 & 15.78 \\
\hline & $(+20 \%)$ & 82,683 & 116,092 & 188,138 & 185,677 & 315,641 & 12.25 & 12.89 & 12.47 & 12.23 & 15.80 \\
\hline \multirow[t]{2}{*}{ Infliximab } & $(-20 \%)$ & 81,796 & 115,506 & 186,065 & 185,258 & 314,397 & 12.14 & 12.80 & 12.36 & 13.15 & 15.70 \\
\hline & $(+20 \%)$ & 81,911 & 115,602 & 185,984 & 185,479 & 316,197 & 12.17 & 12.82 & 12.38 & 13.16 & 15.77 \\
\hline \multicolumn{2}{|c|}{ Leflunomide $(-20 \%)$} & 82,175 & 116,170 & 188,102 & 187,249 & 314,811 & 12.16 & 12.83 & 12.37 & 13.17 & 15.75 \\
\hline & $(+20 \%)$ & 82,249 & 116,413 & 188,178 & 187,017 & 314,545 & 12.21 & 12.85 & 12.42 & 13.20 & 15.77 \\
\hline \multirow[t]{2}{*}{ MTX } & $(-20 \%)$ & 81,725 & 115,359 & 186,556 & 184,491 & 312,368 & 12.16 & 12.82 & 12.38 & 13.16 & 15.71 \\
\hline & $(+20 \%)$ & 82,333 & 116,608 & 189,036 & 187,461 & 315,370 & 12.19 & 12.85 & 12.41 & 13.20 & 15.77 \\
\hline
\end{tabular}

MTX: Standard MTX treatment, Lef+MTX: Leflunomide+MTX, Inf+MTX: Infliximab+MTX, Ada+MTX: Adalimumab+MTX, Eta+MTX: Etanercept+MTX 
Table 16. One-way sensitivity analyses - Probability of joint replacement and discount rate

\begin{tabular}{ccccccccccc}
\hline Parameter/range & MTX & Lef+MTX & Inf+MTX & Ada+MTX & Eta+MTX & MTX & Lef+MTX & Inf+MTX & Ada+MTX & Eta+MTX \\
& Total Cost & Total Cost & Total Cost & Total Cost & Total Cost & QALYs & QALYs & QALYs & QALYs & QALYs \\
\hline Base Case & 82,956 & 116,991 & 189,145 & 187,748 & 314,895 & 12.29 & 12.94 & 12.51 & 13.28 & 15.84 \\
Probability of joint replacement & & & & & & & & & & \\
$(0.1)$ & 79,246 & 112,750 & 183,244 & 182,448 & 311,608 & 12.56 & 13.22 & 12.80 & 13.57 & 16.14 \\
$(0.5)$ & 83,420 & 116,666 & 189,199 & 186,113 & 313,172 & 12.01 & 12.65 & 12.23 & 12.98 & 15.53 \\
Discount rate & & & & & & & & & & \\
$(0 \%)$ & 14,962 & 187,978 & 266,748 & 266,251 & 444,484 & 20.91 & 21.66 & 21.18 & 22.04 & 25.79 \\
$(5 \%)$ & 61,465 & 92,971 & 159,864 & 158,538 & 264,591 & 19.37 & 9.98 & 9.58 & 10.31 & 12.41 \\
\hline
\end{tabular}

MTX: Standard MTX treatment, Lef+MTX: Leflunomide+MTX, Inf+MTX: Infliximab+MTX, Ada+MTX: Adalimumab+MTX, Eta+MTX: Etanercept+MTX 
Table 17. One-way sensitivity analyses - Utilities: on medication, palliative care, and post-joint replacement

\begin{tabular}{|c|c|c|c|c|c|c|c|c|c|c|c|}
\hline \multirow{2}{*}{\multicolumn{2}{|c|}{ Parameter/range }} & MTX & Lef+MTX & Inf + MTX & Ada+MTX & Eta+MTX & MTX & Lef+MTX & Inf+MTX & Ada+MTX & \\
\hline & & Total Cost & Total Cost & Total Cost & Total Cost & Total Cost & QALYs & QALYs & QALYs & QALYs & QALYs \\
\hline \multicolumn{2}{|l|}{ Base Case } & 82,956 & 116,991 & 189,145 & 187,748 & 314,895 & 12.29 & 12.94 & 12.51 & 13.28 & 15.84 \\
\hline \multicolumn{12}{|c|}{ On medication Utilities } \\
\hline \multicolumn{2}{|c|}{ Adalimumab (-20\%) } & 82,968 & 116,414 & 188,832 & 186,252 & 317,218 & 12.29 & 12.91 & 12.50 & 12.56 & 15.85 \\
\hline & $(+20 \%)$ & 82,497 & 116,409 & 188,808 & 186,937 & 314,330 & 12.23 & 12.87 & 12.44 & 13.91 & 15.76 \\
\hline \multirow[t]{2}{*}{ Etanercept } & $(-20 \%)$ & 82,044 & 115,569 & 186,640 & 185,492 & 315,142 & 12.16 & 12.81 & 12.38 & 13.16 & 14.09 \\
\hline & $(+20 \%)$ & 82,673 & 115,934 & 186,438 & 185,119 & 313,793 & 12.24 & 12.88 & 12.46 & 13.22 & 17.38 \\
\hline \multirow[t]{2}{*}{ Infliximab } & $(-20 \%)$ & 81,471 & 115,652 & 186,241 & 186,061 & 313,641 & 12.14 & 12.80 & 11.86 & 13.15 & 15.72 \\
\hline & $(+20 \%)$ & 82,159 & 115,767 & 187,125 & 185,503 & 313,890 & 12.21 & 12.85 & 12.90 & 13.19 & 15.72 \\
\hline \multicolumn{2}{|c|}{ Leflunomide (-20\%) } & 82,397 & 116,099 & 188,336 & 186,637 & 315,912 & 12.20 & 12.22 & 12.43 & 13.20 & 15.78 \\
\hline & $(+20 \%)$ & 82,103 & 115,862 & 188,141 & 186,145 & 313,873 & 12.19 & 13.46 & 12.40 & 13.18 & 15.72 \\
\hline \multirow[t]{2}{*}{ MTX } & $(-20 \%)$ & 82,222 & 115,709 & 188,063 & 185,661 & 314,782 & 11.80 & 12.84 & 12.42 & 13.18 & 15.73 \\
\hline & $(+20 \%)$ & 81,917 & 115,516 & 187,661 & 185,018 & 315,897 & 12.57 & 12.83 & 12.39 & 13.17 & 15.78 \\
\hline \multirow[t]{2}{*}{ UPall. Care } & $(-20 \%)$ & 82,305 & 116,314 & 188,827 & 187,033 & 315,317 & 10.51 & 11.27 & 10.79 & 11.16 & 14.60 \\
\hline & $(+20 \%)$ & 81,822 & 115,485 & 187,255 & 185,455 & 314,426 & 13.84 & 14.40 & 14.01 & 14.74 & 16.91 \\
\hline \multirow[t]{2}{*}{ UPost-JR } & $(-20 \%)$ & 82,036 & 115,638 & 187,336 & 185,503 & 316,368 & 11.82 & 12.46 & 12.04 & 12.81 & 15.46 \\
\hline & $(+20 \%)$ & 82,389 & 116,234 & 188,648 & 186,658 & 316,882 & 12.58 & 13.22 & 12.80 & 13.56 & 16.13 \\
\hline
\end{tabular}

MTX: Standard MTX treatment, Lef+MTX: Leflunomide+MTX, Inf+MTX: Infliximab+MTX, Ada+MTX: Adalimumab+MTX, Eta+MTX: Etanercept+MTX UPall.care $=$ Palliative care utilities, UPost-JR $=$ post-joint replacement utilities 
Table 18. One-way sensitivity analyses - medication costs and infliximab administration costs

\begin{tabular}{|c|c|c|c|c|c|c|c|c|c|c|}
\hline \multirow[t]{2}{*}{ Parameter/range } & MTX & Lef+MTX & Inf + MTX & Ada+MTX & Eta + MTX & MTX & Lef+MTX & Inf+MTX & Ada+MTX & Eta+MTX \\
\hline & Total Cost & Total Cost & Total Cost & Total Cost & Total Cost & QALYs & QALYs & QALYs & QALYs & QALYs \\
\hline Base Case & 82,956 & 116,991 & 189,145 & 187,748 & 314,895 & 12.29 & 12.94 & 12.51 & 13.28 & 15.84 \\
\hline \multicolumn{11}{|l|}{$\underline{\text { Medication costs }}$} \\
\hline Adalimumab (-20\%) & 82,206 & 116,013 & 187,189 & 167,384 & 316,082 & 12.21 & 12.85 & 12.41 & 13.19 & 15.80 \\
\hline$(+20 \%)$ & 82,656 & 116,745 & 188,589 & 206,667 & 317,777 & 12.24 & 12.88 & 12.44 & 13.23 & 15.86 \\
\hline \multirow[t]{2}{*}{ Etanercept } & 81,961 & 114,731 & 188,132 & 183,286 & 272,391 & 12.19 & 12.81 & 12.39 & 13.15 & 15.76 \\
\hline & 82,392 & 116,026 & 187,046 & 186,003 & 358,282 & 12.21 & 12.85 & 12.43 & 13.19 & 15.78 \\
\hline \multirow[t]{2}{*}{ Infliximab } & 82,285 & 115,950 & 169,784 & 185,710 & 314,081 & 12.20 & 12.84 & 12.42 & 13.18 & 15.76 \\
\hline & 82,369 & 116,215 & 205,646 & 186,682 & 316,016 & 12.21 & 12.86 & 12.42 & 13.20 & 15.82 \\
\hline Leflunomide (-20\%) & 81,897 & 110,672 & 188,814 & 186,646 & 318,205 & 12.18 & 12.84 & 12.39 & 13.18 & 15.83 \\
\hline$(+20 \%)$ & 82,059 & 120,755 & 186,701 & 184,826 & 313,864 & 12.18 & 12.84 & 12.41 & 13.18 & 15.76 \\
\hline \multirow[t]{2}{*}{ MTX } & 81,617 & 114,708 & 186,923 & 185,126 & 310,428 & 12.24 & 12.88 & 12.45 & 13.23 & 15.78 \\
\hline & 83,344 & 118,521 & 190,856 & 189,643 & 319,301 & 12.20 & 12.87 & 12.43 & 13.22 & 15.79 \\
\hline \multicolumn{11}{|c|}{ Infliximab infusion administration costs } \\
\hline$(\$ 663.6)$ & 82,222 & 115,866 & 185,702 & 186,068 & 315,605 & 12.18 & 12.82 & 12.40 & 13.16 & 15.75 \\
\hline$(\$ 1,033.6)$ & 82,281 & 115,901 & 190,038 & 186,002 & 314,196 & 12.20 & 12.85 & 12.43 & 13.19 & 15.78 \\
\hline
\end{tabular}

MTX: Standard MTX treatment, Lef+MTX: Leflunomide+MTX, Inf+MTX: Infliximab+MTX, Ada+MTX: Adalimumab+MTX, Eta+MTX: Etanercept+MTX 
Table 19. One-way sensitivity analyses - Costs of monitoring in first six months

\begin{tabular}{|c|c|c|c|c|c|c|c|c|c|c|c|}
\hline \multirow{2}{*}{\multicolumn{2}{|c|}{ Parameter/range }} & MTX & Lef+MTX & Inf + MTX & Ada+MTX & Eta+MTX & MTX & Lef+MTX & Inf + MTX & Ada+MTX & Eta+MTX \\
\hline & & Total Cost & Total Cost & Total Cost & Total Cost & Total Cost & QALYs & QALYs & QALYs & QALYs & QALYs \\
\hline \multicolumn{2}{|l|}{ Base Case } & 82,956 & 116,991 & 189,145 & 187,748 & 314,895 & 12.29 & 12.94 & 12.51 & 13.28 & 15.84 \\
\hline \multicolumn{2}{|c|}{ Adalimumab (\$730) } & 81,782 & 115,293 & 185,304 & 184,320 & 313,735 & 12.17 & 12.82 & 12.38 & 13.15 & 15.72 \\
\hline & $(\$ 1,262)$ & 81,767 & 115,349 & 186,791 & 185,420 & 314,904 & 12.13 & 12.78 & 12.34 & 13.12 & 15.71 \\
\hline \multirow[t]{2}{*}{ Etanercept } & $(\$ 730)$ & 81,782 & 115,293 & 185,304 & 184,320 & 313,735 & 12.17 & 12.82 & 12.38 & 13.15 & 15.72 \\
\hline & $(\$ 1,262)$ & 81,767 & 115,349 & 186,791 & 185,420 & 314,904 & 12.13 & 12.78 & 12.34 & 13.12 & 15.71 \\
\hline \multirow[t]{2}{*}{ Infliximab } & $(\$ 730)$ & 81,782 & 115,293 & 185,304 & 184,320 & 313,735 & 12.17 & 12.82 & 12.38 & 13.15 & 15.72 \\
\hline & $(\$ 1,262)$ & 81,767 & 115,349 & 186,791 & 185,420 & 314,904 & 12.13 & 12.78 & 12.34 & 13.12 & 15.71 \\
\hline \multicolumn{2}{|c|}{ Leflunomide (\$487) } & 82,533 & 115,877 & 187,278 & 185,844 & 314,059 & 12.25 & 12.88 & 12.45 & 13.22 & 15.79 \\
\hline & $(\$ 852)$ & 82,050 & 116,057 & 188,246 & 186,393 & 316,781 & 12.18 & 12.82 & 12.40 & 13.17 & 15.79 \\
\hline \multirow[t]{2}{*}{ MTX } & $(\$ 488)$ & 81,846 & 116,127 & 187,559 & 186,872 & 316,117 & 12.15 & 12.81 & 12.36 & 13.16 & 15.76 \\
\hline & $(\$ 927)$ & 82,251 & 115,767 & 187,139 & 185,321 & 312,891 & 12.20 & 12.86 & 12.43 & 13.21 & 15.73 \\
\hline
\end{tabular}

MTX: Standard MTX treatment, Lef+MTX: Leflunomide+MTX, Inf+MTX: Infliximab+MTX, Ada+MTX: Adalimumab+MTX, Eta+MTX: Etanercept+MTX 
Table 20. One-way sensitivity analyses - Costs of monitoring after six months

\begin{tabular}{|c|c|c|c|c|c|c|c|c|c|c|c|}
\hline \multicolumn{2}{|c|}{ Parameter/range } & \multirow{2}{*}{$\begin{array}{l}\text { MTX } \\
\text { Total Cost }\end{array}$} & \multirow{2}{*}{$\begin{array}{l}\text { Lef+MTX } \\
\text { Total Cost }\end{array}$} & \multirow{2}{*}{$\begin{array}{c}\text { Inf }+\mathbf{M T X} \\
\text { Total Cost }\end{array}$} & \multirow{2}{*}{$\begin{array}{l}\text { Ada+MTX } \\
\text { Total Cost }\end{array}$} & \multirow{2}{*}{$\begin{array}{c}\text { Eta+MTX } \\
\text { Total Cost }\end{array}$} & \multirow{2}{*}{$\begin{array}{l}\text { MTX } \\
\text { QALYs }\end{array}$} & \multirow{2}{*}{$\begin{array}{r}\text { Lef+MTX } \\
\text { QALYs }\end{array}$} & \multirow{2}{*}{$\begin{array}{r}\text { Inf+MTX } \\
\text { QALYs }\end{array}$} & \multirow{2}{*}{$\begin{array}{c}\text { Ada+MTX } \\
\text { QALYs }\end{array}$} & \multirow{2}{*}{$\begin{array}{l}\text { Eta+MTX } \\
\text { QALYs }\end{array}$} \\
\hline & & & & & & & & & & & \\
\hline Base Case & & 82,956 & 116,991 & 189,145 & 187,748 & 314,895 & 12.29 & 12.94 & 12.51 & 13.28 & 15.84 \\
\hline \multicolumn{2}{|c|}{ Adalimumab (\$240) } & 82,054 & 115,798 & 186,126 & 184,532 & 310,690 & 12.17 & 12.83 & 12.40 & 13.17 & 15.74 \\
\hline & $(\$ 468)$ & 82,378 & 116,040 & 188,697 & 187,627 & 318,616 & 12.20 & 12.84 & 12.40 & 13.18 & 15.80 \\
\hline \multirow[t]{2}{*}{ Etanercept } & $(\$ 240)$ & 82,054 & 115,798 & 186,126 & 184,532 & 310,690 & 12.17 & 12.83 & 12.40 & 13.17 & 15.74 \\
\hline & $(\$ 468)$ & 82,378 & 116,040 & 188,697 & 187,627 & 318,616 & 12.20 & 12.84 & 12.40 & 13.18 & 15.80 \\
\hline \multirow[t]{2}{*}{ Infliximab } & $(\$ 240)$ & 82,054 & 115,798 & 186,126 & 184,532 & 310,690 & 12.17 & 12.83 & 12.40 & 13.17 & 15.74 \\
\hline & $(\$ 468)$ & 82,378 & 116,040 & 188,697 & 187,627 & 318,616 & 12.20 & 12.84 & 12.40 & 13.18 & 15.80 \\
\hline \multicolumn{2}{|c|}{ Leflunomide (\$347) } & 81,691 & 113,664 & 186,649 & 184,830 & 311,460 & 12.12 & 12.77 & 12.34 & 13.12 & 15.63 \\
\hline & $(\$ 598)$ & 82,190 & 117,091 & 187,375 & 185,751 & 315,068 & 12.19 & 12.83 & 12.41 & 13.17 & 15.70 \\
\hline \multirow[t]{2}{*}{ MTX } & (\$299) & 81,947 & 115,987 & 187,949 & 185,612 & 315,163 & 12.23 & 12.86 & 12.45 & 13.21 & 15.77 \\
\hline & $(\$ 501)$ & 82,608 & 116,121 & 188,349 & 186,587 & 313,890 & 12.17 & 12.83 & 12.40 & 13.18 & 15.71 \\
\hline
\end{tabular}

MTX: Standard MTX treatment, Lef+MTX: Leflunomide+MTX, Inf+MTX: Infliximab+MTX, Ada+MTX: Adalimumab+MTX, Eta+MTX: Etanercept+MTX 
Table 21. One-way sensitivity analyses - Hospitalization costs

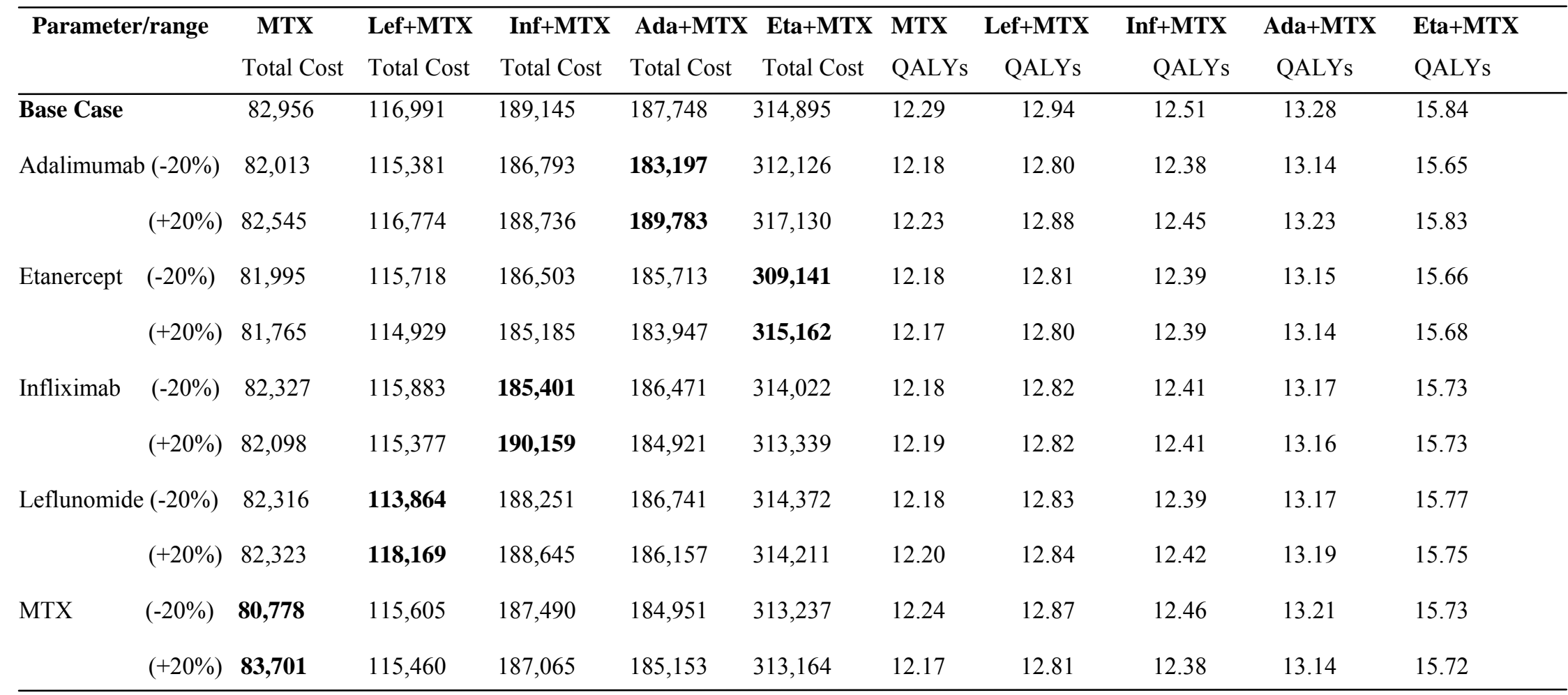

MTX: Standard MTX treatment, Lef+MTX: Leflunomide+MTX, Inf+MTX: Infliximab+MTX, Ada+MTX: Adalimumab+MTX, Eta+MTX: Etanercept+MTX 
Table 22. One-way sensitivity analyses - Costs of treating mild/moderate adverse event, severe adverse event, joint replacement, and palliative care treatment

\begin{tabular}{|c|c|c|c|c|c|c|c|c|c|c|}
\hline \multirow[t]{2}{*}{ Parameter/range } & MTX & Lef+MTX & Inf+MTX & Ada+MTX & Eta+MTX & MTX & Lef+MTX & Inf+MTX & Ada+MTX & Eta + MTX \\
\hline & Total Cost & Total Cost & Total Cost & Total Cost & Total Cost & QALYs & QALYs & QALYs & QALYs & QALYs \\
\hline Base Case & 82,956 & 116,991 & 189,145 & 187,748 & 314,895 & 12.29 & 12.94 & 12.51 & 13.28 & 15.84 \\
\hline \multicolumn{11}{|c|}{ Costs of treating mild/moderate adverse event } \\
\hline$(\$ 126)$ & 82,014 & 115,240 & 186,511 & 185,077 & 316,321 & 12.17 & 12.82 & 12.39 & 13.16 & 15.80 \\
\hline$(\$ 204)$ & 82,640 & 116,400 & 187,147 & 186,751 & 316,475 & 12.24 & 12.88 & 12.43 & 13.22 & 15.83 \\
\hline \multicolumn{11}{|c|}{$\underline{\text { Costs of treating severe adverse event }}$} \\
\hline$(-20 \%)$ & 81,127 & 114,405 & 185,071 & 182,421 & 312,683 & 12.20 & 12.81 & 12.40 & 13.15 & 15.73 \\
\hline$(+20 \%)$ & 83,550 & 117,147 & 189,318 & 188,943 & 315,027 & 12.23 & 12.86 & 12.44 & 13.21 & 15.76 \\
\hline \multicolumn{11}{|c|}{ Costs of joint replacement } \\
\hline$(\$ 15,592)$ & 79,518 & 113,048 & 184,607 & 182,407 & 310,011 & 12.21 & 12.86 & 12.42 & 13.20 & 15.72 \\
\hline$(\$ 23,388)$ & 85,075 & 118,490 & 190,108 & 188,345 & 320,584 & 12.18 & 12.81 & 12.39 & 13.16 & 15.78 \\
\hline \multicolumn{11}{|c|}{ Costs of palliative care treatment } \\
\hline$(\$ 912)$ & 73,013 & 106,675 & 177,380 & 176,135 & 307,692 & 12.24 & 12.85 & 12.43 & 13.19 & 15.79 \\
\hline$(\$ 1,369)$ & 91,585 & 125,270 & 198,056 & 195,972 & 324,216 & 12.20 & 12.86 & 12.42 & 13.21 & 15.82 \\
\hline
\end{tabular}

MTX: Standard MTX treatment, Lef+MTX: Leflunomide+MTX, Inf+MTX: Infliximab+MTX, Ada+MTX: Adalimumab+MTX, Eta+MTX: Etanercept+MTX 
Table 23. Summary of main results in one-way sensitivity analyses

\begin{tabular}{|c|c|c|c|c|c|}
\hline Parameter/range & & Leflunomide+MTX & Infliximab+MTX & Adalimumab+MTX & Etanercept+MTX \\
\hline \multicolumn{2}{|l|}{ Base Case } & 52,833 & Dominated & Extended Domination & 49,724 \\
\hline \multicolumn{6}{|c|}{ Probability of Achieving ACR 20 Response } \\
\hline All Five Treatments & $(-20 \%)$ & 52,058 & Dominated & Extended Domination & 38,033 \\
\hline \multirow[t]{2}{*}{ Adalimumab } & $(-20 \%)$ & 52,058 & Dominated & Extended Domination & 46,773 \\
\hline & $(+20 \%)$ & 52,058 & Dominated & Extended Domination & 38,033 \\
\hline \multirow[t]{2}{*}{ Etanercept } & $(-20 \%)$ & 52,058 & Dominated & Extended Domination & 40,078 \\
\hline & $(+20 \%)$ & 52,058 & Dominated & Extended Domination & 34,986 \\
\hline \multirow[t]{2}{*}{ Leflunomide } & $(-20 \%)$ & 45,638 & Dominated & Extended Domination & 38,033 \\
\hline & $(+20 \%)$ & 63,126 & Dominated & Extended Domination & 38,033 \\
\hline \multirow[t]{2}{*}{ MTX } & $(-20 \%)$ & Extended Domination & Dominated & Extended Domination & 38,033 \\
\hline & $(+20 \%)$ & 45,256 & Dominated & Extended Domination & 38,033 \\
\hline
\end{tabular}

All treatments compared to Standard MTX treatment 
Table 23. Summary of main results in one-way sensitivity analyses

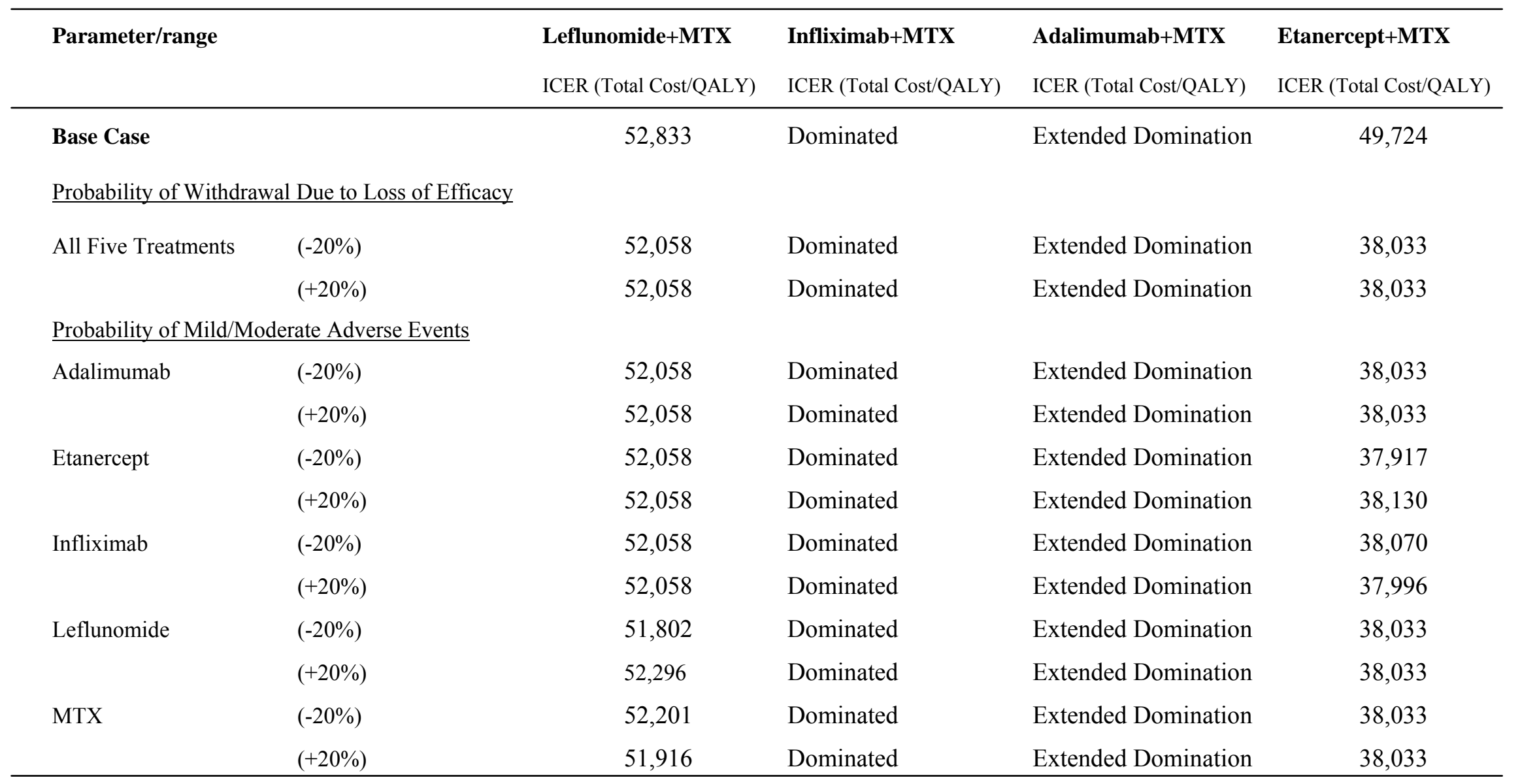

All treatments compared to Standard MTX treatment 
Table 23. Summary of main results in one-way sensitivity analyses

\begin{tabular}{|c|c|c|c|c|c|}
\hline \multirow[t]{2}{*}{ Parameter/range } & & Leflunomide+MTX & Infliximab+MTX & Adalimumab+MTX & Etanercept+MTX \\
\hline & & ICER (Total Cost/QALY) & ICER (Total Cost/QALY) & ICER (Total Cost/QALY) & ICER (Total Cost/QALY) \\
\hline Base Case & & 52,833 & Dominated & Extended Domination & 49,724 \\
\hline \multicolumn{6}{|c|}{ Probability of Joint Replacement } \\
\hline & $(0.1)$ & 52,069 & Dominated & Extended Domination & 38,193 \\
\hline & $(0.5)$ & 52,058 & Dominated & Extended Domination & 37,966 \\
\hline \multicolumn{6}{|l|}{ Discount Rate } \\
\hline & $(0 \%)$ & 51,253 & Dominated & Extended Domination & 38,923 \\
\hline & $(5 \%)$ & 52,493 & Dominated & Extended Domination & 37,017 \\
\hline
\end{tabular}

All treatments compared to the reference case: Standard MTX treatment 
Table 23. Summary of main results in one-way sensitivity analyses

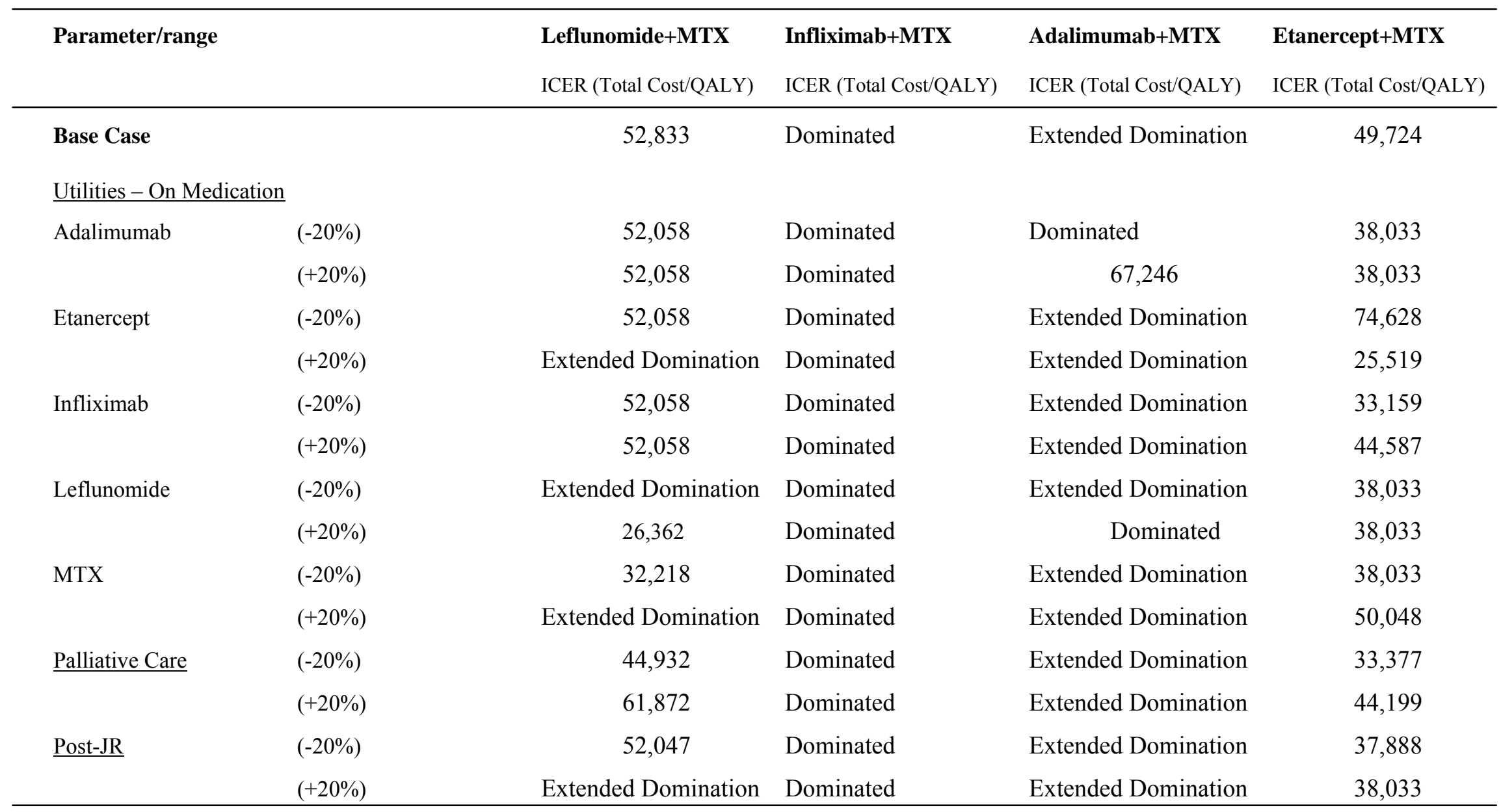

All treatments compared to the reference case: Standard MTX treatment

Post-JR $=$ Post-Joint Replacement 
Table 23. Summary of main results in one-way sensitivity analyses

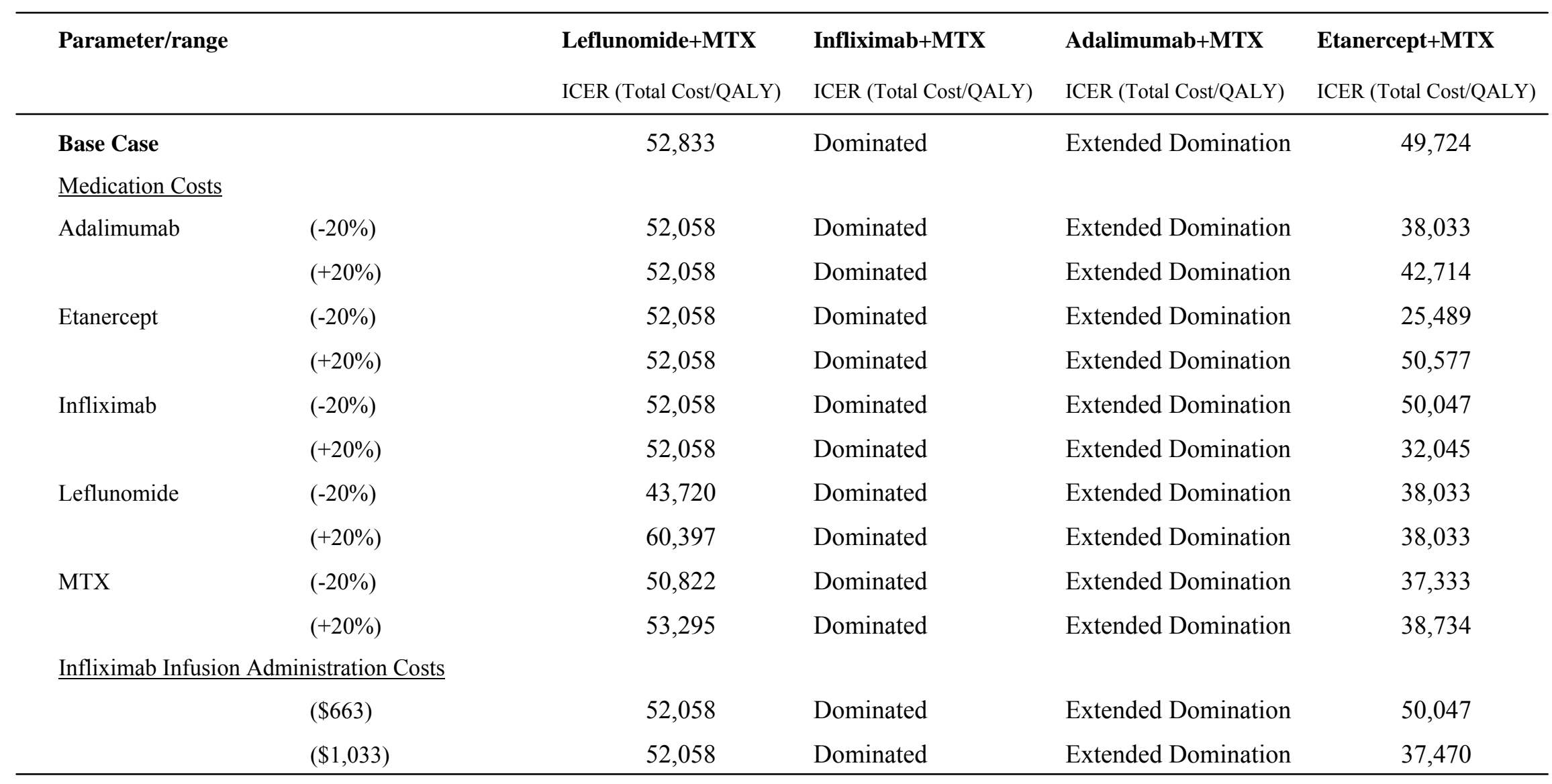

All treatments compared to the reference case: Standard MTX treatment 
Table 23. Summary of main results in one-way sensitivity analyses

\begin{tabular}{|c|c|c|c|c|c|}
\hline \multicolumn{2}{|l|}{ Parameter/range } & $\begin{array}{l}\text { Leflunomide+MTX } \\
\text { ICER (Total Cost/QALY) }\end{array}$ & $\begin{array}{l}\text { Infliximab+MTX } \\
\text { ICER (Total Cost/QALY) }\end{array}$ & $\begin{array}{l}\text { Adalimumab+MTX } \\
\text { ICER (Total Cost/QALY) }\end{array}$ & $\begin{array}{l}\text { Etanercept+MTX } \\
\text { ICER (Total Cost/QALY) }\end{array}$ \\
\hline \multicolumn{6}{|c|}{ Monitoring Costs in First Six Months } \\
\hline \multirow{2}{*}{ TNF inhibitors } & $(\$ 730)$ & 52,058 & Dominated & Extended Domination & 38,033 \\
\hline & $(\$ 1,262)$ & 52,058 & Dominated & Extended Domination & 38,033 \\
\hline Leflunomide & $(\$ 852)$ & 52,199 & Dominated & Extended Domination & 38,033 \\
\hline \multirow[t]{2}{*}{ MTX } & $(\$ 488)$ & 52,228 & Dominated & Extended Domination & 38,033 \\
\hline & $(\$ 927)$ & 51,889 & Dominated & Extended Domination & 38,033 \\
\hline \multicolumn{6}{|c|}{ Monitoring Costs after Six Months } \\
\hline Leflunomide & $(\$ 598)$ & 52,199 & Dominated & Extended Domination & 38,033 \\
\hline \multirow[t]{2}{*}{ MTX } & $(\$ 299)$ & 53,022 & Dominated & Extended Domination & 38,033 \\
\hline & $(\$ 501)$ & 51,168 & Dominated & Extended Domination & 38,033 \\
\hline
\end{tabular}

All treatments compared to the reference case: Standard MTX treatment 
Table 23. Summary of main results in one-way sensitivity analyses

\begin{tabular}{|c|c|c|c|c|c|}
\hline \multicolumn{2}{|l|}{ Parameter/range } & \multirow{2}{*}{$\begin{array}{l}\text { Leflunomide+MTX } \\
\text { ICER (Total Cost/QALY) }\end{array}$} & \multirow{2}{*}{$\begin{array}{l}\text { Infliximab+MTX } \\
\text { ICER (Total Cost/QALY) }\end{array}$} & \multirow{2}{*}{$\begin{array}{l}\text { Adalimumab+MTX } \\
\text { ICER (Total Cost/QALY) }\end{array}$} & \multirow{2}{*}{$\begin{array}{l}\text { Etanercept+MTX } \\
\text { ICER (Total Cost/QALY) }\end{array}$} \\
\hline & & & & & \\
\hline Base Case & & 52,833 & Dominated & Extended Domination & 49,724 \\
\hline \multicolumn{6}{|c|}{ Costs of Hospitalization } \\
\hline \multirow[t]{2}{*}{ Adalimumab } & $(-20 \%)$ & 52,058 & Dominated & Extended Domination & 38,033 \\
\hline & $(+20 \%)$ & 52,058 & Dominated & Extended Domination & 49,248 \\
\hline Etanercept & $(+20 \%)$ & 52,058 & Dominated & Extended Domination & 39,244 \\
\hline \multirow[t]{2}{*}{ Infliximab } & $(-20 \%)$ & 52,058 & Dominated & Extended Domination & 50,047 \\
\hline & $(+20 \%)$ & 52,058 & Dominated & Extended Domination & 37,035 \\
\hline Leflunomide & $(-20 \%)$ & 48,603 & Dominated & Extended Domination & 38,033 \\
\hline \multicolumn{6}{|c|}{ Cost of Treating Mild/ Moderate Adverse Events } \\
\hline & $(\$ 126)$ & 51,924 & Dominated & Extended Domination & 37,938 \\
\hline & $(\$ 204)$ & 52,193 & Dominated & Extended Domination & 38,129 \\
\hline
\end{tabular}

All treatments compared to the reference case: Standard MTX treatment 
Table 23. Summary of main results in one-way sensitivity analyses

\begin{tabular}{|c|c|c|c|c|c|}
\hline \multirow[t]{2}{*}{ Parameter/range } & & Leflunomide+MTX & Infliximab+MTX & Adalimumab+MTX & Etanercept+MTX \\
\hline & & ICER (Total Cost/QALY) & ICER (Total Cost/QALY) & ICER (Total Cost/QALY) & ICER (Total Cost/QALY) \\
\hline Base Case & & 52,833 & Dominated & Extended Domination & 49,724 \\
\hline \multicolumn{6}{|c|}{$\underline{\text { Cost of Treating Severe Adverse Events }}$} \\
\hline & $(-20 \%)$ & 52,492 & Dominated & Extended Domination & 37,993 \\
\hline & $(+20 \%)$ & 51,625 & Dominated & Extended Domination & 38,073 \\
\hline \multicolumn{6}{|c|}{$\underline{\text { Cost of Joint Replacement }}$} \\
\hline & $(\$ 15,592)$ & 52,060 & Dominated & Extended Domination & 38,058 \\
\hline & $(\$ 23,388)$ & 52,057 & Dominated & Extended Domination & 38,008 \\
\hline \multicolumn{6}{|c|}{ Costs of Palliative Care Treatment } \\
\hline & $(\$ 912)$ & 52,698 & Dominated & Extended Domination & 38,623 \\
\hline & $(\$ 1,369)$ & 51,418 & Dominated & Extended Domination & 37,443 \\
\hline
\end{tabular}

All treatments compared to the reference case: Standard MTX treatment 
The total costs were sensitive to changes in the medication costs. However, the dominance status of each considered treatments remained the same as in the base-case analysis. As seen in the results, changes in the cost parameters influenced only the total costs and not the effectiveness and resulted in marked changes in the ICERs for the treatments. For example, reducing the medication costs of etanercept and leflunomide (the two nondominated treatments) by $20 \%$ resulted in an ICER of $\$ 25,489 /$ QALY and $\$ 43,720 / \mathrm{QALY}$, respectively. Conversely, increasing the medication costs of etanercept and leflunomide by $20 \%$ resulted in an ICER of $\$ 50,577 /$ QALY and \$60,397/QALY, respectively.

Changes in the utility parameter also resulted in changes in the ICERs. Increasing the utilities of etanercept and leflunomide by $20 \%$ resulted in an ICER of $\$ 25,519 / \mathrm{QALY}$ and $\$ 26,362 / \mathrm{QALY}$, respectively. Decreasing the utilities of these treatments by $20 \%$ resulted in an ICER of $\$ 74,628 /$ QALY for etanercept and leflunomide was ruled out by extended dominance. Results varied little with changes in the utilities associated with palliative care and post-joint replacement care.

The results were most sensitive to changes in medication costs, cost of palliative care, and probability of total withdrawals (Figure 5 and 6). The results were not sensitive to the changes in the probability of ACR 20 responses and probability of lack of efficacy of treatments. Also, results varied little with the changes in probability of joint replacement, discount rate, monitoring costs, costs of hospitalizations, and costs of severe and mild/moderate adverse events. The ICER for leflunomide plus MTX 
Figure 5. One-way sensitivity analysis: Etanercept

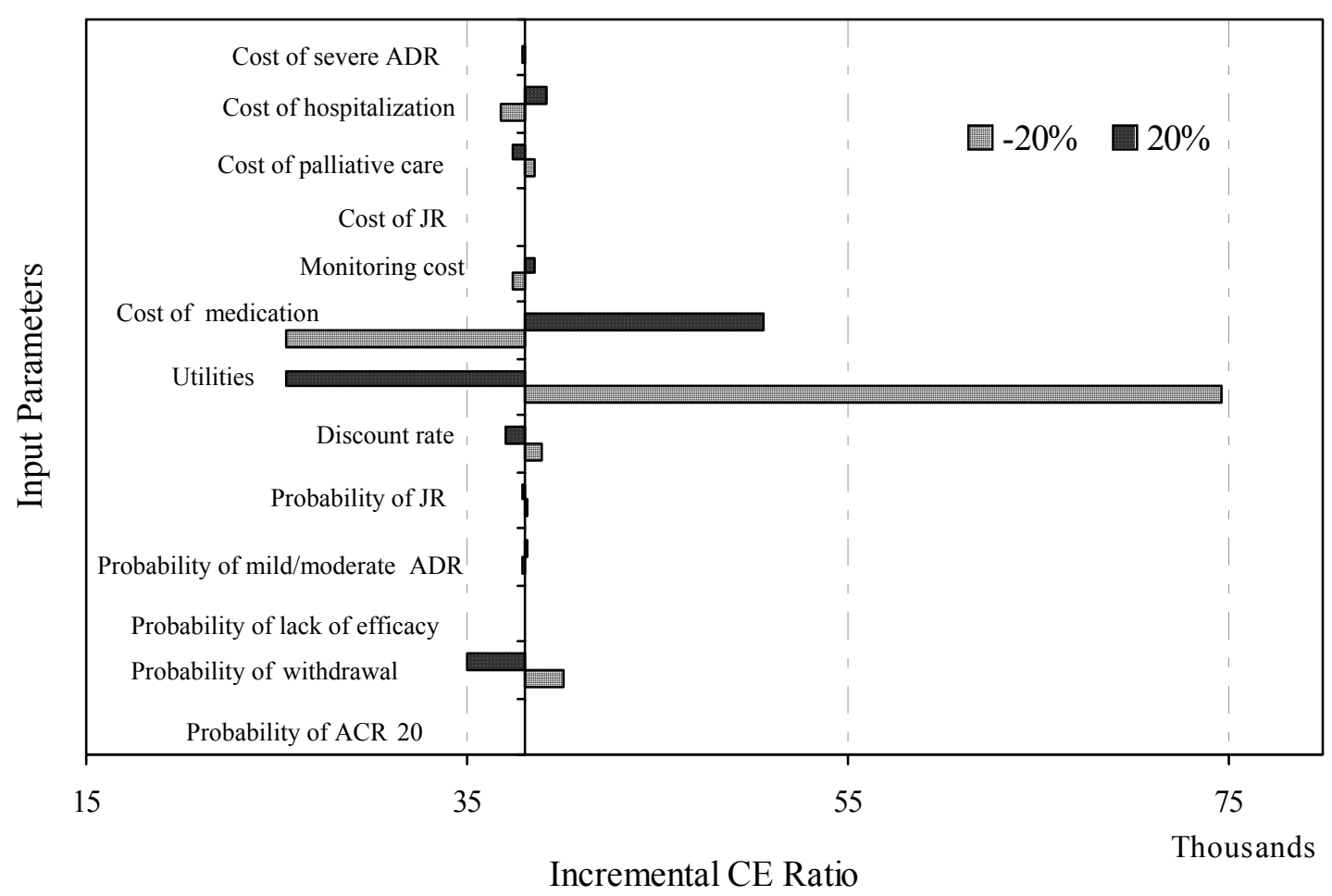

$\mathrm{ADR}=$ Adverse Drug Reaction

$\mathrm{ACR}=$ American College of Rheumatology

$\mathrm{JR}=$ Joint Replacement 
Figure 6. One-way sensitivity analysis: Leflunomide

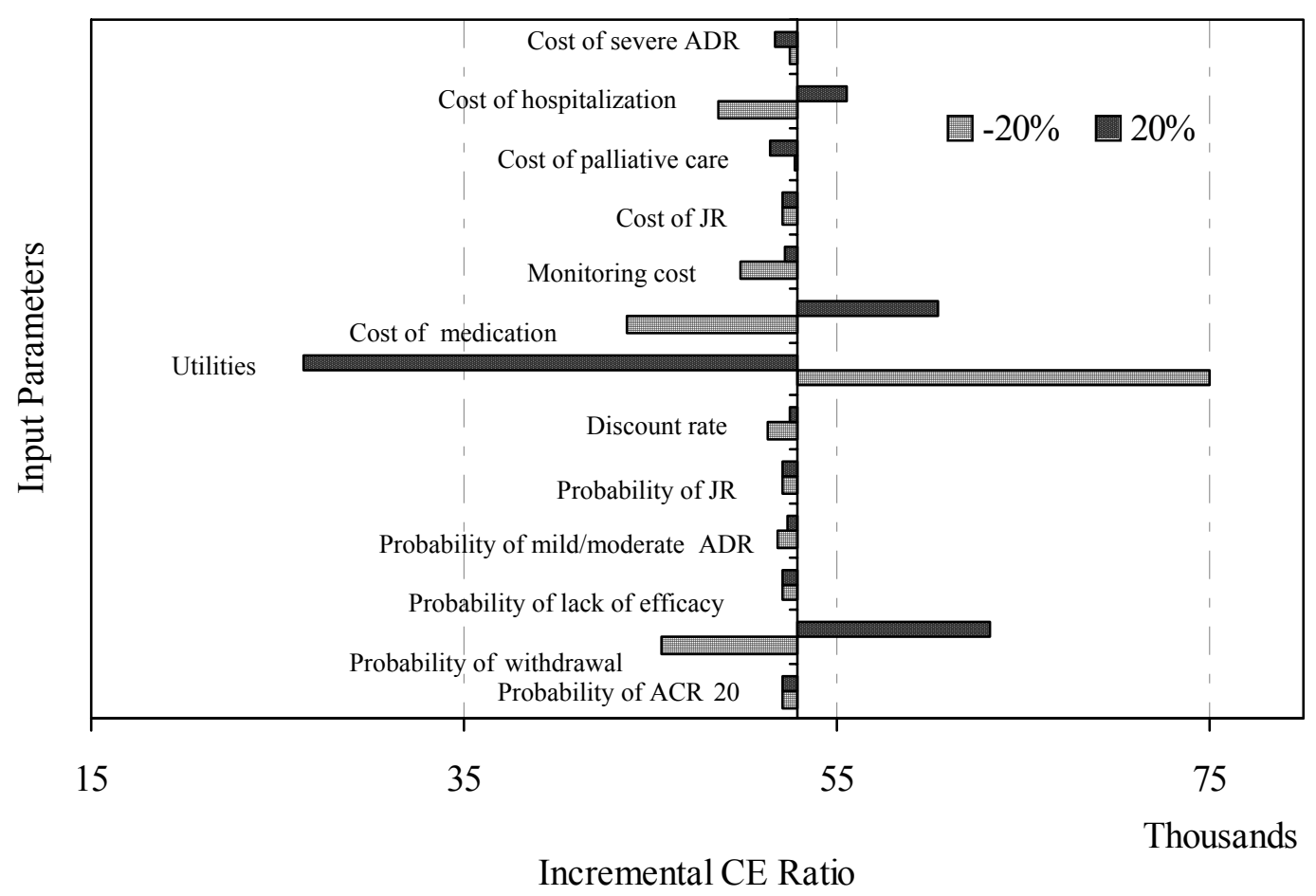

$\mathrm{ADR}=$ Adverse Drug Reaction

$\mathrm{ACR}=$ American College of Rheumatology

$\mathrm{JR}=\mathrm{Joint}$ Replacement 
remained fairly stable across the ranges of all variables, except when medication costs and utilities of leflunomide were reduced or increased by $20 \%$.

The ICER for etanercept plus MTX was found to be even more favorable in the sensitivity results than in the base-case analysis. The ICER for etanercept plus MTX in the sensitivity analysis for a number of variables was around $\$ 38,000 / \mathrm{QALY}$ compared to an ICER of $\$ 49,724 / \mathrm{QALY}$ reported in the base-case analysis. This is because the total costs of adalimumab plus MTX and infliximab plus MTX are very similar to each other. Varying any of the variables in the sensitivity analysis between $+20 \%$ resulted in a change in position of adalimumab plus MTX and infliximab plus MTX in the calculation of incremental costs and this made the ICER for etanercept plus MTX even more favorable. For example, when the treatment cost of adalimumab plus MTX is reduced by $20 \%$, the lifetime cost of adalimumab plus MTX is $\$ 167,384$ while that of infliximab plus MTX is $\$ 187,189$ and etanercept plus MTX is $\$ 316,082$. The incremental cost of etanercept plus MTX is calculated by subtracting infliximab plus MTX cost from etanercept plus MTX cost. However, when treatment cost of adalimumab plus MTX is increased by $20 \%$, the lifetime cost of adalimumab plus MTX increases to $\$ 206,667$ while that of infliximab plus MTX and etanercept plus MTX remain approximately the same. The incremental cost of etanercept plus MTX is now calculated from adalimumab plus MTX since the cost of adalimumab plus MTX is higher than that of infliximab plus MTX. This results in a lower ICER for etanercept plus MTX treatment. 


\section{Probabilistic Sensitivity Analysis}

Because the overall variability of a model is poorly characterized by a univariate sensitivity analysis, probabilistic sensitivity analysis (second order Monte Carlo simulation) was also used to characterize this variability. As discussed earlier, each parameter in the model, instead of taking a single value, was assumed to be a variable quantity with a known range of possible values and an associated distribution function. Since all costs, probabilities, and utilities were given ranges, a triangular distribution was specified for each of the variable. Triangular distribution takes into consideration the lowest, highest, and the likeliest value of any variable.

The process of resampling from each of the distributions and recalculating the cost-effectiveness from the model was repeated 10,000 times to generate the total costs, effectiveness, and ICERs of each treatment. The results are presented in Table 24 and are similar to those obtained in the base-case analysis. Etanercept plus MTX and leflunomide plus MTX had an ICER of \$49,689/QALY and \$51,288/QALY, respectively. Infliximab plus MTX was dominated by leflunomide plus MTX and adalimumab plus MTX was ruled out by extended domination. The advantage of using a probabilistic sensitivity analysis is that it instills more confidence in the validity of the model by taking into consideration the relevant uncertainty in the parameter; and by specifying a prior distribution for a particular parameter that increases the clarity of the model. 
Table 24. Probabilistic sensitivity analysis results for total costs, effectiveness, and incremental cost-effectiveness ratios of different treatments for patients with RA that inadequately respond to MTX (using age-, sex-, race-adjusted all-cause mortality rate

\begin{tabular}{llll}
\hline Treatments & $\begin{array}{l}\text { Total costs } \\
\mathbf{( \$ )}\end{array}$ & $\begin{array}{l}\text { Effectiveness } \\
\text { (QALYs) }\end{array}$ & $\begin{array}{l}\text { Incremental } \\
\text { cost-effectiveness ratios } \\
\text { (\$/QALY) }\end{array}$ \\
\hline Standard MTX & 83,003 & 12.25 & - \\
Leflunomide+MTX & 116,506 & 12.91 & 51,288 \\
Infliximab+MTX & 186,868 & 12.47 & Dominated $^{\#}$ \\
Adalimumab+MTX & 187,352 & 13.25 & Extended Domination $^{\text {\#\# }}$ \\
Etanercept+MTX & 316,468 & 15.85 & 49,689 \\
\hline
\end{tabular}

All treatments compared to the reference case: Standard MTX treatment

MTX=methotrexate

${ }^{*}$ Total costs $=$ Direct costs + Indirect costs (Indirect costs calculated as same as direct costs)

\# Infliximab+MTX dominated by Leflunomide+MTX

\#\# Adalimumab+MTX dominated by a blend of Leflunomide+MTX and Etanercept+MTX with a coefficient of inequity between 0.645 and 0.884 . 


\section{Effect of RA-adjusted Mortality Rate on ICERs}

There are uncertainties relating to the available data on the effects of RA on the risk of mortality. To examine the effect of increased risk of mortality due to RA, it was assumed that mortality was two-fold greater compared with an age-, sex-, and racematched general population. Results of the analyses comparing the direct costs, total costs, effectiveness, and ICERs of the five treatments are shown in Tables 25 and 26.

The dominance status of each treatment remained the same as in the base-case analysis using total costs and direct costs. The standard MTX treatment was the least expensive $($ direct $\operatorname{costs}=\$ 62,504$, total costs $=\$ 71,867)$ and lowest QALY gain $(10.9$ QALY). Etanercept plus MTX was the most expensive treatment (direct costs $=$ $\$ 177,197$, total costs $=\$ 297,171)$ and highest QALY gain $(14.3$ QALY). This treatment provided more health benefits and was more costly than standard MTX treatment, with resulting cost-effectiveness ratios of \$24,421/QALY (direct costs) and \$50,782/QALY (total costs). The second most cost-effective option was leflunomide plus MTX with an ICER of \$28,127/QALY (direct costs) and \$51,905/QALY (total costs). Infliximab plus MTX was dominated by adalimumab plus MTX and adalimumab plus MTX was ruled out through extended dominance. 
Table 25. Results for total costs and effectiveness using Monte Carlo simulations of 10,000 hypothetical patients with RA that inadequately respond to MTX (using RA-adjusted mortality rates)

\begin{tabular}{|c|c|c|c|c|c|c|c|c|}
\hline \multirow[b]{2}{*}{ Treatment Strategy } & \multicolumn{4}{|c|}{ Total Costs* (\$) } & \multicolumn{4}{|c|}{ Effectiveness (QALYs) } \\
\hline & Mean $( \pm S D) \$$ & $\operatorname{Min} \$$ & Median \$ & Max \$ & Mean $( \pm S D)$ & Min & Median & Max \\
\hline Standard MTX & $71,867( \pm 28,069)$ & 1,362 & 77,196 & 153,563 & $10.91( \pm 3.40)$ & 0.22 & 12.00 & 18.61 \\
\hline Leflunomide+MTX & $104,865 \quad( \pm 53,091)$ & 2,276 & 97,161 & 343,265 & $11.55( \pm 3.65)$ & 0.25 & 12.46 & 23.46 \\
\hline Infliximab+MTX & $173,920( \pm 122,090)$ & 6,360 & 135,807 & 858,419 & $11.13( \pm 3.47)$ & 0.23 & 12.16 & 21.08 \\
\hline Adalimumab+MTX & $173,314( \pm 112,921)$ & 5,233 & 142,950 & 731,753 & $11.88( \pm 3.81)$ & 0.28 & 12.68 & 25.85 \\
\hline Etanercept+MTX & $297,171( \pm 190,522)$ & 5,184 & 256,584 & 851,614 & $14.32( \pm 5.22)$ & 0.29 & 14.29 & 33.88 \\
\hline
\end{tabular}

All treatments compared to the reference case: Standard MTX treatment

MTX=methotrexate

${ }^{*}$ Total costs $=$ Direct costs + Indirect costs (Indirect costs calculated the same as direct costs)

QALYs = Quality adjusted life years

MTX = Methotrexate

$\mathrm{SD}=$ Standard deviation

Min $=$ Minimum

Max=Maximum 
Table 26. Direct costs, effectiveness, total costs, and cost-effectiveness of different treatments for patients with RA that inadequately respond to MTX (using RA-adjusted mortality rates)

\begin{tabular}{|c|c|c|c|c|c|c|}
\hline Standard MTX & 62,504 & 10.90 & - & 71,867 & 10.91 & - \\
\hline Infliximab+MTX & 118,205 & 11.14 & Dominated $^{+}$ & 173,920 & 11.13 & Dominated $^{\#}$ \\
\hline Adalimumab+MTX & 118,383 & 11.87 & Extended Domination ${ }^{++}$ & 173,314 & 11.88 & Extended Domination \\
\hline Etanercept+MTX & 177,197 & 14.28 & 24,421 & 297,171 & 14.32 & 50,782 \\
\hline
\end{tabular}

All treatments compared to the reference case: Standard MTX treatment

MTX=methotrexate

${ }^{*}$ Total costs $=$ Direct costs + Indirect costs (Indirect costs calculated as same as direct costs)

${ }^{+}$Infliximab+MTX dominated by Adalimumab+MTX

${ }^{++}$Adalimumab+MTX dominated by a blend of Leflunomide+MTX and Etanercept+MTX with a coefficient of inequity between 0.609 and 0.878 .

\# Infliximab+MTX dominated by Adalimumab+MTX

\#\# Adalimumab+MTX dominated by a blend of Leflunomide+MTX and Etanercept+MTX with a coefficient of inequity between 0.644 and 0.880 . 


\section{Effect of Indirect Costs on ICERs}

Additional analyses were conducted to determine if there was any change in the dominance of treatments with regards to changes in the indirect costs. Indirect costs were calculated as two and three times that of direct costs. Tables 27 and 28 give the total costs, effectiveness, and ICERs for each treatment using both age-, sex-, and raceadjusted mortality rate and RA-adjusted mortality rates.

When indirect costs were considered as twice that of direct costs, etanercept plus MTX had the lowest ICER of $\$ 75,502$ to $\$ 75,577 /$ QALY followed closely by leflunomide plus MTX with an ICER of \$75,688 to \$77,398/QALY. However, when indirect costs were considered as three times that of direct costs, leflunomide plus MTX had the lowest ICER of $\$ 101,704$ to $\$ 101,919 /$ QALY followed by etanercept plus MTX by with an ICER of $\$ 101,875$ to $\$ 102,548$ /QALY. In both scenarios, infliximab plus MTX and adalimumab plus MTX were ruled out by dominance and extended dominance, respectively. 
Table 27. Total costs, effectiveness, and incremental cost-effectiveness ratios of different treatments for patients with RA that inadequately respond to MTX

\begin{tabular}{|c|c|c|c|c|c|c|}
\hline Treatment Strategy & $\begin{array}{l}\text { Total costs* } \\
\text { (\$) }\end{array}$ & $\begin{array}{l}\text { Effectiveness } \\
\text { (QALYs) }\end{array}$ & $\begin{array}{l}\text { Incremental } \\
\text { cost-effectiveness } \\
\text { ratios } \\
\text { (\$/QALY) } \\
\end{array}$ & $\begin{array}{l}\text { Total costs } \\
\text { (\$) }\end{array}$ & $\begin{array}{l}\text { Effectiveness } \\
\text { (QALYs) }\end{array}$ & $\begin{array}{l}\text { Incremental } \\
\text { cost-effectiveness } \\
\text { ratios } \\
\text { (\$/QALY) } \\
\end{array}$ \\
\hline Standard MTX & 92,428 & 12.25 & - & 81,565 & 10.93 & - \\
\hline Infliximab+MTX & 246,826 & 12.47 & Dominated $^{\#}$ & 230,437 & 11.15 & Dominated $^{\S}$ \\
\hline Adalimumab+MTX & 243,493 & 13.25 & Extended Domination & 234,753 & 11.89 & Extended Domination $^{\S \S}$ \\
\hline Etanercept + MTX & 440,085 & 15.86 & 75,502 & 418,168 & 14.38 & 75,577 \\
\hline
\end{tabular}

All treatments compared to the reference case: Standard MTX treatment

MTX=methotrexate

ASR $=$ Age, Sex, Race

* Total costs $=$ Direct costs + Indirect costs (Indirect costs calculated as two times that of direct costs)

\# Infliximab+MTX dominated by Adalimumab +MTX

\#Adalimumab+MTX dominated by a blend of Leflunomide+MTX and Etanercept+MTX with a coefficient of inequity between 0.659 and 0.882 .

$\S$ Infliximab+MTX dominated by Adalimumab +MTX

$\$$ Adalimumab+MTX dominated by a blend of Leflunomide+MTX and Etanercept+MTX with a coefficient of inequity between 0.652 and 0.879 . 
Table 28. Total costs, effectiveness, and incremental cost-effectiveness ratios of different strategies for patients with RA that inadequately respond to MTX

\begin{tabular}{|c|c|c|c|c|c|c|}
\hline \multirow[b]{2}{*}{ Treatment Strategy } & \multicolumn{3}{|c|}{ Normal ASR-adjusted All-cause Mortality Rates } & \multicolumn{3}{|c|}{ RA Adjusted All-cause Mortality Rates } \\
\hline & $\begin{array}{l}\text { Total costs } \\
\text { (\$) }\end{array}$ & $\begin{array}{l}\text { Effectiveness } \\
\text { (QALYs) }\end{array}$ & $\begin{array}{l}\text { Incremental } \\
\text { cost-effectiveness } \\
\text { ratios } \\
\text { (\$/QALY) }\end{array}$ & $\begin{array}{l}\text { Total costs } \\
\text { (\$) }\end{array}$ & $\begin{array}{l}\text { Effectiveness } \\
\text { (QALYs) }\end{array}$ & $\begin{array}{l}\text { Incremental } \\
\text { cost-effectiveness } \\
\text { ratios } \\
\text { (\$/QALY) }\end{array}$ \\
\hline Standard MTX & 102,224 & 12.30 & - & 90,509 & 10.87 & - \\
\hline Leflunomide+MTX & 165,852 & 12.93 & 101,704 & 152,332 & 11.48 & 101,919 \\
\hline Infliximab+MTX & 305,123 & 12.52 & Dominated $^{\#}$ & 290,420 & 11.09 & Dominated $^{\S}$ \\
\hline Adalimumab + MTX & 297,229 & 13.27 & Extended Domination ${ }^{\# \#}$ & 280,414 & 11.81 & Extended Domination ${ }^{\S}$ \\
\hline Etanercept+MTX & 557,684 & 15.82 & 101,875 & 530,070 & 14.25 & 102,548 \\
\hline
\end{tabular}

All treatments compared to the reference case: Standard MTX treatment

MTX=methotrexate

ASR $=$ Age, Sex, Race

${ }^{*}$ Total costs $=$ Direct costs + Indirect costs (Indirect costs calculated as three times that of direct costs)

\# Infliximab+MTX dominated by Adalimumab +MTX

\#Adalimumab+MTX dominated by a blend of Leflunomide+MTX and Etanercept+MTX with a coefficient of inequity between 0.665 and 0.883 .

$\S$ Infliximab+MTX dominated by Adalimumab +MTX

$\$ \S$ Adalimumab+MTX dominated by a blend of Leflunomide+MTX and Etanercept+MTX with a coefficient of inequity between 0.661 and 0.880 . 


\section{Hospitalization Costs}

There are uncertainties relating to the available data on the effects of TNF inhibitors, leflunomide, or methotrexate on hospitalization costs. Unlike the base-case analysis that included hospitalization costs, this analysis was carried out by excluding the hospitalization costs and studying its effect on the ICERs. The results of the analysis using age-, sex-, race-adjusted all-cause mortality rates and RA-adjusted mortality are shown in Table 29. Etanercept plus MTX had the lowest ICER followed by leflunomide plus MTX. Infliximab plus MTX and adalimumab plus MTX were ruled out by dominance and extended dominance, respectively. The ICERs reported in Table 29 are similar to those obtained in the base-case analysis. 
Table 29. Total costs, effectiveness, and cost-effectiveness of different strategies for patients with RA that inadequately respond to MTX (without hospitalization costs)

\begin{tabular}{|c|c|c|c|c|c|c|}
\hline \multirow[b]{2}{*}{ Treatment Strategy } & \multicolumn{3}{|c|}{ ASR-adjusted All-cause Mortality Rates } & \multicolumn{3}{|c|}{ RA Adjusted All-cause Mortality Rates } \\
\hline & $\begin{array}{l}\text { Total costs } \\
\text { (\$) }\end{array}$ & $\begin{array}{l}\text { Effectiveness } \\
\text { (QALYs) }\end{array}$ & $\begin{array}{l}\text { Incremental } \\
\text { cost-effectiveness } \\
\text { ratios } \\
\text { (\$/QALY) }\end{array}$ & $\begin{array}{l}\text { Total costs } \\
\text { (\$) }\end{array}$ & $\begin{array}{l}\text { Effectiveness } \\
\text { (QALYs) }\end{array}$ & $\begin{array}{l}\text { Incremental } \\
\text { cost-effectiveness } \\
\text { ratios } \\
\text { (\$/QALY) }\end{array}$ \\
\hline Standard MTX & 74,487 & 12.29 & - & 63,207 & 10.86 & - \\
\hline Leflunomide+MTX & 105,296 & 12.91 & 50,246 & 93,788 & 11.49 & 48,013 \\
\hline Infliximab+MTX & 171,508 & 12.49 & Dominated $^{\#}$ & 159,098 & 11.07 & Dominated $^{\S}$ \\
\hline Adalimumab+MTX & 176,335 & 13.25 & Extended Domination ${ }^{\# \#}$ & 163,854 & 11.83 & Extended Domination ${ }^{\S \S}$ \\
\hline Etanercept + MTX & 295,922 & 15.82 & 46,529 & 274,493 & 14.20 & 46,714 \\
\hline
\end{tabular}

All treatments compared to the reference case: Standard MTX treatment

MTX $=$ methotrexate

ASR $=$ Age, Sex, Race

${ }^{*}$ Total costs $=$ Direct costs + Indirect costs (Indirect costs calculated as same as direct costs)

\# Infliximab+MTX dominated by Leflunomide+MTX

\#\#Adalimumab+MTX dominated by a blend of Leflunomide+MTX and Etanercept+MTX with a coefficient of inequity between 0.627 and 0.882 .

$\S$ Infliximab+MTX dominated by Leflunomide+MTX

$\S \S$ Adalimumab+MTX dominated by a blend of Leflunomide+MTX and Etanercept+MTX with a coefficient of inequity between 0.612 and 0.874 . 


\section{Effect of Joint Replacement Therapy}

The Markov model included two tracker variables that provided information regarding the proportion of people undergoing joint replacement therapy and the mean age at which people underwent joint replacement therapy. As seen from the results presented in Table 30, the proportion of patients undergoing joint replacement therapy is the lowest for the combination of MTX and TNF inhibitors followed by standard MTX treatment and leflunomide plus MTX. Among the TNF inhibitors, the lowest proportion of patients undergoing joint replacement therapy was seen with etanercept plus MTX. Also, the mean age (68.58 years) and maximum age of undergoing joint replacement (83.5 years) is the highest for etanercept plus MTX indicating that more patients stay on the treatment before moving on to palliative care where they may undergo joint replacement. Thus, patients continue to stay on etanercept plus MTX for a longer time period compared with the other treatments thereby accumulating higher costs and outcomes. 
Table 30. Proportion of patients undergoing joint replacement and age at which joint replacement occurs for each treatment

\begin{tabular}{lclll}
\hline Treatment Strategy & $\begin{array}{l}\text { Proportion of } \\
\text { Patients with JR }\end{array}$ & $\begin{array}{l}\text { Mean Age } \\
\text { in yrs (士SD) }\end{array}$ & $\begin{array}{l}\text { Min. Age } \\
\text { in yrs }\end{array}$ & $\begin{array}{l}\text { Max. Age } \\
\text { in yrs }\end{array}$ \\
\hline Standard MTX & 78.9 & $68.42( \pm 1.7)$ & 67.0 & 79.5 \\
Leflunomide+MTX & 79.5 & $68.33( \pm 1.5)$ & 67.0 & 77.5 \\
Infliximab+MTX & 77.9 & $68.26( \pm 1.5)$ & 67.0 & 78.0 \\
Adalimumab+MTX & 77.9 & $68.40( \pm 1.6)$ & 67.0 & 75.5 \\
Etanercept+MTX & 76.4 & $68.58( \pm 2.0)$ & 67.0 & 83.5
\end{tabular}

Min. Age - Minimum age when patients underwent Joint replacement, Max. Age - Maximum age when patients underwent Joint replacement, SD - Standard deviation, JR - Joint Replacement 


\section{Discussion for Phase I Results}

\section{Background}

Cost-effectiveness analysis has become the standard metric for the assessment of the clinical and economic value of new treatments (Ruff, 1999). These analyses translate the clinical benefits of any treatment into both lifetime costs and effectiveness measures such as quality-adjusted life years (QALYs). Thus, by applying a long time horizon, these analyses account for future benefits and savings or expense. Also, these analyses help decision makers, clinicians, and patients compare relative costeffectiveness of alternative treatments (Wong, 2004).

It has become a practice to compare new treatments to standard care so that new treatments that are more effective and less costly over a lifetime can be cost saving and also dominate the current standard treatments (Gabriel, Tugwell, O’Brien et al., 1999). More often, new treatments introduced in the market are more effective but also cost more. To establish their effectiveness against standard treatments, incremental costeffectiveness analysis are conducted which takes into account the increased cost of the new treatment divided by the increased benefits the new treatment offers. Typically, the outcome measures used in these analyses are expressed as the net additional costs to increase life expectancy by one discounted QALY.

\section{Tumor Necrosis Factor Inhibitors}

Tumor necrosis factor inhibitors offer important treatment options to patients who fail multiple traditional DMARDs. Results from RCTs conducted in patients that 
inadequately respond to MTX alone have shown that these new treatments have the potential to reduce future disability (Keystone et al., 2004; Maini et al., 1999; Weinblatt et al., 2003; Weinblatt et al., 1999). However, these agents are expensive compared to the traditional DMARDs. Based on the average US wholesale price, adalimumab and etanercept cost around $\$ 16,000$ and infliximab costs $\$ 17,000$ per year (Drug Topics Red Book, 2004). These costs do not include pre-therapy testing for opportunistic infections or monitoring for treatment complications. Also, a number of patients (26$34 \%$ ) discontinue treatment in the first year due to toxicities or ineffectiveness (Flendrie, Creemers, Welsing, Den Broeder, \& Van Riel, 2003). A cost-effectiveness analysis of these new treatments is thus warranted. The resulting cost-effectiveness ratio will express the relationship between the extra costs and additional benefits of these new treatments in comparison with the traditional DMARDs they replace.

\section{Economic Model for RA}

In RA, the health benefits and economic costs of treatments are more evident in the longer term. However, data on the efficacy of TNF inhibitors are limited and only available from short-term RCTs. Therefore, decision analytical modeling is used to synthesize short-term clinical trial results with long-term disease epidemiological aspects, quality of life, mortality, and resource use (Bansback et al., 2005). In the last few years, a few economic studies assessing the incremental cost-effectiveness of TNF inhibitors versus standard therapies have been published. As discussed in Chapter 2, these studies have been limited by their study duration, failing to include withdrawal of patients in the first year of treatment due to lack of efficacy or toxicities, or failing to 
include additional costs due to monitoring or treating for complications in patients using TNF inhibitors. Additionally, no study has evaluated the cost-effectiveness of all three TNF inhibitors in RA patients that inadequately respond to MTX.

The current study utilized results from RCTs that were conducted in patients that inadequately responded to MTX alone. Outcome Measures in Rheumatology (OMERACT) acknowledge that the underlying characteristics of the clinical trial should define the population to which the economic analysis is applicable (Bansback et al., 2005). A hypothetical cohort of 55-year old women was selected as the base-case population who had failed two DMARDs, one of which was MTX. The study model used cost per QALY as the outcome measure.

\section{Review of Study Results}

A Markov Model was constructed to evaluate the total costs and effects of five treatments: adalimumab plus MTX, etanercept plus MTX, infliximab plus MTX, leflunomide plus MTX, and a standard therapy of MTX in patients with RA that inadequately respond to MTX alone. The model was evaluated using a first order Monte Carlo simulation. The base-case simulation model yielded an ICER of $\$ 49,724 /$ QALY for etanercept plus MTX followed by the next nondominated option of leflunomide plus MTX with an ICER of \$52,833/QALY. Infliximab plus MTX was dominated by adalimumab plus MTX and adalimumab plus MTX was ruled out through extended dominance. Thus, the net societal economic cost of etanercept plus MTX in treatment of patients that inadequately respond to MTX alone is at most 
$\$ 49,724$ to increase life expectancy by one quality-adjusted life year, i.e., one year of perfect health.

Taking only total costs into account, the standard MTX treatment was the least expensive $(\$ 82,956$ for a lifetime treatment) and etanercept plus MTX was the most expensive treatment $(\$ 314,895)$. The two other TNF inhibitors, infliximab plus MTX $(\$ 189,145)$ and adalimumab plus MTX $(\$ 187,748)$ were less expensive than etanercept plus MTX but were more expensive than leflunomide plus MTX $(\$ 116,991)$. The total QALY gain was the highest in etanercept plus MTX (15.84 QALYs) followed by adalimumab plus MTX (13.28 QALYs), leflunomide plus MTX (12.94 QALYs), infliximab plus MTX (12.51 QALYs), and standard MTX treatment (12.29 QALYs).

As this study was the first to evaluate the cost-effectiveness of all three TNF inhibitors and leflunomide in patients with RA that inadequately respond to MTX alone, results were not directly comparable to other studies. As reported earlier, there are few economic studies that have compared each of the TNF inhibitor to a standard therapy. Also, the decision analytic models used in these studies have used disparate time horizons, comparators, quantities of drugs, discount rates, and treatment sequences making it very difficult to compare the cost-effectiveness ratios between the analyses.

Reports of the cost-effectiveness of TNF inhibitors and leflunomide are available from other countries (Bansback et al., 2004; Barton et al., 2004; Brennan et al., 2003; Jobanputra et al., 2002; Kobelt et al., 2003; Kobelt et al., 2002). Different 
methodologies were employed in each of these studies to extrapolate long-term HAQ scores for patient cohorts. Kobelt and colleagues (2003) and Wong and colleagues (2002) used patient level data from infliximab clinical trial (ATTRACT) and epidemiological database to calculate Markov transition rates. Brennan and colleagues (2003) utilized clinical data and published annual progression rates to measure mean HAQ improvements for both responders and nonresponders of treatment. Jobanputra and others (2002) did not include differential disability progression in the model.

The study by Bansback and others (2004) modeled patients with moderate to severe RA who had failed at least two traditional DMARDs and estimated incremental QALY versus traditional DMARDs at 1.20 and 1.25 for adalimumab plus MTX and etanercept plus MTX, respectively. The cost-effectiveness was estimated at 40,875 euros per QALY (US \$53,137/QALY) for adalimumab plus MTX and 51,976 euros (US \$67,568/QALY) for etanercept plus MTX.

Brennan and colleagues (2003) conducted a cost-effectiveness analysis of etanercept monotherapy under British Society for Rheumatological guidelines. The study compared a DMARD sequence with etanercept as a third-line agent against the same sequence excluding etanercept. The study reported a cost-effectiveness ratio of $£ 16,330 /$ QALY (US \$26,000/QALY; costs adjusted to 2004 values) suggesting that etanercept was cost-effective when compared with non-biologic agents. The costeffectiveness ratio in the sensitivity analysis ranged from $£ 7,800$ to $£ 42,000$ (US $\$ 11,232$ to $\$ 60,480)$. 
The study by Kobelt and colleagues (2002) investigated the cost-effectiveness of leflunomide compared to MTX in the UK. The analyses covered a timeframe of 10 years. Leflunomide was reported to have costs of $£ 44,017$ (US $\$ 63,384$ ) and effects of 4.307 QALY compared to $£ 44,988$ (US \$64,782) and effects of 4.158 QALY for MTX. Another study by Kobelt and colleagues (2003) evaluated the cost per QALY of infliximab plus MTX with MTX alone in patients with advanced disease. Including both direct and indirect costs, the cost per QALY over 10 years was 3, 440 euros (US 4,953 ) in Sweden and 34,800 euros (US \$50,112) in UK. The respective QALY gains were 0.248 and 0.298 . However, these analyses included markedly different assumptions regarding treatment effectiveness, disease progression, and quality adjustments.

Studies by Jobanputra and colleagues (2002) and Barton and colleagues (2004) assessed the additional costs and QALYs gain associated with the use of either etanercept or infliximab as the third DMARD in a sequence of DMARDs. The first study resulted in an ICER of approximately $£ 83,000$ (US $\$ 130,000$; costs adjusted to 2004 values) per QALY for etanercept and approximately $£ 115,000$ (US $\$ 180,000$; costs adjusted to 2004 values) per QALY for infliximab. The second study incorporated the effects of DMARDs on joint replacement, mortality, and QoL in the model developed by Jobanputra and colleagues (2002) and reported an ICER of approximately $£ 59,289$ (US $\$ 85,376$ ) per QALY for etanercept and approximately $£ 76,233$ (US \$109,775) per QALY for infliximab was reported. Wong and colleagues (2002) examined costs and QALYs of infliximab plus MTX over a patient's lifetime 
using Markov model. The study reported an ICER of $\$ 31,000 /$ QALY (costs adjusted to 2004 values).

Despite lack of homogeneity in the methodological approaches and different model assumptions, these studies do provide a benchmark against which to compare the present study results. The discounted incremental costs per QALY estimates from these analyses for different treatments range from $\$ 26,000$ to $\$ 109,755$. Measurement of costs in the present study was comprehensive and included costs due to monitoring, costs of treating mild/moderate events, costs of treating severe adverse events, hospitalization costs and laboratory tests costs. This may explain the higher ICER of etanercept plus MTX compared with results of some of economic studies discussed earlier in the section. In terms of improvement in HRQoL, the incremental QALY reported for etanercept was 1.660 (Brennan et al., 2003), 0.214 (Jobanputra et al., 2002), and 0.248 (Kobelt et al., 2003); 0.116 for infliximab (Jobanputra et al., 2002) and 0.290 (Wong et al., 2002). The incremental QALY gain against standard MTX treatment in the present study was 3.55 for etanercept plus MTX, 0.99 for adalimumab plus MTX, 0.65 for leflunomide plus MTX, and 0.22 for infliximab plus MTX.

During the last two decades, cost-effectiveness ratios of less than or equal to $\$ 50,000$ per discounted QALY have been considered as acceptable, ratios greater than $\$ 50,000$ and less than or equal to $\$ 100,000$ per discounted QALY have been considered as reasonable, and ratios greater than $\$ 100,000$ per discounted QALY have been considered as unreasonable or high (Maetzel, 2004). Recent studies in different disease 
areas have found cost-effectiveness ratios of $\$ 60,000$ per discounted QALY for chronic hemodialysis and $\$ 1,000,000$ for interferon beta for multiple sclerosis (Wong et al., 2002). In the present study, the ICER of etanercept plus MTX, and the next nondominated treatment, leflunomide plus MTX, fall within the acceptable range of $\$ 50,000$ and $\$ 100,000$. In the sensitivity analysis, the base-case results were sensitive to variations in utilities and medication costs of etanercept and leflunomide. The lowest cost/QALY for etanercept plus MTX and leflunomide plus MTX were \$30,371 and $\$ 43,720$, respectively and the highest cost/QALY values were $\$ 74,628$ and $\$ 63,126$. These ICERs for both treatments remain below the acceptable range of $\$ 50,000$ and $\$ 100,000$.

There are conflicting reports regarding the effect of RA on the risk of mortality. The OMERACT statement recommends that the risk of mortality be included in the decision analytical modeling studies. Wong and colleagues (2002) assumed that an increase in disability level was equivalent to a 1.77 -fold increase in mortality risk. Removing this assumption, the base-case results increased the base-case cost per QALY from $\$ 30,690$ to $\$ 35,800$ (1998 values). The study by Brennan and others (2003) estimated the impact of the change in HAQ score on mortality via a COX proportional hazard regression. Kobelt and colleagues (2003) did not account for increased risk of mortality in their model and used age- and gender-adjusted all cause mortality rates. To test the effect of mortality assumption, Jobanputra and colleagues (2002) assumed standardized mortality ratios (SMR) of 1.1 and 2.1. These made no difference to the ICERs. The ICER for etanercept against standard treatment changed 
from $£ 71,659$ /QALY to $£ 71,471$ with SMR 1.1 and to $£ 71,838$ with SMR 2.1. In the present study, the incremental cost per QALY was calculated by adjusting the age-, gender-, race-adjusted all cause mortality rates by a factor of two $(\mathrm{SMR}=2)$ to reflect the increased risk of mortality due to RA. The dominance status remained the same though the incremental cost per QALY for etanercept plus MTX increased from \$49,724/QALY to \$50,782/QALY and leflunomide plus MTX decreased slightly from $\$ 52,833 / \mathrm{QALY}$ to $\$ 51,905 / \mathrm{QALY}$.

The indirect costs associated from disability associated with rheumatoid arthritis are substantial (Callahan, 1998), including unemployment with increasing disease duration, decreased productivity, and increased work absenteeism (Wong et al., 2002). The inclusion of productivity costs in economic analyses remains contentious and even OMERACT states that all direct costs and societal costs should be reported separately. Therefore, the present study reported base-case results separately for directs costs and for societal (total costs). Considering only direct costs, the dominance status remained the same for both, all cause mortality rate and RA-adjusted mortality rate. The incremental cost per QALY for etanercept plus MTX was \$24,333/QALY and \$24,421/QALY for all cause mortality rate and RA-adjusted mortality rate, respectively. Similarly, the incremental cost per QALY for leflunomide plus MTX was \$27,717/QALY and \$28,127/QALY for all cause mortality rate and RA-adjusted mortality rate, respectively. 
The study population included in this analysis was patients with severe RA that were refractory to standard therapy. Due to their disease severity, the indirect costs of these patients are expected to be higher for the patients with mild RA or newly diagnosed RA (Kavanaugh et al., 1996). Since the estimation of indirect costs is severely limited by data availability, indirect costs in the present study were considered to be one to three times that of direct costs. In the base-case analysis, indirect costs were considered equal to direct costs (Wong et al., 2002). When indirect costs were considered as twice that of direct costs, etanercept plus MTX had the lowest ICER followed closely by leflunomide plus MTX. However, when indirect costs were considered as three times that of direct costs, leflunomide plus MTX has a lowest ICER than etanercept plus MTX. In both scenarios, infliximab plus MTX and adalimumab plus MTX were ruled out by dominance and extended dominance, respectively. The ICER became favorable for leflunomide plus MTX at higher total costs since leflunomide plus MTX had lower direct costs compared to etanercept plus MTX. However, even after considering high indirect costs, the ICERs of etanercept plus MTX and leflunomide plus MTX seemed to be in a reasonable range of $\$ 75,000$ - $\$ 100,000$.

One of the main contributors to the high cost-effectiveness ratios may be the high acquisition cost of the TNF inhibitors, which if lowered, would make the costeffectiveness ratios even more acceptable. However, no rheumatologist, knowing the important role and value of TNF inhibitors would want cost-effectiveness ratios to influence the way patients receive these treatments. A study by Erkan and colleagues (2002) demonstrated that economic factors did influence DMARD preferences. 
However, in addition to the cost of treatment, a number of other factors have been found to influence a rheumatologist's decision-making process regarding choice of therapy. These include patient preference, the disease itself (for example, disease duration and symptom severity), the drug chosen (for example, potential adverse events, cost, and route of administration), and published evidence documenting the overall experience with each drug (Schwartzman et al., 2004; Schwartzman \& Morgan, 2004). No study has assessed rheumatologist's prescribing patterns and the factors that influence rheumatologist's choice of a particular TNF inhibitor over less costly standard RA treatments. The lack of information on the actual use of TNF inhibitors by rheumatologists led to the development of phase II of this study. 


\section{Phase II}

Phase II of the study involved a cross-sectional mail survey of randomly selected national sample of rheumatologists. The study assessed the current rheumatologists' prescribing patterns, their laboratory monitoring guidelines, and the potential barriers they face in terms of using TNF inhibitors or making a choice among the three agents. The mailing list had a total of 1,970 rheumatologists. Trainees, nonclinical, and those in pediatric practices, were excluded from the survey. A survey instrument developed for this study was mailed to each rheumatologist. Table 31 gives the response rate analysis. Of the 1,970 surveys mailed, 48 were undeliverable due to incomplete or incorrect addresses, death or retirement of the rheumatologist. Thus, 1,922 rheumatologists were presumably reached by the mailings. A total of 432 usable responses were returned after two mailings for a response rate of $22.5 \%$. Four surveys were excluded from the analysis because they had less than $50 \%$ complete item responses, thereby reducing the response rate to $22.3 \%$. 
Table 31. Response rate

\begin{tabular}{llrc}
\hline & & $\mathrm{N}$ & Percent (\%) \\
\hline Original sample size & & 1,970 & \\
Undeliverable surveys & Effective Sample Size & $\mathbf{1 , 9 2 2}$ & 100.0 \\
\multicolumn{2}{l}{$\begin{array}{l}\text { Number of questionnaires returned } \\
\text { Incomplete questionnaire }\end{array}$} & 432 & 22.5 \\
& Usable Response & 4 & \\
& Usable Response Rate & & \\
& & & \\
\end{tabular}




\section{Non-response Analysis}

The nonresponse survey was mailed to a randomly selected sample of rheumatologists who did not respond to the two mailings of the survey. Of the 200 nonrespondent surveys mailed, 40 were returned, thus giving a response rate of $20 \%$. The reasons cited by the rheumatologists for their nonparticipation in the study are listed in Table 32. The most common reasons cited were: do not respond to mail surveys $(35 \%)$, did not have enough time to complete the survey $(30 \%)$, the survey was too long $(15.4 \%)$, forgot the survey $(10 \%)$, no incentive to participate $(7.5 \%)$, did not receive the survey $(5 \%)$, not interested in such studies $(5 \%)$, the survey was misplaced (7.5\%), and other reasons $(22.5 \%)$.

Demographics and practice-related characteristics of nonrespondents are presented in Table 33. Of the 40 rheumatologists who responded to the non-response survey, $61 \%$ were males and $39 \%$ were females. The mean age was $46.8(\mathrm{SD}= \pm 8.5)$ years. The mean number of years in practice as a rheumatologist was $14.6(\mathrm{SD}= \pm 8.7)$ years and the mean number of patients with RA seen per week was $26.3(\mathrm{SD}= \pm 13.1)$. A total of $66.7 \%$ described their primary practice site as group-based, $19.4 \%$ as solobased, $8.3 \%$ as university-affiliated hospital, $2.8 \%$ as hospital-based, and $2.8 \%$ as other. Rheumatologists reported using all three agents in patients with varying disease severity. Rheumatologists prescribed all three agents in patients with moderate RA (88-100\%) and severe RA (88-97\%). Also, some rheumatologists (11-26\%) reported using these agents in newly diagnosed and mild RA patients (Table 34). 
Table 32. Reasons for not participating in the study survey

\begin{tabular}{lll}
\hline Reasons for Non Participation & $\mathrm{N}(40)^{*}$ & Percent (\%) \\
\hline Do not respond to mail surveys & 14 & 35.0 \\
Did not have enough time to complete the survey & 12 & 30.0 \\
The survey was too long & 6 & 15.4 \\
Forgot the survey & 4 & 10.0 \\
No incentive to participate & 3 & 7.5 \\
The survey was misplaced & 3 & 7.5 \\
Did not receive the survey & 2 & 5.0 \\
Not interested in such studies & 2 & 5.0 \\
Other & 9 & 22.5 \\
\hline
\end{tabular}

* Multiple responses were checked by responders of nonresponse survey, hence total greater than $100 \%$ 
Table 33. Demographic and practice-related characteristics of respondents of the non-response survey

\begin{tabular}{lll}
\hline Characteristics & $\mathrm{N}$ & Percent (\%) \\
\hline Age, years (Mean $\_$S.D) & $46.8 \pm 8.5$ & \\
Gender & 22 & 61.1 \\
$\quad$ Males & 14 & 38.9 \\
$\quad$ Females & 1 & 2.8 \\
Primary practice site & 3 & 8.3 \\
$\quad$ Hospital-based & 7 & 19.4 \\
$\quad$ University affiliated hospital & 24 & 66.7 \\
$\quad$ Solo-based & 1 & 2.8 \\
$\quad$ Group-based & & \\
$\quad$ Other & $14.6 \pm 8.7$ & \\
$\begin{array}{l}\text { Number of years in practice as a } \\
\text { rheumatologist (Mean } \pm \text { S.D) }\end{array}$ & $26.3 \pm 13.1$ & \\
$\begin{array}{l}\text { Number of patients with RA seen } \\
\text { per week (Mean } \pm \text { S.D) }\end{array}$ &
\end{tabular}

S.D $=$ Standard deviation, $\mathrm{RA}=$ Rheumatoid arthritis 
Table 34. Patient population in which TNF inhibitors used by respondents of the non-response survey*

\begin{tabular}{lcccc}
\hline $\begin{array}{l}\text { TNF } \\
\text { inhibitors }\end{array}$ & $\begin{array}{c}\text { Newly Diagnosed } \\
\mathrm{N}(\%)\end{array}$ & $\begin{array}{c}\text { Mild RA } \\
\mathrm{N}(\%)\end{array}$ & $\begin{array}{c}\text { Moderate RA } \\
\mathrm{N}(\%)\end{array}$ & $\begin{array}{c}\text { Severe RA } \\
\mathrm{N}(\%)\end{array}$ \\
\hline Adalimumab & $6(17.6)$ & $7(20.6)$ & $33(97.1)$ & $32(94.1)$ \\
Etanercept & $9(26.5)$ & $8(23.5)$ & $34(100)$ & $33(97.1)$ \\
Infliximab & $4(11.8)$ & $6(17.6)$ & $30(88.2)$ & $30(88.2)$ \\
\hline
\end{tabular}

${ }^{*}$ Multiple responses were checked by responders of nonresponse survey, hence total greater than $100 \%$ $\mathrm{TNF}=$ Tumor necrosis factor, $\mathrm{RA}=$ Rheumatoid arthritis 
Respondents and nonrespondents were compared on the basis of their demographic and practice-related characteristics. The variables compared were age, gender, primary practice site, number of years in practice as a rheumatologist, and mean number of patients with RA seen per week. Independent $t$-tests and Chi-square tests were conducted for comparisons of these variables. The result of the analysis is presented in Table 35. The respondents and nonrespondents were significantly different on age and years of practice. The nonrespondents were younger and had less number of years in practice as a rheumatologist compared to the respondents.

However, no significant differences were noted between respondents and nonrespondents for gender, primary practice site, and average number of patients with RA seen each week. 
Table 35. Analysis of non-response bias for demographic and practice-related characteristics

\begin{tabular}{|c|c|c|c|c|}
\hline Characteristics & Respondents & $\begin{array}{l}\text { Non- } \\
\text { respondents }\end{array}$ & $\begin{array}{l}\text { Test } \\
\text { Statistic }\end{array}$ & $\begin{array}{l}\text { Significance } \\
\mathrm{P}\end{array}$ \\
\hline Age, years (Mean_S.D) & $50.9 \pm 9.4$ & $46.8 \pm 8.5$ & $\mathrm{t}=2.492$ & $0.013^{*}$ \\
\hline Gender, n (\%) & & & $\chi^{2}=3.293$ & 0.07 \\
\hline Males & $323(93.6)$ & $22(6.4)$ & & \\
\hline Females & $108(88.5)$ & $14(11.5)$ & & \\
\hline Primary practice site, $\mathrm{n}(\%)$ & & & $\chi^{2}=5.389$ & 0.25 \\
\hline Hospital-based & $18(94.7)$ & $1(5.3)$ & & \\
\hline $\begin{array}{l}\text { University-affiliated } \\
\text { hospital }\end{array}$ & $75(96.2)$ & $3(3.8)$ & & \\
\hline Solo-based & $111(94.1)$ & $7(5.9)$ & & \\
\hline Group-based & $223(90.3)$ & $24(1.8)$ & & \\
\hline Other & $3(75)$ & $1(25)$ & & \\
\hline $\begin{array}{l}\text { Number of years in practice a } \\
\text { rheumatologist (Mean+S.D) }\end{array}$ & $18.2 \pm 9.6$ & $14.6 \pm 8.7$ & $\mathrm{t}=2.211$ & $0.028^{*}$ \\
\hline $\begin{array}{l}\text { Number of patients with RA } \\
\text { seen per week (Mean } \_ \text {S.D) }\end{array}$ & $29.9 \pm 20.1$ & $26.3 \pm 13.1$ & $\mathrm{t}=1.009$ & 0.314 \\
\hline
\end{tabular}

* Significant at $\mathrm{p}<0.05, \mathrm{~S} . \mathrm{D}=$ Standard deviation, $\mathrm{RA}=$ Rheumatoid arthritis 


\section{Demographics and Practice-related Characteristics of Responding Rheumatologists}

Demographics and practice-related characteristics of respondents are presented in Table 36. Of the 432 respondents, $25.1 \%$ were women and $74.9 \%$ were men. Mean age in years $(\mathrm{S} . \mathrm{D})$ was $50.9(\mathrm{SD}= \pm 9.4)$, mean number of years in practice as a rheumatologist was $18.2(\mathrm{SD}= \pm 9.6)$, and mean number of patients with $\mathrm{RA}$ seen per week was $29.9(\mathrm{SD}= \pm 20.1)$. Eighteen $(4.2 \%)$ rheumatologists reported their practice site as hospital based, 75 (17.4\%) as university-affiliated hospital, $111(25.8 \%)$ as solo office-based, $223(51.9 \%)$ as group office-based, and three $(0.7 \%)$ as other practice sites. 
Table 36. Demographic and practice-related characteristics of respondents

\begin{tabular}{lll}
\hline Characteristics & N & Percent (\%) \\
\hline
\end{tabular}

Age, years $($ Mean \pm S.D)

$50.9 \pm 9.4$

Gender

Males

323

Females

108

Primary practice site

$\begin{array}{lrr}\text { Hospital-based } & 18 & 4.2 \\ \text { University affiliated hospital } & 75 & 17.4 \\ \text { Solo-based } & 111 & 25.8 \\ \text { Group-based } & 223 & 51.9 \\ \text { Other } & 3 & 0.7\end{array}$

Number of years in practice as a

rheumatologist (Mean+S.D)

$18.2+9.6$

Number of patients with RA

seen per week (Mean+S.D)

$29.9 \pm 20.1$

S.D = Standard deviation, $\mathrm{RA}=$ Rheumatoid arthritis 


\section{Results for Objective 1}

The questionnaire asked the respondents to indicate their use of specific TNF inhibitors in different groups of patients with RA. For study purposes, four groups of patients were identified based on the severity of the disease: newly diagnosed, mild RA, moderate RA, and severe RA. Rheumatologists reported using all three TNF inhibitors in patients with moderate RA (82-90\%) and severe RA (94-96\%). Also, some rheumatologists (10-18\%) reported using these agents in newly diagnosed and patients with mild RA (Table 37).

The questionnaire also asked rheumatologists regarding their use of TNF inhibitors in patients with severe RA that responded inadequately to methotrexate alone. The response was based on the use of TNF inhibitors reported in clinical practice: TNF inhibitor alone, used in combination with one disease modifying antirheumatic drug (DMARD), used in combination with two other DMARDs, TNF inhibitors not used, and other treatments or approaches used. Rheumatologists were asked to check either one or more responses based on their use of TNF inhibitors in this patient population. A majority of rheumatologists $(n=392 ; 91.2 \%)$ reported using a TNF inhibitor with one other (DMARD), 120 (28\%) used a TNF inhibitor with two other DMARDs, $73(17.0 \%)$ rheumatologists reported using a TNF inhibitor as monotherapy, $11(2.6 \%)$ reported not using any TNF inhibitors in such patients, and 35 $(8.2 \%)$ used other treatments or approaches. 
Table 37. Respondents prescribing TNF inhibitors in patients based on the severity of the disease*

\begin{tabular}{lcccc}
\hline $\begin{array}{l}\text { TNF } \\
\text { inhibitors }\end{array}$ & $\begin{array}{c}\text { Newly Diagnosed } \\
\mathrm{N}(\%)\end{array}$ & $\begin{array}{c}\text { Mild RA } \\
\mathrm{N}(\%)\end{array}$ & $\begin{array}{c}\text { Moderate RA } \\
\mathrm{N}(\%)\end{array}$ & $\begin{array}{c}\text { Severe RA } \\
\mathrm{N}(\%)\end{array}$ \\
\hline Adalimumab & $64(14.8)$ & $54(12.5)$ & $375(87.2)$ & $411(95.4)$ \\
Etanercept & $77(17.9)$ & $68(15.8)$ & $390(90.5)$ & $416(96.7)$ \\
Infliximab & $46(10.7)$ & $43(10.0)$ & $357(82.8)$ & $408(94.7)$ \\
\hline
\end{tabular}

${ }^{*}$ Multiple responses were checked by responders of nonresponse survey, hence total greater than $100 \%$ $\mathrm{TNF}=$ Tumor necrosis factor, $\mathrm{RA}=$ Rheumatoid arthritis 
Rheumatologists were asked if they had ever switched any patient from one TNF inhibitor to a different TNF inhibitor. Also, if they had switched patients to a different TNF inhibitor, their reasons for switching were elicited using an open-ended question. Over 94\% rheumatologists stated switching patients from one TNF inhibitor to a different TNF inhibitor in case of a nonresponse. The following reasons were given by the respondents for switching patients from one TNF inhibitors to another: lack of efficacy (44.9\%), adverse events (20.1\%), insurance and reimbursement issues (3.7\%), differences in the mechanism of action of the three TNF inhibitors (3.5\%), success of switching based on study reports and case studies (3.5\%), allergic reaction and infusion-site reaction to infliximab (2.1\%), cost (1.9\%), and patient preferences $(1.6 \%)$

Rheumatologists were also asked to indicate the average number of prescriptions written per week for each drug. The average number of prescriptions per week reported for adalimumab, etanercept, and infliximab were $3.68(\mathrm{SD}= \pm 5.11)$, $6.05(\mathrm{SD}= \pm 8.99)$, and $4.22(\mathrm{SD}= \pm 8.24)$, respectively. 


\section{Discussion for Objective 1}

Modern RA management stresses the need for an aggressive treatment

preferably at an early stage of the disease. The original labeling of the TNF inhibitors indicates their use in patients with moderate to severely active RA despite treatment with MTX. However, there are some emerging clinical reports that indicate their success in patients who have been recently diagnosed or those who have a mild form of the disease (Genovese, Bathon, \& Martin, 2002; Smolen, ven der Heijde, Emery et al., 2004). While there is a tremendous excitement regarding the potential for disease modification in those patients with an early forms of the disease, there are issues such as costs and access that restrict the use of these agents in patients with early form of disease. Results of this study show that a majority of rheumatologists are prescribing TNF inhibitors for moderate to severe patients as indicated. However, there appears to be some rheumatologists who seem to broaden the official indication of TNF inhibitors to patients with early-onset RA.

For the most severe group of patients with RA that inadequately respond to MTX alone, it is not known if the TNF inhibitors are being prescribed alone or used in combination with other DMARDs, or are being used as a second- or a third-line therapy. Recent results from clinical trials have demonstrated a synergistic effect of MTX and TNF inhibitors in reducing signs and symptoms of RA, inhibiting structural damage, and improving physical functions in patients with RA (Klareskog et al., 2004; Smolen et al., 2004). Thus, a combination of TNF inhibitors with MTX has become somewhat of a standard for patients with aggressive disease (Choy, 2004; Schnabel, 
2004). The result of this study is consistent with the reports that TNF inhibitor with one DMARD is the most commonly used combination followed by a combination of TNF with two DMARDs. Some rheumatologists reported using TNF inhibitors alone while others reported using other treatments or approaches in treating patients with severe disease.

Not all patients respond to TNF inhibitors and some who do respond develop treatment limiting side-effects. Since the three TNF inhibitors seem to have similar efficacies in RA, an important practical question for clinicians is whether there is a rationale for prescribing another TNF inhibitor in case of a failure with the first TNF inhibitor or whether this practice only increases treatment costs (van Vollenhoven, 2004). The results indicate that an overwhelming majority of rheumatologists switched patients from one TNF inhibitor to another. Major reasons for switching included lack of efficacy or adverse events associated with the TNF inhibitor. Kavanaugh and colleagues (2004) have reported that reasons for switching patients from one TNF inhibitor to another are varied and include toxicity, loss of efficacy, cost, and reimbursement issues.

Other reasons for switching could include the differences in mechanism of action of the TNF inhibitors. Tumor necrosis factor (TNF- $\alpha$ ), the proinflammatory cytokine, is believed to be an important factor in determining clinical response. Etanercept is made of two P75 soluble receptors and is capable of binding to both TNF$\alpha$ and TNF- $\beta$ while infliximab and adalimumab are monoclonal antibodies and specific 
to TNF- $\alpha$. Infliximab is administered intermittently in large doses and after achieving peak levels, decrease to undetectable levels after 6 to 8 weeks. This warrants shortening of intervals of infusions in patients who do not respond to the 8-week interval. On the other hand, shorter half life of etanercept and its administration twice a week leads to more sustained levels that may be inadequate in patients who require more rapid and drastic suppression. This subset of patient population may benefit most from a switch to infliximab (Haraoui, 2004). However, much remains to be learned about the exact mechanisms of these agents and until predictors of response have been identified, the use of these agents and switching between them will be governed by clinical judgment and accumulated experience. 


\section{Results for Objective 2}

Rheumatologists ranked, in order of preference, the TNF inhibitors they would give to patients in whom one is determined as necessary. Etanercept was ranked first by $292(68.2 \%)$ respondents, adalimumab by 120 (28.2\%), and infliximab by 86 (20.4\%) respondents (Table 38). Of those who ranked etanercept as first choice $(n=292), 38(13 \%)$ and $33(11.5 \%)$ also ranked adalimumab and infliximab as first choice, $159(54.5 \%)$ and $97(33.2 \%)$ ranked adalimumab and infliximab as second choice, and $90(31.0 \%)$ and $154(53.5 \%)$ ranked adalimumab and infliximab as third choice.

Of those who ranked adalimumab as first choice $(n=120), 38(31.7 \%)$ and 32 (26.7\%) also ranked etanercept and infliximab as first choice, $71(59.2 \%)$ and 15 $(12.5 \%)$ ranked etanercept and infliximab as second choice, and $11(9.2 \%)$ and 69 (58.5\%) ranked etanercept and infliximab as third choice. Of those who ranked infliximab as first choice $(n=86), 32(37.2 \%)$ and $33(38.4 \%)$ also ranked adalimumab and etanercept and as first choice, $22(25.6 \%)$ and $32(37.2 \%)$ ranked adalimumab and etanercept as second choice, and $32(37.2 \%)$ and $21(24.4 \%)$ ranked adalimumab and etanercept as third choice. 
Table 38. Ranking of TNF inhibitors based on rheumatologist's preference

\begin{tabular}{llll}
\hline TNF inhibitors & $\begin{array}{l}\text { Rank 1 } \\
\mathrm{N}(\%)\end{array}$ & $\begin{array}{l}\text { Rank 2 } \\
\mathrm{N}(\%)\end{array}$ & $\begin{array}{l}\text { Rank 3 } \\
\mathrm{N}(\%)\end{array}$ \\
\hline Adalimumab & $120(28.2)$ & $181(42.5)$ & $122(28.6)$ \\
Etanercept & $292(68.2)$ & $104(24.3)$ & $32(7.5)$ \\
Infliximab & $86(20.4)$ & $107(25.4)$ & $223(53.0)$ \\
\hline
\end{tabular}

Rank 1: First choice agent, $\mathrm{TNF}=$ Tumor necrosis factor 


\section{Discussion for Objective 2}

Etanercept was ranked first by $292(68.2 \%)$ respondents, adalimumab by 120 (28.2\%) and infliximab by $86(20.4 \%)$. Around $42.5 \%$ and $53 \%$ of the respondents ranked adalimumab and infliximab as their second and third choice TNF inhibitor, respectively. Thus, this indicates a clear preference for etanercept as the first choice TNF inhibitor compared to the other two TNF inhibitors among the responding rheumatologists. Thus, the null hypothesis A that rheumatologists do not have any preference for any of the TNF inhibitors is rejected.

The analysis revealed that there is a preference for etanercept and that infliximab is the third choice agent among the three TNF inhibitors for a majority of the rheumatologists. The preference for etanercept could be due to the fact that it was the first TNF inhibitor to be launched in the market in the year 1999 and therefore, has the most clinical use experience among the three agents. Also, data from clinical trials, practice experiences, and post-marketing surveillance reports have shown that etanercept is effective and quite safe (Fleischmann \& Yocum, 2004). 


\section{Results for Objective 3}

Rheumatologists who preferred adalimumab, etanercept, or infliximab as their first choice agent were compared on the basis of their demographic (age, gender), practice-related characteristics (primary practice site, number of years in practice as a rheumatologist, and average number of patients with RA seen per week), and perceived barriers to prescribing the TNF inhibitors. Analysis of variance (ANOVA) and Chisquare tests were conducted to compare these variables. Significant ANOVAs were further investigated by post-hoc Tukey comparisons. Since the analysis compared rheumatologists who preferred adalimumab, etanercept, or infliximab as their first choice agent, rheumatologists who ranked all the three TNF inhibitors as their first choice agent were excluded from the analysis $(n=32)$.

Table 39 gives the results of the analysis. Rheumatologists who preferred adalimumab, etanercept, or infliximab as their first choice agent were significantly different on gender and number of patients with RA seen per week. Rheumatologists who preferred infliximab as their first choice agent saw more patients with RA per week compared with rheumatologists who preferred adalimumab or etanercept as the first choice TNF inhibitor. Also, rheumatologists who preferred infliximab perceived support for administration of infusion as a major problem compared with those who either preferred adalimumab or etanercept. Rheumatologists who preferred etanercept perceived patient compliance with the agent as a major problem compared with those who preferred adalimumab. 
Table 39. Demographic, practice-related characteristics, and perceived barriers of rheumatologists who preferred adalimumab, etanercept, or infliximab as their first choice TNF inhibitor

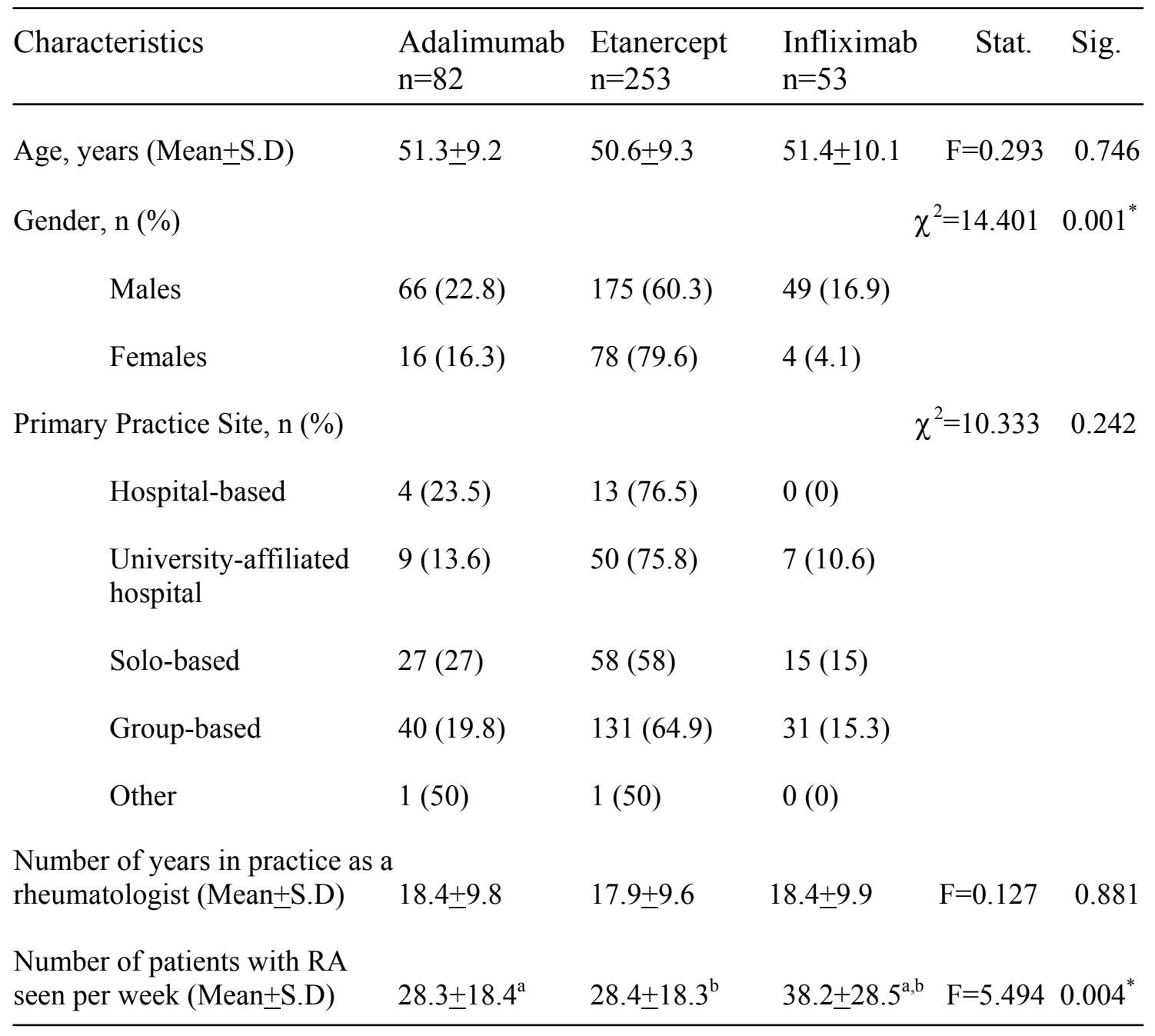

ANOVA: Significant ANOVA's were investigated with post-hoc Tukey comparisons $a, b$ : Means with same letters are significantly different.

* Significant at $\mathrm{p} \leq 0.05, \mathrm{~S} . \mathrm{D}=$ Standard deviation, $\mathrm{RA}=$ Rheumatoid arthritis 
Table 39 (contd.). Demographic, practice-related characteristics, and perceived barriers of rheumatologists who preferred adalimumab, etanercept, or infliximab as their first choice TNF inhibitor

\begin{tabular}{lllllc}
\hline & $\begin{array}{l}\text { Adalimumab } \\
\text { Factors }\end{array}$ & Etanercept & Infliximab & Stat. & Sig. \\
\hline Age of Patient & $($ Mean \pm S.D $)$ & $($ Mean \pm S.D $)$ & $($ Mean \pm S.D $)$ & & \\
Costs to Patients & $2.3 \pm 1.6$ & $2.3 \pm 1.6$ & $2.1 \pm 1.5$ & $\mathrm{~F}=0.538$ & 0.584 \\
Efficacy of TNF inhibitors & $2.1 \pm 1.3$ & $2.4 \pm 1.7$ & $2.1 \pm 1.3$ & $\mathrm{~F}=2.091$ & 0.125 \\
Insurance/Formulary coverage & $5.0 \pm 1.7$ & $4.9 \pm 1.9$ & $4.9 \pm 1.9$ & $\mathrm{~F}=0.356$ & 0.701 \\
Patient Compliance & $1.8 \pm 1.0^{\mathrm{a}}$ & $2.2 \pm 1.4^{\mathrm{a}}$ & $1.9 \pm 1.2$ & $\mathrm{~F}=5.140$ & $0.006^{*}$ \\
Patient Preference & $2.1 \pm 1.5$ & $2.5 \pm 1.4$ & $2.5 \pm 1.5$ & $\mathrm{~F}=2.615$ & 0.074 \\
Route of Administration & $2.4 \pm 1.4$ & $2.7 \pm 1.5$ & $2.8 \pm 1.5$ & $\mathrm{~F}=2.082$ & 0.126 \\
Side Effects & $2.8+1.2$ & $2.9 \pm 1.4$ & $3.2 \pm 1.4$ & $\mathrm{~F}=2.524$ & 0.081 \\
Support for Administration & $1.5 \pm 0.8^{\mathrm{b}}$ & $1.7 \pm 1.3^{\mathrm{c}}$ & $2.2 \pm 1.5^{\mathrm{b}, \mathrm{c}}$ & $\mathrm{F}=6.861$ & $0.001^{*}$ \\
\hline
\end{tabular}

Measured on a 7-point scale where $1=$ no problem and $7=$ major problem

ANOVA: Significant ANOVA's were investigated with post-hoc Tukey comparisons

a,b,c: Means with same letters are significantly different.

Significant at $\mathrm{p}<0.05$ 


\section{Discussion for Objective 3}

Based on the analysis, the null hypothesis B that there is no difference in demographic, practice-related characteristics, and perceived barriers to prescribing TNF inhibitors among rheumatologists who preferred adalimumab, etanercept, or infliximab as their first choice agent is rejected. Gender and number of patients with RA seen per week do have an association with prescribing preferences of rheumatologists. More males compared to female rheumatologists showed preference for these agents. Rheumatologists who preferred infliximab as their first choice agent saw more patients with RA per week compared with rheumatologists who preferred adalimumab or etanercept as the first choice TNF inhibitor. This could be attributed to the difference in dosing schedule and route of administration of the three drugs (Schwartzman \& Morgan, 2004). Infliximab is administered intravenously (IV) at a dose of $3 \mathrm{mg} / \mathrm{kg}$ at weeks 0,2 , and 6 , then every 8 weeks thereafter. Both adalimumab and etanercept can be self-administered subcutaneously (SC). Thus, patients on infliximab require frequent visits to the rheumatologists to get the infusion.

One of the perceived barriers for prescribing infliximab, although a minor problem, was the support needed for administering infliximab. Infliximab is generally administered on an outpatient basis and this requires the use of professionally staffed clinics and hospital infusion suite. In addition to the basic equipment necessary for administering the infusion, the possibility of an acute infusion reaction to infliximab also necessitates the availability of emergency personnel and equipment (Gallup, 2001). 
The compliance with etanercept although perceived as a minor barrier was significantly different than the other two TNF inhibitor. This perception could be attributed to a number of factors. It is seen that treatment with assisted IV infusions has higher compliance rates than self-administered SC injections. Results of a survey of Canadian patients with RA indicate a preference for IV administration (infliximab) over an SC regimen (etanercept) (Schwartzman \& Morgan, 2004). Patients receiving etanercept must receive a short training session in self-administered SC injection while, as mentioned earlier, infliximab is administered on an outpatient basis by trained staff (Gallup, 2001).

Another major reason for compliance being cited as a perceived barrier could be due to inequalities in coverage and reimbursement policies. Infliximab is given intravenously and is covered under medical benefits, which are often unlimited or have a very high cap (e.g., \$1,000,000). Self-injectables like etanercept are covered by pharmacy benefits that are limited by the caps (typically $\$ 400-\$ 500$ per year for Medicare patients). In addition, many managed care organizations institute three-tier plans that require high copay (Gallup, 2001). These reasons could potentially explain the lower compliance with etanercept perceived by rheumatologists as compared to infliximab. 


\section{Results for Objective 4}

\section{$\underline{\text { Adalimumab }}$}

The most prescribed dose for adalimumab was $40 \mathrm{mg}$ every other week. Nearly one-fourth of the rheumatologists used this dose in patients with newly diagnosed and mild RA while a majority of rheumatologists indicated using this dose in moderate RA $(83.7 \%)$ and severe RA (74.5\%). For patients with severe RA, one-fifth of the rheumatologists indicated using $40 \mathrm{mg}$ of adalimumab every week.

The laboratory tests routinely ordered by the rheumatologists varied with respect to the tests ordered and the frequency at which these were ordered. A majority of the rheumatologists ordered complete blood count (88.2\%) and purified protein test $(96.6 \%)$. Purified protein test (PPD) was ordered mostly at baseline $(82.7 \%)$ and a few $(15.7 \%)$ ordered the test after a year of follow-up. Antinuclear antibody test (ANA), chest radiography, and creatinine were monitored by 30 to $60 \%$ of rheumatologists (Table 40). 
Table 40. Laboratory tests ordered for adalimumab and percentage of rheumatologists ordering the tests during different weeks

\begin{tabular}{lllllllll}
\hline Tests & N $(\%)$ & \multicolumn{7}{c}{ Weeks $(\%)$} \\
& & 0 & 4 & 6 & 8 & 12 & 24 & 52 \\
\hline ANA & $132(32.5)$ & 52.3 & - & 1.6 & 2.3 & 3.9 & 6.3 & 20.3 \\
CBC & $358(88.2)$ & 2.5 & 9.9 & 7.6 & 22.6 & 37.3 & 5.7 & 2.5 \\
CXR & $205(50.5)$ & 71.0 & - & - & - & - & - & 24.0 \\
Creatinine & $247(61.6)$ & 7.7 & 6.9 & - & 22.7 & 32.8 & 7.7 & - \\
PPD & $392(96.6)$ & 82.7 & - & - & - & - & - & 15.7 \\
\hline
\end{tabular}

ANA - Anti-nuclear antibody, CBC - Complete blood count, CXR - Chest radiograph, PPD - Purified protein derivative 


\section{Etanercept}

The most prescribed dose for etanercept was $50 \mathrm{mg}$ every week for newly diagnosed (15.3\%) and mild RA (14.9\%) patients followed by $25 \mathrm{mg}$ twice weekly in newly diagnosed (13.7\%) and mild RA (13.2\%) patients. Nearly half of the rheumatologists reported using etanercept $25 \mathrm{mg}$ twice a week in moderate to severe patients while $40 \%$ of rheumatologists used a dose of $50 \mathrm{mg}$ every week in patients with moderate to severe RA.

Similar to tests for adalimumab, the laboratory tests routinely ordered for etanercept varied with respect to the tests ordered and the frequency at which these were ordered. A majority of the rheumatologists ordered complete blood count (88.2\%) and PPD (96.4\%). Purified protein test was ordered mostly at baseline $(82.6 \%)$ and a few $(15.9 \%)$ ordered the test after a year of follow-up. Antinuclear antibody test (ANA), chest radiography, and creatinine were monitored by 30 to $60 \%$ of rheumatologists (Table 41). 
Table 41. Laboratory tests ordered for etanercept and percentage of rheumatologists ordering the tests during different weeks

\begin{tabular}{lllllllll}
\hline Tests & N $(\%)$ & \multicolumn{7}{c}{ Weeks $(\%)$} \\
& & 0 & 4 & 6 & 8 & 12 & 24 & 52 \\
\hline ANA & $139(33.6)$ & 53.3 & - & 1.6 & 2.3 & 3.9 & 9.6 & 20.7 \\
CBC & $365(88.2)$ & - & 10.7 & 7.4 & 22.8 & - & 11.5 & - \\
CXR & $209(50.5)$ & 70.2 & - & - & - & - & - & 23.9 \\
Creatinine & $255(61.6)$ & - & 7.5 & 8.3 & 22.5 & 32.0 & 13.4 & - \\
PPD & $398(96.4)$ & 82.6 & - & - & - & - & - & 15.9 \\
\hline
\end{tabular}

ANA - Anti-nuclear antibody, CBC - Complete blood count, CXR - Chest radiograph, PPD - Purified protein derivative 


\section{$\underline{\text { Infliximab }}$}

The preferred dose for infliximab was $3 \mathrm{mg} / \mathrm{kg}$ in patient population with varying disease severity. Around $17 \%$ rheumatologists prescribed $3 \mathrm{mg} / \mathrm{kg}$ in newly diagnosed and mild RA patients while $43.4 \%$ and $34.2 \%$ prescribed $3 \mathrm{mg} / \mathrm{kg}$ in moderate and severe RA, respectively.

Rheumatologists reported using a range of doses for infliximab with almost $93 \%$ indicating the use of a minimum dose of $3 \mathrm{mg} / \mathrm{kg}$ and $67 \%$ reporting a maximum dose of $5 \mathrm{mg} / \mathrm{kg}$ for newly diagnosed patients. In mild patients, $88 \%$ rheumatologists reported using a minimum dose of $3 \mathrm{mg} / \mathrm{kg}$ and $73 \%$ reported a maximum dose of 5 $\mathrm{mg} / \mathrm{kg}$. The 8 -week dosing interval for infliximab infusion was universally used by rheumatologists in newly diagnosed $(89.3 \%)$ and mild patients $(88.5 \%)$. In moderate patients, $81 \%$ of rheumatologists used a minimum dose of $3 \mathrm{mg} / \mathrm{kg}$ while a maximum dose of $5 \mathrm{mg} / \mathrm{kg}$ was used by $52 \%$ rheumatologists and a maximum dose of $10 \mathrm{mg} / \mathrm{kg}$ was used by $19.5 \%$ of rheumatologists. Almost three-fourth of the rheumatologists reported a dosing interval of 8 weeks while $13.2 \%$ rheumatologists reported a dosing interval of 6-8 weeks between infusions.

For severe patients, rheumatologists (64\%) used a minimum dose of $3 \mathrm{mg} / \mathrm{kg}$ and $22 \%$ rheumatologists used a minimum dose of $5 \mathrm{mg} / \mathrm{kg}$ while $30 \%$ and $37 \%$ reported using a maximum dose of $5 \mathrm{mg} / \mathrm{kg}$ and $10 \mathrm{mg} / \mathrm{kg}$, respectively. In severe patients, rheumatologists reported using a dosing interval of 8 -week (55.1\%), 6-8 
weeks (15.6\%), 6-week (11.4\%), and 4-8 weeks (9.9\%) indicating an increase in the frequency of treatment.

The results for laboratory tests ordered and frequency of the tests for infliximab were similar to that of adalimumab and etanercept. Rheumatologists ordered complete blood count (89.3\%) and PPD (97\%). Purified protein test was ordered mostly at baseline (82.3\%) and a few (15.6) ordered the test after a year of follow-up. Like the PPD test, chest radiograph was ordered by $51.8 \%$ rheumatologists with $71.3 \%$ ordering them at baseline and $21.3 \%$ after a year of follow-up. Antinuclear antibody test (ANA) was ordered by $38 \%$ of rheumatologists with nearly half of them ordering the test at baseline (Table 42). 
Table 42. Laboratory tests ordered for infliximab and percentage of rheumatologists ordering the tests during different weeks

\begin{tabular}{lllllllll}
\hline Tests & N $(\%)$ & \multicolumn{7}{c}{ Weeks (\%) } \\
& & 0 & 4 & 6 & 8 & 12 & 24 & 52 \\
\hline & & & & & & & & \\
& & & & & & \\
ANA & $152(38)$ & 52.7 & - & - & 5.4 & 6.8 & 14.2 & 16.2 \\
CBC & $357(89.3)$ & - & 10.2 & 9.0 & 31.9 & 29.1 & 9.6 & - \\
CXR & $207(51.8)$ & 71.3 & - & - & - & - & - & 21.3 \\
Creatinine & $252(63)$ & 6.8 & 7.2 & 11.2 & 29.2 & 26.0 & 12.0 & - \\
PPD & $387(97)$ & 82.3 & - & - & - & - & - & 15.6 \\
\hline
\end{tabular}

ANA - Anti-nuclear antibody, CBC - Complete blood count, CXR - Chest radiograph, PPD - Purified protein derivative 


\section{Discussion for Objective 4}

The survey revealed some interesting points regarding the dosing of the TNF inhibitors and laboratory tests ordered by the rheumatologists. Dosing guidelines suggest that adalimumab patients are prescribed a dose of $40 \mathrm{mg}$ every other week, etanercept patients two doses of $25 \mathrm{mg}$ a week or a new dosage of $50 \mathrm{mg}$ once every week. The recommended dosing for infliximab is $3 \mathrm{mg} / \mathrm{kg}$ of body weight for the first dose and then at two and six weeks and every eight weeks thereafter. Patients experiencing inadequate response may increase the dose to $10 \mathrm{mg} / \mathrm{kg}$; or may receive treatment as frequently as every four weeks (Physicians’ Desk Reference, 2002).

The most prescribed dose for adalimumab was $40 \mathrm{mg}$ every other week. However, in patients who had a severe form of the disease, rheumatologists reported administering $40 \mathrm{mg}$ every week. For etanercept, both $25 \mathrm{mg}$ twice every week and 50 mg once a week were being administered. Rheumatologists reported using a range of doses for infliximab from $3 \mathrm{mg} / \mathrm{kg}$ to $10 \mathrm{mg} / \mathrm{kg}$ and these doses were administered every four to eight weeks. A number of studies have shown that there are changes in the dosing of TNF inhibitors especially with infliximab (Gilbert, Smith, \& Ollendorf, 2004; Harley, Frytak, \& Tandon, 2003). Possible explanations for changes in the dosage and dosing interval include adverse events and safety concerns resulting in decreasing in dosages, or poor response to therapy with lower dosages resulting in dosage increases or reduction of dosing intervals (Harley et al., 2003).

There are currently no guidelines available for monitoring patients on TNF 
inhibitors. The only recommended test is the purified protein derivative (PPD), which is done at pretreatment to monitor for tuberculosis. If the PPD test is positive, chest radiography is recommended. The results of this study indicate that rheumatologists are following this recommendation and using PPD monitoring at pretreatment, with chest radiography if the PPD is positive. Results indicate that only $82 \%$ were ordering PPD and $72 \%$ chest radiography, which is recommended if the PPD is positive. Similar results were reported by Yazici and colleagues (2003). Their study reported that 73\% and $83 \%$ rheumatologists ordered PPD tests and $43 \%$ and $50 \%$ ordered chest radiography for etanercept and infliximab, respectively.

There are no guidelines for blood test monitoring, however, a majority of rheumatologists (88-89\%) seemed to order complete blood count (CBC) tests for patients at varying time interval. Percentage of rheumatologists ordering $\mathrm{CBC}$ tests (88-89\%) was lower than that reported by Yazici and colleagues (97-98\%) (2003). Also, creatinine tests (61-63\%) reported by rheumatologists in this study were lower than that reported by Yazici and colleagues (88-89\%) (2003). Rheumatologists who did not order tests such as creatinine and anti-nuclear antibody (ANA) may indicate either a lack of belief on part of the rheumatologists to the utility of these tests or a belief that all patients do not need these tests. In summary, rheumatologists seem to be following similar laboratory monitoring practices for all the three TNF inhibitors but there seems to be no consensus as to how often the tests should be performed. Thus, there is a need to revisit old monitoring guidelines or implement new monitoring guidelines for these TNF inhibitors. 


\section{Results for Objective 5}

The questionnaire asked the respondents to indicate the monitoring guidelines they followed when prescribing the three TNF inhibitors to patients with RA. The responses included ACR guidelines, EULAR guidelines, both ACR and EULAR guidelines, none, and others. Based on the analysis, the null hypothesis $\mathrm{C}$ that rheumatologists are not following any recommended monitoring guidelines for patients with RA on adalimumab, etanercept, and infliximab is rejected. Almost $62 \%$ reported following ACR guidelines and 5\% reported following both ACR and EULAR. Onequarter of the responding rheumatologists reported not following any monitoring guidelines for the TNF inhibitors (Table 43).

\section{Discussion for Objective 5}

Guidelines for monitoring of potential toxicity caused by DMARDs have been developed by American College of Rheumatism (ACR Guidelines) and European League Against Rheumatism (EULAR Guidelines). However, there are no available guidelines for monitoring TNF inhibitors. It is interesting to note that $62 \%$ of the rheumatologists reported following ACR guidelines to monitor patients on TNF inhibitors and around $25 \%$ did not follow any monitoring guidelines. Since there are no established guidelines available for TNF inhibitors, it is possible that rheumatologists who reported following monitoring guidelines continued their practice based on their experiences with earlier DMARDs, particularly MTX (Yazici et al., 2003). 
Table 43. Recommended monitoring guidelines followed by rheumatologists

\begin{tabular}{|c|c|c|c|c|c|}
\hline $\mathrm{TNF}$ & ACR & EULAR & $\begin{array}{c}\text { Both } \\
\text { (ACR \& EULAR) }\end{array}$ & None & Other \\
\hline inhibitors & & $\mathrm{N}(\%)$ & $\mathrm{N}(\%)$ & $\mathrm{N}(\%)$ & $\mathrm{N}(\%)$ \\
\hline Adalimumab & $257(62.2)$ & $1(0.2)$ & $23(5.6)$ & $106(25.7)$ & $26(6.3)$ \\
\hline Etanercept & $261(63.2)$ & $1(0.2)$ & $22(5.4)$ & $106(25.7)$ & $23(5.5)$ \\
\hline Infliximab & $254(61.5)$ & $1(0.2)$ & $22(5.3)$ & $108(26.2)$ & $28(6.8)$ \\
\hline
\end{tabular}

TNF - Tumor necrosis factor, ACR - American College of Rheumatology, EULAR - European League Against Rheumatism 


\section{Results for Objective 6}

Rheumatologists who followed ACR monitoring guidelines were compared to those who reported not following any monitoring guidelines on demographic and practice-related characteristics. Independent $t$-tests and Chi-square tests were conducted for comparisons of these variables. The results of the analysis are presented in Table 44.

Rheumatologists who followed ACR monitoring guidelines were significantly different on age, gender, and number of years in practice compared to rheumatologists who did not follow any monitoring guidelines. Rheumatologists who followed monitoring guidelines were younger and had practiced as a rheumatologist for fewer years as compared to those who did not follow any guidelines. A higher proportion of males followed guidelines more than females.

Rheumatologists who used monitoring guidelines when prescribing each of the TNF inhibitors were compared to those who did not follow any guidelines on perceived barriers to prescribing TNF inhibitors. There was no significant difference between the two groups in all the three TNF inhibitors on perceived barriers to prescribing these agents. 
Table 44. Demographic, practice-related characteristics, and perceived barriers of rheumatologists who followed monitoring guidelines compared to those who do not follow any monitoring guidelines

\begin{tabular}{|c|c|c|c|c|}
\hline Characteristics & $\begin{array}{l}\text { Follow } \\
\text { Guidelines }\end{array}$ & $\begin{array}{l}\text { Don't Follow } \\
\text { Guidelines }\end{array}$ & $\begin{array}{l}\text { Test } \\
\text { Statistic }\end{array}$ & $\begin{array}{l}\text { Significance } \\
\mathrm{P}\end{array}$ \\
\hline Age, years $\left(\right.$ Mean $\_$S.D) & $50.2 \pm 9.1$ & $52.9 \pm 2.6$ & $\mathrm{t}=2.546$ & $0.012^{*}$ \\
\hline Gender, n (\%) & & & $\chi^{2}=7.223$ & $0.007^{*}$ \\
\hline Males & $188(67.1)$ & $92(32.9)$ & & \\
\hline Females & $73(82)$ & $16(18)$ & & \\
\hline Primary Practice Site, n (\%) & & & $\chi^{2}=7.881$ & 0.096 \\
\hline Hospital-based & $9(56.3)$ & $7(43.8)$ & & \\
\hline $\begin{array}{l}\text { University-affiliated } \\
\text { hospital }\end{array}$ & $48(77.4)$ & $14(22.6)$ & & \\
\hline Solo-based & $60(61.9)$ & $37(38.1)$ & & \\
\hline Group-based & $142(74.3)$ & 49 (25.7) & & \\
\hline Other & $2(66.7)$ & $1(33.3)$ & & \\
\hline $\begin{array}{l}\text { Number of years in practice } \\
\text { rheumatologist (Mean } \pm \text { S.D) }\end{array}$ & $\begin{array}{l}\text { as a } \\
17.5 \pm 9.2\end{array}$ & $20.2 \pm 9.8$ & $\mathrm{t}=2.467$ & $0.015^{*}$ \\
\hline $\begin{array}{l}\text { Number of patients with RA } \\
\text { seen per week (Mean } \_ \text {S.D) }\end{array}$ & $30.4 \pm 21.1$ & $30.3 \pm 19.3$ & $t=-0.061$ & 0.951 \\
\hline
\end{tabular}

${ }^{*}$ Significant at $\mathrm{p} \leq 0.05, \mathrm{~S} . \mathrm{D}=$ Standard deviation, $\mathrm{RA}=$ Rheumatoid arthritis 
Table 44 (contd.). Demographic, practice-related characteristics, and perceived barriers of rheumatologists who followed monitoring guidelines compared to those who do not follow any monitoring guidelines

\begin{tabular}{|c|c|c|c|c|c|c|}
\hline \multirow[t]{2}{*}{ Characteristics } & \multicolumn{2}{|c|}{ Adalimumab } & \multicolumn{2}{|c|}{ Etanercept } & \multicolumn{2}{|c|}{ Infliximab } \\
\hline & $\begin{array}{l}\text { Follow } \\
\text { Mean }( \pm S D)\end{array}$ & $\begin{array}{l}\text { Don't follow } \\
\text { Mean }( \pm \mathrm{SD})\end{array}$ & $\begin{array}{l}\text { Follow } \\
\text { Mean }( \pm S D)\end{array}$ & $\begin{array}{l}\text { Don't follow } \\
\text { Mean }( \pm S D)\end{array}$ & $\begin{array}{l}\text { Follow } \\
\text { Mean }( \pm \mathrm{SD})\end{array}$ & $\begin{array}{l}\text { Don't follow } \\
\text { Mean }( \pm S D)\end{array}$ \\
\hline Age of Patient & $2.46( \pm 1.7)$ & $2.13( \pm 1.6)$ & $2.30( \pm 1.6)$ & $2.03( \pm 1.5)$ & $2.64( \pm 1.8)$ & $2.31( \pm 1.6)$ \\
\hline Costs to the Patient & $5.97( \pm 1.5)$ & $6.11( \pm 1.3)$ & $6.02( \pm 1.4)$ & $6.09( \pm 1.3)$ & $5.49( \pm 1.8)$ & $5.37( \pm 1.9)$ \\
\hline $\begin{array}{l}\text { Efficacy of TNF } \\
\text { inhibitors }\end{array}$ & $2.58( \pm 1.5)$ & $2.69( \pm 1.7)$ & $2.31( \pm 1.5)$ & $2.60( \pm 1.7)$ & $2.54( \pm 1.5)$ & $2.80( \pm 1.7)$ \\
\hline $\begin{array}{l}\text { Insurance/Formulary } \\
\text { coverage }\end{array}$ & $5.07( \pm 1.8)$ & $5.00( \pm 1.6)$ & $4.87( \pm 1.9)$ & $4.86( \pm 1.7)$ & $4.62( \pm 2.0)$ & $4.32( \pm 1.9)$ \\
\hline Patient compliance & $2.17( \pm 1.3)$ & $2.34( \pm 1.6)$ & $2.31( \pm 1.4)$ & $2.33( \pm 1.6)$ & $2.27( \pm 1.6)$ & $2.16( \pm 1.5)$ \\
\hline Patient Preference & $2.36( \pm 1.4)$ & $2.53( \pm 1.6)$ & $2.58( \pm 1.4)$ & $2.64( \pm 1.5)$ & $3.05( \pm 1.5)$ & $2.21( \pm 1.6)$ \\
\hline Route of Administration & $2.51( \pm 1.4)$ & $2.60( \pm 1.5)$ & $2.64( \pm 1.4)$ & $2.69( \pm 1.5)$ & $3.58( \pm 1.7)$ & $3.62( \pm 1.7)$ \\
\hline Side Effects & $2.93( \pm 1.4)$ & $3.07( \pm 1.4)$ & $2.73( \pm 1.4)$ & $2.90( \pm 1.3)$ & $3.72( \pm 1.5)$ & $3.88( \pm 1.4)$ \\
\hline Support for Administration & $1.72( \pm 1.3)$ & $1.67( \pm 1.3)$ & $1.73( \pm 1.2)$ & $1.68( \pm 1.3)$ & $2.61( \pm 1.8)$ & $2.76( \pm 1.9)$ \\
\hline
\end{tabular}

$\mathrm{SD}=$ Standard Deviation

Measured on a 7-point scale where $1=$ no problem and $7=$ major problem Independent t-test, significant at $\mathrm{p} \leq 0.05$ 


\section{Discussion for Objective 6}

Based on the analysis, the null hypothesis D that there is no difference in demographic and practice-related characteristics between rheumatologists who follow guidelines and those did not follow any guidelines is rejected. The study results indicated that rheumatologists who followed monitoring guidelines were younger and had fewer years in practice compared to those who did not follow ant guidelines. This is consistent with previous reports that showed that younger physicians are more likely to adhere to guidelines in other disease areas such as hypertension (Fernandes, Madhavan, Amonkar, Dell, Islam, \& Scott, 2003). 


\section{Results for Objective 7}

Figure 7 lists the factors that rheumatologists perceived as problematic regarding the use of specific TNF inhibitors in patients with RA. A repeated measure ANOVA was conducted for comparisons of these variables. The results of the analysis are presented in Table 45 .

There was no perceived difference seen in the patient compliance with these agents $(\mathrm{F}=0.984, \mathrm{p}=0.322)$. Age of the patient $(\mathrm{F}=4.052, \mathrm{p}=0.045)$ was perceived as a minor problem with the perception being the highest for infliximab followed by adalimumab and etanercept. Efficacy of the TNF inhibitors $(\mathrm{F}=4.029, \mathrm{p}=0.049)$ was also perceived as a minor problem with etanercept being considered the most efficacious among the three agents. Costs to the patient $(F=42.612, p=0.000)$ and insurance or formulary coverage $(\mathrm{F}=39.799, \mathrm{p}=0.000)$ were perceived as a major problem with etanercept and adalimumab being perceived as highly expensive and lacking insurance coverage as compared with infliximab.

Patient preference for the TNF inhibitor $(\mathrm{F}=47.343, \mathrm{p}=0.000)$ and preference for route of administration $(\mathrm{F}=73.834, \mathrm{p}=0.000)$ was the highest for adalimumab, followed by etanercept and infliximab. Side effects of TNF inhibitor $(F=112.247, p=$ 0.000 ) was perceived as a major problem for infliximab, followed by adalimumab and least for etanercept. Finally, support for administering infusions $(F=85.209, \mathrm{p}=0.000)$ were perceived more problematic for infliximab than the other two agents. 
Figure 7. Perceived barriers to prescribing TNF inhibitors

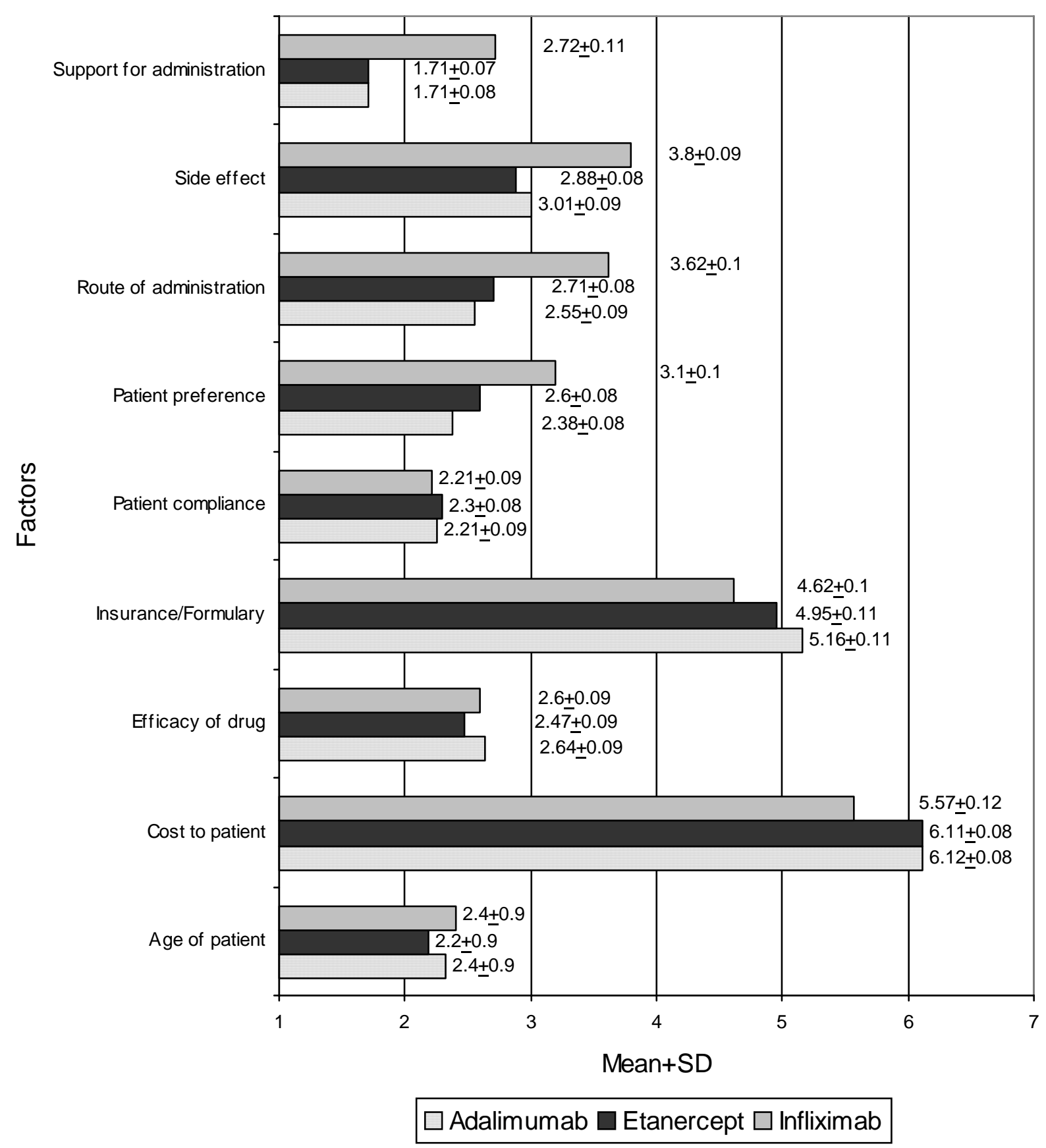

Measured on a 7-point scale, where $1=$ no problem and $7=$ major problem 
Table 45. Perceived barriers to prescribing different TNF inhibitors *

\begin{tabular}{llll}
\hline Factors & $\begin{array}{l}\text { Adalimumab } \\
\text { Mean } \pm \text { SD }\end{array}$ & $\begin{array}{l}\text { Etanercept } \\
\text { Mean } \pm \text { SD }\end{array}$ & $\begin{array}{l}\text { Infliximab } \\
\text { Mean } \pm \text { SD }\end{array}$ \\
\hline Age of patient & $2.32 \pm 0.9^{\mathrm{a}}$ & $2.20 \pm 0.9^{\mathrm{b}}$ & $2.40 \pm 0.9^{\mathrm{a}, \mathrm{b}}$ \\
Costs to the patient & $6.12 \pm 0.8^{\mathrm{c}}$ & $6.11 \pm 0.8^{\mathrm{d}}$ & $5.57 \pm 0.11^{\mathrm{c}, \mathrm{d}}$ \\
$\begin{array}{l}\text { Efficacy of this TNF } \\
\text { inhibitor }\end{array}$ & $2.64 \pm 0.09^{\mathrm{e}}$ & $2.47 \pm 0.09^{\mathrm{e}}$ & $2.60 \pm 0.09$ \\
$\begin{array}{l}\text { Insurance/Formulary } \\
\text { coverage }\end{array}$ & $5.16 \pm 0.10^{\mathrm{f}}$ & $4.95 \pm 0.11^{\mathrm{f}}$ & $4.62 \pm 0.12^{\mathrm{f}}$ \\
$\begin{array}{l}\text { Patient compliance } \\
\text { with this therapy }\end{array}$ & $2.25 \pm 0.08$ & $2.30 \pm 0.08$ & $2.21 \pm 0.09$ \\
$\begin{array}{l}\text { Patient preference for } \\
\text { this TNF inhibitor }\end{array}$ & $2.38 \pm 0.08^{\mathrm{g}}$ & $2.60 \pm 0.08^{\mathrm{g}}$ & $3.10 \pm 0.1^{\mathrm{g}}$ \\
$\begin{array}{l}\text { Route of administration } \\
\text { of this TNF inhibitor }\end{array}$ & $2.55 \pm 0.09^{\mathrm{h}}$ & $2.71 \pm 0.08^{\mathrm{h}}$ & $3.62 \pm 0.1^{\mathrm{h}}$ \\
$\begin{array}{l}\text { Side effects of this } \\
\text { TNF inhibitor }\end{array}$ & $3.01 \pm 0.09^{\mathrm{i}}$ & $2.88 \pm 0.08^{\mathrm{i}}$ & $3.80 \pm 0.09^{\mathrm{i}}$ \\
$\begin{array}{l}\text { Support for administering } \\
\text { Infusions }\end{array}$ & $1.71 \pm 0.08^{\mathrm{j}}$ & $1.71 \pm 0.07^{\mathrm{k}}$ & $2.72 \pm 0.1^{\mathrm{j}, \mathrm{k}}$ \\
\hline
\end{tabular}

* Measured on a 7-point scale where $1=$ no problem and $7=$ major problem $\mathrm{SD}=$ standard deviation

Repeated Measures of Analysis of Variance with post-hoc Tukey comparisons

a,b,c,d,e,f,g,h,l,j,k, Means with same letters are significantly different at $\mathrm{p} \leq 0.05$

$\mathrm{TNF}=$ Tumor necrosis Factor 


\section{Discussion for Objective 7}

Based on the analysis, the null hypothesis E that there are no significant differences in rheumatologists' perception of barriers (insurance coverage, route of administration, patient compliance, side effects, costs to the patient, age of the patient, efficacy of the drugs, and support needed for administration of drugs) to prescribing the three TNF inhibitors is rejected. Two factors (costs to the patient and insurance or formulary coverage) were perceived as major barriers in prescribing the TNF inhibitors. Among the three agents, the perception was a little lower for infliximab. The average wholesale price of these agents for a year of therapy is approximately $\$ 16,000$ (Kavanaugh et al., 2004). Also, as discussed earlier, infliximab, etanercept, and adalimumab have different routes of administration, which has resulted in different coverage and reimbursement policies. Infliximab, which is administered intravenously, is covered under medical benefits while etanercept and adalimumab are administered subcutaneously and are covered only under pharmacy benefits. In addition, due to the tier system of the managed care organizations, patients are required to pay higher copay or sometimes pay the entire cost of etanercept or adalimumab (Gallup, 2001).

Efficacy of TNF inhibitors, side effects of these agents, and age of patient

eligible for these agents were perceived as less problematic. Etanercept was considered as most efficacious with less perceived side effects as compared to adalimumab and infliximab. These TNF inhibitors have been shown to achieve a marked improvement in outcomes in patients with RA and also found to be well tolerated. There are a few side effects reported such as injection-and infusion-site reaction and infections. Largest 
clinical experience among the three agents exists with etanercept since it was the first to be launched in the market. Also, data from clinical trials, practice experience, and postmarketing surveillance reports have shown that etanercept is effective and safe (Fleischmann \& Yocum, 2004).

Rheumatologists perceived patient preference and route of administration as less problematic in prescribing these agents. Adalimumab was perceived to be least problematic in terms of patient preference and route of administration as compared to the other two agents. The patient preference for adalimumab could be high because of the differences in route of administration of these agents. Adalimumab and etanercept are self-administered as subcutaneous injections while infliximab is administered as an infusion. Adalimumab has a dosing schedule of $40 \mathrm{mg}$ every other week as compared to $25 \mathrm{mg}$ twice a week for etanercept and a loading dose at baseline, two, six and every eight weeks for infliximab. This might influence better outcomes in patient with RA although further research is needed to evaluate this possibility.

Finally, support for administering infusions although perceived as a minor problem, was higher for infliximab as compared to the other two agents. Infliximab is administered as an infusion on an outpatient basis and requires the use of professional staff. In addition, it also requires emergency personnel and equipment to monitor any untoward acute infusion reactions. Etanercept and adalimumab, on the other hand, are self-administered by patients and do not require additional staff to administer or monitor patients. 


\section{CHAPTER FIVE}

\section{SUMMARY AND CONCLUSIONS}

This chapter provides a review of the study findings, draws conclusions, presents implications of the study findings, lists limitations of each phase of the study, and provides recommendations for future research.

\section{Phase I}

Tumor necrosis factor (TNF) inhibitors offer important treatment options to patients with RA and are essential to patients that inadequately respond to MTX alone. With the healthcare expenditures rising due to ageing population and technological advances, it is becoming important to assess the "value for money" for these new drugs that are relatively expensive compared to the traditional DMARDs, but then also seem to provide more health benefits to the patients. A few pharmacoeconomic evaluations have been conducted with TNF inhibitors in patients with RA who had an inadequate response to MTX. However, most of the studies have failed to include the withdrawal rates of patients, adverse effects caused by the TNF inhibitors or a decrease in the future risk of disability, thereby potentially biasing the cost-effectiveness ratios. Also, there are no pharmacoeconomic studies that have compared all three TNF inhibitors (adalimumab, etanercept, and infliximab) and leflunomide.

Thus, the goal of this phase of the study was to conduct an incremental costeffectiveness analysis of TNF inhibitors (adalimumab, etanercept, and infliximab) and leflunomide used in combination with MTX in the treatment of patients with RA that 
inadequately respond to MTX alone. In particular, the goal was to quantify the cost per discounted quality-adjusted life year (QALY) gained through the use of etanercept, adalimumab, infliximab, or leflunomide in combination with MTX compared with the standard MTX treatment. The method involved modeling clinical pathways, defining comparators and synthesizing evidence from a range of sources to quantify the model parameters.

\section{Conclusions for Phase I}

The results suggest that the combination of etanercept and MTX should be considered cost-effective when compared against traditional DMARDs (leflunomide plus MTX and standard MTX treatment) and the combination of other two TNF inhibitors with MTX (adalimumab and infliximab) in patients with RA that inadequately respond to MTX alone. The one-way and probabilistic sensitivity analyses suggest that this conclusion is relatively robust to the model assumptions including increased risk of mortality due to RA, consideration of only direct costs, different estimates of indirect costs or exclusion of hospitalization costs. The results also suggest that the combination of leflunomide and MTX is the second most costeffective option in patients with RA that inadequately respond to MTX alone. This treatment was the most cost-effective when the indirect costs were valued at three times that of the direct costs. In a base-case analysis, adalimumab plus MTX and infliximab plus MTX cost more and had a higher ICER than leflunomide plus MTX. Therefore, these options were not cost-effective. 
Etanercept plus MTX provided more health benefits (15.84 QALYs) and was more costly than standard MTX treatment, with resulting cost-effectiveness ratios of \$49,724/QALY. The second most cost-effective treatment was leflunomide plus MTX with an ICER of \$52,833/QALY. The lowest cost/QALY for etanercept plus MTX and leflunomide plus MTX were \$30,371/QALY and \$43,720/QALY, respectively. The highest cost/QALY values for etanercept plus MTX and leflunomide plus MTX were \$74,628/QALY and \$63,126/QALY, respectively. These ICERs for both the treatments remained within the acceptable range of $\$ 50,000$ and $\$ 100,000$ as compared to other comparators.

\section{Phase II}

Even though infliximab, etanercept, and adalimumab belong to the same class of drugs, they have distinct clinical, pharmacokinetic, and pharmacodynamic properties that must be considered when selecting any one of them for therapy. These drugs have the potential to reduce future disability and cause remission in RA; however, the high acquisition cost of these agents coupled with intensive monitoring required for toxicities seem to be the treatment-limiting factors. Since the TNF inhibitors have recently been introduced in the market, there is not enough data available regarding the use of these drugs by rheumatologists. Very little information is available regarding rheumatologists practice patterns, laboratory monitoring practices, and barriers they perceive in prescribing these agents. This information can only be clarified with the availability of data from evaluation of physician practice experiences. Thus, the goal of 
phase II of the study was to survey a national sample of rheumatologists and assess their practice patterns with respect to the use of these three agents.

\section{Conclusions for Phase II}

The conclusions of this phase of the study are presented based on the objectives proposed in the study.

Objective 1: Prescribing patterns for the three TNF inhibitors: adalimumab, etanercept, and infliximab.

As expected, rheumatologists were prescribing the TNF inhibitors in patients with moderate to severe RA. An important finding was that some rheumatologists had broadened the official indication of these agents to patients with early-onset RA. In patients with severe RA that inadequately responded to MTX alone, rheumatologists reported using a combination of TNF inhibitor with a traditional DMARD indicating a preference for combination treatments in patients with severe disease. Another finding was that a majority of rheumatologists reported switching patients from one TNF inhibitor to another TNF inhibitor due to lack of efficacy or adverse events of the first one.

Objective 2: Rheumatologists' preference for a particular TNF inhibitor.

The null hypothesis aimed to investigate that there is no preference for any particular TNF inhibitor. The finding of the survey showed a preference for etanercept followed by adalimumab and infliximab. 
Objective 3: Rheumatologist characteristics and perceived barriers in prescribing TNF inhibitors and preference for a particular TNF inhibitor.

The null hypothesis aimed to investigate that there is no relationship of preference for a particular TNF inhibitor and demographic, practice-related characteristics, and perceived barriers in prescribing TNF inhibitors. The study revealed that male rheumatologists preferred these agents to females. Also, rheumatologists who preferred infliximab saw more patients per week compared to other two TNF inhibitors. Rheumatologists who preferred infliximab perceived the need for additional support for administration of infliximab compared with etanercept and adalimumab. Rheumatologists who preferred etanercept perceived patient compliance as a barrier compared to those who preferred adalimumab. However, both perceptions were considered as minor problems.

Objective 4: Laboratory monitoring practices and the frequency of tests ordered for patients on TNF inhibitors.

The complete blood count tests and the purified protein derivative tests were ordered by a majority of the rheumatologists for all three TNF inhibitors. The antinuclear antibody tests and creatinine tests were ordered by only one-third to half of the rheumatologists. Also, there was a wide variation in terms of frequency at which these tests were ordered. The most commonly used doses for adalimumab and etanercept were $40 \mathrm{mg}$ every other week and $25 \mathrm{mg}$ twice every week, respectively. A range of doses and dosing intervals were reported with the use of infliximab. 
Objective 5: Recommended monitoring guidelines for patients with RA on adalimumab, etanercept, and infliximab.

A majority of rheumatologists reported following ACR monitoring guidelines. Since there are no established monitoring guidelines for TNF inhibitors, it is believed that rheumatologists were using guidelines based on their past experiences with traditional DMARDs. Another finding was that one-fourth of the rheumatologists did not follow any monitoring guidelines for patients who used TNF inhibitors.

Objective 6: Rheumatologist characteristics and perceived barriers in prescribing TNF inhibitors and monitoring guidelines.

The null hypothesis aimed to investigate that there is no relationship of adherence to monitoring guidelines and demographic, practice-related characteristics, and perceived barriers in prescribing TNF inhibitors. Rheumatologists who followed monitoring guidelines were a little younger and had fewer years of practice. Although the results were significantly different indicating a difference in age and years of practice between those who follow guidelines and those who don't, it only remains to be seen if a difference of two years is relevant in clinical practice.

Objective 7: Perceived barriers in prescribing TNF inhibitors.

Costs to patients and insurance coverage were perceived as major problems and patient preference, route of administration, and side effects were perceived as minor problems. Among the three TNF inhibitors, the perception for costs to patients and insurance coverage was a little lower for infliximab compared to etanercept and 
adalimumab. For factors like patient preference, route of administration, and side effects, the perception was lower for infliximab compared to etanercept and adalimumab.

\section{Implications of Study Findings}

The study was undertaken to assess the cost-effectiveness of TNF inhibitors in patients that inadequately respond to MTX alone. The study also aimed to assess the prescribing patterns and laboratory monitoring practices of rheumatologists using TNF inhibitors. The study findings should be useful to decision makers such as payers and prescribers.

\section{Implications to the Payers}

The study results indicate that the combination of etanercept and MTX is the most cost-effective option in patients with RA that inadequately respond to MTX alone. Also, the survey results indicate that rheumatologists prefer etanercept to infliximab and adalimumab for a variety of reasons, including method of administration and adverse-events profile. Patients with greater disease severity incur high costs and these new therapies can have long-term savings by preventing functional decline and subsequent hospitalization and surgery in these patients. Even though the long-term impact of these agents on joint damage cannot be determined with certainty at present, a reduced risk of surgery will reduce the demand for orthopedic services. However, the disparity in reimbursement based on different routes of administration for these medications underscores the need for investing in long-term positive outcomes through 
aggressive treatment of RA. Managed care organizations need to consider these issues when making formulary and coverage decisions.

The study findings also indicate that there are no established treatment guidelines for these agents. Rheumatologists are prescribing these agents to patients in mild RA and also switching patients from one TNF inhibitor to another without much evidence available regarding the usefulness of this practice. The practice of switching may be effective but if not, it may be a waste of time and money. Thus, there is a need to develop strategies to increase the appropriate prescribing and monitoring of these agents till sound evidence are available supporting the usefulness of switching TNF inhibitors or using these agents in patients with early forms of the disease.

\section{Implications to the Prescribers}

The use of TNF inhibitors to treat RA has implications for current practices in rheumatology. The widespread use of etanercept and adalimumab would place a greater demand on outpatient services. This would include increased involvement of health care providers like nurses to teach patients to self-administer injections and to provide disease and drug monitoring services. The widespread use of infliximab will lead to greater utilization of rheumatology facilities and support staff. Currently there are no monitoring guidelines available and there is a wide variation in laboratory monitoring practices among rheumatologists. Thus, interested practitioners, manufacturers, and policymakers should revisit old monitoring guidelines or perhaps implement new monitoring guidelines for the use of these newer agents. 


\section{Limitations of the Study}

Both phases of the study have some limitations and these are discussed in the following section. These limitations need to be considered when deriving inferences from the reported results.

\section{Limitations of Phase I}

1. The main limitation relates to available data. An ideal source would have been a very long-term, randomized study with a large sample size examining the efficacy and resources utilized by all the therapeutic options in patients with RA that inadequately respond to MTX alone. In the absence of such a study, evidence had to be assembled from a range of sources and these sources have their advantages and disadvantages from a health economic perspective. Thus, the results of any modeling study need to be treated with some degree of caution.

2. The base-case analysis in the decision analytic model assumes that the baseline characteristics of the study patients across RCTs are comparable based on their close similarities in important baseline demographic and clinical characteristics. It is a general view that absolute comparability across different treatments can be established through randomization. Currently, such data does not exist and it is doubtful whether all the treatment options considered in the analysis would be evaluated using a single head-to-head randomized controlled clinical trial in the near future. 
3. The efficacy data for each treatment option except for adalimumab plus MTX and standard MTX treatment comes from a single respective RCT. Therefore, there will be generalizability issues with the base-case estimates used in the Phase I of the study. However, sensitivity analyses were conducted to address this limitation and the results obtained seemed to be robust at different values of input parameters.

4. The three TNF inhibitors and leflunomide have different modes of administration. Since no data is available indicating differing rates of compliance among the different TNF inhibitors and leflunomide in patients with RA, the potential impact of compliance was not considered in the model. The model assumed 100\% compliance. This assumption was necessary to fully quantify the costs and consequences of introducing these agents under ideal conditions. However, it would be important to see whether the ICERs change when compliance is introduced in the model.

5. It was assumed that patients who did not respond to the treatments were switched to palliative care. Currently, there is no data to show the actual distribution of time a patient spends on each of these drugs. Also, in actual clinical settings, if the patient fails to respond to one agent, rheumatologists may switch the patient to a different DMARD or a TNF inhibitor. Thus, a sequential use of DMARDs could have been a better comparator. Given the complexity of 
treatment regimens, it is very difficult to decide on the sequences of DMARDs and also the placement of TNF inhibitors in these regimens.

\section{Limitations of Phase II}

1. This phase of the study was a mail survey and therefore all the limitations of a mail survey will be inherent in this study. Some of these limitations include measurement errors due to respondents not understanding the instructions or items, and recall bias, which can affect the accuracy of inferences drawn from the survey responses.

2. Even though the survey instrument has face and content validity, it has not been otherwise validated.

3. The nonresponse bias analysis revealed differences in some variables such as age and years in practice between respondents and nonrespondents. Caution should, therefore, be exercised when extrapolating the findings to the entire rheumatologist population.

\section{Recommendations for Future Research}

The model presented in the study was populated using different data sources and each of the sources had their own strengths and limitations. The majority of data came from clinical trials which are conducted under controlled conditions and therefore do not reflect the real clinical setting. Future studies should try and incorporate data in 
the model from observational study. For example, various TNF registries have been established all over world. It is too early to get such a data from the registry however, in the future; these might provide refinement of estimates such as response rates, withdrawal rates, and long-term disability progression. These data can also be useful in estimating the compliance of different drugs. Other priorities for future research should include estimating costs associated with RA incurred by patients and their families, including a fuller coverage of adverse events of TNF inhibitors and other traditional DMARDs.

Strategies for treating RA are potentially very complex and could include combination treatments, triple therapies, or a sequence of drugs. A number of events such as joint replacement, hospitalization, switching due to lack of efficacy or adverse events take place in a patient's lifetime. An advanced modeling technique such as discrete event simulation can be used to represent such structure and produce a realistic set of virtual patient histories thereby giving a more accurate estimation. 


\section{BIBLIOGRAPHY}

American College of Rheumatology (ACR) Subcommittee on Rheumatoid Arthritis Guidelines. (2002). Guidelines for the management of Rheumatoid Arthritis: 2002 Update. Arthritis Rheum.,46 (2),328-346.

Albert, D.A., Aksentijevich, S., Hurst, S., Fries, J.F., \& Wolfe, F. (2000). Modeling therapeutic strategies in rheumatoid arthritis: Use of decision analysis and Markov model. J Rheumatol, 27,644-52.

Allaire, S.H., Prashker, M.J., \& Meenan, R.F. (1994). The costs of rheumatoid arthritis. Pharmacoeconomics, 6 ,513-522.

Bansback, N., Brennan, A., \& Ghatnekar, O. (2004). The cost-effectiveness of adalimumab in the treatment of moderate to severe rheumatoid arthritis patients in Sweden. Ann Rheum Dis; Published Online First [date of publication]. Doi:10.1136/ard.2004.027565.

Bansback, N. J., Regier, D. A., Ara, R., Brennan, A., Shojania, K., Esdaile, J. M. et al. (2005). An overview of economic evaluations for drugs used in rheumatoid arthritis: focus on tumour necrosis factor-alpha antagonists. Drugs, 65, 473-496.

Barrett, E.M., Scott, D.G., Wiles, N.J., Symmons, D.P. (2000). The impact of rheumatoid arthritis on employment status in the early years of disease: a UK community based study. Rheumatology, 39,1403-9.

Barton, P., Jobanputra, P., Wilson, J., Bryan, S., \& Burls, A. (2004). The use of modeling to evaluate new drugs for patients with a chronic condition: the case of antibodies against tumor necrosis factor in rheumatoid arthritis. Health Technol Assess, 8, (11), iii,1-91.

Blumenauer, B., Coyle, D., \& Tugwell, P. (2002). Pharmacoeconomics of long-term treatment of rheumatoid arthritis. Expert.Opin.Pharmacother., 3, 417-422.

Brennan, A. \& Akehurst, R. (2000). Modelling in Health Economic Evaluation. What is its place? What is its value? Pharmacoeconomics, 17: 445-459.

Brennan, A., Bansback, N., Conway, P., et al. (2001). Modelling the cost-effectiveness of etanercept in adults with rheumatoid arthritis in the UK. Arthritis Rheum, 44, Suppl. 9, 157.

Brennan, A., Bansback, N., Reynolds, A., \& Conway, P. (2003). Modelling the costeffectiveness of etanercept in adults with rheumatoid arthritis in the UK.

Rheumatology, 42,1-13.

Briggs, A. \& Sculpher, M. (1998). An introduction to Markov modelling for economic evaluation. Pharmacoeconomics, 13, 397-409. 
Cairns, A.P., \& Taggart, A.J. (2002). Anti-tumor necrosis factor therapy for severe inflammatory arthritis: Two years experience in Northern Ireland. Ulster Med J., 71, (2), 101-105.

Callahan, L. F. (1998). The burden of rheumatoid arthritis: facts and figures. $J$ Rheumatol Suppl, 53, 8-12.

Centers for Medicare and Medicaid services. Available at:

http://www.cms.hhs.gov/providers/pufdownload/default.asp?. Accessed on February 14, 2005

Chiou, C. F., Wanke, L. K., Reyes, C, et al. (2003). A cost-efficacy comparison of biologic agents in the treatment of rheumatoid arthritis. Ann Rheum Dis., 62, Suppl. 1, 353.

Choi, H. K., Seeger, J. D., \& Kuntz, K. M. (2002). A cost effectiveness analysis of treatment options for methotrexate-naive rheumatoid arthritis. J Rheumatol, 29, 11561165.

Choi, H. K., Seeger, J. D., \& Kuntz, K. M. (2000). A cost-effectiveness analysis of treatment options for patients with methotrexate-resistant rheumatoid arthritis. Arthritis Rheum., 43, 2316-2327.

Choy, E. H. (2004). Two is better than one? Combination therapy in rheumatoid arthritis. Rheumatology, 43,1205-1207.

Cimino, W.G., \& O'Malley, M. J. (1998). Rheumatoid arthritis of the ankle and hindfoot. Rheum Dis Clin North Am., 24,157-172.

Data TreeAge Software Inc. (2002). Healthcare user's manual. Massachussetts.

DeLeeuw, E. (1992). Data quality in mail, telephone, face to face surveys. Amsterdam: TT Publikaties.

Dillman, D. A. (1999). Mail and internet surveys-the tailored design method. $2^{\text {nd }}$ ed. New York: Wiley and Sons.

Doyle, J. J. (2001). Economic and quality of life impact of rheumatoid arthritis. Managed Care, 10, (7), 15-18

Drug Topics Red Book. (2004). Fleming, T. (Ed). $108^{\text {th }}$ ed., Medical Economics Company, Montvale, NJ.

Drummond, M. F., O’Brien, B., Stoddart, G. L., \& Torrance, G. W. (1998). Methods for the economic evaluation of health care programmes. $2^{\text {nd }}$ ed., Oxford, United Kingdom: Oxford University Press. 
Emery, P. (2004). Review of health economics modelling in rheumatoid arthritis. Pharmacoeconomics, 22, 55-69.

Erkan, D., Yazici, Y., Harrison, M. J., \& Paget, S. A. (2002). Physician Treatment preferences in rheumatoid arthritis of differing disease severity and activity: The impact of cost on First-Line therapy. Arthritis Rheum., 47, (3),285-290.

Fernandes, A., Madhavan, S., Amonkar, M., Dell, D., Islam, S., \& Scott, V. (2003). Evaluating utilization of beta-blockers as secondary prevention for post myocardial infarction in a Medicaid population. (Doctoral dissertation, West Virginia University, 2003)

Fleischmann, R. \& Yocum, D. (2004). Does safety make a difference in selecting the right TNF antagonist? Arthritis Res. Ther., 6 Suppl 2, S12-S18.

Flendrie, M., Creemers, M. C., Welsing, P. M., den Broeder, A. A., \& van Riel, P. L. (2003). Survival during treatment with tumor necrosis factor blocking agents in rheumatoid arthritis. Ann Rheum Dis., 62, ii, 30-3.

Gabriel, S. E. (2001). The epidemiology of Rheumatoid Arthritis. Rheum Dis Clin North Am., 27, (2), 269-281.

Gabriel, S., Tugwell, P., O’Brien, B., Yelin, E., Drummond, M., Ruff, B., et. al. (1999). Report of the OMERACT Task Force on Economic Evaluation. Outcome Measures in Rheumatology. J Rheumatol., 27, 203-206.

Gallup, E. (2001). Coverage inequalities of new therapies for rheumatoid arthritis in a managed care setting. Manag Care Interface,52-58, 69.

Genovese, M. C., Bathon, J. M., Martin, R.W. (2002). Etanercept versus methotrexate in patients with early rheumatoid arthritis: a two-year radiographic and clinical outcomes. Arthritis Rheum., 46,1443-50.

Gilbert, T. D., Smith, D., \& Ollendorf, D.A. (2004). Patterns of use, dosing, and economic impact of biologic agent use in patients with rheumatoid arthritis: a retrospective cohort study. BMC Musculoskelet Disord., 5, 36.

Gold, M. R., Russell, L. B., Siegel, J. E., \& Weinstein M. C. (1996). Cost-effectiveness in health and medicine. New York: Oxford University Press.

Griffiths, R.I., Bar-Din, M., MacLean, C., Sullivan, E. M., Herbert, R. J., Yelin, E. H. (2001). Patterns of disease modifying antirheumatic drug use, medical resources consumption, and cost among rheumatoid arthritis patients. Ther Apher., 5, (2),92-104.

Guedes, C. (1999). Mortality in rheumatoid arthritis. Rev Rheum Engl Ed, 66,1895-9. 
Haraoui, B. (2004). Is there a rationale for switching from one anti-tumor necrosis factor agent to another? J Rheumatol, 31, 1021-22.

Harley, C.R., Frytak, J. R., \& Tandon, N. (2003). Treatment compliance and dosage administration among rheumatoid arthritis patients receiving infliximab, etanercept, or methotrexate. Am J Manag Care, 9, 6, S136-S143.

Hochberg, M.C., Tracy, J.K., Holt-Hawkins, M., \& Flores, R.H. (2003). Comparison of efficacy of the tumor necrosis factor $\alpha$ blocking agents adalimumab, etanercept, and infliximab when added to methotrexate in patients with active rheumatoid arthritis. Ann Rheum Dis., 62, Suppl II, ii 13-iil6.

Homik, J. E., \& Suarez-Alamor, M. (2004). An economic approach to health care. Best Pract Res. Clin Rheumatol.,18, (2),203-218.

Jobanputra, P., Barton, P., Bryan, S., Burls, A. (2002). The effectiveness of infliximab and etanercept for the treatment of rheumatoid arthritis: a systematic review and economic evaluation. Health Technol Assess,6, (21),1-110.

Jobanputra, P., Wilson, J., Douglas, K., \& Burls, A. (2004). A survey of British rheumatologists' DMARD preferences for rheumatoid arthritis. Rheumatology, 43,206210.

Kalton, G. (Ed.). (1987). Introduction to survey sampling. Sage University Paper series on Quantitative Application in Social Sciences, Series/Number 07-035, Beverly Hills and London, Sage Pubns.

Kavanaugh, A., Cohen, S., \& Cush, J. J. (2004). The evolving use of tumor necrosis factor inhibitors in rheumatoid arthritis. J Rheumatol, 31, 1881-1884.

Kavanaugh, A., Heudebert, G., Cush, J., \& Jain, R. (1996). Cost valuation of novel therapeutics in rheumatoid arthritis (CENTRA): A decision analysis model. Semin Arthritis Rheum., 25, (5), 297-307.

Kleinbaum, D. G., Kupper, L. L. \& Morgenstern, H. (1982). Epidemiologic research: Principles and quantitative methods: New York: Van Nostrand Reinhold

Kobelt, G., Jonsson, L., Lindgren, P., Young, A., \& Eberhardt, K. (2002). Modeling the progression of Rheumatoid Arthritis. Arthritis Rheum., 46, (9), 2310-19.

Kobelt, G., Jonsson, L., Young, A., \& Eberhardt, K. (2003). The cost effectiveness of infliximab (Remicade ${ }^{\circledR}$ ) in the treatment of rheumatoid arthritis in Sweden and the UK based on the ATTRACT study. Rheumatology, 42, 326-335.

Kobelt, G., Lindgren, P., \& Young, A. (2002). Modelling the costs and effects of leflunomide in rheumatoid arthritis. Eur J Health Econ., 3, 180-187. 
Kremer, J.M. (2001). Rational use of new and existing disease modifying agents in Rheumatoid Arthritis. Annals of Intern Med, 134, 695-706.

Kremer, J.M, Genovese, M., Cannon, G.W., Caldwell, J., Cush, J., Furst, D.E. et al. (2004). Combination of leflunomide and methotrexate (MTX) therapy for patients with active rheumatoid arthritis failing MTX monotherapy: Open-label extension of a randomized, double-blind, placebo-controlled trial. J Rheumatol., 31, 1521-1531.

Kremer, J.M, Genovese, M., Cannon, G.W., Caldwell, J., Cush, J., Furst, D.E. et al. (2002). Concomitant leflunomide therapy in patients with active rheumatoid arthritis despite stable doses of methotrexate. Ann Intern Med.,137, 726-733.

Keystone, E. C., Kavanaugh, A. F., Sharp, J. T., Tannenbaum, H., Hua, Y., Teoh, L. S., et al. (2004). Radiographic, clinical, and functional outcomes of treatment with adalimumab (a human Anti-Tumor Necrosis Factor Monoclonal Antibody) in patients with active Rheumatoid arthritis receiving concomitant methotrexate therapy. Arthritis Rheum.,50, (5), 400-1411.

Klareskog, L., van der Heijde, D., de Jager, J. P., Gough, A., Kalden, J., Malaise, M. et al. (2004). Therapeutic effect of the combination of etanercept and methotrexate compared with each treatment alone in patients with rheumatoid arthritis: double-blind randomized controlled trial. Lancet, 363, 675-81.

Lan, J., Chou, S., Chen, D., Chen, Y., Hsieh, T., Young, M. (2004). A comparative study of etanercept plus methotrexate and methotrexate alone in Taiwanese patients with active rheumatoid arthritis: A 12-week, double-blind, randomized, placebocontrolled study. J Formos Med Asson, 103,618-213.

Lipsky, P. E., van der Heijde, D., Clair, E.W., Furst, D.E., Breedveld, F.C., Kalden, J.R., et al. (2000). Infliximab and methotrexate in the treatment of rheumatoid arthritis. New Eng J Med.,343, 1594-602.

Lipsky, P. E., \& Kavanaugh, A. (1999). The impact of pharmaco-economic considerations on the utilization of novel anti-rheumatic therapies. Rheumatology, 38, Suppl. 2: 41-4.

Louie, S. G., Park, B., \& Yoon, H. (2003). Biological Response Modifiers in the management of rheumatoid arthritis. Am J Health Syst Pharm. 60, 346-55.

Maetzel, A. (2004). Defining therapeutic success in rheumatoid arthritis clinical trials: from statistical significance to clinical significance. J Rheumatol, 31, 411-412.

Maetzel, A., Bombardier, C., Strand, V., Tugwell, P., \& Wells, G. (1998). How Canadian and US Rheumatologists treat moderate or aggressive Rheumatoid Arthritis: A Survey. J Rheumatol., 25, 2331-8. 
Maini, R., St. Claire, E. W., Breedveld, F., Furst, D., Kalden, J., Weisman, M. et al. (1999). For the ATTRACT study group. Infliximab (chimeric anti-tumor necrosis factor alpha monoclonal antibody) versus placebo in rheumatoid arthritis patients receiving concomitant methotrexate: a randomized Phase III trial. Lancet, 354, 1932-9.

Malone, D. C., Singh, A., Wanke, L. A., et al. (2003). A cost-efficacy comparison of adalimumab and etanercept in the treatment of rheumatoid arthritis. Ann Rheum Dis., 62, Suppl. 1, 537.

Managed Care Lab Fee Schedule. Available at: http://www.purdue.edu/hr/Benefits/mac.htm. Accessed on February 20, 2005

Meenan, R. F., Yelin, E. H., Henke, C. J., Curtis, D. L., Epstein, W. V. (1978). The costs of rheumatoid arthritis. A patient oriented study of chronic disease costs. Arthritis Rheum., 21, 827-833.

Michaud, K., Messer, J., Choi, H. K., \& Wolfe, F. (2003). Direct medical costs and their predictors in patients with rheumatoid arthritis: a three-year study of 7,527 patients. Arthritis Rheum., 48, 2750-2762.

Miller, D., \& Homan, S. (1994). Determining transition probabilities. Med Decis Making, 14, 52-58.

Moots, R., Taggart, A., \& Walker, D. (2003). Biologic therapy in clinical practice: enthusiasm must be tempered by caution. Rheumatology, 42, 614-616.

Moreland, L.W., Cohen, S.B., Baumgartner, S.W., Tindall, E.A., Bulpitt, K., Martin, R., et al. (2001). Long-term safety and efficacy of etanercept in patients with rheumatoid arthritis. J Rheumatol, 28, 1238-44.

Muennig, P. (2002). Designing and conducting cost-effectiveness analyses in medicine and health care. $2^{\text {nd }}$ ed., John Wiley $\&$ Sons, Inc.

Nuijten, M. J., Engelfriet, P., Duijn, K., Bruijn, G., Wierz, D., \& Koopmanschap, M. (2001). A cost-cost study comparing etanercept with infliximab in rheumatoid arthritis. Pharmacoeconomics, 19, 1051-64.

Physicians' Desk Reference. (2005). Murray, L. (Ed.). Medical Economics Company, Inc. Montvale, NJ.

Pope, J. E., Hong, P., \& Koehler, B. E. (2002). Prescribing trends in Disease Modifying Antirheumatic drugs for Rheumatoid Arthritis: A survey of Canadian Rheumatologists. J Rheumatol., 29, 255-60.

Pinals, R. S. (1987). Survival in rheumatoid arthritis. Arthritis Rheum., 30, 473-5. 
Pugner, K. M., Scott, D. I., Holmes, J. W., \& Hieke, K. (2000). The costs of rheumatoid arthritis: an international long-term view. Semin Arthritis Rheum., 29, $305-$ 20 .

Ruff, B. (1999). OMERACT: Economic evaluations and health policy. J Rheumatol., 26, 2076-7.

Schnabel, A. (2004). Disease modifying Antirheumatic drugs: enhancing efficacy by combination. Lancet, 363, 670-671.

Schwartzman, S., Fleischmann, R., \& Morgan, G. J. (2004). Do anti-TNF agents have equal efficacy in patients with rheumatoid arthritis? Arthritis Res Ther, 6, (suppl 2), S3S11.

Schwartzman, S., \& Morgan, G. J. (2004). Does route of administration affect the outcome of TNF antagonist therapy? Arthritis Res Ther, 6, Suppl 2, S19-S23.

Seymour, H. E., Worsley, A., Smith, J. M., \& Thomas, S. H. L. (2001). Anti-TNF agents for rheumatoid arthritis. Br J Clin Pharmacol., 51, 201:208.

Shaw, J. W., \& Zachary, W. M. (2002). Applications of probabilistic sensitivity analysis in decision analytic modeling. Formulary, 37, 32-40.

Silman, A. J., \& Pearson, J. E. (2002). Epidemiology and genetics of rheumatoid arthritis. Arthritis Res., 4, Suppl 3, S265-S272.

Smolen, J., ven der Heijde, D., Emery, P., et al. (2004). Infliximab inhibits radiographic joint damage in patients with early rheumatoid arthritis regardless of clinical response after 54-week treatment. Ann Rheum Dis, 63, Suppl. I, 260.

Sonnenberg, F. A., \& Beck, J. R. (1993). Markov models in medical decision-making: a practical guide. Med Decis Making, 13, 322-38.

Stone, C. E. (1984). The lifetime economic costs of rheumatoid arthritis. J Rheumatol., $11,819-827$.

Strand, V., Cohen, S., Schiff, M., Weaver, A., Fleischmann, R., Cannon, G. et al. (1999). Treatment of active rheumatoid arthritis with leflunomide compared with placebo and methotrexate. Arch Intern Med, 159, 2542-2550.

Terebelo, S. (2003). Rheumatoid arthritis: making the diagnosis - Board review. Clin Rev., 13 (2), 61

Toussirot, E., \& Wendling, D. (2004). The use of TNF- $\alpha$ blocking agents in rheumatoid arthritis: an overview. Expert Opin Pharmacother, 5, (3), 581-594. 
Tugwell P. (2000). Pharmacoeconomics of drug therapy for rheumatoid arthritis. Rheumatology, 39, (suppl. 1), 43-47.

U.S. Census Bureau (Vital Statistics), the Official Statistics, Statistical Abstract of the United States: 2002.

U.S. Department of Labor. Bureau of Labor Statistics. (http://stats.bls.gov). Accessed on January, 14, 2005.

van Vollenhoven, R.F. (2004). Switching between biologicals. Clin Exp Rheumatol., 22, Suppl. 35, S115-121.

van Vollenhoven, R.F., Ernestam, S., Harju, A., Bratt, J., \& Klareskog, L. (2003). Etanercept versus etanercept plus methotrexate: a registry-based study suggesting that the combination is clinically more efficacious. Arthritis Res Ther., 5, R347-R351.

Weinblatt, M. E., Keystone, E. C., Furst, D. E., Moreland, L. W., Weisman, M. H., Birbara, C. A., et al. (2003). Adalimumab, a fully human anti tumor necrosis factor $\alpha$ monoclonal antibody, for the treatment of rheumatoid arthritis in patients taking concomitant methotrexate. Arthritis Rheum., 48, (1), 35-45.

Weinblatt, M. E., Kremer, J. M., Bankhurst, A. D., Bulpitt, K. J., Fleischmann, R. M., Fox, R. I., et al. (1999). A trial of etanercept, a recombinant tumor necrosis factor receptor: Fc fusion protein, in patients with rheumatoid arthritis receiving methotrexate. $N$ Eng J Med, 340, 253-259.

Weinblatt, M. E., Kremer, J. M., Coblyn, J. S., Maier, A. L., Helfgott, S. M., Morrell, M. et al. (1999). Pharmacokinetics, safety, and efficacy of combination treatment with methotrexate and leflunomide in patients with active rheumatoid arthritis. Arthritis Rheum., 42, 1322-1328.

Welsing, P. M., Severens, J. L., Hartman, M., van Riel, P. L., \& Laan, R. F. (2004). Modeling the 5-year cost effectiveness of treatment strategies including tumor necrosis factor-blocking agents and leflunomide for treating rheumatoid arthritis in the Netherlands. Arthritis Rheum., 51, 964-973.

Wolfe, F., Albert, D. A., \& Pincus, T. (1998). A survey of United States Rheumatologists concerning effectiveness of Disease Modifying Antirheumatic Drugs and Prednisone in the treatment of Rheumatoid Arthritis. Arthritis Care Res., 11, (5), 375-380.

Wolfe, F., Mitchel, D. M., Sibley, J.T., Fries, J. F., Bloch, D. A., Williams, C. A., et al. (1994). The mortality of rheumatoid arthritis. Arthritis Rheum.,37, 481-94.

Wolfe, F., Rehman, Q., Lane, N. E., \& Kremer, J. (2001). Starting a disease modifying antirheumatic drug or biologic agent in Rheumatoid arthritis: Standards of practice for RA treatment. J Rheumatol., 28, 1704-11. 
Wolfe, F., \& Zwillich, S. (1998). The long-term outcomes of rheumatoid arthritis. A 23-year prospective longitudinal study of total joint replacement and its predictors in 1,600 patients with rheumatoid arthritis. Arthritis Rheum., 41, 1072-82.

Wong, J. B., Ramey, D. R., \& Singh, G. (2001). Long-term morbidity, mortality, and economics of rheumatoid arthritis. Arthritis Rheum., 44, (12), 2746-2749.

Wong, J. B., Singh, G., \& Kavanaugh, A. (2002). Estimating cost-effectiveness of 54 weeks of Infliximab for Rheumatoid Arthritis. Am J Med., 113, 400-408.

Wong, J.B. (2004). Cost-effectiveness of anti-tumor necrosis factor agents. Clin Exp Rheumatol., 22, Suppl. 35, S65-S70.

Yazici, Y. \& Erkan, D. (2004). Do etanercept-naive patients with rheumatoid arthritis respond better to infliximab than patients for whom etanercept has failed? Ann Rheum Dis., 63, 607-608.

Yazici, Y., Erkan, D., \& Paget, S. A. (2003). Monitoring by rheumatologists for Methotrexate-, Etanercept-, Infliximab-, and Anakinra-associated adverse events. Arthritis Rheum., 48, (10), 2769-2772.

Yelin, E., \& Wanke, L. A. (1999). An assessment of the annual and long-term direct costs of rheumatoid arthritis: the impact of poor function and functional decline. Arthritis Rheum, 42, 1209-1218.

Zentilin, P., Seriolo, B., Dulbecco, P., Caratto, E., Iiritano, E., Fasciolo, D., et al. (2002). Eradication of Helicobacter pylori may reduce disease severity in rheumatoid arthritis. Aliment Pharmacol Ther, 16 ,1291-1299. 


\section{APPENDIX A: FIRST COVER LETTER}

Date: October 10, 2004

Dear Physician:

We are writing to seek your opinions on an important class of drugs that you use in your practice. Tumor Necrosis Factor (TNF) inhibitors such as adalimumab, etanercept, and infliximab, have advanced the treatment of Rheumatoid Arthritis. Clinical trials with these drugs have shown that they may be more effective than traditional agents because of their ability to prevent or control joint damage as well as diminish pain. However, these drugs have been only recently introduced in the market, and there are numerous questions regarding their appropriate dosage, monitoring schedules, and target population.

The objective of the current research study is to assess the current treatment practices of rheumatologists who use TNF inhibitors in patients with Rheumatoid Arthritis. This research study is part of a doctoral (Ph.D.) dissertation project and is being undertaken and funded by the West Virginia University School of Pharmacy. Information obtained from this research study will be useful in determining common practices and issues associated with the use of TNF inhibitors.

Your name was randomly selected from a national list of rheumatologists. We hope that you will participate by completing the attached questionnaire that asks about your current treatment practices and experiences using TNF inhibitors in patients with Rheumatoid Arthritis. The approximate time to complete the questionnaire is 5-10 minutes. To assure confidentiality, your responses will be coded and your name will not appear in any data analysis or research reports. Therefore, we assure you of as much confidentiality as legally possible. Your participation in this research study is voluntary. You do not need to answer all questions even though we would prefer that you do.

Your response will provide valuable information and is critical to the success of the research study. Please return the completed survey in the enclosed postage-paid business reply envelope.

If you have any questions or concerns, please do not hesitate to contact Khalid Kamal at (304) 293-1442 or Dr. Suresh Madhavan at (304) 293-1652 at the West Virginia University School of Pharmacy.

Thank you.

Sincerely,

Khalid Kamal, M.S.

Ph.D. Candidate
Suresh Madhavan, Ph.D.

Professor and Chairperson

Attachment 


\section{APPENDIX B: SECOND COVER LETTER}

November 15, 2004

Dear Physician:

About two weeks ago, we sent you a survey asking you about your current treatment practices and experiences using Tumor Necrosis Factor (TNF) inhibitors in patients with Rheumatoid Arthritis. If you have already completed and returned the survey, we thank you for your time and participation. If you have not completed the survey, we request you to kindly do so.

We understand that you are busy or may not have received the initial survey. However, your views are extremely important to us, and the information obtained from this research study would be very useful in determining common practices and issues associated with the use of TNF inhibitors. Therefore, we are again sending you this survey and would appreciate it if you will take a few minutes to complete the survey and return it in the postage-paid business reply envelope.

This research study is part of a doctoral (Ph.D.) dissertation project and is being undertaken and funded by the West Virginia University School of Pharmacy. To assure confidentiality, your responses will be coded and your name will not appear in any data analysis or research reports. Therefore, we assure you of as much confidentiality as legally possible. Your participation in this research study is voluntary. You do not need to answer all questions even though we would prefer that you do.

If you have any questions or concerns, please do not hesitate to contact Khalid Kamal at (304) 293-1442 or Dr. Suresh Madhavan at (304) 293-1652 at the West Virginia University School of Pharmacy.

Thank you.

Sincerely,

Khalid Kamal, M.S.

Ph.D. Candidate
Suresh Madhavan, Ph.D.

Professor and Chairperson

Attachment 
APPENDIX C: STUDY SURVEY 


\section{Current Treatment Practices using Tumor Necrosis Factor Inhibitors in Rheumatoid Arthritis}

INSTRUCTIONS: The first section of the questionnaire gathers prescribing information on specific Tumor Necrosis Factor (TNF) inhibitors [adalimumab $\left(\operatorname{Humira}^{\mathbb{B}}\right)$, etanercept $\left(\right.$ Enbrel $\left.^{\circledR}\right)$, and infliximab $\left(\right.$ Remicade $\left.^{\mathbb{B}}\right)$ ] for patients with Rheumatoid Arthritis (RA). Please answer the questions carefully. All responses will be kept confidential.

\section{Section A}

1. In what patient population do you normally use the following TNF inhibitor/s: adalimumab $\left(\right.$ Humira $\left.^{\circledR}\right)$, etanercept $\left(\right.$ Enbrel $\left.^{\circledR}\right)$, and infliximab (Remicade $\left.{ }^{\circledR}\right)$ : (Please check all that apply)

\section{TNF inhibitors}

Adalimumab (Humira ${ }^{\circledR}$ )

Etanercept $\left(\right.$ Enbrel $\left.^{\circledR}\right)$

Infliximab (Remicade ${ }^{\circledR}$ )

2. I do not use TNF inhibitors

\section{Newly Diagnosed}

\section{Patient Population}

Severe RA

3. In patients diagnosed with Rheumatoid Arthritis in whom you determine a TNF inhibitor is necessary, which TNF inhibitor are you most likely to prescribe? (Please rank in the order of preference with 1 being your first choice)

\section{TNF Inhibitor \\ Adalimumab (Humira $\left.{ }^{\circledR}\right)$ \\ Etanercept $\left(\right.$ Enbrel $\left.^{\mathbb{B}}\right)$ \\ Infliximab (Remicade ${ }^{\circledR}$ )}

Rank

4. In patients who have severe RA and who have failed to respond to methotrexate, please indicate how you would use a TNF inhibitor: (Please check all that apply)
$\neg$ TNF inhibitor alone
$\square$ TNF inhibitor and one other DMARD
$\square$ TNF inhibitor and 2 other DMARDs $\square$ No TNF inhibitor
$\neg$ Other treatment or approach (please specify)

5. a. In your practice, have you ever switched any patient from one TNF inhibitor to a different TNF inhibitor due to inadequate response or side effects?
$\square$ No
$\neg$ Yes

b. If yes, please indicate in general, your reason(s) for switching 
INSTRUCTIONS: The following questions are regarding the use of [adalimumab (Humira ${ }^{\circledR}$ ), etanercept $\left(\right.$ Enbrel $\left.^{\circledR}\right)$, and infliximab (Remicade $\left.{ }^{\circledR}\right)$ in your practice. Please answer the questions carefully. All responses will be kept confidential.

\section{Section B}

\section{The following questions are regarding the use of Adalimumab (Humira ${ }^{\circledR}$ )}

a. Please indicate the typical dose and frequency of dosing of adalimumab (Humira ${ }^{\circledR}$ ) that you use for patients with RA in your practice.

Dose for newly diagnosed RA

Dose for mild RA

Dose for moderate RA

Dose for severe RA

$\begin{array}{ll}\text { mg every } & \text { week } \\ \text { mg every_w } & \text { week } \\ \text { mg every } & \text { week } \\ \text { mg every } & \text { week }\end{array}$

b. Which laboratory tests do you routinely order for adalimumab $\left(\mathrm{Humira}^{\circledR}\right)$ and how frequently do you order these tests? (Please check all that apply)
$\underline{\text { Tests }}$
$\underline{\text { Tests ordered }}$
How frequently?

Antinuclear Antibody test (ANA)

Complete blood cell count (CBC)

Chest radiography

Creatinine

Purified protein derivative (PPD)

\begin{tabular}{|c|c|}
\hline Every & \\
\hline Every & \\
\hline Every & \\
\hline $\begin{array}{l}\text { Every } \\
\text { Every }\end{array}$ & \\
\hline
\end{tabular}

\section{The following questions are regarding the use of Etanercept (Enbrel $\left.{ }^{\circledR}\right)$}

a. Please indicate the typical dose and frequency of dosing of etanercept $\left(\right.$ Enbrel $\left.^{\circledR}\right)$ that you use for patients with RA in your practice.

Dose for newly diagnosed RA

Dose for mild RA

Dose for moderate RA

Dose for severe RA

$\begin{array}{ll}\text { mg every } & \text { week } \\ \text { mg every_week } & \text { week } \\ \text { mg every } & \text { week } \\ \text { mg every } & \text { week }\end{array}$

b. Which laboratory tests do you routinely order for etanercept $\left(\right.$ Enbrel $\left.^{\circledR}\right)$ and how frequently do you order these tests? (Please check all that apply)

Tests $\quad$ Tests ordered How frequently?

$\begin{array}{lll}\text { Antinuclear Antibody test (ANA) } & \square & \text { Every } \\ \text { Complete blood cell count (CBC) } & \square & \text { Every week(s) } \\ \text { Chest radiography } & \square & \text { Every week(s) } \\ \text { Creatinine } & \square & \text { Every week(s) } \\ \text { Purified protein derivative (PPD) } & \square & \text { Every week(s) }\end{array}$

\section{The following questions are regarding the use of Infliximab (Remicade ${ }^{\circledR}$ )}

a. Please indicate the typical dose and frequency of dosing of infliximab (Remicade ${ }^{\circledR}$ ) that you use for patients with RA in your practice.

Dose for newly diagnosed RA

Dose for mild RA

Dose for moderate RA

Dose for severe RA mg every

mg every

mg every

mg every week week week week 
b. Which laboratory tests do you routinely order for infliximab $\left(\right.$ Remicade $\left.^{\circledR}\right)$ and how frequently do you order these tests? (Please check all that apply)

Antinuclear Antibody test (ANA)

Complete blood cell count (CBC)

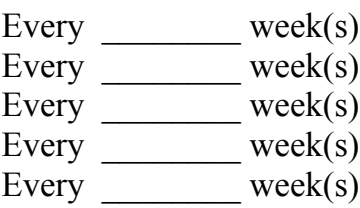

Chest radiography

Creatinine

Purified protein derivative (PPD)

Every week(s)

4. Please indicate the extent to which you feel each of the following factors are problematic to using specific TNF inhibitors in patients with Rheumatoid Arthritis. Circle the number on the continuum between 1 and $7(\mathbf{1}=$ no problem and $7=$ =major problem $)$ that best describes your opinion for each specific TNF inhibitor.

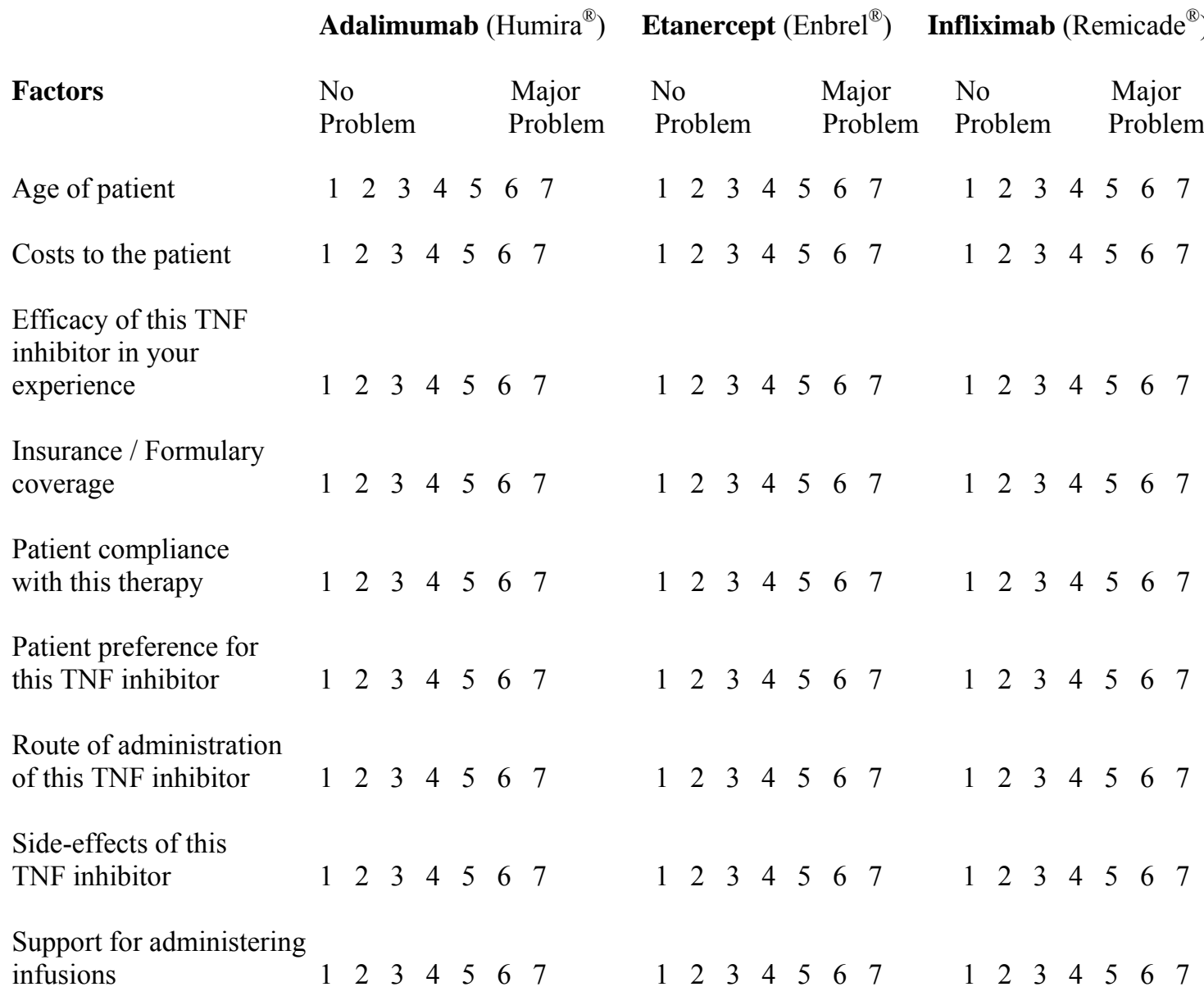


INSTRUCTIONS: This section of the questionnaire gathers information about you and your practice.

\section{Section C}

1. Age _ Years

2. Gender: $\quad \square$ Male $\square$ Female

3. Number of years in practice as a rheumatologist: Years

4. Your primary practice site is:

Hospital based $\square$ University-affiliated Hospital $\square$ Solo, Office-based $\square$ Group, office-based

Others, please specify

5. Average number of RA patients seen per week

6. Average number of prescriptions written per week for each drug:

$$
\begin{aligned}
& \text { Adalimumab (Humira }{ }^{\circledR} \text { ) } \\
& \text { Etanercept }\left(\text { Enbrel }^{\circledR}\right) \\
& \text { Infliximab (Remicade }{ }^{\circledR} \text { ) }
\end{aligned}
$$

7. Please indicate which of the following monitoring guidelines you follow for patients with RA on adalimumab $\left(\right.$ Humira $\left.^{\circledR}\right)$, etanercept $\left(\right.$ Enbrel $\left.^{\circledR}\right)$, or infliximab $\left(\right.$ Remicade $\left.^{\circledR}\right)$ ?

\section{ACR EULAR ACR \& EULAR}

None Other

(Please specify)

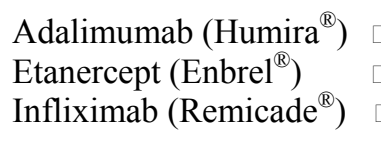

\section{COMMENTS}

If there is anything else that you would like to tell us, please use this space to provide your comments.

****THANK YOU FOR YOUR TIME AND ASSISTANCE!****

Please return the completed survey in the enclosed business reply envelope. 


\section{APPENDIX D: NON-RESPONSE SURVEY}

\section{Current Treatment Practices Using TNF Inhibitors in Rheumatoid Arthritis: Non-Response Survey}

Dear Physician:

During the past two months you may have received two or three mailings of a questionnaire asking you about your current treatment practices and experiences using Tumor Necrosis Factor (TNF) inhibitors in patients with Rheumatoid Arthritis. We realize that you have been very busy and have chosen not to answer the survey. Since your views are extremely important to us, we would like to know your reason for not responding to this survey and some key information for the research study.

This research study is part of a doctoral (Ph.D.) dissertation project and is being undertaken and funded by the West Virginia University School of Pharmacy. Your participation in this research study is voluntary. You do not need to answer all questions even though we would prefer that you do. Your responses will be coded, and your name will not appear in any data analysis or research reports. Therefore, we assure you of as much confidentiality as legally possible.

Please answer the few questions below and mail it to us in the postage-paid business reply envelope. If you have any questions or concerns, please do not hesitate to contact us at (304) 293-1442 or (304) 293-1652.

Sincerely,

Khalid Kamal, M.S. $\mathrm{Ph}$.D. Candidate
Suresh Madhavan, Ph.D. Professor and Chairperson

Q1. I did not respond to the survey because:

I did not receive it

Forgot the survey

I did not have enough time to complete it

The survey was misplaced

Survey was biased

Topic was irrelevant
I do not respond to mail surveys

The survey was too long

The survey was confusing

I am not interested in such studies

No incentive to complete it

Other reasons (Please

specify): 
Q2. In what patient population do you normally use the following TNF inhibitor/s:

adalimumab (Humira ${ }^{\circledR}$ ), etanercept $\left(\right.$ Enbrel $\left.^{\circledR}\right)$, and infliximab (Remicade $\left.{ }^{\circledR}\right)$ : (Please check all that apply)

\section{Patient Population}

TNF inhibitors Newly Diagnosed Mild RA Moderate RA Severe RA Adalimumab (Humira ${ }^{\circledR}$ )

Etanercept $\left(\right.$ Enbrel $\left.^{\circledR}\right)$

Infliximab $\left(\right.$ Remicade $\left.^{\circledR}\right)$

Q3. I do not use TNF inhibitors

Q4. Demographics:

1. Age __ Years

2. Gender: $\quad \square$ Male $\quad \square$ Female

3. Number of years in practice as a rheumatologist:

Years

4. Your primary practice site is:

$\square$ Hospital based $\quad \square$ University-affiliated Hospital $\quad \square$ Solo, Office-based

$\square$ Group, office-based $\quad \square$ Others, please specify

6. Average number of RA patients seen per week 


\section{CURRICULUM VITAE}

103 Ellen Lane

Phone: (304) 598-5670

Morgantown

Cellular: (304) 685-5984

WV 26505

Email:kkamal@hsc.wvu.edu

\section{PROFESSIONAL PROFILE}

Highly motivated candidate with diverse educational, work, research, and teaching experiences in the field of health economics and outcomes research

\section{Areas of Expertise}

- Decision modeling, epidemiology, pharmacoeconomics, survey research, and health services marketing

- Strong writing, research, and communications skills

- Strong background in computer applications and statistical skills

- Successful experiences in pharmaceutical sales and marketing

\section{ACADEMIC CREDENTIALS}

08/1999 - 05/2005

Doctor of Philosophy (Ph.D.) in Pharmaceutical Sciences

Major Area: Health Economics and Outcomes Research

Department of Pharmaceutical Systems \& Policy, West Virginia University

Dissertation: Assessing the cost-effectiveness of tumor necrosis factor inhibitors and prescribing practices of rheumatologists in patients with rheumatoid arthritis

Major Advisor: Suresh Madhavan, MBA, PhD

GPA: 3.71

08/1999 - 12/2000

Certificate in Health Care Administration, Department of Public Administration, West Virginia University

07/1994 - 06/1996

Masters in Pharmacology (MS), University of Bombay, India

06/1990 - 06/1994

Bachelor of Pharmacy (B. Pharm), University of Bombay, India

\section{WORK / RESEARCH EXPERIENCE}

06/2000 - 09/2004

CDC-funded Prevention Research Center, West Virginia University

Research Assistant

Developed evaluation measures, performed data analysis, assisted in writing grants and manuscripts, and devised marketing plans for Center-sponsored programs 
08/2003 - 06/2004

\section{Health Education Assessment Project in West Virginia}

Research assistant on a study funded by the West Virginia Department of Education. The project involved analyzing and reporting the first statewide assessment of health education. Developed analytical plan and assisted in preparation of report and publication of study results

05/2003 - 08/2003

\section{GlaxoSmithKline (Global Health Outcomes), RTP, NC}

Summer Intern

Selected Projects:

- Impact of treatment for allergic rhinitis on asthma-related health care utilization

- Developed conceptual framework, methodology, and data analysis plan using retrospective claims database

- Economic burden of illness among patients with allergic rhinitis and comorbid asthma

- Developed concept proposal and assisted in vendor evaluation

- Performed comprehensive evaluation of quality of life instruments, patient preference, and productivity measures

- Economic and humanistic outcomes in multiple sclerosis

- Developed a comprehensive report for economic and quality of life studies in support of product launch decision

07/2001 - 05/2002

Pharmacy \& Immunization Services: Pharmacists' Participation and Impact Research assistant on a study funded by the American Pharmacists Association (APhA) to assess pharmacists' involvement in immunization services. Assisted in development of survey questionnaire, data collection, analyses and publication of the study results

07/1996 - 06/1999

\section{Universal Medicare Ltd, Mumbai, India}

- Product Management

12/1997 - 06/1999

Successfully coordinated all aspects of product launch including annual marketing plans, situation analysis, competitive evaluation, sales planning, promotions, and pricing

- Area Sales Manager 12/1996 - 11/1997 Managed six sales representatives that consistently met or exceeded company sales goals

- Medical Sales Representative 07/1996 - 11/1996

Delivered effective sales presentations to physicians and successfully penetrated new markets 


\section{TEACHING EXPERIENCE}

08/1999 - 05/2000, 10/2004 - Present

Graduate Teaching Assistant, School of Pharmacy, West Virginia University

- Pharmacy as a Profession: Responsibilities included lecturing, grading, managing course materials, and facilitating student projects

- Pharmacy Care Lab and Patient Health Education: Responsibilities included conducting skills exercises, evaluating student verbal and non-verbal patient consultation skills, grading, and managing course materials

\section{RELEVANT COURSES COMPLETED}

Health Outcomes: Pharmacoeconomics, Outcomes and Quality of Life Assessment, Decision Analysis in Healthcare, Econometrics, Project Analysis and Evaluation, Health Behavior Theories

Research Methods: Epidemiology, Survey Research, Qualitative Methods

Statistical Methods: Data Management and Analyses, Multivariate Analysis, Regression Techniques

Health Administration: Healthcare Organizations and Operations, Health Systems, Managed Care

Marketing: Health Services Marketing, Consumer Behavior

COMPUTER SKILLS

Statistical packages: $\quad$ SPSS, SAS, STATA

Decision analysis software: DATA TreeAge, @ RISK, Precision Tree

Other: $\quad$ MS Office, Microsoft FrontPage, Microsoft Visio

\section{PUBLICATIONS}

Tompkins NO, Kamal KM, Chapman D. West Virginia Health Education Assessment Project. Journal of School Health 2005;75(6):193-198

Kamal KM, Miller LA. Psychosocial factors and asthma. (Letter). American Journal of Respiratory and Critical Care Medicine 2004;169(11):1253-54

Orsini L, Limpa-Amara S, Crown WH, Stanford RH, Kamal KM. Asthma hospitalization risk and costs for patients treated with fluticasone propionate versus montelukast. Annals of Asthma, Allergy and Immunology 2004;92:523-529

Dino GA, Kamal KM, Kalsekar ID, Fernandes AW, Horn KA. Stages of change and smoking cessation outcomes in adolescents. Addictive Behavior 2004;29(5):935-40

Kamal KM, Madhavan SS, Maine LL. Pharmacy and immunization services: Pharmacists' participation and impact. Journal of American Pharmaceutical Association 2003;43(4):470-82

Kamal KM, Madhavan SS, Amonkar MM. Determinants of adult influenza and pneumonia immunization rates. Journal of American Pharmaceutical Association 2003;43(3):403-11 
Kamal KM, Madhavan SS, Maine LL. Pharmacists and immunization services: Impact of American Pharmaceutical Association's (APhA) Immunization Training Certification Program. American Journal of Pharmaceutical Education 2003;67(4):article 124

Kamal KM, Madhavan SS, Maine LL. Pharmacists and immunization services: Impact of American Pharmaceutical Association's (APhA) Immunization Training Certification Program. (Abstract). Journal of American Pharmaceutical Association 2003;43(2):314

Kamal KM, Madhavan SM, Amonkar MM. Determinants of adult influenza and pneumonia immunization rates. (Abstract). Journal of American Pharmaceutical Association 2001; 41(2):320

Nair AM, Mungantiwar AA, Kamal KM, Saraf MN. Modulation of invitro anaphylaxis by furosemide. Indian Journal of Pharmacology 1997;29:182-186

Nair AM, Mungantiwar AA, Kamal KM, Saraf MN. Antiallergic potential of furosemide. Indian Journal of Experimental Biology 1997;35:466-469

Kamal KM, Madhavan SM, Hornsby JA, Miller LA, Scott V, Kavookjian J. Survey of current treatment practices of rheumatologists in prescribing tumor necrosis factor inhibitors in patients with rheumatoid arthritis (in preparation for Arthritis and Rheumatism)

Kamal KM, Miller LA, Madhavan SM, Kavookjian J. Alternative decision analysis modeling in the economic evaluation of tumor necrosis factor inhibitors in patients with rheumatoid arthritis (in preparation for Seminars in Arthritis and Rheumatism)

Tessaro I, Campbell MK, Kamal KM, Benedict S, Kelsey K, DeVellis B. Factors associated with breast and cervical cancer screening in a population of working women (in preparation)

\section{SELECTED SCIENTIFIC PRESENTATIONS}

Kamal KM, Miller LA, Madhavan SM, Kavookjian J. Alternative decision analysis modeling in the economic evaluation of tumor necrosis factor inhibitors in patients with rheumatoid arthritis. The annual meeting of the International Society for Pharmacoeconomics and Outcomes Research (ISPOR), Washington, D.C., May 2005

Kamal KM, Madhavan SM, Hornsby JA. Survey of current treatment practices of rheumatologists in prescribing tumor necrosis factor inhibitors in patients with rheumatoid arthritis. The annual meeting of the International Society for Pharmacoeconomics and Outcomes Research (ISPOR), Washington, D.C., May 2005

Kamal KM, Kalsekar I, Fernandes A, Horn K, Dino G. Stage of change and smoking cessation outcomes in adolescents. Presented at the annual meeting of the National Conference on Tobacco and Health, San Francisco, CA, November 2002 
Hassan M, Kamal KM, Mody R, and Amonkar M. Breast and cervical cancer screening among obese women in the United States. Presented at the annual meeting of American Public Health Association, Atlanta, GA, October 2001

Kamal KM, Madhavan SM, Amonkar MM. Predictors of influenza immunization rates in adults aged 50-64 years and 65 years and above. Presented at the annual meeting of the National Immunization Conference, Atlanta, GA, May-June 2001

\section{MANUSCRIPT REVIEWER}

Clinical Therapeutics

\section{HONORS/ AFFILIATIONS}

Treasurer of WVU-ISPOR student chapter (2002 - 2003)

Secretary of WVU-ISPOR student chapter (2001 - 2002)

Recipient of Graduate Research Award, School of Pharmacy, WVU (2001- 2002)

Recipient of All India Council of Technical Education Junior Research Fellowship (1994 - 1996)

Member of Rho Chi Honor Society

Member of International Society for Pharmacoeconomics \& Outcomes Research (ISPOR)

Member of American Pharmacists Association (APhA)

\section{REFERENCES}

Upon request 